\title{
DEEP ANISOTROPIC STRUCTURE UNDER THE CENTRAL VOLCANIC REGION, NEW ZEALAND
}

\author{
by \\ Sonja Melanie Greve
}

\author{
A thesis \\ submitted to the Victoria University of Wellington \\ in fulfilment of the \\ requirements for the degree of \\ Doctor of Philosophy \\ in Geophysics.
}

Institute of Geophysics

Victoria University of Wellington

Te Whare Wānanga o te Upoko o te Ika a Māui

Wellington

Aotearoa

Date of Defence

10. December 2008 


\section{Abstract}

Seismic anisotropy across the Hikurangi subduction zone measured from shear-wave splitting exhibits strong lateral changes over distances of about $250 \mathrm{~km}$. Teleseismic S-phases show trench-parallel fast polarisations with increasing delay times across the forearc and arc region. In the arc region, delay times reach up to $4.5 \mathrm{~s}$, one of the largest delay times measured in the world. Such large delay times suggest strong anisotropy or long travel paths through the anisotropic regions. Delay times decrease systematically in the backarc region. In contrast, local S-phases exhibit a distinct change from trench-parallel fast orientations in the forearc to trench-perpendicular in the backarc, with average delay times of $0.35 \mathrm{~s}$. In the far backarc, no apparent anisotropy is observed for teleseismic S-phases.

The three different anisotropic regions across the subduction zone are interpreted by distinct anisotropic domains at depth: 1) In the forearc region, the observed "average" anisotropy (about $4 \%$ ) is attributed to trench-parallel mantle flow below the slab with possible contributions from anisotropy in the slab. 2) In the arc region, high (up to $10 \%$ ) frequency dependent anisotropy in the mantle wedge, ascribed to melt, together with the sub-slab anisotropy add up to cause the observed high delay times. 3) In the far backarc region, the mantle wedge dynamic ends. The apparent isotropy must be caused by different dynamics, e.g. vertical mantle flow or small-scale convection, possibly induced by convective removal of thickened lithosphere.

The proposed hypothesis is tested using anisotropic wave propagation in two-dimensional finite difference models. Large-scale models of the subduction zone (hundreds of kilometres) incorporating the proposed anisotropic domains of the initial interpretation result in synthetic shear-wave splitting measurements that closely resemble all large-scale features of real data observations across the central North Island. The preferred model constrains the high (10\%) anisotropy to the mantle wedge down to about $100 \mathrm{~km}$ under the CVR, bound to the west by an isotropic region under the western North Island; the slab is isotropic and the subslab region has average (3.5\%) anisotropy, down to $300 \mathrm{~km}$. This model succeeds in reproducing the constant splitting parameters in the forearc region, the strong lateral changes across the CVR and the apparent isotropy in the far backarc region, as well as the backazimuthal variations.

The influence of melt on seismic anisotropy is examined with different small-scale (tens of kilometres) analytical modelling approaches calculating anisotropy due to melt occur- 
ring in inclusions, cracks or bands. Conclusions are kept conservative with the intention not to over-interpret the data due to model complexities. The models show that seismic anisotropy strongly depends on the scale of inclusions and wavelengths. Frequency dependent anisotropy for local and teleseismic shear-waves, e.g. for frequency ranges of $0.01-1 \mathrm{~Hz}$ can be observed for aligned inclusions on the order of tens of meters. To test the proposed frequency dependence in the recorded data, two different approaches are introduced. Delay times exhibit a general trend of $-3 \mathrm{~s} / \mathrm{Hz}$. A more detailed analysis is difficult due to the restricted frequency content of the data. Future studies with intermediate frequency waves (such as regional S-phases) are needed to further investigate the cause of the discrepancy between local and teleseismic shear-wave splitting.

An additional preliminary study of travel time residuals identifies a characteristic pattern across central North Island. Interpretation highlights the method as a valuable extension of the shear-wave splitting study and suggests a more detailed examination to be conducted in future. 


\section{Acknowledgements}

First of all, I am much obliged to my main advisor Martha Savage who encouraged me to apply for this $\mathrm{PhD}$ in the first place. Thanks you very much, Martha, for all your support, advice and guidance during these four years of my study.

Thanks to Euan Smith for helping me out with two chapters, and Tim Stern for reading and discussing an early version of chapter 5. Thanks you also to Ralph Wahrlich for the computer support.

Thanks to all my office mates over the year - Michelle Salmon, Mathieu Duclos, Bhaskara Rajasekhar Vadlamannati and Jessica Johnson, for bearing with me almost every single day, for chats and laughs and sharing all these good and bad times in the office.

Thanks to all my other colleges on the floor for being there for so many thing, especially Wanda Stratford for bike rides up at Makara, Sandra Bourguignon for being my gym buddy, Anya Seward for making me laugh, Adrian Benson for answering all these other annoying computer questions, Yannik Behr for German conversations, and Stacey Dravitzki for the good times at the summer school in Indonesia.

Thanks to my dad, for keeping me on track in countless telephone conversations; to my sisters Julia and Inga for all their emails, and to the rest of my family for always providing me a warm and relaxing home back in Germany.

Thanks you also to my friends in Germany and around the world for staying in touch through all the busy times. A special thank you to Mareike, for send the care parcel just when I needed it most.

Special thanks go to my friends outside the university, Naomi, Karen, Angelica, Gelya and for keeping me sane and to my flatmates Matthew and Rick, for friendship, for making me feel at home, for cooking great food, for crazy parties and for the dishwasher. Thank you, Erik, for standing all this through with me and for never giving up to make me smile.

I am also grateful for the help of so many people who supported my work, when once again, I got stuck: Mark Chapman, for providing the codes to calculate the frequency dependent anisotropy - just in the last minute; Ben Holtzman, for the preprint of the paper and the additional explanations about the modelling; David Okaya for discussions on FD modelling; Ian Billings from REF TEK and Alina Erias from Passcal for details about instrument 
responses; Mark Chadwick for help with GeoNet data; and Oliver Boyd for all the support for his code.

I would like to acknowledge GNS Science for supplying instruments and GeoNet for providing seismic data. Thanks also to Martin Reyners for providing the Leeds data set and Francis $\mathrm{Wu}$ for providing the data for WLZA. Many thanks go to all the field helpers for the WCNIPSE and CNIPSE deployment, especially Mark Henderson, Anya Seward, Erik Ewig, Martin Reyners, Graham Stuart, John Taber and Michelle Salmon. I also thank Katrina Marson-Pidgeon for modifying the automated splitting code for SKS phases and Mathieu Duclos for data contributions.

The main support for my studies came from a scholarship of the German Academic Exchange Service (DAAD) and the Victoria University PhD scholarship. Further funding, especially for the fieldwork, was provided by FRST, the Planet Earth Fund and the Lottery Board.

Most of the seismic processing in this study was done using the Seismic Analysing Code [SAC 2000; Tapley et al., 1990]. Further processing, especially for the frequency dependence analysis made use of the Matlab ${ }^{\circledR}$ software. The majority of the geographic maps is produced with the Generic Mapping Tools [GMT; Wessel \& Smith, 1995]. The thesis was typeset in $\mathrm{AT}_{\mathrm{E}} \mathrm{X}$. 


\section{Contents}

Abstract iii

Acknowledgments $\quad$ v

Table of Contents vii

List of Figures $\quad$ xi

List of Tables $\quad$ XV

Glossary xvii

1 Introduction 1

2 Background 5

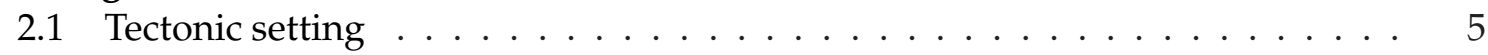

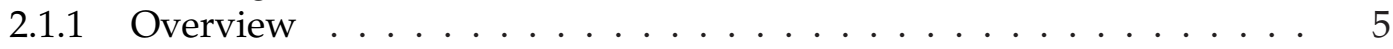

2.1.2 Geophysical signature of crust and upper mantle . . . . . . . . . . 9

2.2 The upper mantle and subduction zone: Conditions and materials . . . . . . . 13

2.2 .1 Temperature and pressure . . . . . . . . . . . . . . . 13

2.2.2 Subduction zone materials . . . . . . . . . . . . . . . . 13

2.2 .3 Water . . . . . . . . . . . . . . . . . . . . 15

2.2 .4 Melt . . . . . . . . . . . . . . . . . . 16

3 Seismic Anisotropy 19

3.1 Mathematical description of anisotropy - The elastic tensor . . . . . . . . . . . 19

3.2 Wave propagation in anisotropic media - Basic equations . . . . . . . . . . 22

3.3 Measuring seismic anisotropy . . . . . . . . . . . . . . . . 25

3.3.1 Elastic parameters . . . . . . . . . . . . . . . . . . 25

3.3.2 Shear-wave splitting parameters ..................... 26

3.4 Causes of seismic anisotropy in the Earth . . . . . . . . . . . . . . 28

3.4.1 Lattice-Preferred Orientation (LPO) . . . . . . . . . . . . . . . . 28

3.4 .2 Shape-Preferred Orientation (SPO) . . . . . . . . . . . . . . . . . . 31

3.5 Shear-wave splitting in subduction zones . . . . . . . . . . . . . . . . . 32

3.6 Shear-wave splitting in North Island . . . . . . . . . . . . . . . . 33

4 Data 37

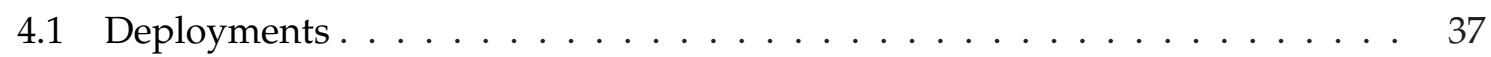

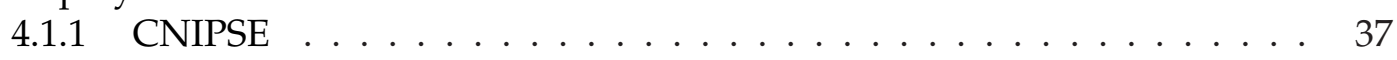

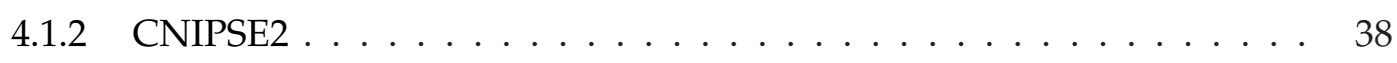

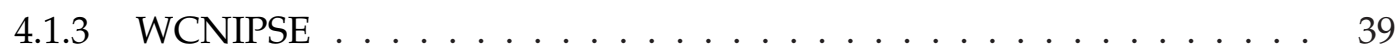


4.1 .4 NORD . . . . . . . . . . . . . . . . . . . . . . 40

4.1 .5 GeoNet. . . . . . . . . . . . . . . . . . . 40

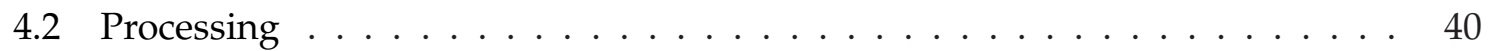

4.2.1 Preprocessing of passive seismic data . . . . . . . . . . . . 40

4.2 .2 Instrument correction . . . . . . . . . . . . . . . . . . 41

5 Shear-wave splitting across central North Island 47

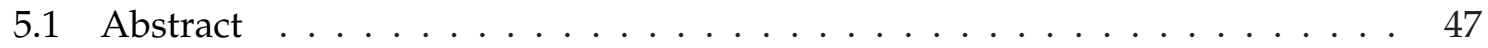

5.2 Introduction . . . . . . . . . . . . . . . . . . . 48

5.3 Method and data: Automated shear-wave splitting measurements . . . . . 52

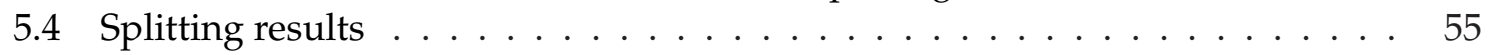

5.4 .1 SKS- and SKKS-phases . . . . . . . . . . . . . . . . . 55

5.4 .2 S- and ScS-phases . . . . . . . . . . . . . . . . . . . 56

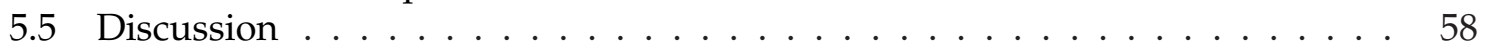

5.5 .1 Locating anisotropy . . . . . . . . . . . . . . . . . 59

5.5 .2 Causes for anisotropy $\ldots \ldots \ldots \ldots 62$

5.5 .3 Causes for apparent isotropy . . . . . . . . . . . . . . 66

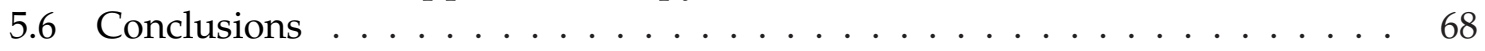

6 Modelling large-scale seismic anisotropy across central North Island 71

6.1 Two-layer modelling . . . . . . . . . . . . . . . . . 72

6.2 Finite difference modelling . . . . . . . . . . . . . . . . . . 73

6.2 .1 Theory . . . . . . . . . . . . . . . . . . 74

6.2 .2 Modelling the central North Island . . . . . . . . . . . . . . 77

6.3 Discussion and Conclusions . . . . . . . . . . . . . . . . . . . . 83

7 Modelling small-scale anisotropy $\quad 87$

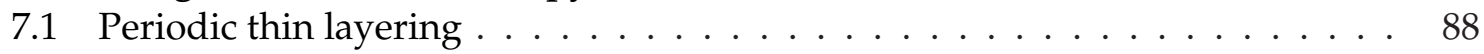

7.2 Oriented cracks and inclusions . . . . . . . . . . . . . . . . . 89

7.2 .1 Hudson theory . . . . . . . . . . . . . . . . . 90

7.2.2 Tandon and Weng theory . . . . . . . . . . . . . . . . . . . . 91

7.2 .3 Thomsen theory . . . . . . . . . . . . . . . . . 92

7.3 Chapman's frequency dependent model . . . . . . . . . . . . . . . 94

7.4 Frequency dependent fast polarisations . . . . . . . . . . . . . . . . 97

7.5 A combined model for the mantle . . . . . . . . . . . . . . . . . . . . 98

7.6 Analytical modelling results . . . . . . . . . . . . . . . . . . . . 99

7.6.1 Anisotropy due to oriented melt . . . . . . . . . . . . . . . . . 99

7.6.2 Frequency dependent anisotropy due to oriented melt . . . . . . . . 102

7.7 Discussion . . . . . . . . . . . . . . . . . . . . . . 104

$\begin{array}{lll}8 & \text { Evaluation of frequency dependent anisotropy } & 107\end{array}$

8.1 Frequency dependent shear-wave splitting . . . . . . . . . . . . . . . . . . 108

8.2 Wavelet analysis . . . . . . . . . . . . . . . . . . . . . 111

8.3 Discussion of frequency dependent analysis results . . . . . . . . . . . . . 114

8.4 Conclusions . . . . . . . . . . . . . . . . . . . . . . . . . . 119

9 Travel time residuals 121

9.1 Adaptive stacking method . . . . . . . . . . . . . . . . . . . . . . . 122

9.2 Data . . . . . . . . . . . . . . . . . . . . . . . 124

9.3 Travel time residuals along the CNIPSE line . . . . . . . . . . . . . . . . . . 125

9.4 Discussion . . . . . . . . . . . . . . . . . . . . . . . 126 
9.4.1 Isotropic velocity and structural changes . . . . . . . . . . . . . 126

$9.4 .2 \mathrm{~S} / \mathrm{P}$ ratio . . . . . . . . . . . . . . . . . . . . 130

9.4 .3 Anisotropic velocities . . . . . . . . . . . . . . . . . 132

9.5 Conclusion and Outlook . . . . . . . . . . . . . . . . . . . . 134

$\begin{array}{ll}10 \text { Conclusions } & 137\end{array}$

$\begin{array}{ll}\text { Appendices } & 141\end{array}$

$\begin{array}{ll}\text { A Elastic tensors } & 141\end{array}$

$\begin{array}{lll}\text { B Station details } & 143\end{array}$

$\begin{array}{ll}\text { C Processing details } & 147\end{array}$

C.1 ORION processing . . . . . . . . . . . . . . . . . . 147

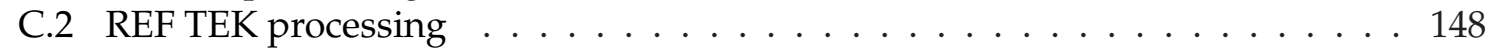

C.3 Instrument correction details $\ldots \ldots \ldots \ldots \ldots \ldots \ldots$

$\begin{array}{ll}\text { D Data } & 151\end{array}$

D.1 Event lists . . . . . . . . . . . . . . . . . . . . . . . . . 151

D.1.1 Event used for shear-wave splitting analysis . . . . . . . . . . . 151

D.1.2 Event used for travel time residuals analysis . . . . . . . . . . . . . . . 154

D.2 Spectra . . . . . . . . . . . . . . . . . . . . . . 154

$\begin{array}{lll}\text { E Shear-wave splitting results } & 157\end{array}$

E.1 Results from automated splitting code . . . . . . . . . . . . . . . 157

E.2 Shear-wave splitting analysis using SplitLab . . . . . . . . . . . . . 163

$\begin{array}{llr}\text { F Analytical small-scale modelling } & 169\end{array}$

F.1 Tandon and Weng theory - Terms . . . . . . . . . . . . . . . . . 169

F.2 Thomsen parameter . . . . . . . . . . . . . . . . . . . . 170

F.3 Modelling frequency dependent anisotropy . . . . . . . . . . . . . . . . 172

F.3.1 Testing the Matlab ${ }^{\circledR}$ code . . . . . . . . . . . . . . . . . . . . . 172

F.3.2 Frequency dependent elastic parameters . . . . . . . . . . . . . 173

G Two-layer modelling 177

G.1 Horizontal symmetry axis . . . . . . . . . . . . . . . . . . . . . 177

G.2 Dipping symmetry axis . . . . . . . . . . . . . . . . . . . . 183

$\begin{array}{ll}\text { H Travel time residuals } & 187\end{array}$

H.1 Travel time residuals for fast and slow components . . . . . . . . . . . . . 187

H.2 Travel time residuals due to crustal thickness variations . . . . . . . . . . . 188

$\begin{array}{lr}\text { References } & 189\end{array}$

Greve, 2008 ix 


\section{List of Figures}

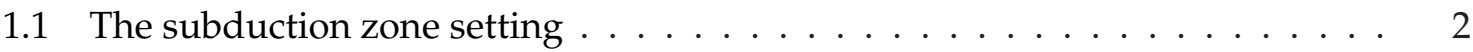

2.1 Tectonic setting of New Zealand . . . . . . . . . . . . . . . . . 6

2.2 Geological map of North Island . . . . . . . . . . . . . . . . . . . 8

2.3 Three dimensional perspective view of earthquakes under central North Island 9

2.4 Crustal and upper mantle structure from active source seismic . . . . . . . . . 10

2.5 Isostatic gravity map and anomalous upper mantle region under central North Island . . . . . . . . . . . . . . . . . . . . . . . . 11

2.6 Temperature and pressure conditions in a subduction zone . . . . . . . . . 13

3.1 Hierarchy of symmetry classes . . . . . . . . . . . . . . . . . 20

3.2 The most common anisotropic symmetries in the Earth . . . . . . . . . . . . 21

3.3 Direction dependent compressional and shear-wave velocities in an olivine

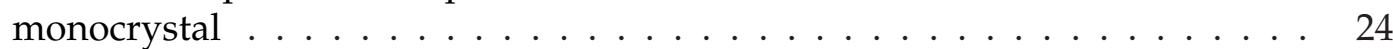

3.4 Seismic waves travelling through an anisotropic medium . . . . . . . . . . 25

3.5 Calculated North Island xenolith average seismic properties . . . . . . . . . . 26

3.6 Anisotropic regions of the Earth . . . . . . . . . . . . . . . . . 28

3.7 Lattice-preferred orientation . . . . . . . . . . . . . . . . . . . . . . . . . . . 29

3.8 Different types of olivine LPO . . . . . . . . . . . . . . . . . . . . 30

3.9 Shape-preferred orientation . . . . . . . . . . . . . . . . . . . 31

3.10 Summary of teleseismic shear-wave splitting in North Island . . . . . . . . . . 34

3.11 Local shear-wave splitting results across central North Island . . . . . . . . . 35

4.1 Station distribution across North Island . . . . . . . . . . . . . . . . . 38

4.2 Station set up for broadband recorder . . . . . . . . . . . . . . . 39

4.3 Amplitude response . . . . . . . . . . . . . . . . . . . . 42

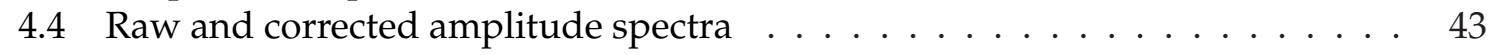

4.5 Comparison of cluster test recordings . . . . . . . . . . . . . . . . 44

5.1 Overview map . . . . . . . . . . . . . . . . . . . . . . . . 49

5.2 Map with splitting measurements . . . . . . . . . . . . . . . . 51

5.3 Three examples of automated splitting measurements . . . . . . . . . . . 53

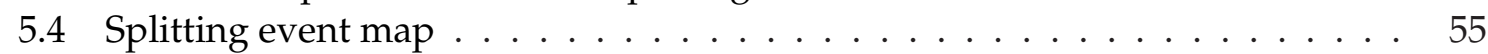

5.5 Record section . . . . . . . . . . . . . . . . . . . . . . . 57

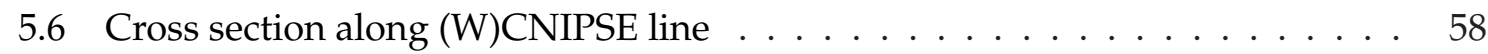

5.7 Frequency dependent shear-wave splitting . . . . . . . . . . . . . 65

5.8 Model for anisotropic regions across North Island . . . . . . . . . . . . . 68

6.1 Two approaches for two-layer modelling . . . . . . . . . . . . . . 72

6.2 Finite difference modelling method . . . . . . . . . . . . . . . 75 
6.3 Geometry of the Hikurangi subduction zone model space . . . . . . . . . . . 78

$6.42 \mathrm{D}$ geometry for finite difference modelling . . . . . . . . . . . . . . 79

6.5 Results for two inferior finite difference models . . . . . . . . . . . . . . . . . 81

6.6 Results for preferred finite difference model . . . . . . . . . . . . . . 82

7.1 PTL model for two media $\ldots \ldots \ldots \ldots \ldots$

7.2 Crack model . . . . . . . . . . . . . . . . . . . . . . . . . 89

7.3 Network fracture model . . . . . . . . . . . . . . . . . . . . . . 94

7.4 Combined model of melt-rich and depleted regions . . . . . . . . . . . . 98

7.5 Oriented melt pocket anisotropy ． . . . . . . . . . . . . . . . 100

7.6 Periodic thin layering of melt . . . . . . . . . . . . . . . . . . . . . . . 101

7.7 Anisotropy due to melt segregation - combined model . . . . . . . . . . . 102

7.8 Frequency dependent anisotropy due to melt inclusions . . . . . . . . . . . 103

7.9 Example for large-scale melt bands in the Oman ophiolite . . . . . . . . . . . 105

8.1 Period and frequency range covered by the analysis . . . . . . . . . . . . . 108

8.2 Results from frequency dependent shear-wave splitting . . . . . . . . . . . . 109

8.3 Frequency dependent splitting parameters for all "good" data with best linear fit . . . . . . . . . . . . . . . . . . . 110

8.4 Wavelets used for continuous wavelet transform . . . . . . . . . . . . . . 112

8.5 Estimating frequency dependent delay times with continuous wavelet transform . . . . . . . . . . . . . . . . . . . . . 113

8.6 Frequency dependence in shear-wave splitting delay times from continuous wavelet transform . . . . . . . . . . . . . . . . . . . . . . 115

8.6 Frequency dependence in shear-wave splitting delay times from continuous wavelet transform . . . . . . . . . . . . . . . . . . . 115

9.1 Station locations for travel time residual analysis . . . . . . . . . . . . . . 122

9.2 Example for adaptive stacking method . . . . . . . . . . . . . . . . 123

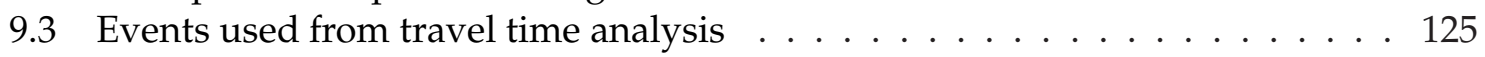

9.4 Travel time residuals for $\mathrm{P}$ - and S-waves . . . . . . . . . . . . . . 127

9.5 Map view of travel time residuals . . . . . . . . . . . . . . . . . . . 128

9.6 Cartoon of cross-section for central North Island . . . . . . . . . . . . . . 129

$9.7 \mathrm{~S} / \mathrm{P}$ delay time ratio . . . . . . . . . . . . . . . . . . . 130

9.8 Calculated fractional velocity changes . . . . . . . . . . . . . . . . . 131

9.9 Regional S/P delay time ratios . . . . . . . . . . . . . . . . . 132

D.1 Average spectrum for SKS-phases . . . . . . . . . . . . . . . . . . . 155

D.2 Average spectrum for S-phases . . . . . . . . . . . . . . . . . . . . . . 155

D.3 Average spectrum for ScS-phases . . . . . . . . . . . . . . . . 155

E.1 Example for a SplitLab measurement . . . . . . . . . . . . . . . . . . . . . 164

F.1 Frequency dependent shear-wave anisotropy for propagation direction of $70^{\circ}$

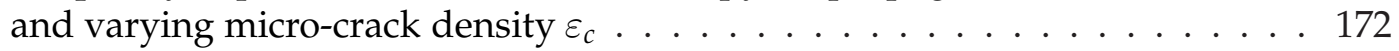

F.2 Frequency dependent quasi-shear velocity for propagation direction of $45^{\circ}$ for different crack fills . . . . . . . . . . . . . . . . . . 173

G.1 Anisotropic regions used for two-layer modelling . . . . . . . . . . . . . 178

G.2 Best-fit two-layer models for whole North Island . . . . . . . . . . . . . . . . 179

G.3 95\% confidence regions for best-fit model with horizontal symmetry axis for the full data set 
G.4 Best-fit two-layer models for three anisotropic regions in North Island . . . . 181

G.5 Results for two-layer modelling with dipping symmetry . . . . . . . . . . . . 184

G.6 95\% confidence region for the best-fit two-layer dipping symmetry axis model for the full data set . . . . . . . . . . . . . . . . . . . . 185

H.1 Travel time residuals for individual fast and slow polarisation . . . . . . . 187

H.2 Travel time residuals for average fast and slow polarisation . . . . . . . . 188 


\section{List of Tables}

3.1 Olivine fabrics, their abundance in rock samples and their dominant slip sys-

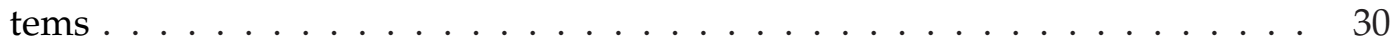

3.2 Shear-wave splitting in subduction zones . . . . . . . . . . . . 32

4.1 Event block structure . . . . . . . . . . . . . . . . . . . . . . 41

6.1 Parameters used for model setup . . . . . . . . . . . . . . . . . . 79

6.2 Finite difference models . . . . . . . . . . . . . . . . . . 80

7.1 Symmetries of ordered inclusions . . . . . . . . . . . . . . . . . . 990

7.2 Input parameters for Chapman model . . . . . . . . . . . . . . . 96

7.3 Input parameters for oriented melt pocket modelling . . . . . . . . . . . 99

7.4 Periodic thin layer modelling parameters . . . . . . . . . . . . . . . . 101

7.5 Input parameters for combined model . . . . . . . . . . . . . . . . . 102

7.6 Input parameters used to model melt inclusions in the mantle . . . . . . . . 103

8.1 Best linear fit for frequency dependent shear-wave splitting dataset . . . . . . 110

A.1 Full elastic tensors for some upper mantle materials . . . . . . . . . . . . . . 142

B.1 Deployment details . . . . . . . . . . . . . . . . . . . . . . 144

D.1 Events used for shear-wave splitting analysis . . . . . . . . . . . . . . . . 152

D.2 Events used for travel time residuals analysis . . . . . . . . . . . . . . . 154

E.1 Results of new and reprocessed single splitting measurements . . . . . . . . 158

E.2 Shear-wave splitting results from SplitLab . . . . . . . . . . . . . . . 165

G.0 Stations included in the different anisotropic regions used for anisotropic modelling . . . . . . . . . . . . . . . . . 178

G.1 Two-layer modelling results - horizontal symmetry . . . . . . . . . . . . . 182

G.2 Two-layer modelling results - dipping symmetry . . . . . . . . . . . . . 185 


\section{Glossary}

Adiabatic No heat exchange with surrounding environment, no conduction, e.g. convecting asthenosphere.

AK135 Global velocity model [Kennett et al., 1995], designed to provide a good fit to a wide variety of seismic phases and in particularly to improve the fit for $\mathrm{S}$ phases compared to the iasp91 model.

Anisotropy Material properties vary with azimuth/direction in which the measure was made, results from ordered heterogeneities smaller than the seismic wavelength.

Arc volcanic front The position of the arc volcanoes nearest the trench [Bebout, 2007].

Asthenosphere Region beneath lithosphere with temperatures above $1100^{\circ}$. Plastic deformation (flow) permits isostatic compensation and slow convection currents.

Backazimuth The angle measured between north and the direction to the source.

Birefringence Another word for shear-wave splitting meaning "double refraction", more commonly used in optics. The phenomenon by which one component of a transverse wave travels through an anisotropic medium faster than the orthogonal component. Birefringent material is a homogeneous solid that causes two waves of different polarisation to travel at different speeds in the same direction [Winterstein, 1990].

Diffusion creep A process in rock formation in fine-grained rocks under low stress conditions or high temperatures, which does not form LPO. The deformed material is isotropic. It is considered important below $250 \mathrm{~km}$ depth [e.g. Nicolas, 1984; Karato \& $\mathrm{Wu}, 1993]$.

Dislocation creep Main mechanism of deformation in upper mantle, caused by the motion of crystalline dislocations within grains, which results in preferred mineral orientation [Nicolas, 1984]. Displacement of intracrystalline dislocations within minerals with large grain size under high stress conditions or low temperatures (a third of the melting point). Due to the resulting intracrystalline strain the dominant slip system reorients parallel to the imposed shear. As a result of the reorganisation within the crystal, new grain boundaries can occur and new grains can form, called dynamic recrystallisation.

Dynamic recrystallisation occurs at higher strain and temperatures than dislocation creep, e.g. convective flow in the mantle [Kaminski \& Ribe, 2001]. Can occur as growth of pre-existing grains or formation of new grain boundaries (nucleation).

Extrinsic anisotropy Occurrence of anisotropy depends on the seismic wavelength used to measure the anisotropy (SPO, OMP). 
Fluid Disordered, non-crystalline phase consisting of particles in motion and possessing unspecified composition, commonly rich in molecules such as $\mathrm{H}_{2} \mathrm{O}$ and $\mathrm{CO}_{2}$ and containing dissolved major and trace elements [Bebout, 2007].

Harzburgite Depleted mantle rock, mainly consisting of olivine and orthopyroxene.

Heterogeneous Material properties such as velocity, porosity or density vary with position.

Hexagonal symmetry Symmetry with a single axis of six fold rotation. The seismic properties of hexagonally symmetric systems are identical to those of cylindrical symmetry, in which properties in the plane perpendicular to the symmetry axis are independent of direction [Babuška \& Cara, 1991].

Intrinsic anisotropy Anisotropy caused by small-scale, microscopic inhomogeneities, independent on the wavelength (LPO) [Best et al., 2007].

Lx-norm Measure of misfit [Davies, 1986] often used to minimise expressions like:

$$
\epsilon=\sum_{i=1}^{n}\left[\left|t_{i}-t_{i}^{p}\right|\right]^{x}
$$

The L2-norm $(x=2)$ or least square can tend to give too much weight on outliers, if the misfit is not caused by random nose. In this case, an L1-norm can be applied. However, as it uses the absolute difference of the values, this can cause further problems.

Lithosphere Crust and cold top of mantle, which deforms elastically (rigid). Oceanic lithosphere thickens with age up to $100 \mathrm{~km}$. Continental lithosphere is slightly thicker [Stein \& Wysession, 2003].

LPO Lattice-preferred orientation, alignment of crystals in a mineral in a preferred orientation.

Mantle Solid part of the Earth from base of the crust $(10-30 \mathrm{~km})$ to the core $(2900 \mathrm{~km})$. The upper mantle, down to $400 \mathrm{~km}$ is made up of peridotite. In the lower mantle peridotite transforms into denser high pressure phase.

Melt segregation Separation of melt from mantle source. Compaction is the differentiation of the crystalline matrix from the melt. Melt segregation occurs because of differences in density of partial molten basalt and crystalline matrix.

Oceanic lithosphere Tectonic plate typically about $50-100 \mathrm{~km}$ thick, consisting mainly of mafic crust (6-7 km thick) and ultramafic mantle [Bebout, 2007].

OMP Oriented melt pockets, alignment of melt inclusions in a preferred orientation.

Ophiolite Pieces of oceanic plate that have been thrust (obducted) onto the edge of continental plates [Turcotte \& Schubert, 2002]. Ophiolites have been found in Cyprus, New Guinea, Newfoundland, California, and Oman. On-land exposure of ancient oceanic crust, sometimes metamorphosed before emplacement [Bebout, 2007].

P-wave Compressional waves. Waves that vibrate, i.e. are polarised parallel to the travel (propagation) direction.

Peridotite Main upper mantle rock, consisting mainly of olivine. Compositions can include spinel alone (dunite), additional orthopyroxene (harzburgite) or orthopyroxene and clinopyroxene (lherzolite). 
PTL Periodic thin layering, layering of materials with different properties with layer thickness smaller than the seismic wavelength.

Pure shear Principal axis of stress and strain coincide.

Radial anisotropy Hexagonal anisotropy with a vertical symmetry axis.

S-wave Shear waves. Waves that vibrate, i.e. are polarised perpendicular to the travel (propagation) direction.

SH wave Pure shear-wave, horizontally polarised shear-waves.

SV wave Vertically polarised shear-waves.

Simple shear Principal axes of strain rotated with respect to the flow plane/direction with progressive deformation. Orientation of principal stress is $45^{\circ}$ with respect to the flow plane/direction.

Solidus Temperature and pressure conditions at which material undergoes partial melting.

Sonic band Frequencies between $10^{3}$ and $10^{4} \mathrm{~Hz}$, audible to the human ear.

SPO Shape-preferred orientation, alignment of heterogeneities, such as cracks or inclusions in a preferred orientation.

Squirt flow Dynamic of a fluid under the influence of an acoustic wave.

Squirt flow frequency A characteristic rock property, which depends on the details of the crack-pore microgeometry and the properties of the fluid. It is proportional to the permeability and inversely proportional to the viscosity of the fluid [Mavko, 1980]. Usually between the sonic and ultrasonic bands for brine-filled rocks [Thomsen, 1995]. At frequencies lower than the squirt flow frequency, there is enough time between each peak of the wave amplitude for the fluid pressure to equilibrate between cracks and pores. For higher frequencies, the elastic moduli will be stiffer and no flow is induced.

Subducting slab Generally an oceanic tectonic plate that sinks below an overriding continental or oceanic plate [Bebout, 2007].

Symmetry axis The axis around which a material can be rotated without changing the description of the material's properties.

Trench Contact line in subduction setting where downgoing plate meets overriding mantle.

Ultrasonic band Frequencies between $10^{5}$ and $10^{7} \mathrm{~Hz}$.

Volatiles Compounds that form low-viscosity fluids when released from a rock or magma [Bebout, 2007].

Xenolith Solid mantle rocks entrained in erupting magmas, carried up to the surface by volcanic flows [Turcotte \& Schubert, 2002]. 


\section{Chapter 1}

\section{Introduction}

Subduction zones are convergent plate margins, where dense oceanic lithosphere descends under overriding oceanic or continental lithosphere (figure 1.1). The subduction process evokes major implications for the Earth. Essentially, the sinking of the slab provides the central driving force for plate tectonics [Davies, 1999]. Moreover, the intrusion of the cold lithosphere, water and other incompatible materials into the mantle initiates numerous reactions in the "subduction factory". The products of the subduction factory, such as melt and continental crust, generate two well-known surface expressions of subduction zones - volcanoes and islands arcs. Volcanic eruptions, earthquakes caused by the downgoing plate, and tsunamis evoked by them are the hazards that accompany subduction [Uyeda, 1982].

While the negative buoyancy of the slab is established as the main cause of the dynamic nature of subduction zones [Conrad \& Lithgow-Bertelloni, 2002], details of the dynamics in the different subduction zone regions are still poorly understood. In contrast to the generally accepted theory that mantle flow is parallel to plate motion [Hager \& $\mathrm{O}^{\prime}$ Connell, 1981], more recent experimental models [Buttles \& Olson, 1998; Funiciello et al., 2006] indicate various different pattern in subduction settings (figure 1.1). Mantle wedge dynamics are often described by the cornerflow model (figure 1.1, option (1)), caused by viscous slab-wedge coupling [Peacock \& Wang, 1999]. Contrasting models suggest trench-parallel flow (figure 1.1, option (2)) in the wedge and below the subducting lithosphere induced by discontinuation of the slab [Peyton et al., 2001] or slab roll-back [Russo \& Silver, 1994]. But many more factors affect subduction dynamics; they include slab geometry and age [Kincaid \& Sacks, 1997; Billen, 2008], subduction and trench migration speed [Doglioni et al., 2007; Long et al., 2008], nature of the overriding plate, rheology of the wedge [King, 2001] and foundering of lithosphere [Behn et al., 2007]. Their influence enables more complex flow patterns (figure 1.1, option (3)). Even though the dynamic consequences of most of these factors are well-established, mainly due to advances in dynamical modelling [Gerya et al., 2004; Billen, 2008], the exact configurations between different subduction zones can vary broadly and are still enigmatic. 


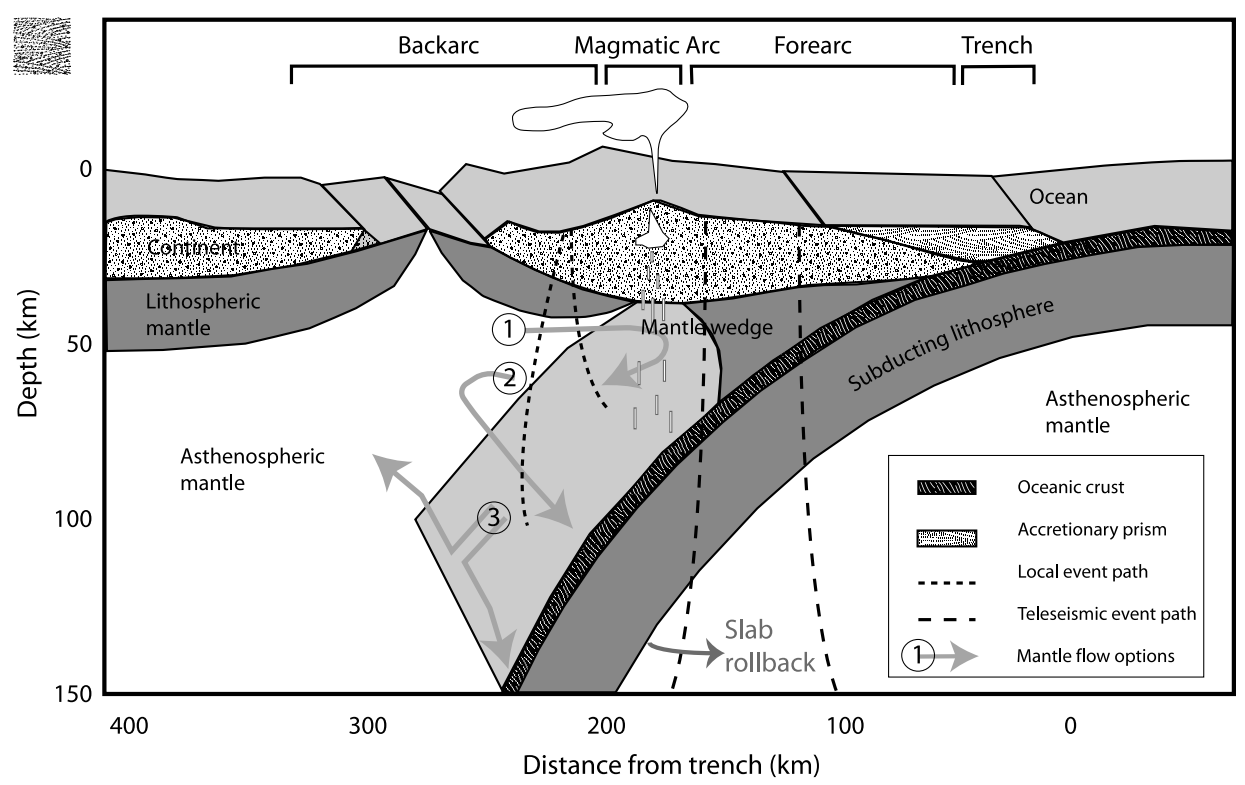

FIGURE 1.1 The subduction zone setting. Mantle flow options are: (1) Corner flow, (2) toroidal flow around the edges and (3) more complex, locally changing flow. For details see text.

Surface expressions can only provide limited insights into dynamic deformation processes in the subduction zone, as surface geology does not necessarily reflect dynamic processes at depth [Moores \& Twiss, 1995]. Moreover, the major part of most subduction zones is situated below sea level, hindering investigations. Seismic anisotropy provides the means to examine the structural geology of the lithosphere and flow induced dynamic deformation patterns down in the deep Earth [Silver et al., 1999; Park \& Levin, 2002]. The study of seismic anisotropy is based on the fact that deformation (due to stress or strain) creates preferred orientations, either of heterogeneities or of crystal minerals, in materials [Crampin \& Chastin, 2003; Silver, 1996; Savage, 1999]. This causes waves to travel faster in one direction than another.

For the geological and tectonical interpretation of seismic anisotropy it is important to understand the relationship between deformation and material texture, as well as the relation between rock texture and seismic velocity (chapter 3 ). The main knowledge is derived from naturally deformed mantle rock samples [Ismaï \& Mainprice, 1998; Mainprice, 2007], active deformation experiments [Karato et al., 2008] and a rapidly increasing number of numerical simulations. While seismic anisotropy has long been used as a direct measure for mantle flow directions [Ribe, 1989], it now becomes apparent that the interpretation is more complex. New modelling approaches relate density-driven convective mantle flow to observed anisotropy [Becker et al., 2003; Gaboret et al., 2003; Conrad et al., 2007]. But these global models only work well far from subduction zones, likely because flow patterns are more complicated in subduction zones, as explained above, and deformation is higher [Turcotte \& Schubert, 2002]. Other reasons could be due to the influence of water [Karato, 2003] and melt [Kendall, 1994; Holtzman et al., 2003b].

The presence of melt in the mantle wedge is established by the volcanic surface expres- 
sion. But its dynamics, distribution, and influence in the subduction zone are still vaguely determined. Current research investigates where melts forms in the mantle wedge, which form it occurs in and how it is transported to the volcanic front (section 2.2.4). To do so, partially molten samples can be created and observed under laboratory conditions [Holtzman et al., 2003b; Takei, 2001, 2005]. Still, measuring elastic behaviour at elevated temperaturepressure conditions is a major challenge. Experiments to determine velocities at seismic frequencies and in the presence of melt are difficult and is mainly limited to isotropic aggregates [e.g. Zimmerman et al., 1999; Jackson et al., 2002]. Thus, models assuming idealised shaped melt inclusions have been applied [Kendall, 1994; Mainprice, 1997]. Lab and modelling results establish a strong influence of melt on seismic properties. Additionally, in subduction zones the presence of water or other volatiles dramatically enhances not only melt production, but also alters the observed anisotropy [Jung \& Karato, 2001b; Karato, 2003].

The growing number of anisotropy studies in subduction zones highlights a large variety of results and interpretations [e.g. Wiens \& Smith, 2003] (cf. section 3.5). This confirms that every subduction setting is unique and has to be assessed individually. Due to the various factors influencing anisotropy in subduction zones, different research disciplines, including experimental research on mantle rheology [Karato et al., 2008] and advanced computational modelling [Levin et al., 2006] need to be combined. Moreover, for a complete picture the consideration of different scale-length heterogeneities becomes increasingly important [Helbig \& Thomsen, 2005; Tommasi et al., 2006; Holtzman \& Kendall, 2008].

New Zealand provides a great opportunity to study plate boundary deformation processes, due to its location on continental mass above the plate boundary (section 2.1). It is also one of the few places on Earth where the spreading backarc region is located on land [Hatherton, 1970]. The outstanding characteristic of the backarc setting is accentuated as it undergoes active continental rifting [Stern, 1985]. The special setting of New Zealand's North Island is accompanied by elevated heat output [Pandey, 1981] and an extremely high eruption rate in the extending Central Volcanic Region, making it one of the hottest and most productive magma generating regions on Earth [Wilson et al., 1995]. A series of recent studies highlights the significant role of melt on geophysical properties, such as velocities and attenuation, across upper North Island [Stratford \& Stern, 2004, 2006; Salmon et al., 2005; Stern et al., 2006; Seward et al., 2008].

Several shear-wave splitting studies have been conducted in North Island, but mainly cover the lower North Island (section 3.6). The predominantly trench-parallel anisotropy indicates a coherent deformation throughout the crust and lithosphere accompanied by trench-parallel astenospheric flow [e.g. Gledhill \& Gubbins, 1996; Marson-Pidgeon et al., 1999]. However, recent local shear-wave splitting studies indicate a dramatic change towards trench-perpendicular anisotropy further north in the volcanic region [Audoine et al., 2004; Morley et al., 2006]. But teleseismic measurements across the arc region, especially in the backarc, are sparse. 
This opens the question of the relationships between anisotropy, subduction zone dynamics and the presence of melt in the Hikurangi subduction zones. Thus, this study is devoted to provide insights into some aspects of these interrelations. In particular, the following points are addressed:

- Do shear-wave splitting parameters from teleseismic phases change across the arc system? (Chapter $\left.5^{1}\right)$

- How is the observed anisotropy distributed across central North Island? (Chapter 6)

- How does melt in the mantle wedge influence anisotropy? (Chapter 7)

- Does the measuring frequency affect the observed anisotropy? (Chapter 8)

An additional chapter (chapter 9) introduces some preliminary work on travel time residuals. This work is not finalised, but provides detailed processing and interpretation approaches that are discussed based on a preliminary data set.

${ }^{1}$ accepted for publication in Tectonophysics [Greve et al., 2008] 


\section{Chapter 2}

\section{Background}

\subsection{Tectonic setting}

\subsubsection{Overview}

New Zealand is situated on the plate boundary between the Pacific and the Indo-Australian Plate (figure 2.1). The Pacific plate is the largest and most active tectonic plate on Earth [Montagner, 2002], with its plate margin known as the "Ring of Fire". The New Zealand land mass is divided into two main islands, South and North Island. The plate boundary character changes throughout New Zealand, with a transpressive fault linking two opposite oblique subduction zones. At the southern tip of South Island, the Australian plate subducts under the Pacific plate along the Puysegur trench at a rate of $38 \mathrm{~mm} / \mathrm{a}$ [Demets et al., 1990]. In central South Island, plate motion is accommodated by continental collision and dextral strike-slip motion along the Alpine fault [Walcott, 1998]. The Marlborough fault system is the transition zone from the transpressive strike-slip system into another subduction zone [Little \& Jones, 1998]. On North Island, the subduction switches polarity with the oceanic crust of the Pacific plate subducting under the continental crust of the Indo-Australian plate. Plate motion of the subduction is oblique ( $50^{\circ}$ to the trench) with rates of $48 \mathrm{~mm} / \mathrm{a}$ in the north and about $42 \mathrm{~mm} / \mathrm{a}$ [Demets et al., 1990], with increased obliquity, in the south. The present subduction of an ancient volcanic plateau, the Hikurangi plateau, under North Island along the Hikurangi trench [Mortimer \& Parkinson, 1996] increases the buoyancy of the Pacific plate and therefore inhibits subduction. Subduction at the Hikurangi margin started about 30-24 Ma ago [Walcott, 1987; King, 2000], causing compression and tectonic shortening in the overriding Australian plate, progressing southward with time. In Early Pliocene (4-5 Ma) compression switched to extension [King, 2000]. Plate reconstructions require a rapid rotation of the Hikurangi margin relative to the Australian plate of about $6^{\circ}$ per Ma, for the last $5 \mathrm{Ma}$ [Walcott, 1987]. Recently, the presence of a new microplate has been inferred to argue for an earlier onset of the change in rotation rate, probably as early as 20 Ma ago [Cande \& Stock, 2004]. 


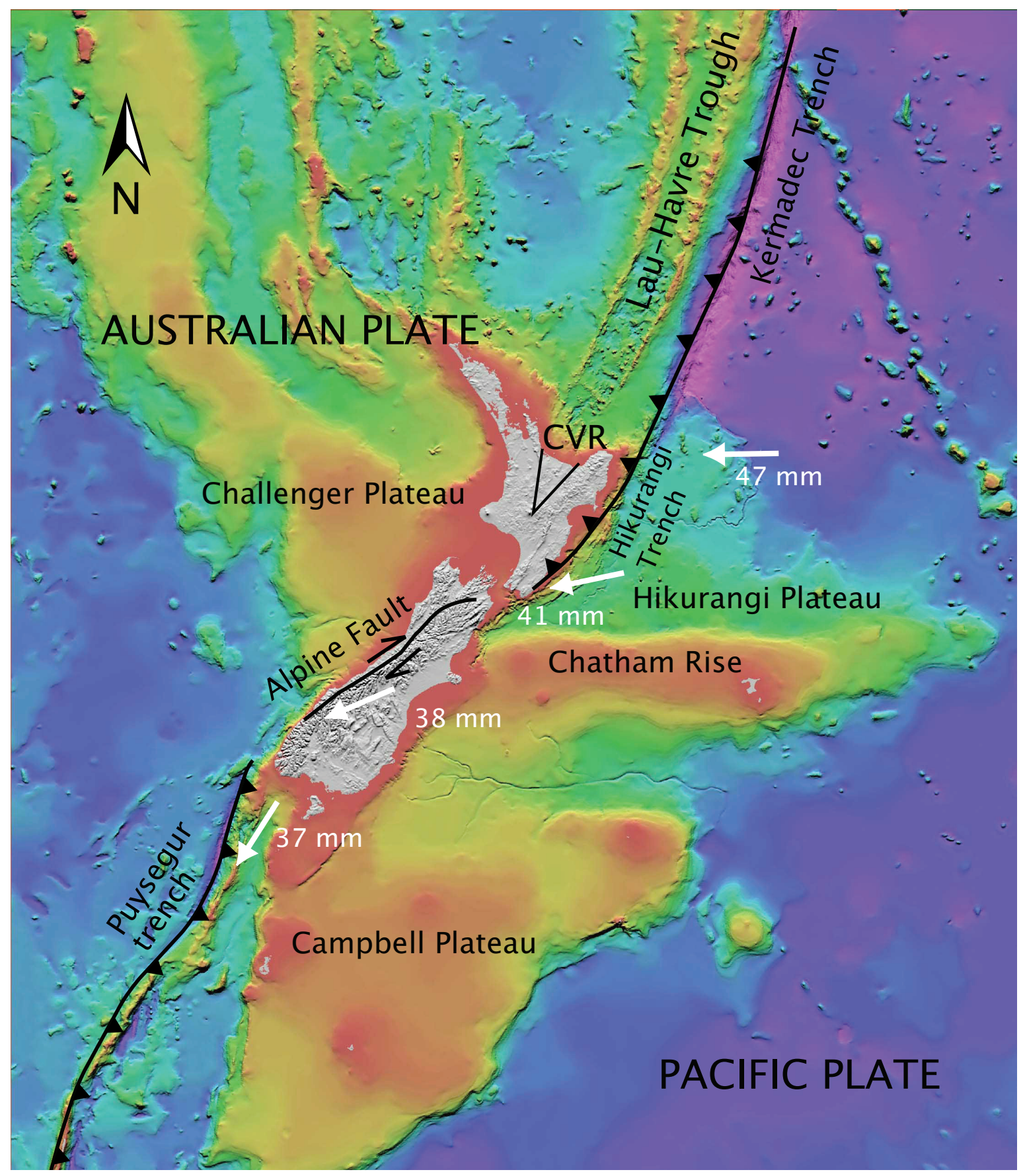

FIGURE 2.1 Tectonic setting of New Zealand, bathymetry from NIWA. White arrows indicate the direction of the Pacific Plate motion relative to the Australian plate. Note the change from oceanic backarc rifting in the Lau-Havre trough to continental rifting in the Central Volcanic Region (CVR), the backarc of the Hikurangi subduction. 
Even though the Hikurangi subduction zone forms the southernmost extension of the relatively simple Tonga-Kermadec system [Smith \& Price, 2006] (figure 2.1), it displays a more complex, three-dimensional character, especially in the backarc region. Unusual features of the Hikurangi margin include a shallow trench, a gentle dip of the subducted plate and a forearc and backarc setting above sea level [Smith et al., 1989].

The tectonic regime of the Hikurangi subduction zone is described as extensional in the north (Mariana-type subduction) and compressional (Chilean-type subduction) in the south [Stern et al., 2006]. Margin-parallel motion is taken up by faults in the overlying Australian plate; margin-normal motion takes place on the plate interface [Webb \& Anderson, 1998]. The change from transpressional to extensional deformation is also obvious in the thinning of the crust from $35 \mathrm{~km}$ in the south to $20 \mathrm{~km}$ in the north [Nicol et al., 2007] and a change in sediment thickness along the trench from more than $4 \mathrm{~km}$ in the south and central section to less than $1.5 \mathrm{~km}$ in the extreme north, where the Hikurangi plateau passes into more "normal" oceanic crust [Smith et al., 1989]. These anomalous features of the Hikurangi subduction zone might cause a change in plate coupling from south to north. The plate interface in the southern part of the Hikurangi margin is inferred to be currently locked, whereas the northern part is considered as stable riding, due to weaker coupling [Reyners et al., 1997; Wallace et al., 2004]

Southern central North Island is defined by high compression occurring within the currently uplifting axial ranges [Walcott, 1987] (figure 2.2). This part of North Island has undergone the highest amount of shortening, about $3-8 \mathrm{~mm} / \mathrm{a}$ (Post-Oligocene) [Nicol et al., 2007]. No volcanism is apparent in the southern part of the Hikurangi subduction zone, south of latitude $39^{\circ}$ [Funnell et al., 1996].

The forearc region on eastern central North Island is currently subject to uplift [Davy \& Wood, 1994], caused by the high buoyancy of the thick oceanic crust (12-15 km) of the oceanic Hikurangi Plateau [Mortimer \& Parkinson, 1996; Reyners et al., 2006]). Another implication of the subduction of dense, old oceanic lithosphere is relatively low stress along the plate boundary, giving rise to the rollback of the subducting slab [Stern, 1987; Smith et al., 1989]. Much of the strike-slip motion on the Hikurangi margin is accommodated on the North Island Fault System (NIFS) or North Island Dextral Fault Belt [Nicol et al., 2007] (figure 2.2). While forearc basin sediments dominate the geology of the east coast basin, greywacke-cored mountains build the North Island Faults System [Walcott, 1987].

The central North Island extends across the arc region of the subduction zone, including forearc, magmatic arc and backarc. Northwest-southeast extension [Cole, 1990; Darby \& Meertens, 1995] (starting in Early Pliocene) of 5-15 mm/a [Wallace et al., 2004] has formed a wedge-shaped region of thinned continental crust (15-18 km) [Stratford \& Stern, 2004]) called the Central Volcanic Region (CVR). The fan-like opening migrates southeast, possibly caused by the eastwards rollback of the Pacific plate [Walcott, 1987]. Extension is slowest at the southern tip of the CVR, defined by the southernmost volcano, Mt. Ruapehu, and increases to the north [Wallace et al., 2004]. The CVR marks the southern end of the Lau-Havre 


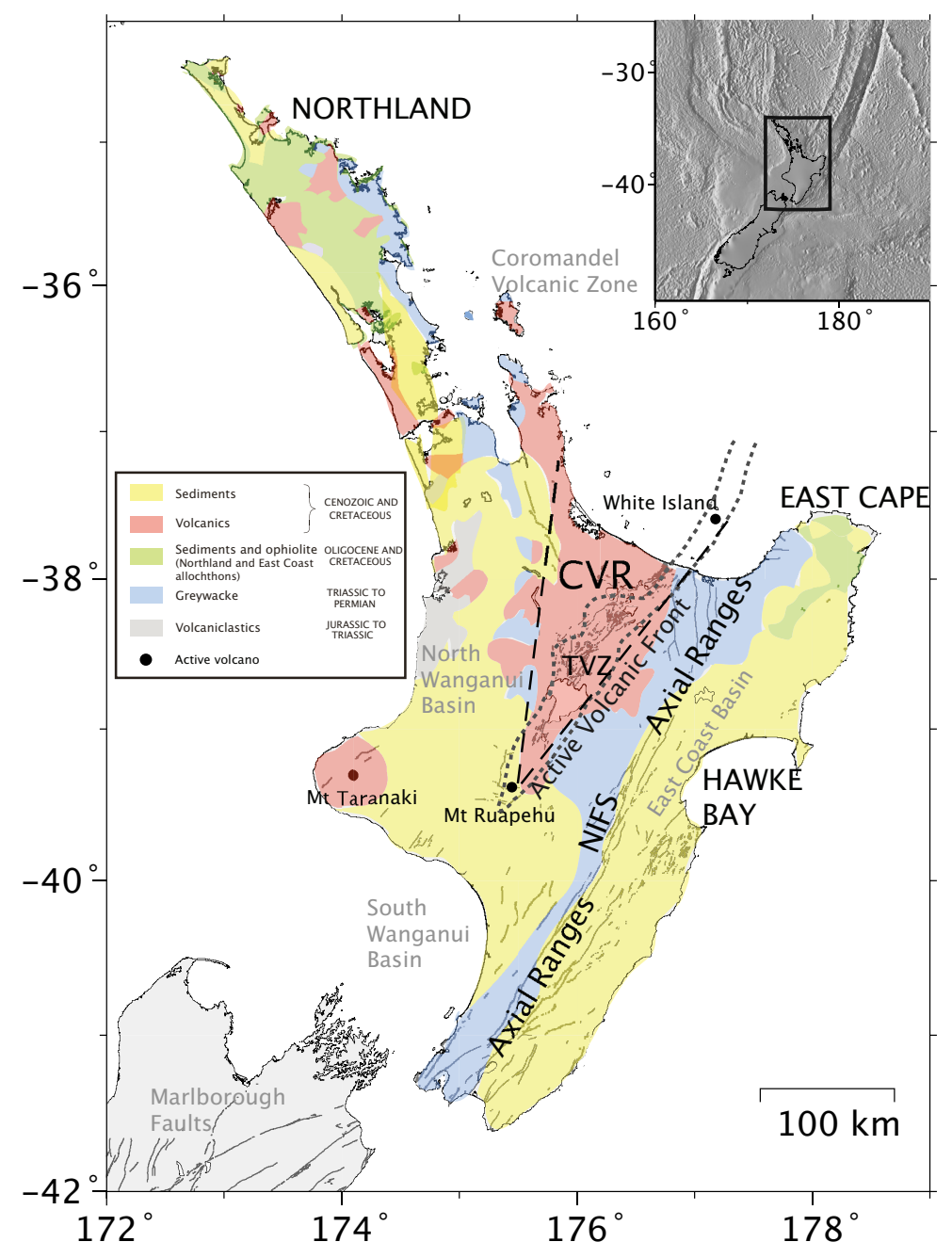

FIGURE 2.2 Geological map of North Island, based on map "New Zealand geology", IGNS [Forsyth et al., 1972].

trough and is therefore a region of transition between oceanic and continental rifting. Due to its relation to the subduction zone, the subsided region is often referred to as a "backarc basin" [Cole, 1990]. However, strictly speaking, this term refers to oceanic and not continental crust and therefore other terms, such as "marginal basin" and "rifted arc" [Wilson et al., 1995], have been used. The basin fill of the CVR consists mainly of volcanic deposits, reaching at least 2-3 km depth [Stern \& Davey, 1987] (figure 2.2).

Presently active silicic (andesitic and rhyolitic) volcanism takes place in the young Taupo Volcanic Zone (TVZ, 0.34 Ma), along the eastern half of the CVR [Cole, 1990]. The approximately $200 \mathrm{~km}$ long and $60 \mathrm{~km}$ wide TVZ is considered as the volcanic arc of the subduction zone, trending about $30^{\circ}$ north, with two active volcanoes - Mt. Ruapehu and White Island - marking its southern and northern end, respectively. Due to oblique subduction, the volcanic front rotates clockwise over time [Walcott, 1984; Wright \& Walcott, 1986]. The young TVZ belongs to the most active and productive silicic volcanic systems on Earth [Wilson et al., 1995]. The tectonic motion is expressed by normal faulting with an average orienta- 


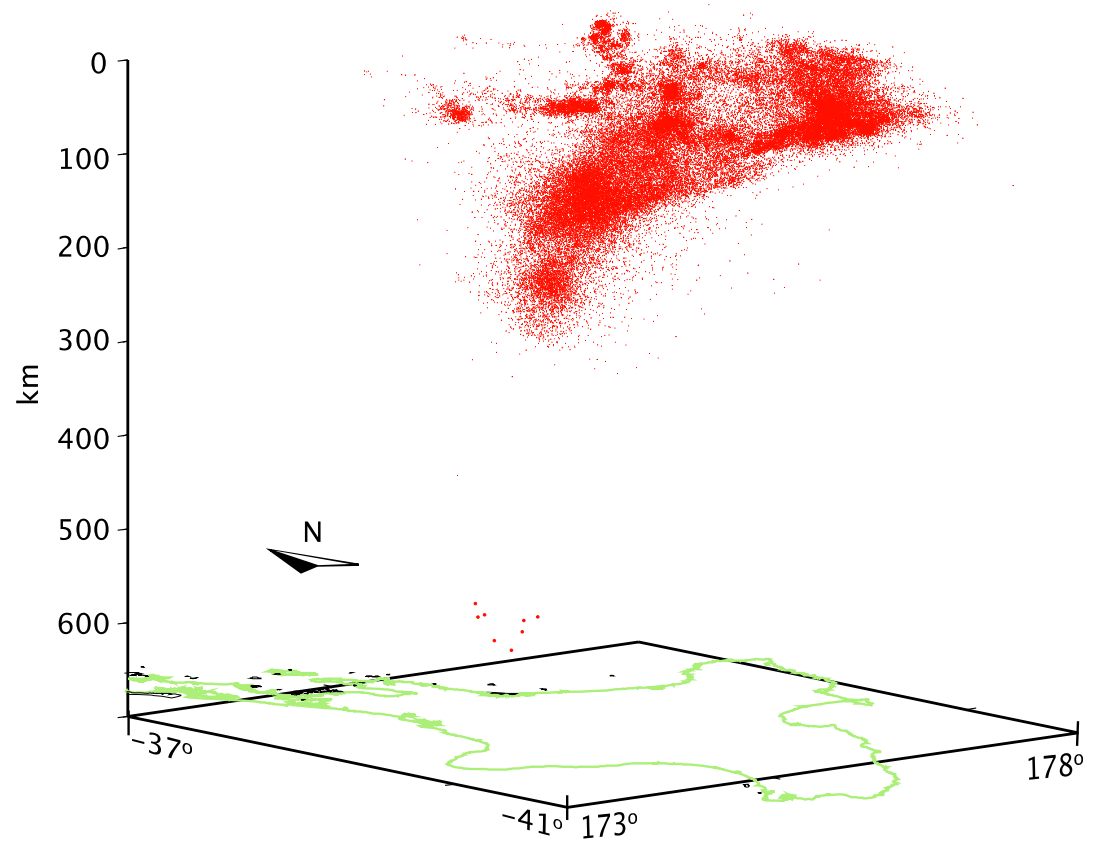

FIGURE 2.3 Three dimensional perspective view of earthquakes under central North Island. Earthquakes are taken from the GeoNet [2001] database covering the period from 01/01/1990 to 28/05/2008 for events larger than magnitude 2 in the rectangular map area. The few deep earthquakes under Mt. Taranaki are enhanced for better visualisation. Coast line of North Island in green.

tion perpendicular to the extension direction [Villamor \& Berryman, 2001; Lamarche et al., 2006].

Western central North Island, located in the far backarc, is currently tectonically quiet, with no measurable deformation happening [Wallace et al., 2004]. As part of the Australian plate, it moves northwards $\left(10^{\circ}\right)$ [Demets et al., 1990]. In contrast to the CVR, the most recent magmatic events in western North Island are potassic Pliocene and basaltic to andesitic Miocene volcanism [Briggs, 1986]. One outstanding exception to this pattern is the presently active andesitic Mt. Taranaki west of the CVR. This indicates that Northland-Waikato has been subjected to up to $18 \mathrm{Ma}$ of volcanism and thermal weakening of the lithosphere [Smith et al., 1989].

\subsubsection{Geophysical signature of crust and upper mantle}

Primary information about the mantle structure under central North Island comes from earthquake locations [e.g. Anderson \& Webb, 1994; Ansell \& Bannister, 1996]. The WaditiBenioff zone shows a shallow dipping slab under eastern central North Island, steepening to about 100-150 km depth under the middle of North Island (figure 2.3). Under the west coast, deep earthquakes mark the slab down to around $250 \mathrm{~km}$ and their alignment becomes almost vertical. However, below the Taranaki region earthquakes occur at depth of up to $600 \mathrm{~km}$ [Adams \& Ferris, 1976]. They have been interpreted as remains of previous subduction episodes [Smith et al., 1989]. High resolution upper mantle local earthquake tomogra- 


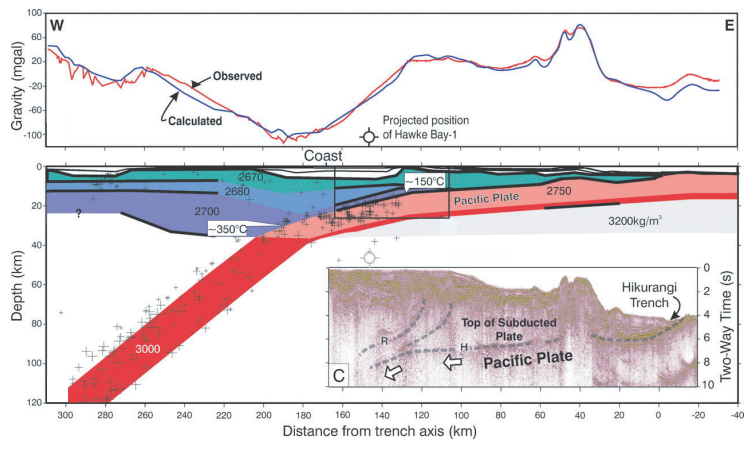

(a) Profile across the central North Island from Henrys et al. [2006]

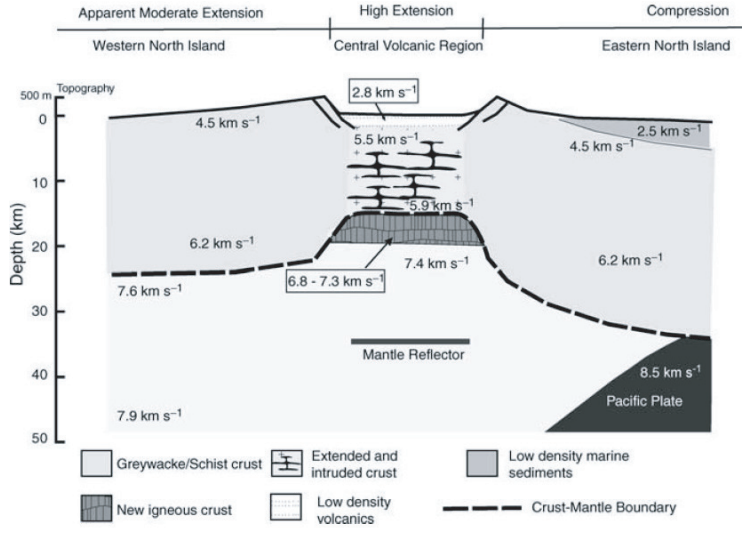

(b) Crust and upper mantle structure of central North Island from Stratford \& Stern [2006]

FIGURE 2.4 Crustal and upper mantle structure from active source seismic

phy [Reyners et al., 2006] completes and refines this image.

Receiver functions to identify more structural details across central North Island are only available down to about $60 \mathrm{~km}$ [Bannister et al., 2007]. The shallow top of the slab shows lower S-wave velocity than the overlying and underlying mantle, and is interpreted as the subducted crust. No distinct Moho is found west of CVR, where S-wave velocities increase gradually between 20 and $38 \mathrm{~km}$ depth.

Active seismology images more detailed shallow structure (figure 2.4). A sharp change in dip is suggested to occur at shallow depth of about $12 \mathrm{~km}$ (figure $2.5(\mathrm{a})$ ) and is interpreted to mark a change from stable to unstable slip regimes [Henrys et al., 2006]. These changes in dip of the slab can play an important role in the distribution of stress and fluid release for the mantle wedge. Stern \& Davey [1987] suggested thin continental crust of about $15 \mathrm{~km}$ under the CVR. But other studies give much larger values of about $30 \mathrm{~km}$ [Harrison \& White, 2006].

Tomography and active source seismology additionally provide information about velocity and attenuation structure. The subducted slab stands out with high P-wave velocities and low attenuation [Eberhart-Phillips et al., 2005]. As part of the western Pacific plate, it is assumed to be old (Cretaceous, about $120 \mathrm{Ma}$ ) and therefore dense and cold [Moores \& Twiss, 1995]. But age measurements of the currently subducting seafloor of the Hikurangi Plateau have not been made [Smith et al., 1989]. In the mantle wedge below the CVR, the influence of fluids and melts causes low P-wave velocities [Haines, 1979], high vp/vs ratios [Eberhart-Phillips et al., 2005] and high P-wave attenuation [Hatherton, 1970; Mooney, 1970]. Similar to the CVR, the backarc region in western central North Island also exhibits low P-wave velocities [Harrison \& White, 2004; Stern et al., 1987; Stratford \& Stern, 2004] and high attenuation [Hatherton, 1970; Mooney, 1970; Eberhart-Phillips et al., 2005].

Gravity and heat flow are additional geophysical parameters with distinguishing characteristics across the central North Island. A sharp gradient in the isostatic gravity anomaly 


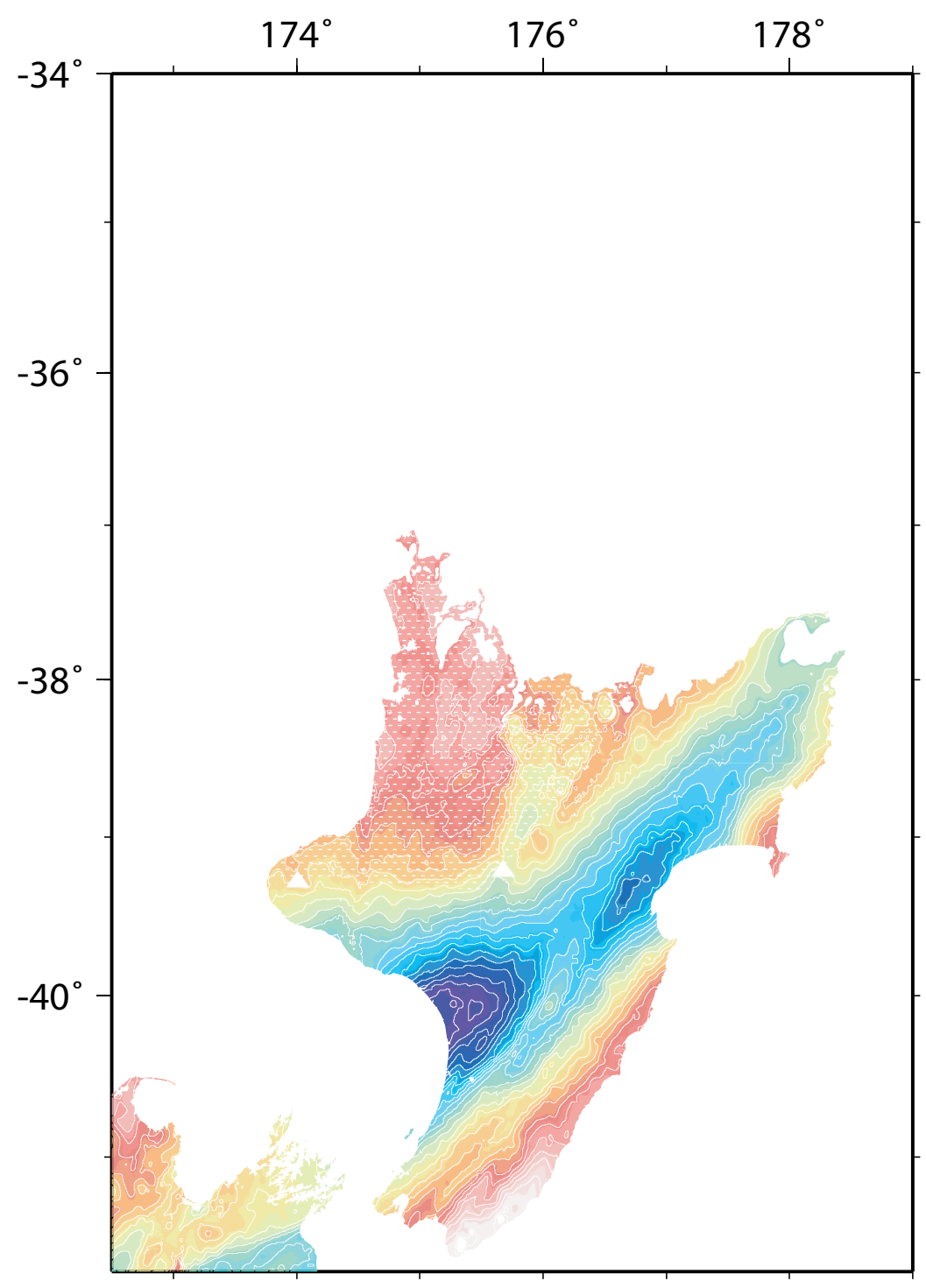


CVR is associated with exceptionally high heat output of about $840 \mathrm{~mW} / \mathrm{m}^{2}$ [Stern, 1987; Smith et al., 1989]. Generally in subduction zones, heat flow is expected to be low in the forearc, moderate in the backarc and high in the volcanic region [Peacock, 1996; van Keken, 2003]. The elevated values in western central North Island and CVR are ascribed to rapid changes in tectonics from compression to extension with most of the crustal shortening in the Miocene occurring in the western central North Island [Stern et al., 2006].

The strong changes in physical properties divide North Island into distinct regions. The boundaries of the regions are marked by the eastern boundary of the CVR, a line connecting Mt. Ruapehu and White Island (figure 2.5). The other boundary line is marked by the two southernmost volcanoes, Mt. Ruapehu and Mt. Taranaki (the Taranaki-Ruapehu line) (figure 2.5). This line is also considered as the transition zone between extensional and compressional back-arc regimes [Stern et al., 2006]. This is supported by strain rates, which show a change from shortening south of the line to extension north of the line [Beaven \& Haines, 2001; Wallace et al., 2004].

The regions northwest of the boundary line, the western central North Island and the CVR, exhibit characteristics like high heat flow [Pandey, 1981; Smith et al., 1989], positive gravity anomalies [Reilly et al., 1977], high attenuation [e.g. Mooney, 1970; Salmon et al., 2005], low velocities [Haines, 1979; Seward et al., 2008] and thin crust [e.g. Bannister et al., 2004; Stern \& Davey, 1987, 1989; Stratford \& Stern, 2004]. They are inferred to be caused by thermally disturbed, low-density mantle and thinned crust. The low density mantle can provide the buoyancy to cause the uplift of the elevated thin crust in western and central North Island [Horspool et al., 2006; Pulford \& Stern, 2004]. The changes in crustal thickness are thought to influence mantle flow pattern [Reyners et al., 2007]. A recent hypothesis suggests a Rayleigh-Taylor instability to cause of the anomalous characteristics of the western central North Island by convective removal of mantle lithosphere and parts of the crust [Stern et al., 2006]. 


\subsection{The upper mantle and subduction zone: Conditions and mate- rials}

This section gives a general introduction into the temperature and pressure conditions and the materials found in the top $400 \mathrm{~km}$ of the Earth, i.e. the crust and upper mantle.

\subsubsection{Temperature and pressure}

The properties of all mantle rocks are a function of temperature, pressure and water content. For the upper mantle, the focus area of this study, pressure varies between 0 and about 30 GPa [Dziewonski \& Anderson, 1981] (figure 2.6). The inflence of water will be discussed in section 2.2.3.

The governing factors of subduction zone thermal structure are the cold downgoing slab and the hot mantle wedge (figure 2.6). Details of the thermal structure depend on convergence rate, age and thermal structure of the incoming lithosphere, slab geometry and the mantle wedge flow field [Peacock, 2003]. Shear stress (frictional heating) along the top of the descending slab, mantle flow and water content can have additional influence [Peacock, 1996]. Thus, subduction zone thermal models differ in detail, depending on the model assumptions [cf. numerous recent publications, e.g. Currie et al., 2004]. Variations on the order of $100^{\circ} \mathrm{C}$ between different mantle wedge regions are inferred in seismological and petrological studies [Wiens et al., 2006, 2008].

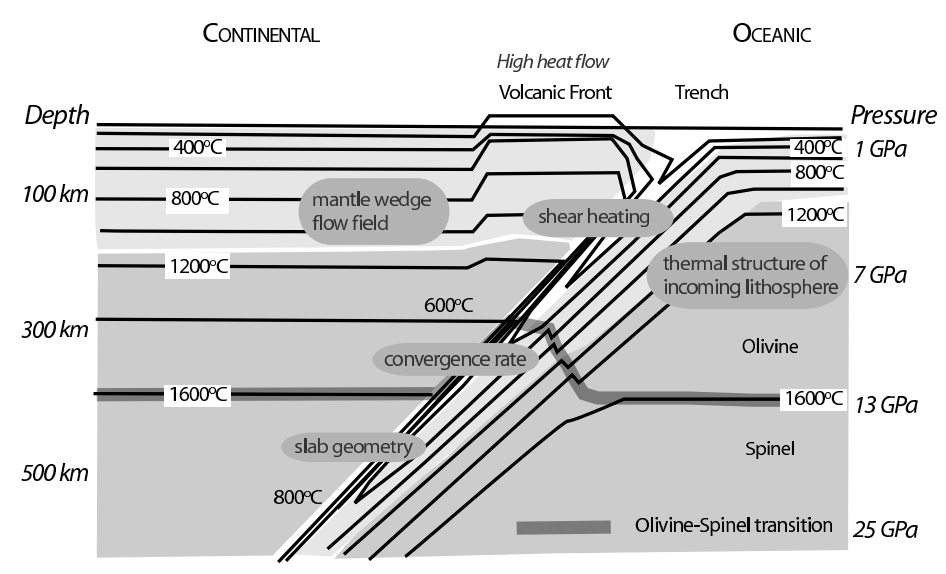

FIGURE 2.6 Temperature and pressure conditions in a subduction zone; also given are parameters with strong influence on the temperature field [after Turcotte \& Schubert, 2002; Peacock, 2003].

\subsubsection{Subduction zone materials}

The upper mantle of the Earth, down to about $410 \mathrm{~km}$, consists of mainly four important mineral phases: olivine, enstatite (orthopyroxene), clinopyroxene (diopside) and garnet 
[Ringwood, 1975]. They build rocks such as gabbro and peridotite. Gabbro is more abundant in oceanic crust at the boundary with the mantle [Anderson, 1989]. Peridotite is the main rock of the upper mantle (above $400 \mathrm{~km}$ ) [Anderson, 1989].

Oceanic plates consist of up to about $100 \mathrm{~km}$ of peridotite covered by a thin crust [Dziewonski \& Anderson, 1981]; the crust, commonly about 5 to $10 \mathrm{~km}$ thick, consists of basalt, gabbro, and minor sediments [Anderson, 1989]. Oceanic plates are usually subducted back into the mantle in subduction zones. However, pieces can be emplaced into or overthrust on continental crust, a process called obduction; the emplacement may occur during orogenies, during continent-continent or continent-island arc collisions [Turcotte \& Schubert, 2002]. The pieces of oceanic crust within continental crust are referred to as ophiolites ; typical ophiolites consist mostly of peridotite plus associated gabbro and/or basalt.

Peridotite contains at least $40 \%$ of olivine, the other parts are orthopyroxe and clinopyroxene [Anderson, 1989]. The high percentage of iron and magnesium in olivine creates a very dense rock. Peridotite gets altered in the subduction zone [Moores \& Twiss, 1995], as it loses elements like iron during the melting process. This causes the olivine content to increase while the pyroxene content decreases. In the presence of volatile components, the solidus curve of peridotite is lowered. Liquids derived from it are then more andesitic. Andesites form the most significant component of subduction-related magmatism [Wyllie, 1992]. The rocks of the peridotite family are uncommon at the surface and are highly unstable, because olivine reacts quickly with water at typical temperatures of the upper crust and at the Earth's surface [Christensen, 2004]. Xenoliths are fragments of peridotites, which were carried up by magmas from the mantle [Twiss \& Moores, 1997]. Basalt and kimberlite are examples of magmatic compositions that commonly include peridotite xenoliths.

Olivine is the most abandoned mineral of the upper mantle $(\sim 60-70 \%)$. It is also the weakest upper mantle mineral [Bai et al., 1991]. Deeper than $400 \mathrm{~km}$, olivine will change into the higher-pressure mineral Wadsleyite $(410 \mathrm{~km})$ and Ringwoodite $(520 \mathrm{~km})$ [Stein \& Wysession, 2003].

Serpentinites are metamorphic alterations of olivine and pyroxene abundant in surface outcrops of peridotite [Moores \& Twiss, 1995]. The physical properties of serpentines vary widely [Christensen, 2004]. Samples of the oceanic crust and the uppermost mantle of ocean basins document that ultramafic rocks there commonly contain abundant serpentine [Fowler, 2001]. Antigorite is the polymorph of serpentine that most commonly forms during metamorphism of wet ultramafic rocks and is stable to pressures of around $8 \mathrm{GPa}$ [Ulmer \& Trommsdorff, 1995]. As it can store large amounts of water (13 wt.\%), antigorite may play an important role in transporting water into subduction zones and in the subsequent release of water to create melt.

Sediments get dragged down into the subduction factory on top of the subducting slab. The thickness of the sedimentary layer is generally on the order of a few hundreds meters, but can vary considerably [Poli \& Schmidt, 2002]. 
A variety of trace elements can be found in subduction zones. Particularly abundant is chlorite, which gets into the subduction zone with the sediments that have been in contact with seawater. Chlorite strongly influences the properties of the wedge [Keppler, 1996]. It is stable to about $750-800^{\circ} \mathrm{C}$ [Poli \& Schmidt, 2002].

\subsubsection{Water}

The presence of water in subduction zones is established by the dehydration of water enriched sediments, metamorphosed basaltic oceanic crust and the subducting slab [Tatsumi \& Eggins, 1995]. Furthermore, seawater can penetrate to several kilometres depth [Stern, 2002]. The concentration of water in the slab decreases with distance from the trench.

Volatiles dissociate during the heating process of carbonates and hydrated minerals [Wyllie, 1992]. The ascending water then drives melting in the wedge [Grove et al., 2006]. This positive correlation between water concentration and the extent of melting in subduction zones is not observed in mid-ocean ridges [Kelley et al., 2006], where water content is usually lower.

Most of the volatiles in the upper mantle are not "free", but bound to the structure of nominally anhydrous minerals [Bolfan-Casanova, 2005]. But water also has a high solubility in melt [Hirth \& Kohlstedt, 1996]. Free water can only exist if the storage capacity of the material is reached. The mantle can only dissolve low amounts of water [Bolfan-Casanova, 2005]. Excess water rises and gets absorbed in the overriding lithosphere. Up to $0.5 \mathrm{wt}$. $\%$ water are reported in backarc basins [Kelley et al., 2006]. This is on the order of the storage capacity of upper mantle phases [Hirschmann et al., 2005]. Solubility increases with temperature and pressure [Manning, 1996]. The fluid and the melt phase become identical at P-T conditions higher than typical for subducting slabs. Therefore the amount of volatiles to about 3-4 GPa is assumed to be relatively low [Poli \& Schmidt, 2002]. Chlorite and antigorite are the critical volatile-bearing phases in the slab up to 5 GPa [Iwamori, 1998]. During serpentinisation, mantle wedge rocks can absorb up to $8 \mathrm{wt}$.\% of water [Connolly, 2005]

The importance of water in the subduction process lies in its ability to lower the solidus of mantle phases and increase melt productivity [Ulmer, 2001; Kelley et al., 2006]. It also has strong influence on the formation of volcanoes and earthquakes [e.g. Davies \& Stevenson, 1992]. The addition of fluids can reduce friction coefficients and lead to decoupling of the slab and mantle wedge [Arcay et al., 2006]. Fluids modify physical properties, causing an increase in melt connectivity and decrease in density and viscosity, which leads to highly efficient separation of fluid-rich melts from their sources. The impact of water on the elastic properties of a medium is significantly larger than any addition of other minerals. However, measurements are rare and only available for hydrous Ringwoodite [Jacobsen, 2006; Wang et al., 2003]. Xenolith samples show a wide range of water content in olivine [e.g. Jacobsen, 2006]. Differences could be due to water loss or gain during the ascent of the xenoliths. Generally, water reduces the bulk modulus of a mineral and increases its pressure deriva- 
tive. Effects on the shear modulus of hydrous olivine have not been measured yet, and therefore effects on shear velocities are unknown.

\subsubsection{Melt}

The upper mantle, at depths of 150 to $400 \mathrm{~km}$, is assumed to always be near solidus and therefore near melting [Brown et al., 1992]. Different processes can cause material to reach the state of melting; (1) Raising the temperature is the most obvious. However, due to the slowness of conductive heating, it does not prevail in the mantle [Gill, 1981]. (2) Decompression causes melting, as the solidus and liquidus temperature decreases when pressure drops. Thus, bringing material closer to the surfaces allows it to melt [e.g. Asimow et al., 1995, 2001]. As the solidus is a function of pressure and temperature, it is also a function of place. The incomplete melting process of an initial rock, composed of different minerals, is called partial melting. (3) Lowering the melting point of the material by changing its composition is another effective process to produce melt. This is the main process for melting in subduction zones, where elements incompatible with the slab (such as water, sodium and potassium) [Pearce \& Peate, 1995; Elkins-Tanton \& Grove, 2003] rise and mix with the mantle wedge material. The addition of oxides can reduce the melting temperature by about $100^{\circ} \mathrm{C}$ [Hirschmann et al., 1999]. Water has the additional effect of increasing the melt productivity [Kelley et al., 2006].

The major melt source in the upper mantle is peridotite [Wyllie, 1992]. The melt has basaltic composition, leaving behind residuals of olivine, orthopyroxene and some clinopyroxene. A typical example for these "leftovers" is harzburgite. Melt from the slab is assumed to only provide minor contributions to the overall melt content [Poli \& Schmidt, 2002].

Properties of melt vary over a broad range and strongly depend on composition, pressure and temperature [Dingwell, 2007]. The density of partially molten basalt is generally lower than of the crystalline matrix [Turcotte \& Schubert, 2002]. This density difference causes the separation of the melt from its mantle source, referred to as melt segregation or compaction. After segregation, melt and matrix can remain in thermodynamic equilibrium or the melt gets extracted and rises independently, cooling adiabatically on its way up [Gill, 1981]. Velocities of melt migration are at least 1-10 m/yr [Kelemen et al., 1997; Hawkesworth et al., 1997]. To travel upwards melt has to migrate faster than the plate is moving away and melt velocities much faster than plate speeds lead to vertical melt channels [Braun \& Sohn, 2003]. In the mantle wedge, melt is assumed to migrate through porous flow [e.g. Gaetani \& Grove, 1998] and therefore slowly enough to reach a local equilibrium (at the channel walls and internally) with the surrounding mantle [Aharonov et al., 1995]. Water might take an additional role in lowering the viscosity of the melt and faciliating vertical flow [Kelley et al., 2006]. Melting stops when it enters the lithosphere, as melt gets in contact with colder materials and heat loss occurs through conduction. However, cooling by conduction is slow compared to the melt rising velocities, so that the melt needs to be arrested to cool. 
Melt is often inferred to exist in isolated pockets, along grain edges in tubular inclusions or along grain faces in disk-like inclusions [Schmeling, 1985]. Faul et al. [1994] found disk like inclusions with aspect ratios of 0.05 in peridotite. Basaltic melt in low fractions (about $3 \%$ ) in olivine mainly occurs in low aspect ratio, disk-shaped inclusions. About $10 \%$ of the melt are found in interconnected channels around the grains [Faul, 1997]. As long as the two systems do not interconnect, permeability and flow are low. The interconnectivity of melt is controlled by the melt inclusion geometry [Wyllie, 1992], so that also small amounts of melt can be interconnected.

While in a stress-free environment melt gathers around grains, under deviatoric stress melt forms highly aligned pockets with preferred orientations subparallel to the stress [Kohlstedt \& Zimmerman, 1996; Daines \& Kohlstedt, 1997; Zimmerman et al., 1999]. Segregation and alignment can occur over scales larger than grain scale and form melt-rich layers or bands separated by depleted regions [Holtzman et al., 2003b]. These bands localise deformations and provide high permeability pathways for melt migration. The preferred alignment of features such as melt pockets or bands causes some material properties, such as seismic attenuation and velocity to become direction dependent [Takei, 2001, 2002] (cf. chapter 3). 


\section{Chapter 3}

\section{Seismic Anisotropy}

This chapter gives an introduction into the basic theory of seismic anisotropy. Generally, the term anisotropy describes the variation of a material property with direction. Seismic anisotropy is therefore the variation of the seismic velocity with propagation direction through a medium. The first section will introduce the mathematical representation of anisotropy, followed by the equations needed to describe wave propagation in anisotropic media. The chapter finishes with an overview of causes for seismic anisotropy in the (upper) Earth.

\subsection{Mathematical description of anisotropy - The elastic tensor}

Similar to symmetry structures of crystals, the anisotropic symmetry of any system can be described mathematically by a fourth-order elastic stiffness tensor $C_{i j k l}$. It describes the relationship between the second-order stress $\epsilon_{k l}$ and strain tensors $\sigma_{i j}$. Deformation experiments show that for small forces each component of strain is linearly proportional to every component of the applied stress. This relationship is called Hooke's law [e.g. Stein \& Wysession, 2003]:

$$
\sigma_{i j}=C_{i j k l} \varepsilon_{k l}
$$

for $i, j, k, l=1,2,3$. The summation applies over the subscripts $k$ and $l$. $C_{i j k l}$ represents the fourth-order tensor of elastic moduli and defines the material properties of the medium. In its most general form, it has $3^{4}=81$ terms.

However, the symmetry of the system means that many of the 81 elements of the elastic tensor are not independent. Symmetry of stress and strain tensor (Voigt symmetry, $C_{i j k l}=C_{j i k l}=C_{i j l k}=C_{j i l k}$ ) reduces its number of independent coefficients to 36 . The consideration of strain energy density function (the existence of a unique strain-energy potential $C_{i j k l}=C_{k l i j}$ ) reduces the number of independent coefficients to 21 . This is the maxi- 


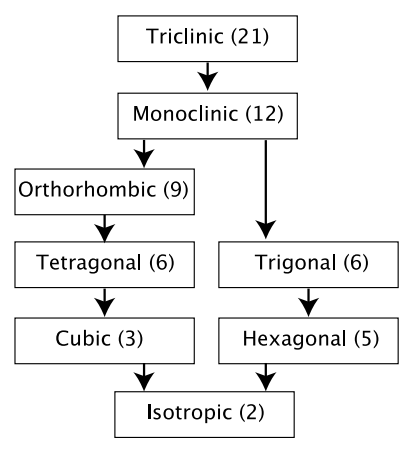

FIGURE 3.1 Hierarchy of symmetry classes All media are triclinic, other symmetries are of higher order. The number of independent elastic constants in the symmetry class is given in brackets.

mum number of elastic constants that any medium can have and defines the most general anisotropic symmetry (figure 3.1). Crystallographic symmetries further reduce the number of independent coefficients [Nye, 1985].

Because of the difficulties to print a four-dimensional tensor on paper, the Voigt notation [Nye, 1985] is often applied to express $C_{i j k l}$ with $i, j, k, l=1,2,3$ with the symmetric secondorder matrix $C_{m n}$ with $m, n=1-6$. The reduced subscripts are defined as:

$1=(1,1), 2=(2,2), 3=(3,3), 4=(2,3)=(3,2), 5=(1,3)=(3,1), 6=(1,2)=(2,1)$. Symmetry applies in the sense that $C_{i j}=C_{j i}$. The diagonal constants with $i \leq 3$ are often referred to as longitudal elastic constants, the ones with $i \geq 4$ as shear elastic constants [Karki et al., 2001].

For rocks the most common symmetries are:

- Triclinic: 21 coefficients (all media are triclinic)

$$
C_{i j}=\left(\begin{array}{llllll}
c_{1111} & c_{1122} & c_{1133} & c_{1123} & c_{1113} & c_{1112} \\
c_{2211} & c_{2222} & c_{2233} & c_{2223} & c_{2213} & c_{2212} \\
c_{3311} & c_{3322} & c_{3333} & c_{3323} & c_{3313} & c_{3312} \\
c_{2311} & c_{2322} & c_{2333} & c_{2323} & c_{2313} & c_{2312} \\
c_{1311} & c_{1322} & c_{1333} & c_{1323} & c_{1313} & c_{1312} \\
c_{1211} & c_{1222} & c_{1233} & c_{1223} & c_{1213} & c_{1212}
\end{array}\right)
$$

- Orthorhombic: symmetry about three orthogonal planes, 9 coefficients, $c_{11}, c_{22}, c_{33}$, $c_{44}, c_{55}, c_{66}, c_{12}=c_{21}, c_{13}=c_{31}, c_{23}=c_{32}$

$$
C_{i j}=\left(\begin{array}{llllll}
\mathrm{a} & \mathrm{b} & \mathrm{c} & 0 & 0 & 0 \\
\mathrm{~b} & \mathrm{a} & \mathrm{e} & 0 & 0 & 0 \\
\mathrm{c} & \mathrm{e} & \mathrm{f} & 0 & 0 & 0 \\
0 & 0 & 0 & \mathrm{~g} & 0 & 0 \\
0 & 0 & 0 & 0 & \mathrm{~h} & 0 \\
0 & 0 & 0 & 0 & 0 & \mathrm{i}
\end{array}\right)
$$

In this case, the planes of symmetry are perpendicular to the Cartesian axes $x_{1}, x_{2}$ and 


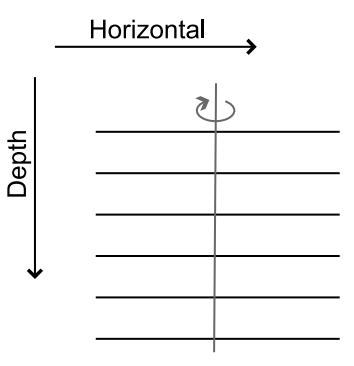

a) Transverse isotropy with vertical symmetry axis

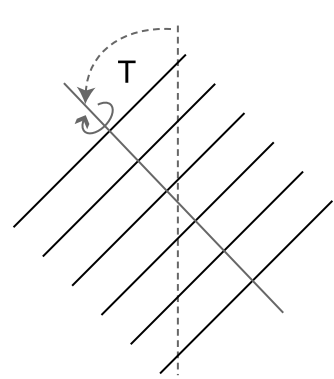

b) Transverse isotropy with tilted symmetry axis

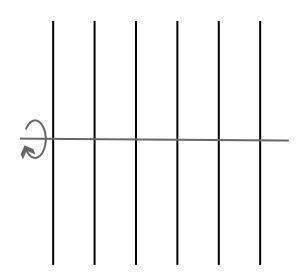

c) Azimuthal anisotropy with horizontal symmetry axis

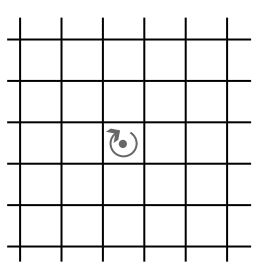

d) Orthogonal symmetry

FIGURE 3.2 The most common anisotropic symmetries in the Earth. The grey line marks the axis of symmetry. $T$ is the dip angle for tilted symmetry axis.

$x_{3}$.

- Hexagonal: axial symmetry, 5 coefficients defined as Love parameters: $A=c_{11}=c_{22}$, $C=c_{33}, F=c_{13}=c_{23}, L=c_{44}=c_{55}, N=c_{66}, c_{12}=c_{21}=A-2 N$ or Thomsen parameters (commonly used in exploration seismology, $c f$. appendix F.2)

$$
C_{i j}=\left(\begin{array}{cccccc}
\mathrm{A} & \mathrm{A}-2 \mathrm{~N} & \mathrm{~F} & 0 & 0 & 0 \\
\mathrm{~A}-2 \mathrm{~N} & \mathrm{~A} & \mathrm{~F} & 0 & 0 & 0 \\
\mathrm{~F} & \mathrm{~F} & \mathrm{C} & 0 & 0 & 0 \\
0 & 0 & 0 & \mathrm{~L} & 0 & 0 \\
0 & 0 & 0 & 0 & \mathrm{~L} & 0 \\
0 & 0 & 0 & 0 & 0 & \mathrm{~N}
\end{array}\right)
$$

- Cubic: 3 coefficients, $c_{11}=c_{22}=c_{33}, c_{44}=c_{55}=c_{66}, c_{12}=c_{23}=c_{13}$

- Isotropic: 2 coefficients. The most common isotropic coefficients are known as Lamé constants $\lambda\left(=c_{12}=c_{23}=c_{13}\right)$ and the shear modulus $\mu\left(=c_{44}=c_{55}=c_{66}\right) ;\left(c_{11}=c_{22}=\right.$ $\left.c_{33}=\lambda+2 \mu\right)$. But any combinations of the Lamé constants, i.e. the Young's modulus $E$, the bulk modulus or incompressibility $K$, the Poisson ratio $\nu$ and the P-wave modulus $M$ can be used to describe isotropic elastic properties. The relationships between them are well-known and can be found in many text books [e.g. Mavko et al., 1998]. As a common first approximation the Earth is described as a Poisson solid with $\mu=\lambda$ and therefore $\nu=0.25$.

The most commonly used assumptions for seismic anisotropy symmetries in the Earth are orthorhombic or hexagonal with vertical symmetry axes, also called transverse isotropy (figure 3.2). If velocities are direction dependent in the horizontal plane, this is called azimuthal anisotropy. 


\subsection{Wave propagation in anisotropic media - Basic equations}

To understand the relationship between propagation direction and displacement in anisotropic media, the basic equations describing body wave propagation in such a medium are introduced. The derivations summarised here basically follow Babuška \& Cara [1991].

For a continuous, homogenous medium with a density $\rho$, the three-dimensional elastodynamic equation of motion can be written in terms of displacement $\left(u_{i}\right)$ as function of time $(t)$ :

$$
\rho \frac{\partial^{2} u_{i}}{\partial t^{2}}=\frac{\partial \sigma_{i j}}{\partial x_{j}}, i=1,2,3
$$

Inserting Hooke's law (equation 3.1) into the wave equation above (equation 3.5) and expressing the strain as

$$
\epsilon_{k l}=\frac{1}{2}\left(\frac{\partial u_{l}}{\partial x_{k}}+\frac{\partial u_{k}}{\partial x_{l}}\right)
$$

leads to the wave equation in an anisotropic medium:

$$
\rho\left(\frac{\partial^{2} u_{i}}{\partial t^{2}}\right)=C_{i j k l}\left(\frac{\partial^{2} u_{l}}{\partial x_{j} x_{k}}\right)
$$

The displacement of a monochromatic plane wave can be written as [Babuška \& Cara, 1991]:

$$
\vec{u}=\vec{a} f\left(t-\frac{\vec{n} \cdot \vec{x}}{c}\right)
$$

where $\vec{a}$ is the amplitude vector, which gives the polarisation direction and magnitude of the particle motion, $c$ is the phase velocity and $\vec{n}$ is the normal vector pointing into propagation direction at coordinates $\vec{x}$.

Inserting this equation (3.8) and its derivatives into the wave equation (equation 3.7) and using the Kronecker delta function for $a_{i}=\delta_{i l} a_{l}$, the so-called Christoffel equation [Musgrave, 1970] is derived:

$$
\left(m_{i l}-c^{2} \delta_{i l}\right) a_{l}=0
$$

with the components of the Christoffel Tensor $\underline{M}$ being:

$$
m_{i l}=\frac{C_{i j k l} n_{j} n_{k}}{\rho}
$$

The Christoffel equation is a typical eigenvalue problem. Its solutions give the squared phase velocities (i.e. eigenvalues) and the polarisation direction (i.e. the eigenvector corresponding to each eigenvalue) for a wave propagating along an arbitrary direction $\vec{n}$. The problem has three solutions, corresponding to a quasi longitudal wave $(\mathrm{P})$ polarised nearly parallel to the propagation direction and two quasi shear-waves polarised nearly perpendicular to propagation (quasi-transverse SV and quasi-longitudal SH). This illustrates the de- 
pendence of the shear-wave velocity on polarisation and propagation direction. Because of the symmetry of the Christoffel matrix $\underline{M}$, the polarisation directions are mutually perpendicular, but the waves will usually not be purely longitudinally or transversally polarised. For small anisotropy, the angles between propagation and polarisation directions are small; hence, the waves are termed quasi-P or quasi-S.

To analytically solve for the eigenvalues and eigenvectors, knowledge of the complete elastic tensor is required. The solutions of the Christoffel matrix provide the velocities for seismic waves for the direction of propagation through the medium. The easiest solutions exist for propagation along the symmetry axis. For example, for seismic waves travelling along the $x_{1}$-axes, the velocities can be expressed as the following:

\section{For isotropy:}

$$
\begin{aligned}
& v_{P}=\sqrt{\frac{c_{11}}{\rho}}=\sqrt{\frac{\lambda+2 \mu}{\rho}} \\
& v_{S}=\sqrt{\frac{c_{44}}{\rho}}=\sqrt{\frac{\mu}{\rho}}
\end{aligned}
$$

For hexagonal anisotropy: There are three independent solutions, a quasi-longitudal P-wave velocity, a transverse or pure shear-wave velocity and a quasi-transverse or quasi shear-wave velocity for each propagation direction. With $\theta$ being the angle between the wave vector and the axis of symmetry, the velocities can be obtained by:

$$
\begin{aligned}
\text { quasi-longitudal } & v_{P}=\sqrt{\frac{c_{11} \sin ^{2} \theta+c_{33} \cos ^{2} \theta+c_{44}+\sqrt{M}}{2 \rho}} \\
\text { quasi-shear } & v_{S V}=\sqrt{\frac{c_{11} \sin ^{2} \theta+c_{33} \cos ^{2} \theta+c_{44}-\sqrt{M}}{2 \rho}} \\
\text { pure-shear } & v_{S H}=\sqrt{\frac{c_{66} \sin ^{2} \theta+c_{44} \cos ^{2} \theta}{\rho}}
\end{aligned}
$$

with

$$
M=\left[\left(c_{11}-c_{44}\right) \sin ^{2} \theta-\left(c_{33}-c_{44}\right) \cos ^{2} \theta\right]^{2}+\left(c_{13}+c_{44}\right)^{2} \sin ^{2} 2 \theta
$$

In the special case of propagation along the symmetry axis at horizontal incidence $\theta=90^{\circ}$, this reduces to:

$$
\begin{aligned}
& v_{P}=\sqrt{\frac{c_{11}}{\rho}}=\sqrt{\frac{A}{\rho}} \\
& v_{S V}=\sqrt{\frac{c_{44}}{\rho}}=\sqrt{\frac{L}{\rho}} \\
& v_{S H}=\sqrt{\frac{c_{66}}{\rho}}=\sqrt{\frac{N}{\rho}}
\end{aligned}
$$




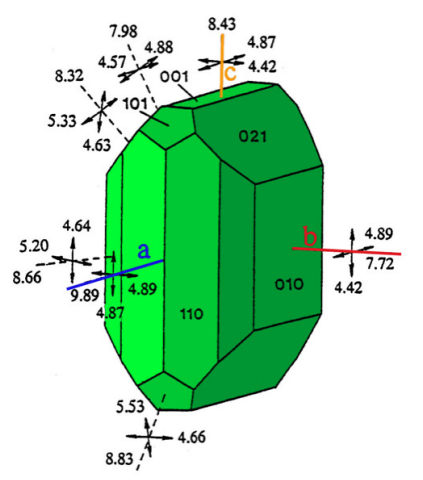

FIGURE 3.3 Direction dependent compressional and shear-wave velocities in an olivine monocrystal. Major crystallographic axes are indicated in colour; the fast [100] aaxis is blue. Velocities are given in $\mathrm{km} / \mathrm{s}$.

However, for seismic waves travelling along the other symmetry axes at vertical incidence $\theta=0^{\circ}$, two of the three eigenvalues are degenerate:

$$
\begin{aligned}
v_{P} & =\sqrt{\frac{c_{33}}{\rho}}=\sqrt{\frac{C}{\rho}} \\
v_{S H} & =\sqrt{\frac{c_{44}}{\rho}}=\sqrt{\frac{L}{\rho}} \\
v_{S V} & =\sqrt{\frac{c_{44}}{\rho}}=\sqrt{\frac{L}{\rho}}
\end{aligned}
$$

This means that there is only one shear-wave polarised perpendicular to the symmetry axes and it travels with the velocity of the symmetry axis. Note that the largest or smallest shear-wave velocities are not necessary along a structural or a symmetry axis (figures 3.3 and 3.5).

The direction dependence of velocities causes a single seismic shear-wave with initial polarisation propagating through an anisotropic medium to split into two mutually perpendicular waves polarised along the faster and slower planes of the medium (figure 3.4). The shapes of those two waves are identical, but the velocities and amplitudes depend on the initial polarisation with respect to the fast and slow orientations. If the incoming polarisation is along the fast or slow axis, the wave will not split. Therefore, when analysing anisotropic structures, it is crucial to use multiple rays that have traversed the same raypath but with different incoming polarisation.

A value to quantify the anisotropy given by a fast $\left(v_{\text {fast }}\right)$ and a slow $\left(v_{\text {slow }}\right)$ quasi wave speed along a raypath is the percentage anisotropy. It is defined as:

$$
k=100 \frac{v_{\text {fast }}-v_{\text {slow }}}{\frac{v_{\text {fast }}+v_{\text {slow }}}{2}}=200 \frac{v_{\text {fast }}-v_{\text {slow }}}{v_{\text {fast }}+v_{\text {slow }}}=100 \frac{v_{\text {fast }}-v_{\text {slow }}}{\bar{v}_{S}}
$$

with $\overline{v_{S}}=\frac{v_{\text {fast }}+v_{\text {slow }}}{2}$.

Therefore, the percentage anisotropy $k$ depends on the propagation direction. This results in a non-spherical wavefront and in group velocities different from phase velocities [e.g. 


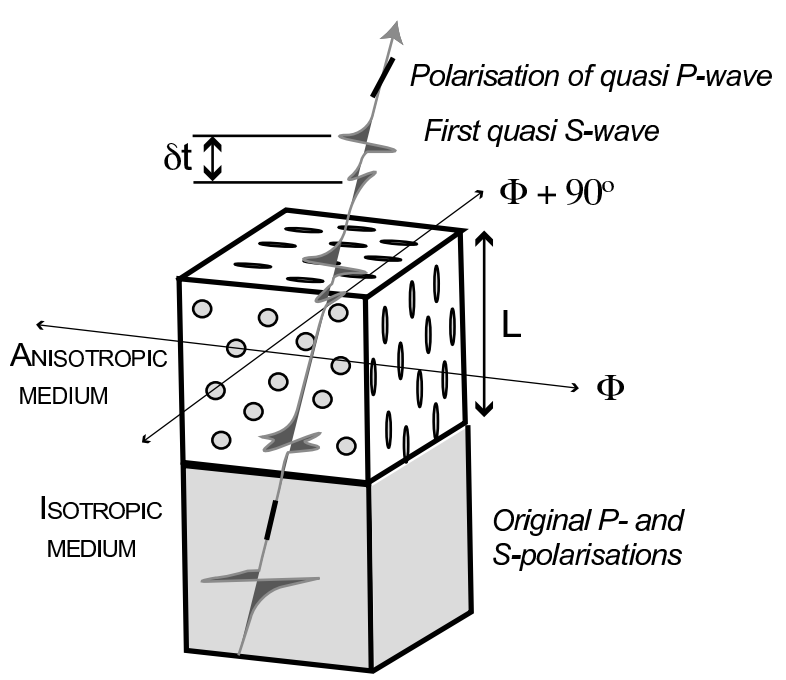

FIGURE 3.4 Seismic waves travelling through an anisotropic medium. In the isotropic medium, the original polarisation of the P-wave (black solid line) is parallel to propagation direction, of the S-wave (grey wave) perpendicular to propagation direction. In the anisotropic medium the P-wave polarisation is not propagation parallel any more. After exiting, the S-wave is split by a delay time $\delta t$, with the first wave polarisation parallel the fast orientation $\Phi$ of the anisotropic medium. $L$ is the travel path length through the anisotropic medium.

Kendall, 2000]. Group velocity is the velocity with which energy travels radially outward from a point source in a homogenous elastic anisotropic medium, i.e. the velocity along the ray that defines the wavefront. Phase velocity is the velocity in the direction of the phase propagation vector, normal to the surface of constant phase. Common laboratory and seismological measurements provide group velocities [Karki et al., 2001].

\subsection{Measuring seismic anisotropy}

\subsubsection{Elastic parameters}

The elastic tensor manifests details about the anisotropy of a medium. Full elastic tensors for single crystals [e.g. Kumazawa \& Anderson, 1969; Isaak et al., 1992; Abramson et al., 1997] are determined using ultrasonic pulses over a broad temperature and pressure range [Karki et al., 2001]. The additionally derived temperature and pressure derivatives permit to extrapolate properties at mantle- or core-like conditions [e.g. Anderson \& Isaak, 1995; Isaak, 2001].

Generally, there is a trend for anisotropy to decrease with increasing pressure and with decreasing temperature [Silver et al., 1999]. Table A.1 gives an overview of some published full elastic tensors for different mantle materials.

Rocks are typically not made from a single kind of crystal, but from a composition of different materials. To calculate seismic anisotropy of several crystals as the effective macroscopic modulus of the aggregate, averaging techniques are used. They assume consistent grain size, shape, orientation or phase distribution [cf. Mainprice, 2007, for an overview]. The Reuss average [Reuss, 1929] is the volume average of local compliances under the assumption of constant stress equal to the macroscopic stress everywhere in the sample. It gives a lower bound for the moduli of the anisotropic medium. The Voigt average [Voigt, 


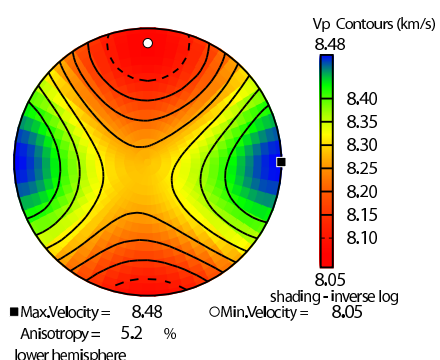
Anisotropy $=5.2$
lower hemisphere

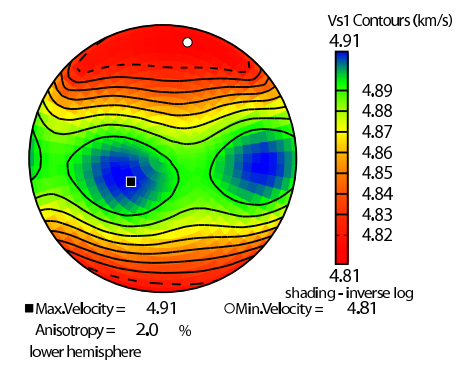

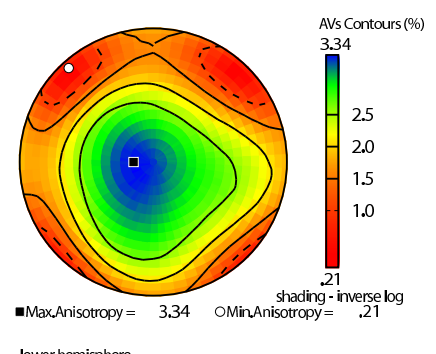

lower hemisphere

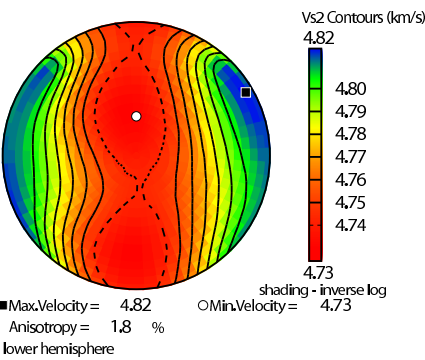

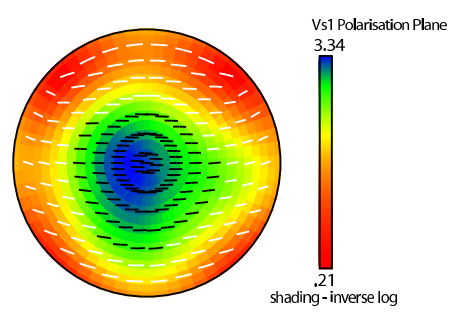

lower hemisphere

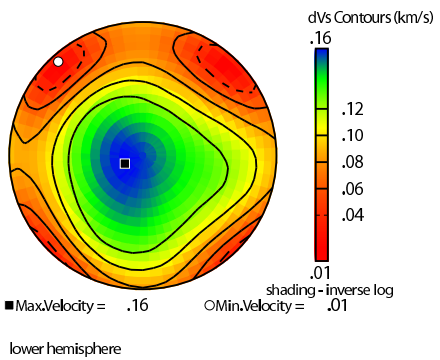

FIGURE 3.5 Calculated North Island xenolith average seismic properties, cf. also table A.1. Figure produced with CareWare software [Mainprice, 1990].

1928] is the volume average of local stiffnesses with orientation and volume fraction, defined for constant strain equal to the macroscopic strain everywhere in the sample. It gives an upper bound for the moduli of the anisotropic medium. Because the Reuss- and the Voigt-average yield lower and upper limits, their arithmetic (or geometric) mean tends to be a good compromise to estimate elastic properties of components [Hill, 1952]. It is called Voigt-Reuss-Hill-average and yields tensors close to experimentally derived properties [Barruol \& Kern, 1996]. However, minerals in a compound are usually not exactly aligned. In a polycrystal, the different crystal orientations can be measured (e.g. EBSD, Adams et al. [1993]) in terms of Euler angles. The exact macroscopic elastic parameters for monophase crystals are then determined by integration over all possible orientations and imaged in a stereographic plot (cf. example for North Island xenolith figure 3.5). For polyphase crystals, the macroscopic elastic tensor can only be determined if its texture is fully defined in terms of position, shape and orientation of the single grains. Some features, such as the presence of fluids and melt, are not preserved in recovered rock samples. Elastic constants under these special conditions need to be determined by other means, such as modelling [Kendall, 1994; Blackman \& Kendall, 1997; Mainprice, 1997].

\subsubsection{Shear-wave splitting parameters}

Shear-waves travelling in an arbitrary direction through an anisotropic medium split up into two components polarised quasi-perpendicular to each other and travelling with different speed (figure 3.4, section 3.1). Analogous to optics, this process is also referred to as birefringence. The polarisation of the faster travelling component $\Phi$ is characteristic for the 
anisotropic medium. After exiting the anisotropic medium, the two S-waves are separated by a delay time $\delta t$, which depends on the difference in speeds and the length of the travel path $L$ through the anisotropic medium:

$$
\delta t=L\left(\frac{1}{v_{S 1}}-\frac{1}{v_{S 2}}\right)
$$

where $v_{S 1}$ and $v_{S 2}$ are the faster and the slower quasi shear-wave speeds for the given propagation direction.

The delay time $\delta t$ and the fast polarisation $\Phi$ are called shear-wave splitting parameters or simply splitting parameters. In the presence of one single anisotropic layer along the raypath, the fast polarisation defines the fast orientation in the anisotropic medium and the delay time will be a direct measure for the thickness of the anisotropic layer. In the presence of several anisotropic layers along the travel path, the splitting parameters may appear as if they come from one single layer, but they carry collective information about all the traversed anisotropic layers. The measured splitting parameters are therefore often, in a more general sense, referred to as "apparent" splitting parameters [Silver \& Savage, 1994].

The percentage anisotropy (equation 3.23) allows comparing anisotropic strengths of media. In terms of delay time, following equations 3.23 and 3.24, it can be expressed as:

$$
k=\frac{\delta t}{L} v_{S} \cdot 100, \text { for } v_{S 1}-v_{S 2} \ll v_{S}
$$

where $v_{S}$ is the average $S$-wave speed for the propagation direction [Savage, 1999]. Note, that the anisotropic strength $k$ depends on the propagation direction and has to be distinguished from the intrinsic anisotropy, which is the percentage difference between the fastest and the slowest velocity and is therefore usually higher than the percent anisotropy (equation 3.23).

The observation of shear-wave splitting is the most unambiguous indicator of anisotropy, because the travel path of the two split shear-waves are almost identical. Characteristic particle motions and waveforms help to distinguish real "split" shear-waves from scattering. Shear-wave splitting will produce an elliptical particle motion. Silver \& Chan [1988] derive expressions for the radial and transverse component in case of transverse isotropy with horizontal symmetry axis:

$$
\begin{aligned}
& u_{r}(t)=s(t) \cos ^{2} \theta+s(t-\delta t) \sin ^{2} \theta \\
& u_{t}(t)=-\frac{1}{2}[s(t)-(s-\delta t)] \sin (2 \theta),
\end{aligned}
$$

with $s(t)$ being the waveform in the isotropic case and $\theta$ the angle between the fast and the radial component. This shows that the transverse component (equation 3.27) is approximately proportional to the time derivative of the radial component (equation 3.26). 


\subsection{Causes of seismic anisotropy in the Earth}

In a general sense, random structures cause isotropy; ordered heterogeneities of different scales cause anisotropy. The smallest scale of seismic anisotropy occurs in a single crystal. This pure form of anisotropy can be measured directly from rock samples in the laboratory (section 3.3.1). Larger scale anisotropy is caused by layered media or oriented inclusions with contrasting velocities. Geophysical measurements from seismic wave propagation can be sensitive to a large range of anisotropy. In the Earth, different regions exhibit different kinds of anisotropy.

The outermost core and most of lowermost mantle is assumed to be isotropic [Kaneshima \& Silver, 1995], with the exception of the lowermost boundary layer (D") [Kendall \& Silver, 1996] (figure 3.6). But anisotropy in the $\mathrm{D}^{\prime \prime}$ layer is inferred to be small [Restivo \& Helffrich, 2006]. The cause of the anisotropy in the $\mathrm{D}^{\prime \prime}$ layer and the inner core is still not well understood. The transition zones, where olivine phase transformations take place, are also the boundaries for different anisotropic regions.

Probably the highest anisotropy is observed in the uppermost parts of the Earth, the crust and the upper mantle. Causes for seismic anisotropy in the upper Earth, the main focus region of this study, are

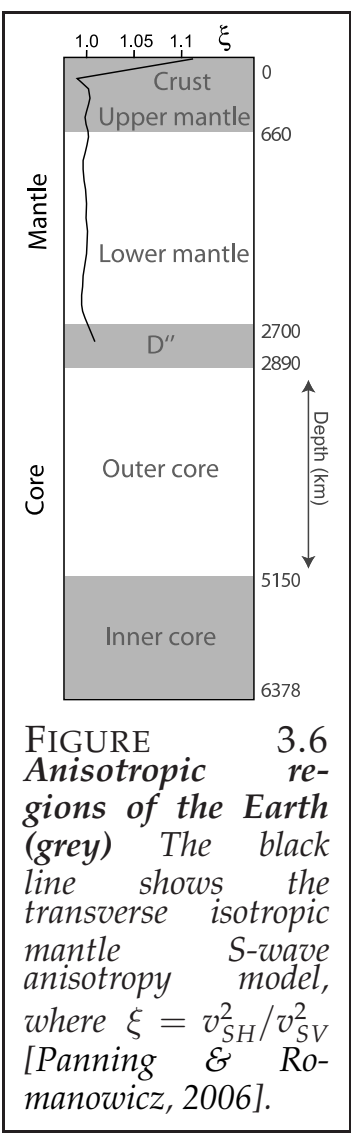
outlined in the following sections.

\subsubsection{Lattice-Preferred Orientation (LPO)}

Most minerals in the Earth are intrinsically anisotropic. In the crust deeper than $15 \mathrm{~km}$ the dominant anisotropic minerals are micas [Barruol \& Mainprice, 1993]; in the upper mantle it is olivine. Plastic deformation processes cause these minerals to align in a preferred orientation, a property called lattice-preferred orientation (LPO) or crystal-preferred orientation (CPO). If LPO occurs over a large volume, it generates or enhances anisotropy in the medium [e.g. Toriumi, 1984].

Two main deformation processes are responsible for generating LPO: dislocation creep and recrystallisation. In the upper mantle, the main boundary condition for deformation processes is simple shear, introduced by the horizontal movement of the lithosphere above the asthenosphere under sufficient coupling [Tommasi, 1998]. Pure shear is associated with extensional rifting [Vauchez et al., 2000]. Dislocation creep is the dominant high-temperature deformation mechanism at elevated stresses [Nicolas \& Christensen, 1987] and is insensitive to grain size [Li et al., 2003]. The LPO that develops depends on the active slip system (slip plane and slip direction). During dislocation creep, slip planes align with shear planes 


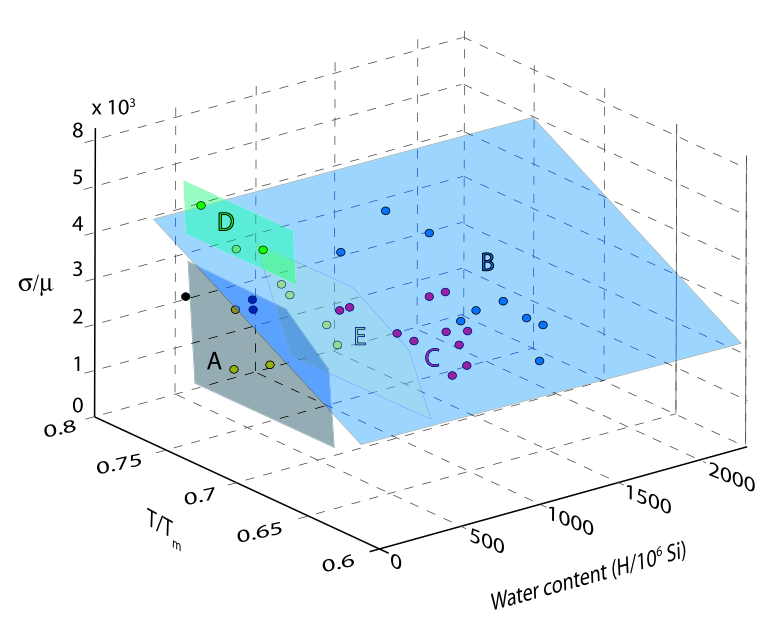

FIGURE 3.8 Different types of olivine LPO in a three-dimensional space of water content, normalised stress $\sigma / \mu$ and temperature $T / T_{m}$.

TABLE 3.1 Olivine fabrics, their abundance in rock samples [Ismail \& Mainprice, 1998] and their dominant slip systems

\begin{tabular}{|c|c|c|c|c|}
\hline A-type & B-type & C-type & D-type & E-type \\
\hline \hline $49.5 \%$ & $7.3 \%$ & $1.8 \%$ & $23.8 \%$ & $7.3 \%$ \\
\hline$[100](010)$ & {$[001](010)$} & {$[001](100)$} & {$[100](0 \mathrm{kl})$} & {$[100](001)$} \\
\hline
\end{tabular}

fabric develops for lithospheric conditions with modest temperature and stresses and low water content [Mehl et al., 2003].

With increasing water content and temperature (i.e. astenospheric conditions) the dominant fabric changes to E- and C-type [Katayama et al., 2004; Mizukami et al., 2004]. Under high stresses, D- and B-type fabric exists depending on the water content [Jung \& Karato, 2001b] (figure 3.8). B- and C-type olivine fabrics are distinctively different from other types as their fast axis does not align parallel to the flow direction [Katayama \& Karato, 2006]. These "wet" fabrics make up less than $10 \%$ of the olivine database. This could be due to water loss of the olivine on the way to the surface (low solubility of water in olivine) or, in the presence of melt, due to the absorption of the water into the melt (higher solubility of water in melt). However, the relative distribution of fabrics in field samples does not necessarily represent their abundance in the Earth [Tommasi et al., 2000].

These new olivine fabric types are of particular interest in subduction zones, where the presence of water is established by the dehydration of water enriched sediments, oceanic crust and lithosphere of the subducting slab (section 2.2.3).

The other important anisotropic phases in the upper mantle are pyroxenes: enstatite (orthopyroxene) and diopside (clinopyroxene). The addition of different mineral types to a rock generally decreases the overall anisotropy [Barruol \& Mainprice, 1993]. In pyroxenes, the fast direction orients normal to strain [Babuška, 1972] and therefore reduces bulk anisotropy in composition with olivine. Garnet is nearly isotropic and does not contribute to the seismic anisotropy of the bulk rock. Other minerals only occur in low percentage in the upper mantle, thus they are unlikely to contribute significantly to upper mantle seismic anisotropy. However, Dewandel et al. [2003] found reduced velocities in fast and slow directions for ser- 


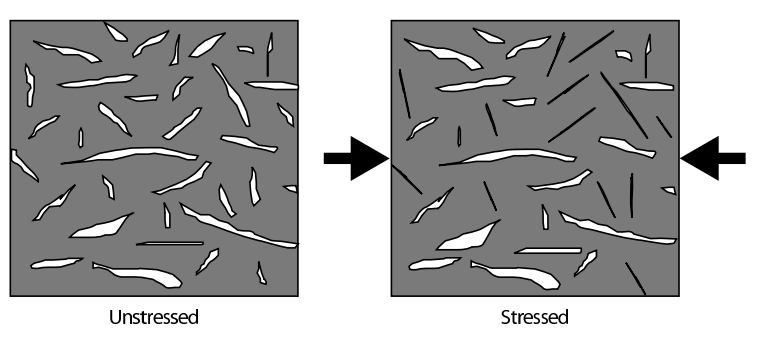

FIGURE $3.9 \begin{array}{r}\text { Shape- } \\ \text { preferred orientation }\end{array}$
Randomly oriented open
cracks appear isotropy (left).
In a stress regime (right),
cracks normal to the maxi-
mum stress orientation close,
while cracks parallel to it open
or remain open.

pentinised peridotite, so that overall percentage anisotropy increased from 6.2 to $8.6 \%$ for P-waves and from 4.6 to $6.1 \%$ for S-waves without changes in the fast orientation.

Deformation processes depend on temperature. For olivine, temperatures above $900^{\circ} \mathrm{C}$ are necessary to reorient the crystals [Estey \& Douglas, 1986]. If the temperature drops below this critical temperature during thermal relaxation, the anisotropy will be kept from the earlier, warmer tectonic processes as "frozen-in" the material [Silver \& Chan, 1991, 1988; Vinnik et al., 1992]. This means that earlier deformation history, such as the formation at mid-ocean ridges [Nishimura \& Forsyth, 1989; Blackman et al., 1996], can be preserved as fossil anisotropy in lithospheric structure. In terms of depth, in oceanic basins anisotropy is assumed to be frozen-in above $100 \mathrm{~km}$ and showing current deformation below, for continental regions the border is closer to $200 \mathrm{~km}$ [Savage, 1999]. However, these depths can vary broadly [Ritzwoller et al., 2004; Behn et al., 2004; Simons \& van der Hilst, 2003].

\subsubsection{Shape-Preferred Orientation (SPO)}

On a scale larger than the minerals, spatial inhomogeneities can yield anisotropy if they show a preferred alignment or layering [Karki et al., 2001]. Inclusions and cracks align perpendicular to minimum compressive stress orientations, as those perpendicular to maximum compressive stress close [Crampin, 1994] (figure 3.9). The fast polarisation is parallel to the cracks and therefore parallel to the maximum compressive stress [Crampin, 1984, 1994]. The anisotropic strength depends on the velocity contrast between the inclusion and the matrix. Strong contrasts produce high anisotropy.

Cracks are characteristic for the mid to upper crust. Crustal anisotropy has to be considered in mantle studies, as the crust under the receiver is always part of the travel path of the waves. But the crust is thin compared to the mantle and due to increasing pressure with depth, cracks start to close at about 10 to $15 \mathrm{~km}$ [Crampin, 1994]. Thus, contribution of crustal anisotropy to the overall teleseismic shear-wave splitting measurements is considered to be small. Worldwide averages of about $0.2 \mathrm{~s}$ (1.5-4\% S-wave anisotropy) are measured [Crampin, 1994; Silver, 1996]. Special types of SPO anisotropy are periodic thin layering and oriented melt pockets. Periodic thin layering (PTL) causes long-wavelength anisotropy due to fine layering of materials with contrasting velocities [Backus, 1962]. Hori- 
zontal layering, as predominant expected in the lower crust, causes anisotropy with vertical symmetry axis (vertical transverse isotropy). In this case, no azimuthal variations and therefore no splitting in vertical travelling shear-waves can occur. Oriented melt pockets (OMP) are effective in generating anisotropy due to a strong velocity contrast between the melt and the surrounding matrix [Kendall, 1994; Mainprice, 1997]. The anisotropic strength is very sensitive to melt fraction and to the shape of inclusions.

Some of the anisotropy causing structures can be on the order of the seismic waves (wavelength between $10^{1}-10^{5} \mathrm{~m}$ ). For these media, scaling becomes important and frequency dependent effects can occur. This type of anisotropy is called extrinsic, as it depends on the wavelength (e.g. SPO, PTL). Intrinsic anisotropy, in contrast, does not dependent on the wavelength of the seismic wave (LPO).

\subsection{Shear-wave splitting in subduction zones}

Numerous shear-wave splitting studies have been carried out in most subduction zones worldwide, presenting complex behaviour. Splitting parameters show a broad variety, with fast polarisations including both trench-parallel and trench-perpendicular $\Phi$ and delay times ranging from $0 \mathrm{~s}$ to greater than $3 \mathrm{~s}$ (table 3.2). Arguments for trench-parallel fast polarisations in oblique subduction environments and trench-perpendicular in extending backarc regions cannot be generalised, as counter-example show [e.g. Fischer \& Wiens, 1996].

Comprehensive overviews of shear-wave splitting measurements in subduction zones can be found in Buttles \& Olson [1998]; Savage [1999]; Wiens \& Smith [2003]; Kneller et al. [2008]. Moreover, databases covering worldwide anisotropy are developing and becoming available online, e.g. the "Upper Mantle Anisotropy Database" (ASU), the "Shear-wave splitting data base" (University of Montpellier) and the "Automated Splitting database" (ISC) .

TABLE 3.2 Shear-wave splitting in subduction zones

\begin{tabular}{|c|c|c|c|}
\hline Location & Local SWS & Teleseismic SWS & Reference \\
\hline $\begin{array}{c}\text { Honshu, Japan Sea } \\
\text { Southern Kurils } \\
\text { Mid Kurils } \\
\text { Japan } \\
\text { Kamchatka } \\
\text { Nazca } \\
\text { Columbia } \\
\text { Peru, central Chile } \\
\text { northern Chile } \\
\text { Alaska }\end{array}$ & $\begin{array}{c}\text { trench } \perp \text { (backarc), } \\
\text { trench } \|(\operatorname{arc}), \text { high } \delta t(1 \mathrm{~s}) \\
\text { trench } \perp \text { (backarc), } \\
\text { trench } \|(\operatorname{arc}), \text { high } \delta t(1 \mathrm{~s}) \\
\text { trench } \|, \text { high } \delta t \text { (up to } 0.8 \mathrm{~s}) \\
\text { trench } \| \\
\text { APM } \|, \text { high } \delta t(1 \mathrm{~s}) \\
\text { variable } \Phi, \\
\text { change from arc } \| \text { to arc } \perp \\
\text { variable } \Phi, \text { low } \delta t \\
\text { variable } \Phi, \text { small } \delta t(0.1 \mathrm{~s}) \\
\text { trench } \|, \text { up to } \delta t(0.4 \mathrm{~s}) \\
\text { trench } \| \\
\text { APM } \|, \sim 0.5 \mathrm{~s}\end{array}$ & $\begin{array}{c}\text { trench } \perp \\
\text { trench } \perp \\
\text { trench } \| \\
\text { trench } \| \\
\text { APM } \|, \delta t \sim 2 s \\
\text { APM } \|, \text { small } \delta t \\
\text { trench } \|, \text { low } \delta t \\
\text { trench } \|, \text { large } \delta t(3 \mathrm{~s}) \\
\text { APM } \|, \text { small } \delta t(<1 \mathrm{~s}) \\
\text { APM } \|,(1 \mathrm{~s}) \\
\text { trench } \|\end{array}$ & $\begin{array}{c}\text { Fischer \& Wiens [1996] } \\
\text { Fischer et al. [1998]; Smith et al. [2001] } \\
\text { Fouch \& Fischer [1996] } \\
\text { Fischer et al. [1998]; Hiramatsu et al. [1998] } \\
\text { Fouch \& Fischer [1996]; Fischer et al. [1998] } \\
\text { Fischer et al. [1998] } \\
\text { Fischer \& Yang [1994] } \\
\text { Sandvol \& Ni [1997]; Oda \& Shimizu [1997] } \\
\text { Nakajima \& Hasegawa [2004] } \\
\text { Fischer \& Yang [1994]; Peyton [2001] } \\
\text { Russo \& Silver [1994] } \\
\text { Wiens \& Smith [2003] } \\
\text { Polet et al. [2000] } \\
\text { Bock et al. [1998]; Polet et al. [2000] } \\
\text { Silver \& Chan [1991]; Mehl et al. [2003] }\end{array}$ \\
\hline
\end{tabular}


But shear-wave splitting not only varies between different settings; lateral variation across subduction zones are also observed. The arguably most outstanding include abrupt changes in fast polarisation over short distances across the arc region [Polet et al., 2000; Nakajima \& Hasegawa, 2004; Anderson et al., 2004].

The wide variability of shear-wave splitting observations in subduction zone settings diminishes the argument of coherent lithospheric mantle deformation and suggests the influence of local effects. The most common interpretations include mantle wedge corner flow [Buttles \& Olson, 1998], arc-parallel flow [Russo \& Silver, 1994], different olivine LPO types (e.g. B-type olivine in the cold mantle nose) [Kneller et al., 2005], the presence of melt [Holtzman et al., 2003a] and water [Karato \& Jung, 1998] in the mantle wedge, or any combination of them.

\subsection{Shear-wave splitting in North Island}

Several studies on seismic anisotropy were conducted on North Island over the last decade. Most of the studies are shear-wave splitting measurement from local and teleseismic events, predominantly recorded on southern and lower North Island (figure 3.10).

Gledhill [1991] was among the first to evaluate shear-wave splitting in southern North Island. Local events give large changes in splitting parameters between closely located stations around Wellington. This is interpreted to be caused by strong near-surface crack anisotropy with contributions from deeper, weaker anisotropy throughout the whole crust. Later, Gledhill \& Stuart [1996] and Gledhill \& Gubbins [1996] use both local and teleseismic events to constrain depth variations in seismic anisotropy. Local events yield anisotropy in the crust and the slab/mantle with nearly identical trench-parallel fast polarisations (40$50^{\circ}$ ). They determine anisotropic strengths of about $4 \%$ in the crust and very low values (about 1.4\%) in the slab. Teleseismic events yield fast polarisations of about $30^{\circ}$. The consistency of trench-parallel fast polarisations suggests similar deformation processes in crust, lithosphere and asthenosphere (internal coherent deformation) [Silver \& Chan, 1991]. Cracks cannot cause the crustal anisotropy, as fast polarisations do not fit with the dominant stress regime. However, fast polarisations match trends in the local geology, which is assumed to reflect present day deformation in the active tectonic regime. Multiple layers with similar fast polarisation, mainly in the slab and the subslab down to about $300 \mathrm{~km}$ are interpreted to add up to the observed high delay times.

Similar to the suggested model for southern North Island, Marson-Pidgeon et al. [1999] suggest coherent deformation occurring in the subslab asthenosphere and the mantle wedge caused by oblique subduction. Fossil anisotropy in the slab enhances the overall anisotropy. Brisbourne et al. [1999] present results from local shear-wave splitting from the lower North Island, south of the Volcanic Region. Differences in anisotropy are observed between the southern and northern stations. Stronger slab anisotropy in the south is interpreted as 


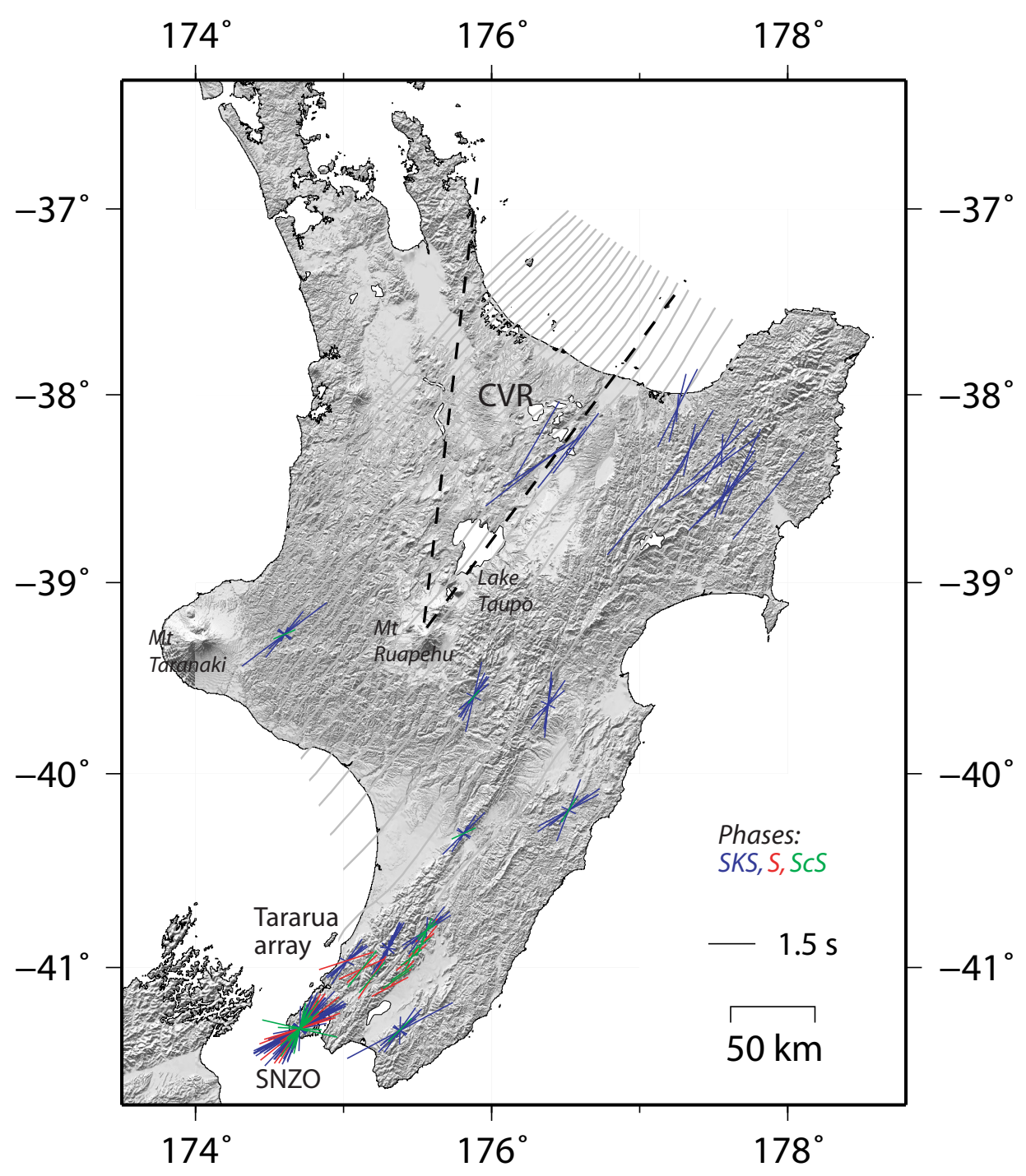

FIGURE 3.10 Summary of teleseismic shear-wave splitting in North Island. Single measurements from Gledhill \& Gubbins [1996]; Cochran [1999]; Brisbourne et al. [1999]; Marson-Pidgeon E Savage [1997]; Marson-Pidgeon et al. [1999]; Audoine et al. [2004]; Marson-Pidgeon \& Savage [2004]. Hatching (light grey) is slab isobaths.

caused by stronger slab coupling. The overall anisotropy is assumed to be $4 \%$ with trenchparallel fast polarisation down to about $210 \mathrm{~km}$. Matcham et al. [2000] suggest strong, trenchparallel anisotropy (4.4\%) in the slab with frequency dependent variations. First small-scale lateral variations in southern North Island were noticed in shear-wave splitting on S- and ScS-phases [Marson-Pidgeon \& Savage, 2004] across the Tararua array.

Recent work on central North Island is mainly based on local shear-wave splitting [Audoine et al., 2004; Morley et al., 2006]. In eastern central North Island, trench-parallel fast polarisations, as observed in southern North Island, prevail. However, there is a dramatic change from the prevailing trench-parallel fast polarisation out of the CVR towards trenchnormal fast polarisations within the CVR (figure 3.11). Vertical and lateral variations in the CVR are inferred [Audoine et al., 2004]. West of the CVR, delay times decrease and fast polar- 


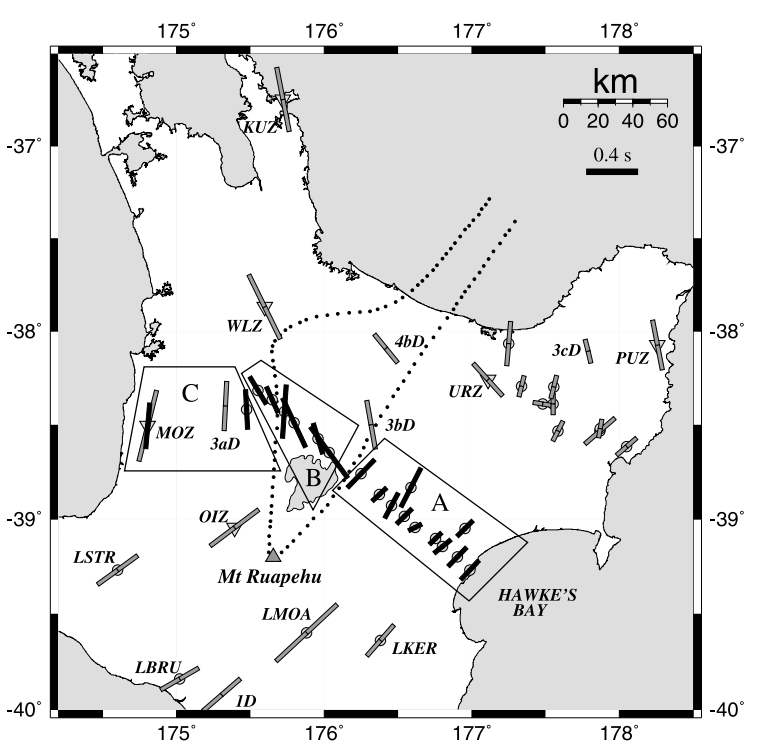

FIGURE 3.11 Local shearwave splitting results across central North Island from Morley et al. [2006] Observations are divided into groups with similar fast polarisation: A: trench-parallel (eastern North Island), B: $\Phi \sim 160^{\circ}$ (TVZ), C: North-South (western North Island).

isations are more scattered. A possible interpretation is the growing influence of the absolute plate motion on the observed fast polarisations [Morley et al., 2006]. Teleseismic measurements are rare across central North Island. Audoine et al. [2004] and Hofmann [2003] find consistent trench-parallel fast polarisation, with much larger delay times than observed in the south. However, those results are based on few measurements, and little attention was paid to the interpretation of the extreme delay time observations. The high average teleseismic delay times and the predominant thin crust caused by the extensional regime in central North Island lead to the conclusion of a strong asthenospheric contribution [Duclos et al., 2005]. The only direct measurements of seismic anisotropy in North Island from a xenolith [Duclos et al., 2005], however, only exhibits small anisotropy of about 3.5\% (figure 3.5). To understand the influence of varying seismic anisotropy across the Hikurangi subduction zone, waveform modelling has been suggested [Audoine et al., 2004; Marson-Pidgeon \& Savage, 2004], but not conducted until now (cf. chapter 6). 


\section{Chapter 4}

\section{Data}

\subsection{Deployments}

The waveforms used in this study originate from different seismic stations that consist of various instruments and were deployed over different time frames. The main data set is derived from the Central North Island Passive Seismic Experiment (CNIPSE), a collaboration between Leeds University, Institute of Geological and Nuclear Sciences and Victoria University of Wellington. The deployment was extended twice after the main operation time. Those deployments will be called CNIPSE2 and WCNIPSE in the following. Additional data used in this study comes from a temporary deployment in Northland (NORD) and from stations of the GeoNet national network. This chapter describes details about those different data sets. Figure 4.1 gives an overview map with all stations; detailed information is compiled in table B.1.

\subsubsection{CNIPSE}

The Central North Island Passive Seismic Experiment (CNIPSE) was conducted in 2001. It comprised a total of 74 stations, out of which 32 were broadband stations. The experiment was a collaboration of Institute of Geological and Nuclear Sciences (IGNS), University of Leeds (UK) and Victoria University of Wellington (VUW). The deployment started in January 2001 and ended in June 2001. The westernmost instrument (YUPC) was kept in place for a further six months (July to December 2001) as part of the CNIPSE2 deployment (cf. next section). A detailed description of CNIPSE can be found in Reyners \& Stuart [2002]. Eighteen of the broadband stations were deployed along a line approximately perpendicular to the trench of the Hikurangi margin crossing most of the subduction arc system on North Island. The line starts at the east coast and ends about $80 \mathrm{~km}$ away from the west coast. These stations building the CNIPSE line are the ones considered in this study (red circles in figure 4.1). Typical station spacing along the line is about $10 \mathrm{~km}$. The location of the line is chosen close to the refraction/reflection line from the coinciding NIGHT project [Henrys 
FIGURE 4.1 Station distribution across North Island. Colours indicate the different deployments: CNIPSE (red) with Leeds stations (dark red), WCNIPSE (orange), NORD (cyan), GeoNet (yellow) other stations (pink).

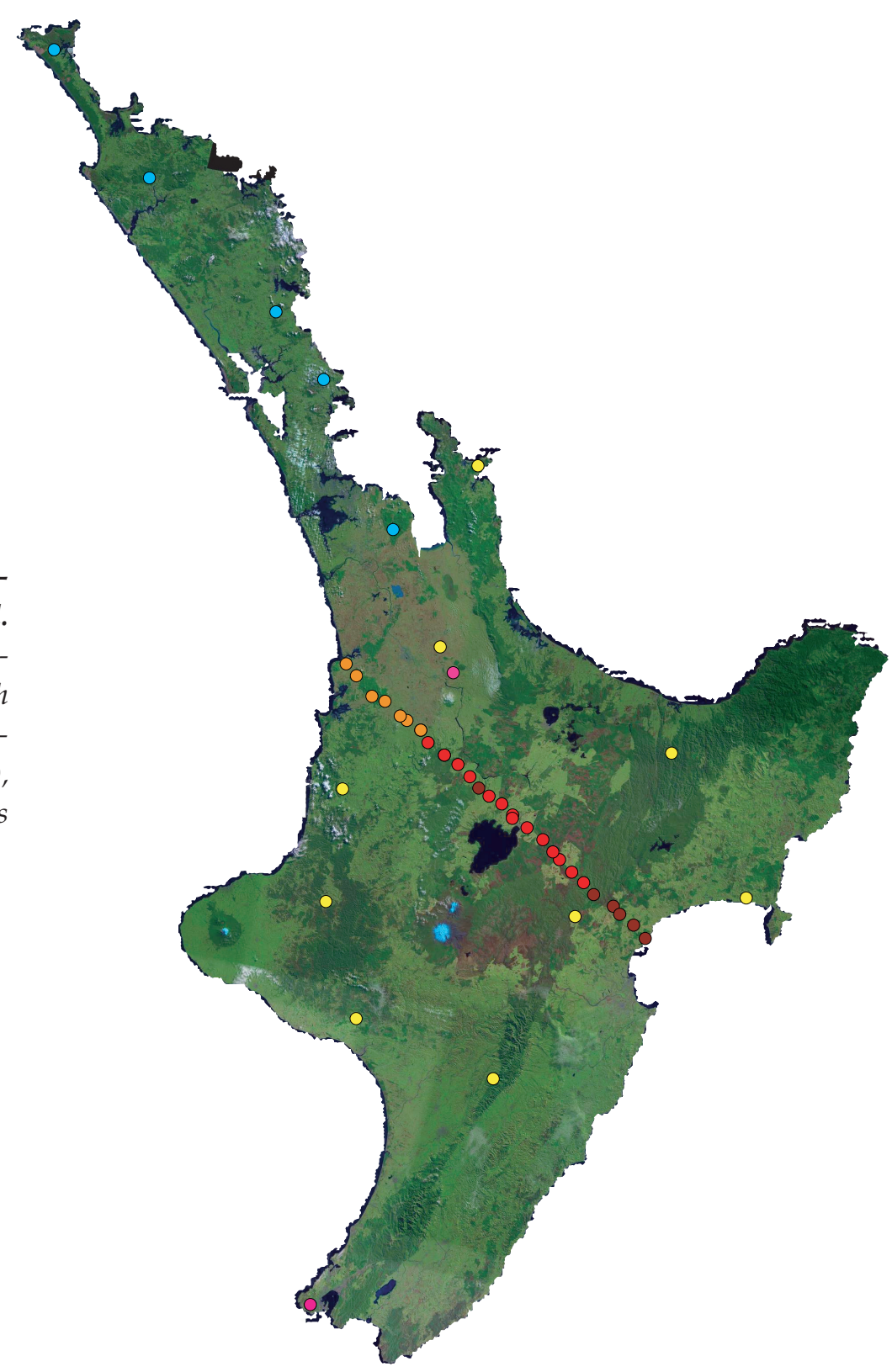

\& the NIGHT working group, 2001], in order to compare results. Sampling rates for all broadband stations are $100 \mathrm{~Hz}$.

\subsubsection{CNIPSE2}

After the end of the CNIPSE deployment, in July 2001, two of the three VUW broadband stations (WAIC and SCHC) were moved and redeployed northwest of the remaining station YUPC. This western extension of the CNIPSE line, in the following called CNIPSE2, stayed deployed for the rest of the year and was taken out in December 2001. The sampling rate was decreased from the original $100 \mathrm{~Hz}$ to $50 \mathrm{~Hz}$. Further details about the deployment can be found in Hofmann [2003]. 

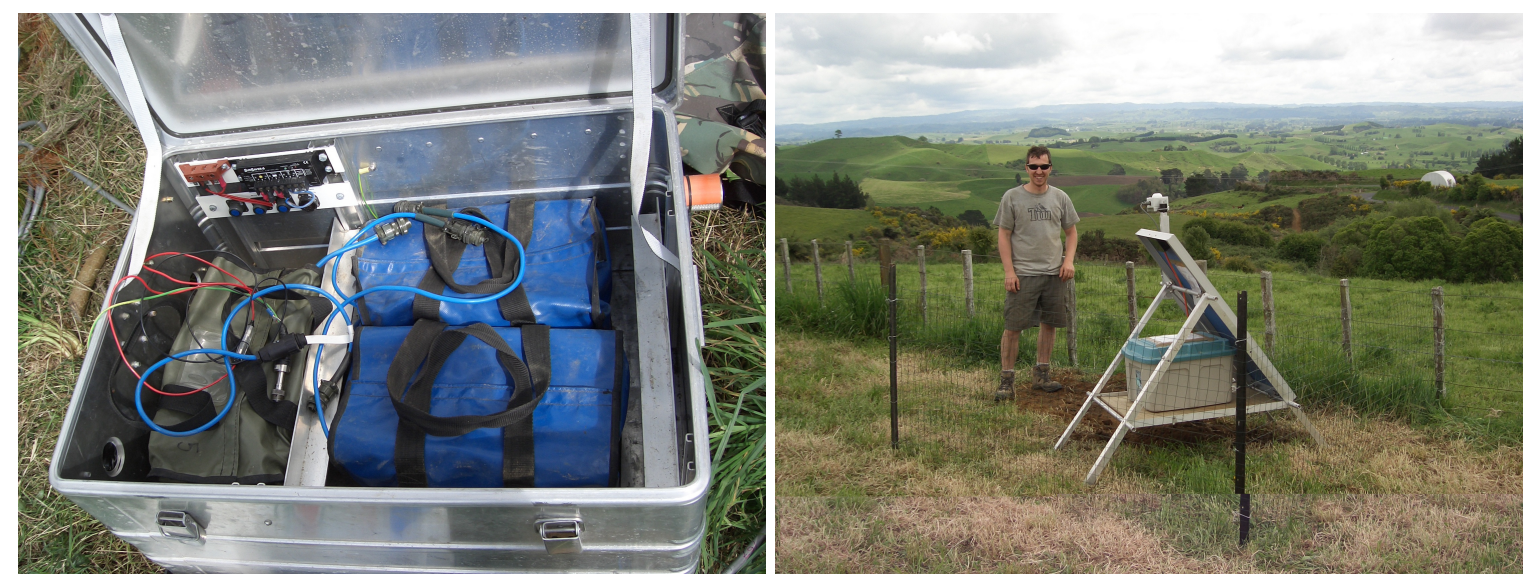

FIGURE 4.2 Station set up for broadband recorder. Left: The box for the digitiser containing three $12 \mathrm{~V}$ batteries. Right: The fenced station with solar panel and GPS unit.

\subsubsection{WCNIPSE}

With the CNIPSE2 extension, the transect still did not reach the west coast of the North Island. Therefore, WCNIPSE, the Western extension of the CNIPSE line, was conducted at the end of 2004 with the aim to complete the line of broadband recorders across North Island. The temporary deployment consisted of seven broadband three-component recorders (orange circles in figure 4.1). The easternmost station of the line reoccupies the location of WULC, the westernmost station of CNIPSE2. The last, westernmost station is located close to Raglan, on the west coast. The nominal station spacing of the WCNIPSE line is $10 \mathrm{~km}$ and the sampling rate is set to $50 \mathrm{~Hz}$.

All station locations were on farmland, away from dominant noise sources. Sites were chosen to be close to bedrock and the sensors were buried about $20 \mathrm{~cm}$ deep in order to reduce noise and achieve good ground coupling. All stations had solar panels as their main energy source attached to two to four $12 \mathrm{~V}$ batteries. Data were recorded continuously and stored on $2 \mathrm{~Gb}$ hard drives for the ORION recorders and on $4 \mathrm{~Gb}$ microdrives for the REF TEK recorders. The stations were serviced and data downloaded every six to eight weeks. Orientations were checked again when sites were picked up. All stations were set up with a GPS unit for correct timing and positioning. A surrounding fence protected stations against cattle (figure 4.2).

Interferences with other projects required a temporary stop for two instruments (STEC, YTFC) of about six weeks and to dismantle three stations (PUKC, VERC, PEAC) earlier than the others ( $c f$. table B.1).

Station problems included low battery power at several sites despite solar panels and some disruptions of the GPS. Station YTFC did not record any data in the period from 27. March 2005 to 4. July 2005 due to a disk problem. Noise was a problem at stations STEC due to woodworks before 3. July 2005, at station FLYC, where a pump was noticed later in 
the deployment (26. March 2005) and some MX racing took place. Stations VERC and PEAC were located close to running water that might have increased during autumn.

\subsubsection{NORD}

The NORthland Deployment (NORD) was a temporary deployment in Northland, the northernmost part of New Zealand. The deployment started in August 2002 with five broadband seismic stations and ended in February 2004, when three stations were taken out (MKAZ, MATA, TIKO). Two stations - OUZ and WCZ - were later converted into national network stations (cf. section 4.1.5). The stations were distributed along the length of Northland with spacings of about 50 to $100 \mathrm{~km}$. Sampling rates for all stations were $50 \mathrm{~Hz}$. Further details about the deployment can be found in Duclos [2005]. Data from the NORD deployment has been used for SKS splitting measurements [Duclos, 2005] and a joint receiver function, surface wave analysis [Horspool et al., 2006].

\subsubsection{GeoNet}

The New Zealand National Seismograph Network provides data collection capability on short- and broadband stations throughout the country. Stations consist of Güralp (CMG3ESP) or Streckheisen (STS-2) broadband seismometer and Quanterra Q4126 or Q330 six channel 24 bit data logger. The GeoNet project allows direct access of SEED data files with AutoDRM via the internet (www.geonet.org.nz).

Data from eight national network broadband stations surrounding the CNIPSE line (WAZ, TSZ, BKZ, URZ,VRZ, HIZ,TOZ and KUZ), extend the dataset used in this study.

\subsection{Processing}

This section provides an overview over the process of data handling starting from the raw data collected in the field to the actual data set used for the various analyses in this work. The first section describes the preprocessing applied to the raw data. The second section describes the process of instrument corrections. These corrections have to be applied as data used in this study originates from different types of instruments.

\subsubsection{Preprocessing of passive seismic data}

Seismic data for this study have been recorded using two different kinds of digitisers - Orion and REF TEK. Preprocessing of the raw data differs for both types of digitisers, until data is converted into a common format (SAC - seismic analysing code). 
TABLE 4.1 Event block structure

\begin{tabular}{|c|c|c|c|c|}
\hline Block & Magnitude & $\begin{array}{c}\text { Distance } \\
\left({ }^{\circ}\right)\end{array}$ & $\begin{array}{c}\text { Cut time } \\
\text { before arrival (s) }\end{array}$ & $\begin{array}{c}\text { Cutting length } \\
\text { (s) }\end{array}$ \\
\hline \hline 1 & $2-2.9$ & 1.5 & 60 & 180 \\
2 & $3-3.4$ & 3 & 60 & 300 \\
3 & $3.5-4.4$ & 5 & 60 & 480 \\
4 & $4.5-4.7$ & 18 & 60 & 900 \\
5 & $4.8-4.9$ & 30 & 60 & 900 \\
9 & $5-5.9$ & 100 & 300 & 1500 \\
10 & $6-6.9$ & 180 & 300 & 3600 \\
11 & $7-10$ & 180 & 300 & 7200 \\
\hline
\end{tabular}

The Orion data recorder stores data on $2 \mathrm{~Gb}$ data discs. After loading data from those discs onto a computer system, they need to be decompressed to their actual size. The downloads for all the stations should be gathered in the same experiment directory. They are then run through a standard Orion processing routine to create standard event files in SACformat (for details see appendix C.1). Those are combined in SEED-format files containing all records for one earthquake at all the recording stations. A manual for preprocessing Orion raw data with all the processing details was assembled during the work on this project ( $c f$. supplement CD-ROM). Selection of recorded events is based on earthquake event lists, which are retrieved from GeoNet for local events, from IRIS for recent teleseismic events and, because of revised locations and timing, from ISC for older teleseismic events. Not all events get extracted. Instead, a selection system is applied choosing events depending on magnitude and distance. Events of the same magnitude/distance relation build a "block" (table 4.1). From the SEED files, data can be extracted into different formats. Here SAC format is used.

REF TEK data get downloaded directly onto a UNIX system, into a folder named after the REF TEK recorder number. Similar to the Orion, a processing sequence needs to be applied, here based on software provided by Nanometrics and Passcal (for details see appendix C.2). However, the software package available at VUW does not allow creating SEED files. Therefore, the result of the REF TEK processing is data in m(ini)seed- or SAC-format. The disadvantage of miniseed is their limited header information and lack of instrument response information. Event selection is based on the same catalogue data and the same block structure (table 4.1) as for the Orion processing. A manual for preprocessing REF TEK raw data was also assembled during the work on this project ( $c f$. supplement CD-ROM).

\subsubsection{Instrument correction}

Correcting for instrument response is especially important when working with amplitudes, waveforms or spectra recorded from different kinds of instrument, i.e. digitisers and sensors. The influence of the digitisers on the distortion of the waveform is less than from the sensors. The biggest issue is the difference in corner frequencies for the seismometer (fig- 


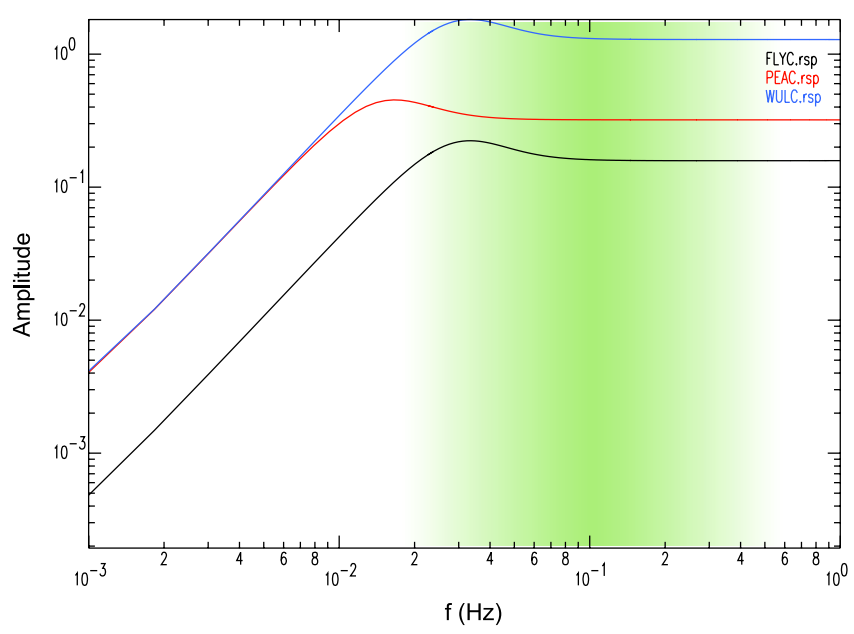

FIGURE 4.3 Amplitude response for three different stations (FLYC, PEAC, WULC) as a function of frequency, illustrating differences in corner frequencies and gains. The shaded area indicates the main frequency range considered in this study.

ure 4.3). The application of an instrument response correction flattens the response spectra of the seismometers, so that waveforms and amplitudes are more similar after the correction. The WCNIPSE deployment used two types of broadband seismometer (Güralp CMG-40T and Güralp CMG-3T) and short period seismometers (Marks Products L4C-3D) in conjunction with two kinds of digitisers (Orion and REF TEK).

\section{Theory}

The instrument response of any instrument describes the filtering process affecting the raw, original waveforms during recording with the seismometer and sampling with the digitiser. The recorded waveform spectra $S(f)$ is a product of the far-field source spectra $A(f)$, the site response $R(f)$, the attenuation spectra $B(f)$ and the instrument response $I(f)$ :

$$
S(f)=A(f) * I(f) * R(f) * B(f)
$$

Therefore, correcting for instrument responses removes the influence of those filters, which can be done through deconvolution. Filters are described by their poles and zeros. The effect of the gain is taken into account by an amplification factor. These values are instrument specific and should be supplied with the purchase of the instrument.

\section{Practical application}

The standard processing for raw Orion data (appendix C.1) already contains a step to include all important information for instrument corrections. The response information gets put into the SEED files and can be extracted together with the data. The correction can then be applied directly using the "transfer" function in the SAC software. Therefore, the best way to achieve a good instrument correction to ensure a precise preprocessing. However, the standard processing only works for the Orion digitisers, as stages from three onwards are describing the digital filters (FIR filter) in the digitisers. It is possible to alter these values 


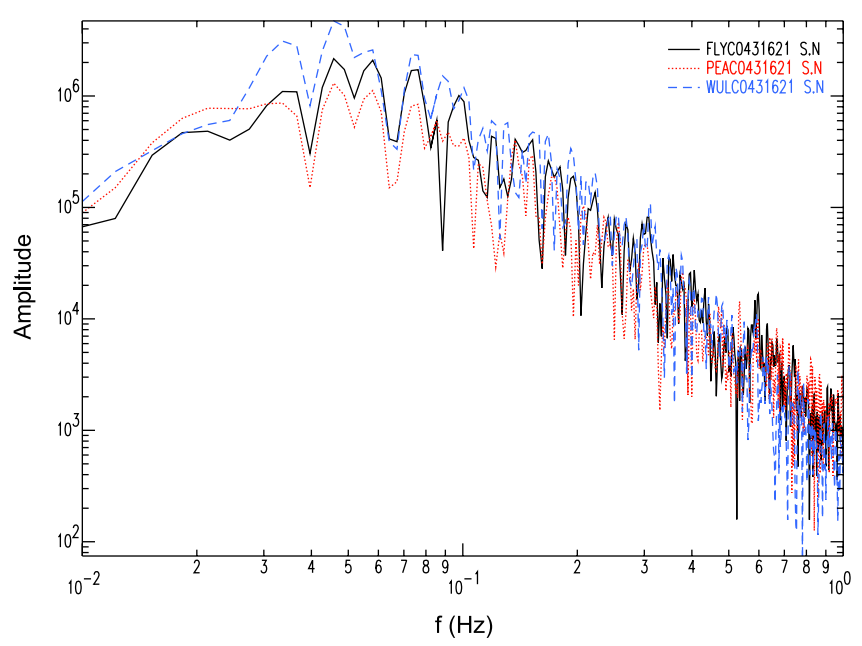

(a) Raw data

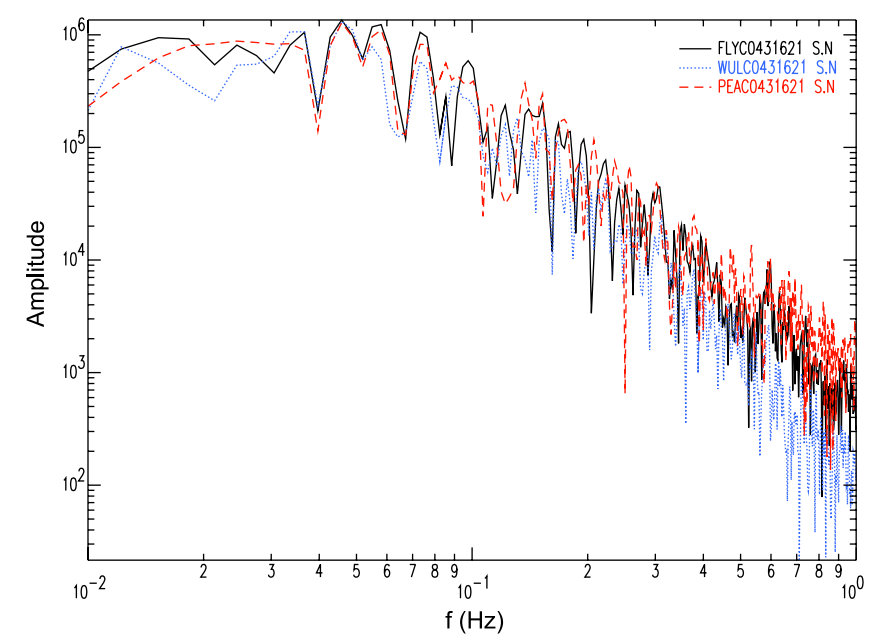

(b) Instrument response corrected using response file

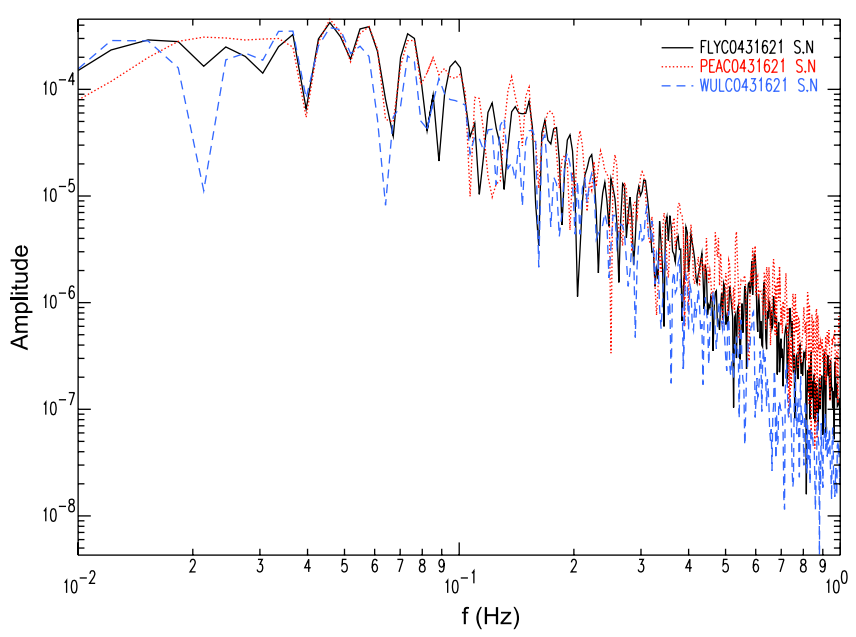

(c) Instrument response corrected using poles and zeros

FIGURE 4.4 Raw and corrected amplitude spectra for the same event recorded at three different stations (FLYC, PEAC, WULC) comparing raw data with two different methods of instrument correction. 


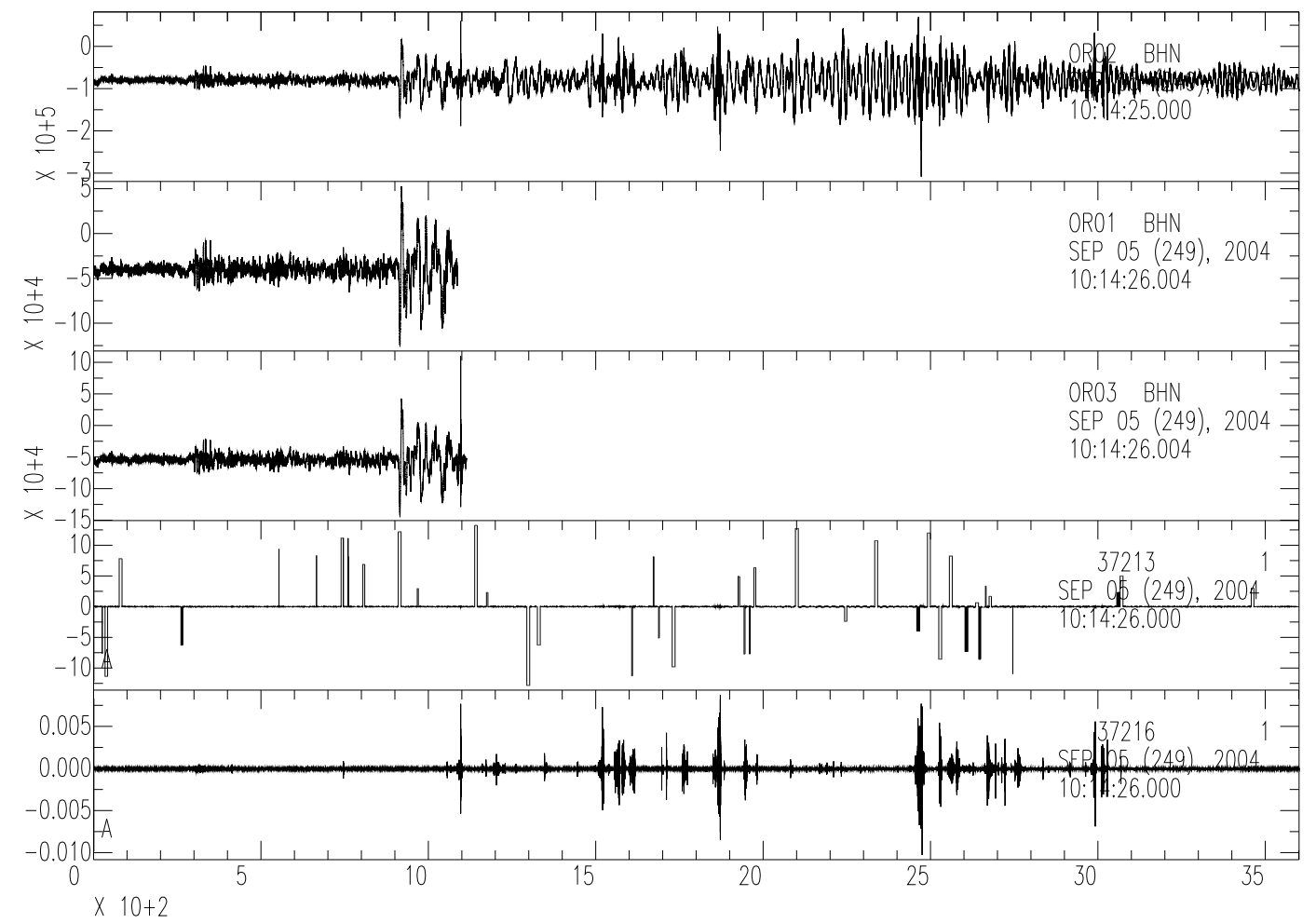

FIGURE 4.5 Comparison of cluster test recordings. The units from top to bottom are: Orion o162 with Güralp CMG-40T T4433, Orion o177 with Güralp CMG-40T T4430, Orion o178 with Güralp CMG-40T T4432, REF TEK 915D with Güralp CMG-40T T4431 and REF TEK 9160 with Marks Products L4C-3D L656. Problems with the two REF TEK recordings are obvious in the spiky nature of the traces.

for different digitisers but it is complicated (details are given in the SEED manual [Iris Consortium, 1993]).

For REF TEK digitisers, VUW does not have the software to create full SEED files. Thus, Segy- or m(ini)SEED-format data with limited header details and no instrument response information are created from the raw data. The instrument response is then described using the poles and zeros of the sensor and a sensitivity. The later can be calculated by multiplication of the gains/sensitivities of stages one (sensor), two (amplifier) and three (recorder). In the easiest case, that would be:

$$
\operatorname{gain} 1[V / m / s] * \operatorname{gain} 2[] * \text { gain } 3[\text { counts } / V]=\operatorname{gain}[1 / \mathrm{m} / \mathrm{s}]
$$

Comparisons show only minor differences between the two methods (figures 4.5(b) and 4.5(c)). The effect of the instrument correction becomes evident in the closer resemblance of the raw (figure 4.5(a)) and corrected spectra (figures 4.5(b) and 4.5(c)). The fit for the spectra is especially good in the lower frequencies, the frequencies considered in teleseismic studies. The absolute amplitude scales differ because SAC scales the output of response corrections to nanometre, but the output of other corrections, like pole-zero files, to metres.

Applying the corrections to data from a cluster- or huddletest helps to ensure that the 
removal of the instrument response is working properly. For a clustertest, all relevant instruments are deployed in the same location, next to each other, for a short time, usually a few days. It is assumed that the far field source spectra $A(f)$, the site response $R(f)$ and the attenuation spectra $A(f)$ are the same for all the recorded waveforms from a far earthquake recorded at the array. The only remaining difference between the waveforms is then caused by the instrument response $I(f)$ (equation 4.1). After removal of the instrument response, the recorded waveforms and their spectra should be nearly identical.

A clustertest was conducted before the start of the WCNIPSE deployment, using the following sensor-digitiser configurations: four Marks Products L4C-3D seismometers with REF TEK 130 digitisers, three Güralp CMG-40T seismometers with Orion digitisers and one Güralp CMG-40T seismometer with a REF TEK 130 digitiser.

Unfortunately, at the time of the clustertest, not all instruments later deployed in the field were available and all but one REF TEK digitisers were paired with the shortband L4C3D seismometers. The one connected to the broadband seismometer had problems recording (figure 4.5), and so did another REF TEK recorder (bottom trace in figure 4.5). Therefore, the usable data for the purposes of this study only include three Orions paired with CMG$40 \mathrm{~T}$ broadband sensors. In this case, instrument corrections are not applicable. 


\section{Chapter 5}

\section{Shear-wave splitting across central North Island ${ }^{1}$}

\subsection{Abstract}

Shear-wave splitting measurements from SKS-, SKKS-, S- and ScS-phases reveal the anisotropic structure of North Island, New Zealand. Waveforms were recorded on temporary and permanent broadband seismographs. Strong anisotropy varies rapidly across the Hikurangi subduction zone. These changes constrain the width of the deformation associated with the plate boundary and define three different regions of mantle flow. Stations in the forearc region, eastern central North Island record fast polarisations subparallel to the trench and moderate delay times of around $2.5 \mathrm{~s}$, consistent with earlier studies of North Island. This is interpreted as caused by trench-parallel mantle flow beneath the subducted slab with a possible contribution of trench-parallel fossil anisotropy within the subducted slab. Delay times increase towards the extending Central Volcanic Region (CVR) with values up to $4.5 \mathrm{~s}$, one of the largest delay times measured worldwide. Strong S-wave anisotropy $(7.5 \%)$ through $80 \mathrm{~km}$ in the mantle wedge in addition to the sub-slab anisotropy can explain the observed high delay times. One mechanism to create such high anisotropy is melt segregation of partially molten rock. A frequency dependence of this mechanism could also explain persisting discrepancies between local and teleseismic shear-wave splitting measurements. To the west, delay times decrease systematically. Measurements in the far backarc region of western central North Island show no apparent splitting. This suggests that mantle wedge dynamics terminate beneath the western border of the CVR. The apparent isotropy beneath the far backarc may be related to local small-scale mantle convection or vertical mantle flow, possibly caused by the detachment of lithosphere.

${ }^{1}$ accepted for publication in Tectonophysics [Greve et al., 2008] 


\subsection{Introduction}

Seismic anisotropy, the dependence of seismic wave speed on polarisation, is a measurable property of the crust and upper mantle that is related to stress or strain orientations. Therefore, it is a useful tool with which to study the complex dynamic processes at subduction zones.

In the upper $10-15 \mathrm{~km}$ of the crust, anisotropy is caused by shape-preferred orientation (SPO) of cracks, fractures and inclusions aligned with strain due to the current stress regime [Crampin, 1994]. In contrast, deeper anisotropy is often ascribed to the latticepreferred orientation (LPO) of olivine crystals due to past deformation "frozen" in the lithosphere or to current strain orientations in the mantle related to asthenospheric flow [e.g. review by Savage, 1999]. For simple shear deformation mechanisms under dry conditions the fast axis (a-axis) of olivine orients parallel to the extension direction, or shear in the infinite strain limit [Ribe, 1989]. Recent studies [Jung et al., 2006] reveal a more complicated relationship between deformation and mantle flow under different pressure conditions and the influence of water. In the mantle wedge, the abundance of melt can cause anisotropy due to SPO of melt filled inclusions or the layering of segregated melt bands [Holtzman et al., 2003b].

A shear-wave travelling through an anisotropic medium splits into two components propagating with different speeds. The two waves are polarised nearly orthogonal to each other with the polarisation of the faster travelling component commonly called the fast polarisation or fast direction. The delay time separating the two components after exiting the medium provides a measure of the length of the travel path through the anisotropic medium and the difference in wave speeds in the media.

Shear-wave splitting of teleseismic phases is a well-established tool to study subduction related processes because it relates seismic observations to deformation [e.g. Ando et al., 1983; Babuška \& Cara, 1991; Savage, 1999; Silver \& Chan, 1988]. Measurements [e.g. Nakajima \& Hasegawa, 2004] and modelling [e.g. Hall et al., 2000; Lassak et al., 2006; Buttles \& Olson, 1998] show that across subduction zones, anisotropy can change over short distances.

North Island, New Zealand, located along Hikurangi subduction zone, the southernmost extension of Tonga-Kermadec arc, provides an opportunity to study a subduction zone from on-shore stations (figure 5.1). Most of the teleseismic shear-wave splitting studies from North Island focus on its southern part, with several portable studies, and IRIS network station SNZO delivering high quality broadband data since 1992 (figure 5.2) [Gledhill \& Gubbins, 1996; Marson-Pidgeon \& Savage, 1997, 2004; Marson-Pidgeon et al., 1999; Matcham et al., 2000]. Southern North Island is located in the forearc region, and consistent fast polarisations subparallel to the trench are inferred to be caused by trench-parallel mantle flow below the slab. Results from local earthquakes [Audoine et al., 2000; Brisbourne et al., 1999] 


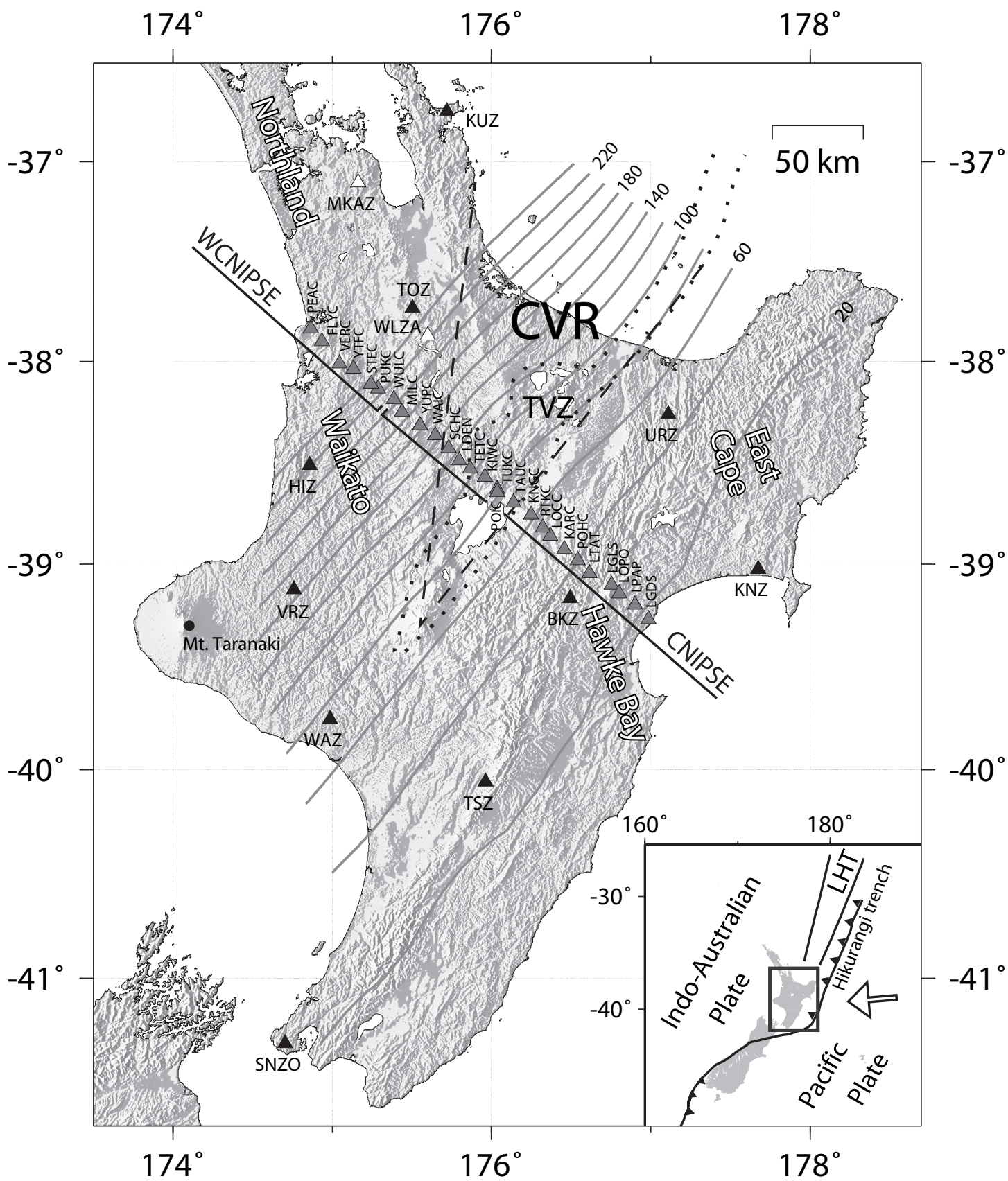

FIGURE 5.1 Overview map. Topographic map of central North Island with locations of broadband stations used in this study marked as triangles. Three-letter codes name permanent GeoNet stations (black triangles) and four-letter codes indicate temporary stations ((W)CNIPSE stations as grey triangles, other temporary deployments as white triangles). The dashed line marks the boundary of the Central Volcanic Region (CVR) and the dotted line the boundary of the Taupo Volcanic Zone (TVZ). Contours indicate the depth to the top of the subducted slab as determined by earthquake hypocenters. Inset shows the location of the study area with plate boundary (solid line); arrow represents the absolute plate motion vector. LHT: Lau-Havre trough. 
are more variable. Until recently, only a few shear-wave splitting studies were carried out on the northern part of the forearc (Hawke Bay) or behind the forearc in central North Island [Audoine et al., 2004; Klosko et al., 1999].

Northwest-southeast extension of $8-15 \mathrm{~mm} / \mathrm{yr}$ [Wallace et al., 2004] has formed a wedgeshaped region of thinned crust in the Central Volcanic Region (CVR), the backarc region of central North Island. It marks the southern end of the Lau-Havre trough and is therefore a region of transition between oceanic and continental rifting. Characteristics of the CVR are high heat output, shallow crustal seismicity and negative residual gravity anomalies [Stern et al., 2006]. Active silicic volcanism takes place in the Taupo volcanic zone (TVZ), along the eastern half of the CVR [Cole, 1990]. Audoine et al. [2004] relate trench-parallel fast polarisations from teleseismic phases to either deep anisotropy down to $660 \mathrm{~km}$ due to trenchparallel mantle flow or shallower, unusually high anisotropy $(6 \%)$ due to the presence of water-rich olivine in the mantle wedge. In the far backarc region of Waikato, teleseismic shear-wave splitting measurements are lacking. In contrast to the CVR, the most recent volcanism in the region is Miocene [Briggs, 1986]. One exception to this pattern is the presently active Mt. Taranaki southwest of the CVR (figure 5.1). Under the west coast, deep earthquakes mark the slab down to around $250 \mathrm{~km}$ [Anderson \& Webb, 1994; Ansell \& Bannister, 1996].

With an increasing number of national network stations [GeoNet, 2001], it is now easy and fast to analyse splitting all across New Zealand. A comprehensive shear-wave splitting analysis using GeoNet stations all over North Island [Duclos et al., 2005] confirmed previous results [Audoine et al., 2004]. But an interesting variation was observed at one station (TOZ) in northwestern central North Island (figure 5.2). For this station no splitting was observed, with one exception showing a fast polarisation almost perpendicular to the trench. Because of the high attenuation, no GeoNet station provided S-arrivals good enough to yield splitting measurements in the CVR.

The central North Island Passive Seismic Experiment (CNIPSE) [Reyners \& Stuart, 2002] in 2001 aimed to gather more information on subduction processes (figure 5.1). Two teleseismic events yielded different splitting parameters, which were interpreted as backazimuthal dependency caused by two layers, one in the upper mantle and another in the lower mantle or the D“ layer [Hofmann, 2003]. Local splitting analyses across the CNIPSE line [Morley et al., 2006] yielded maximum delay times of $0.35 \mathrm{~s}$ interpreted as caused by anisotropic regions with maximum depths increasing from $60-115 \mathrm{~km}$ from east to west. Fast polarisation show a dramatic change from trench-parallel in eastern North Island to trench-perpendicular in the CVR. This is interpreted as a change from crustal anisotropy in the east to uppermost mantle anisotropy due to trench-normal mantle flow in the mantle wedge under the CVR. For western North Island, local splitting fast polarisation change to north-south [Morley et al., 2006], consistent with the absolute plate motion, but data are sparse, as the line initially did not extend to the west coast. This gap in the CNIPSE line was completed in November 2004 when seven broadband seismometers were deployed in the 


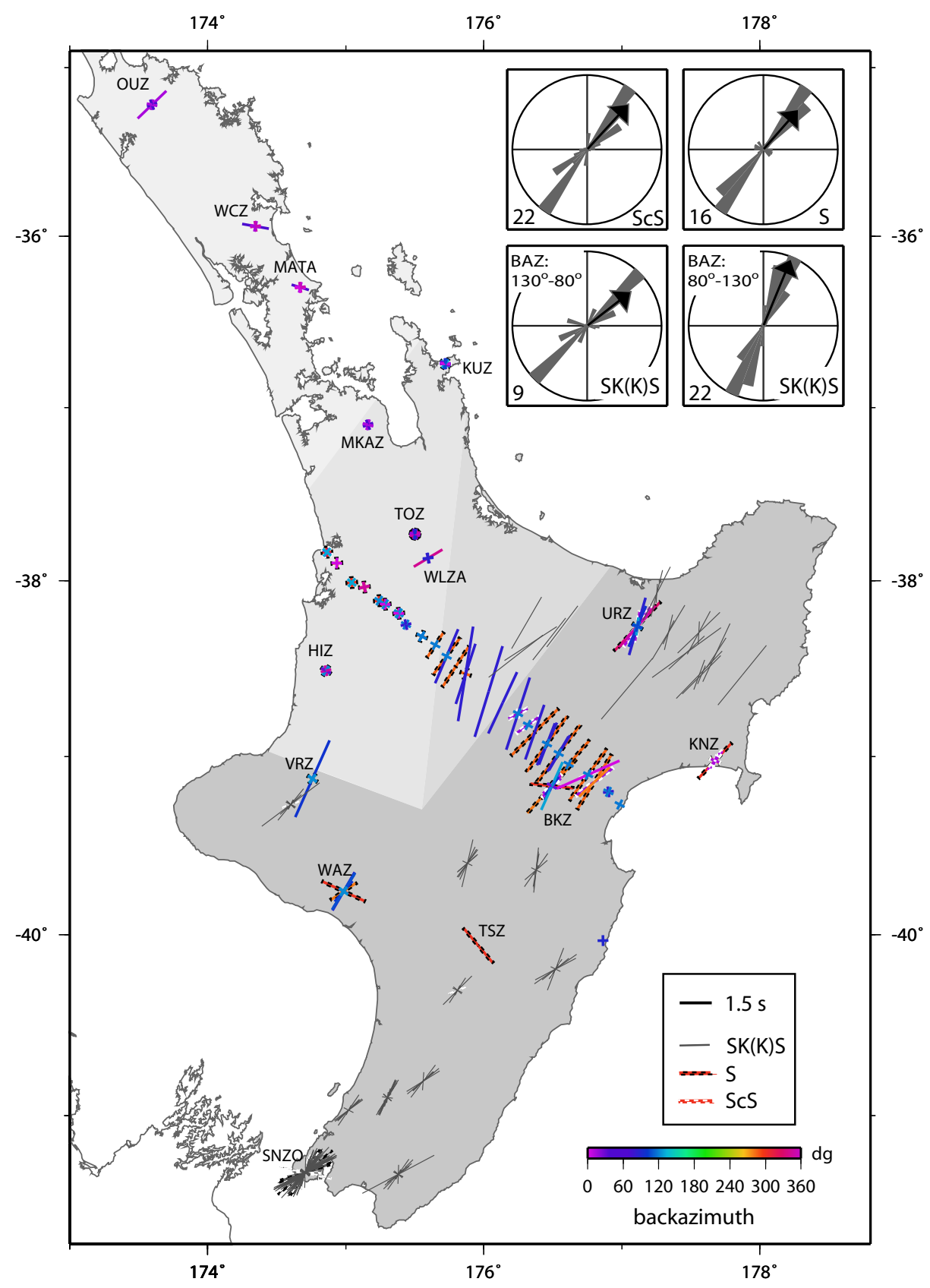

FIGURE 5.2 Map with splitting measurements. New splitting measurements (coloured) combined with all individual splitting measurements available to date (grey). Orientation of each line is parallel to measured fast polarisation; length is scaled according to delay time. Each line is plotted at the location of the recording station and represents a single measurement. New measurements are coloured according to the backazimuth of the measured phase. Plain lines indicate SK(K)S results, black dotted line ScS and white dotted lines S (dots on top of colour code). The histograms show fast polarisations from ScS (top left), $S$ (top right) and from SK(K)Sphases for backazimuths $130^{\circ}<B A Z<80^{\circ}$ (bottom left) and $80^{\circ}<B A Z<130^{\circ}$ (bottom right) for results from this study. The numbers in the lower left corner of each histogram represent the number of events used in the histogram and the arrows indicate mean polarisations. Suggested anisotropic regions for central North Island are coloured in different shades of grey (light=Waikato and Northland, medium $=C V R$, dark=Hawke Bay and rest of North Island). 
Waikato during the WCNIPSE experiment.

In this study we present new splitting measurements determined for the temporary WCNIPSE stations and for eight permanent GeoNet stations on central North Island and improved shear-wave splitting measurements from the temporary CNIPSE deployment (figure 5.1). There are strong variations of splitting parameters in North Island, with high delay times in the TVZ and no apparent splitting across western central North Island (section 5.4). These observations define different anisotropic regions across the subduction zone (section 5.5.1). Section 5.5.2 discusses possible causes of the observed anisotropy in those regions in the context of processes in the mantle wedge and changes in mantle flow across the subduction zone (section 5.5).

\subsection{Method and data: Automated shear-wave splitting measure- ments}

To study mantle anisotropy, core-refracted phases such as SKS, SKKS or PKS are usually used, as their steep incidence angles provide good lateral resolution. They respond only to anisotropic material located between the outer core and the recording station. Additional information is needed to confine the location of the anisotropy further. In this study we use SK $(\mathrm{K}) \mathrm{S}$, teleseismic S- and regional ScS-phases for splitting measurements. In contrast to SK(K)S-phases, the anisotropy detected by S- and ScS-phases could lie anywhere on their travel path and source side anisotropy can contaminate results. To constrain this uncertainty we only use deep events, following the generally accepted idea that most anisotropy is concentrated in the upper mantle [Fischer \& Wiens, 1996; Savage, 1999; Silver \& Chan, 1991]. Even if source side anisotropy is present, changes observed for a single event across a closely spaced network must be related to receiver side structures. The shallower incidence angles, especially for S-phases, can provide information to overcome the lack of vertical resolution of core-refracted waves and thus help to resolve the depth of the anisotropic medium. Due to higher frequency content, regional ScS-phases have narrower Fresnel zones and can potentially be sensitive to different anisotropic regions than $S$ and SK(K)S phases. Generally, it is assumed that higher frequencies are more sensitive to shallower anisotropy [e.g. Rümpker et al., 1999; Schulte-Pelkum \& Blackman, 2003]. Therefore, shear-wave splitting measurements on local S-phases provide further depth constraints. 

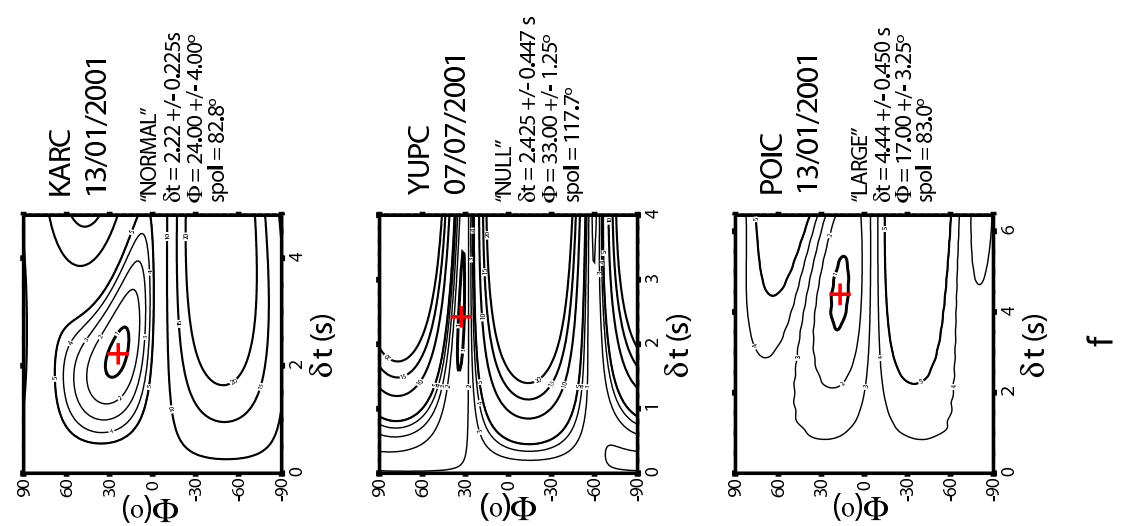

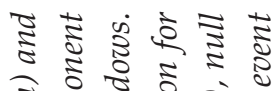

ह ㅎำ

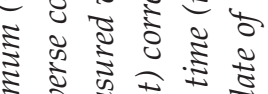

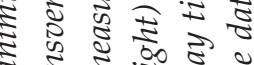

₹

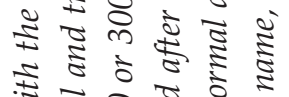

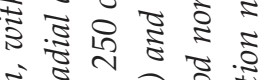

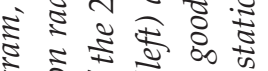
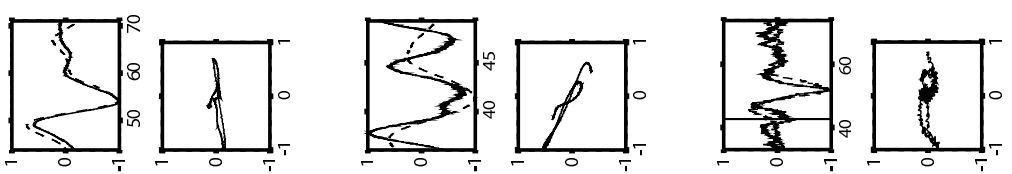

की

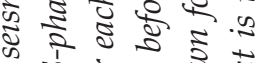

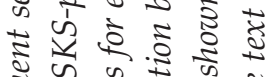
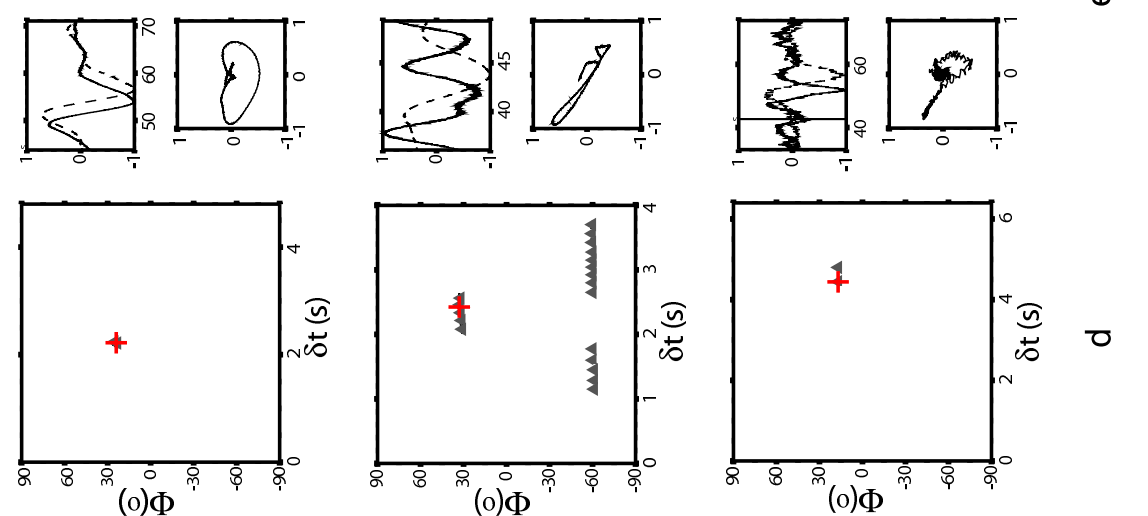

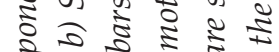

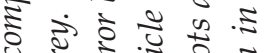

के

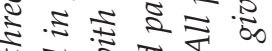

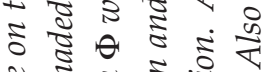

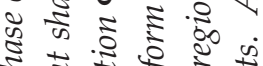

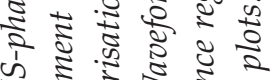

के

व

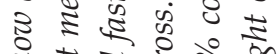

के क्षे है

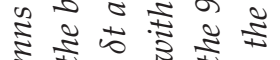
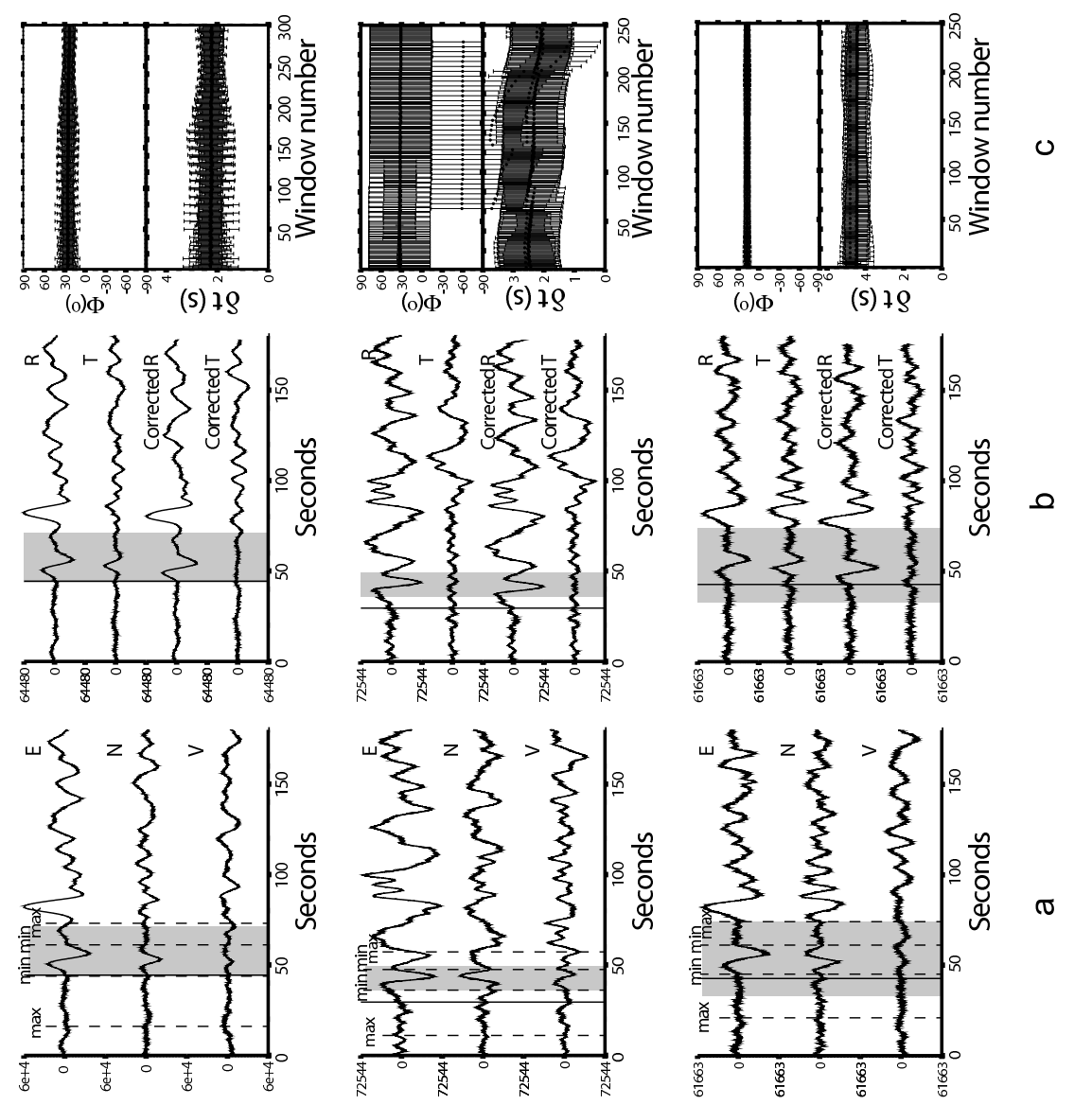

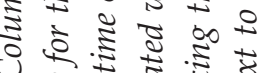

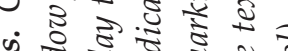

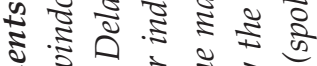

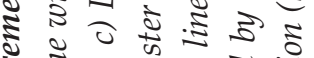

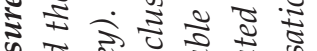

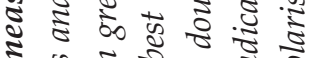

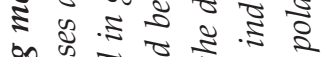

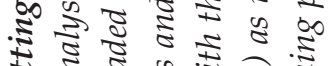

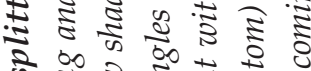

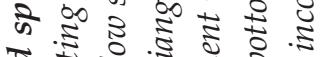

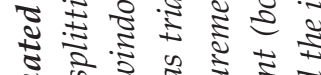

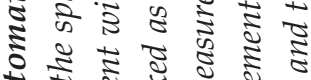

गิ ₹

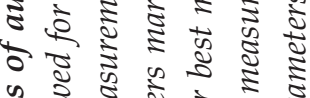

\%

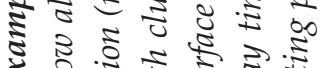

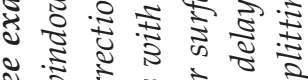

के के के के के के

है

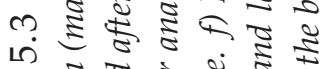

पू है है

गु.

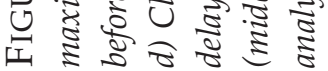


For the splitting measurements presented in this paper we used Teanby's [Teanby et al., 2004] code for automated shear-wave splitting (figure 5.3). The code is based on Silver and Chan's [Silver \& Chan, 1991] method to minimise energy on the transverse component of a split shear-wave by performing a grid search over a rotation angle and a time delay. The code performs measurements for a number of windows with different lengths and starting times within given limits around the phase arrival (figure 5.3a). The best and most stable fitting apparent splitting parameters, the polarisation azimuth of the faster shear-wave (fast polarisation) $\Phi$ and the delay time between the two shear-waves $\delta t$, are chosen by a cluster analysis (figure 5.3d). This method helps to eliminate outliers from the measurements that can be caused by including too large or too small windows around the S-arrival. We modified the code to extend its original application for local S-phases to work for splitting on teleseismic phases with known incoming polarisation.

Even though Teanby's code [Teanby et al., 2004] gives objective solutions, we additionally apply visual quality control. This is to ensure minimal energy on the transverse component after it is corrected for the preferred splitting value (figure 5.3b), stability of measurements (figure 5.3c), similarity of fast and slow waveforms (figure 5.3e), linear particle motion after correction (figure 5.3e) and a unique, well-defined error surface (figure 5.3f). Based on this quality control, results are finally rated into good (A), fair (B) and marginal (C). The example measurements in figure 5.3 were all rated good, with the top one giving "normal" splitting parameters, the middle one showing no apparent splitting and the bottom one showing the largest delay time found in this study. In the following, measurements that do not exhibit any apparent anisotropy (figure 5.3 middle) are referred to as null measurements or "nulls". Nulls are good quality measurements, which contain little energy on the raw transverse component and show error surfaces elongated in the time domain. Nulls do not necessarily mean that the medium along the travel path is isotropic. They can also occur if the incoming polarisation is parallel to the fast or the slow polarisation or if the fast axis is oriented vertically, as in these constellations the wave does not need to split. Other causes for apparent isotropy are complicated waveforms due to complex anisotropy, attenuation, scattering or noise. Complex waveforms are excluded by visual checks.

We use broadband data recorded along the NW-SE striking CNIPSE line (main deployment, 19 easternmost stations, from January to June 2001, three stations - YUPC, WULC, MILC - from July to December 2001), its western extension (WCNIPSE, seven stations deployed in November 2004: three until August 2005 and four until November 2005) and national network (GeoNet) stations (figure 5.1). The line strikes perpendicular to the Hikurangi trench and crosses the CVR. The station spacing is nominally $10 \mathrm{~km}$. The eight closest GeoNet stations surrounding the transect were considered and data was analysed from their deployment date until the end of 2005. Many of the stations have only been deployed recently (end of 2003 onwards) with stations TOZ (1998) and URZ (2001) being installed for the longest. Published SKS splitting results for these two stations [Duclos et al., 2005] were reprocessed using the automated splitting code. 


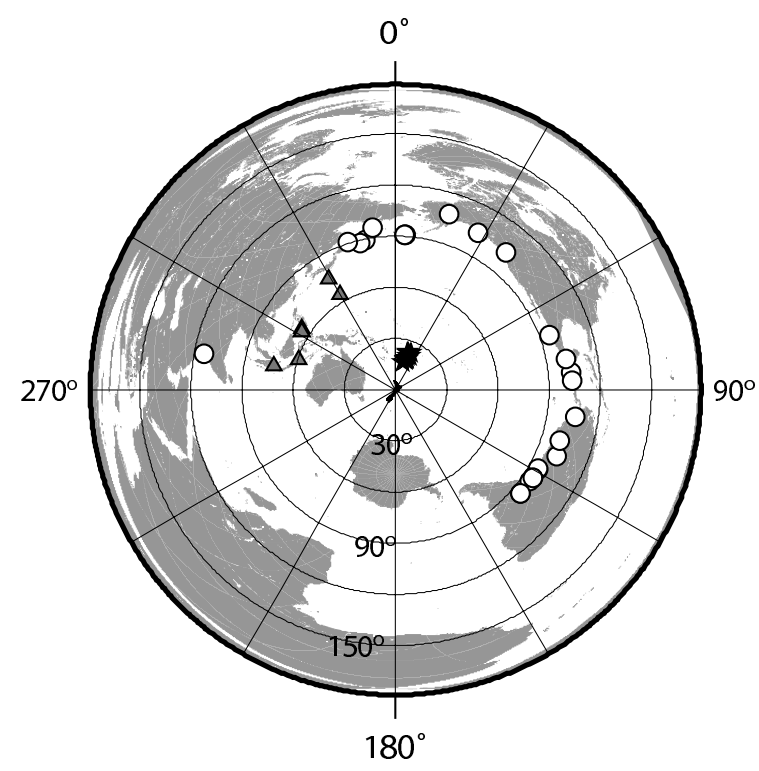

FIGURE 5.4 Splitting event map. Globe with locations of events (white circles $=S K(K) S$, grey triangles $=S$, black stars $=S c S$ ) used for shearwave splitting measurements in this study in equidistant projection centred on North Island.

All events with magnitudes greater than 5.5 and distances $\Delta>85^{\circ}$ for $\mathrm{SK}(\mathrm{K}) \mathrm{S}-,{ }^{\circ}, 60^{\circ}<\Delta<0^{\circ}$ for teleseismic S- and $\Delta<35^{\circ}$ for ScS-phases were considered for splitting measurements (figure 5.4). Backazimuthal coverage for SK(K)S-phases is $340-135^{\circ}$, whereas the coverage for S- and ScS-phases are limited to $280-330^{\circ}$ and $5-20^{\circ}$, respectively (figure 5.4). This gap in the distribution of events will prevent detailed investigation of complicated anisotropic structures such as tilted symmetry axis. In order to limit source-side anisotropy, only events deeper than $420 \mathrm{~km}(350 \mathrm{~km})$ were used for S and ScS splitting measurements, respectively. We tried to perform measurements on unfiltered data, but due to New Zealand's noisy island nature that was not always possible. Typical filters used were a Butterworth bandpass from 0.04 to $0.1 \mathrm{~Hz}$ for S- and SK(K)S-, and a $0.3 \mathrm{~Hz}$ lowpass for ScS-phases. The higher frequency filter for ScS-phases reflects the higher frequency contents of their initial waveforms, as events are closer.

\subsection{Splitting results}

We obtain a high-quality dataset of single splitting measurements from 99 SK(K)S-, 32 Sand 24 ScS-phases recorded at 38 stations with standard deviations for fast polarisations less than $12^{\circ}$ and for delay times less than $0.7 \mathrm{~s}$ (table E.1). Out of these 155 results, 86 are nulls, which do not show any apparent anisotropy.

\subsubsection{SKS- and SKKS-phases}

Along the (W)CNIPSE line and the surrounding GeoNet stations, shear-wave splitting could only be observed in middle and eastern central North Island (figure 5.2, table E.1). Fast polarisations are consistently subparallel to the trench, ranging between $7^{\circ}$ and $66^{\circ}$. The data show a slight backazimuthal dependency, as events from backazimuths between $80^{\circ}$ 
and $130^{\circ}$ give more north-south trending fast polarisations $\left(\Phi=24 \pm 2^{\circ}\right)$ than events from other backazimuths $\left(\Phi=55 \pm 9^{\circ}\right)$ (figure 5.2).

Delay times are very high, ranging from 0.85 to $4.5 \mathrm{~s}$ (figures 5.2, 5.3). They increase from the east coast towards the CVR, reaching their highest values in the central CVR (stations TETC, KIWC and POIC, figures 5.2, 5.5, 5.6). Further to the west delay times decrease towards the western border of the CVR. This behaviour is illustrated in figure 5.5 by means of a single event recorded across the CNIPSE line. For GeoNet stations the highest delay time is found at VRZ $(3.90 \pm 0.20 \mathrm{~s}$, figure 5.2). Stations URZ $(0.85 \pm 0.11 \mathrm{~s})$ and BKZ $(1.07 \pm 0.09 \mathrm{~s})$ yield the smallest (non-null) delay times.

For almost all the stations in western central North Island we obtained only null measurements. There is one exception: Reprocessed data from station WLZA yield one nonnull measurement with $\Phi=61^{\circ}$ and $\delta t=1.7 \mathrm{~s}$. Duclos [2005] found three nulls and one set of apparent splitting parameters for TOZ. After reprocessing we could verify two nulls. For Northland, Duclos et al. [2005] present mainly null measurements with only a few, mostly lower quality exceptions. Reprocessing with the automated code verified a preponderance of nulls, leaving only three sets of apparent splitting measurements with east-west fast polarisations and delay times between 0.86 and $1.86 \mathrm{~s}$. At station VRZ, we only obtained one non-null measurement with a very large delay time of $3.9 \mathrm{~s}$.

Due to different deployment times, it is not possible to compare splitting parameters for the same event at CNIPSE and WCNIPSE stations. But there are seven events recorded at GeoNet stations in Hawke Bay and Waikato, which provide a direct comparison of eastern and western stations. Two of these events (backazimuth $27^{\circ}$ and $120^{\circ}$ ) provide only nulls for all stations due to the incoming polarisation being subparallel or subnormal to the predominantly trench-parallel fast polarisation. For the other five events, positive splitting parameters were retrieved for eastern and southwestern (WAZ, VRZ) stations and nulls for the western stations (table E.1, figure 5.2).

\subsubsection{S- and ScS-phases}

For four out of six teleseismic $S$ events we obtained splitting results on more than one station (table E.1). Fast polarisations range consistently between $32^{\circ}<\Phi<56^{\circ}$ for four events, with an average of $\Phi=42 \pm 7^{\circ}$ (figure 5.2). One event at the Celebes Sea, western Pacific, gave differing fast polarisations at two stations (BKZ: $\Phi=96^{\circ}$, TSZ: $\Phi=140^{\circ}, \mathrm{WAZ}: \Phi=115^{\circ}$ ). High delay times were measured $(1.40 \mathrm{~s}<\delta t<3.45 \mathrm{~s})$ for most events. We observe the same pattern of decreasing delay times across the western border of the CVR as with SK(K)S-phases. The Hawke Bay region exhibits the highest delay times (figures 5.1, 5.2). Unfortunately, no results could be obtained for the middle section in the CVR. S-phases recorded at western central North Island stations yield only nulls.

ScS records were generally noisy. Delay times $(0.35 \mathrm{~s}<\delta t<2.25 \mathrm{~s})$ and fast polarisations 


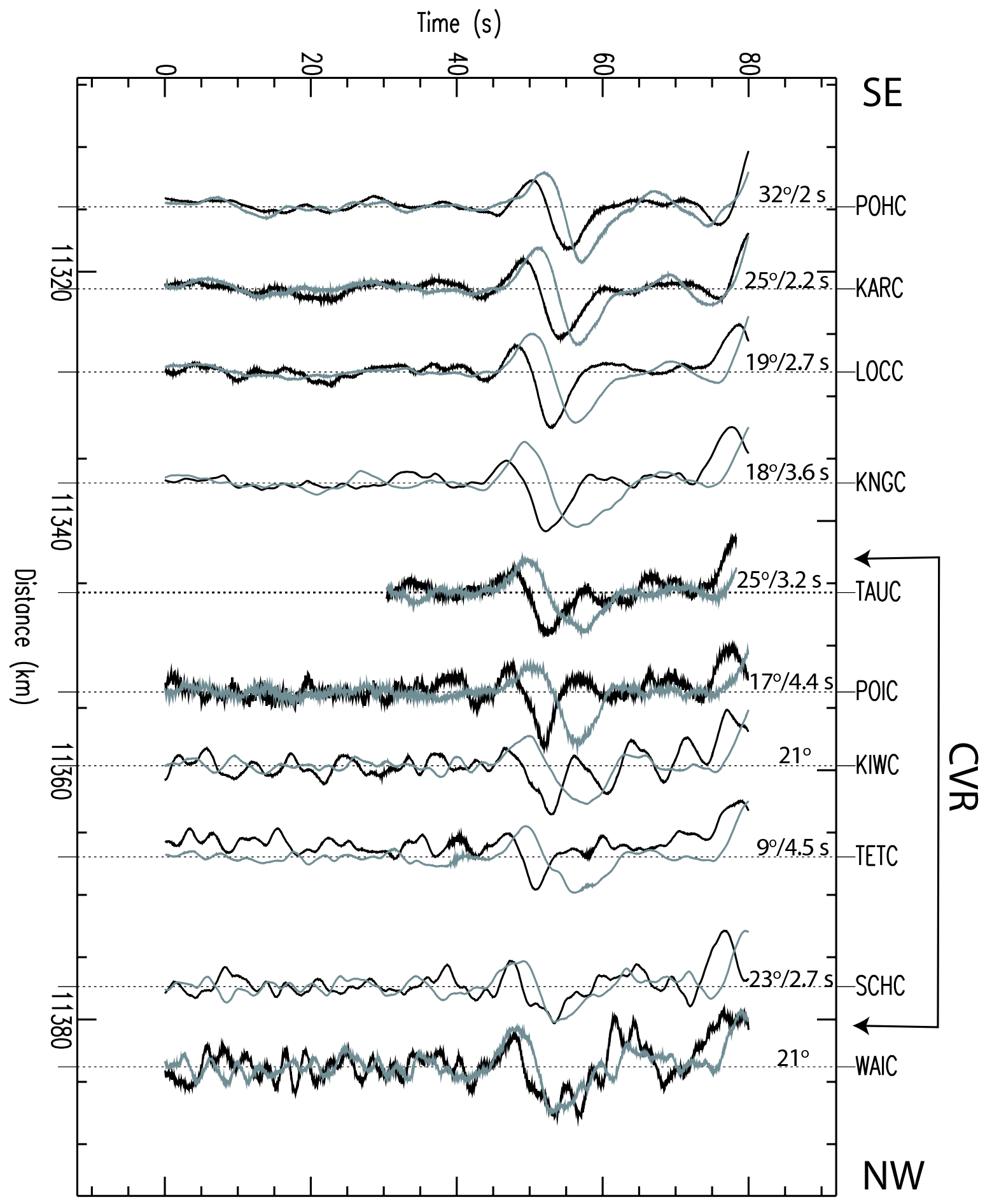

FIGURE 5.5 Record section showing unfiltered SKS-phases for event from 13.01.2001, M=7.9, BAZ=83, rotated into their individual fast (black) and slow (grey) polarisations illustrating changes in delay time across the CNIPSE line. Stations KIWC and WAIC only obtained poor quality measurements for this event and thus are rotated for a mean fast polarisation of $21^{\circ}$. Phases are lined up based on the expected arrival times from AK135. 


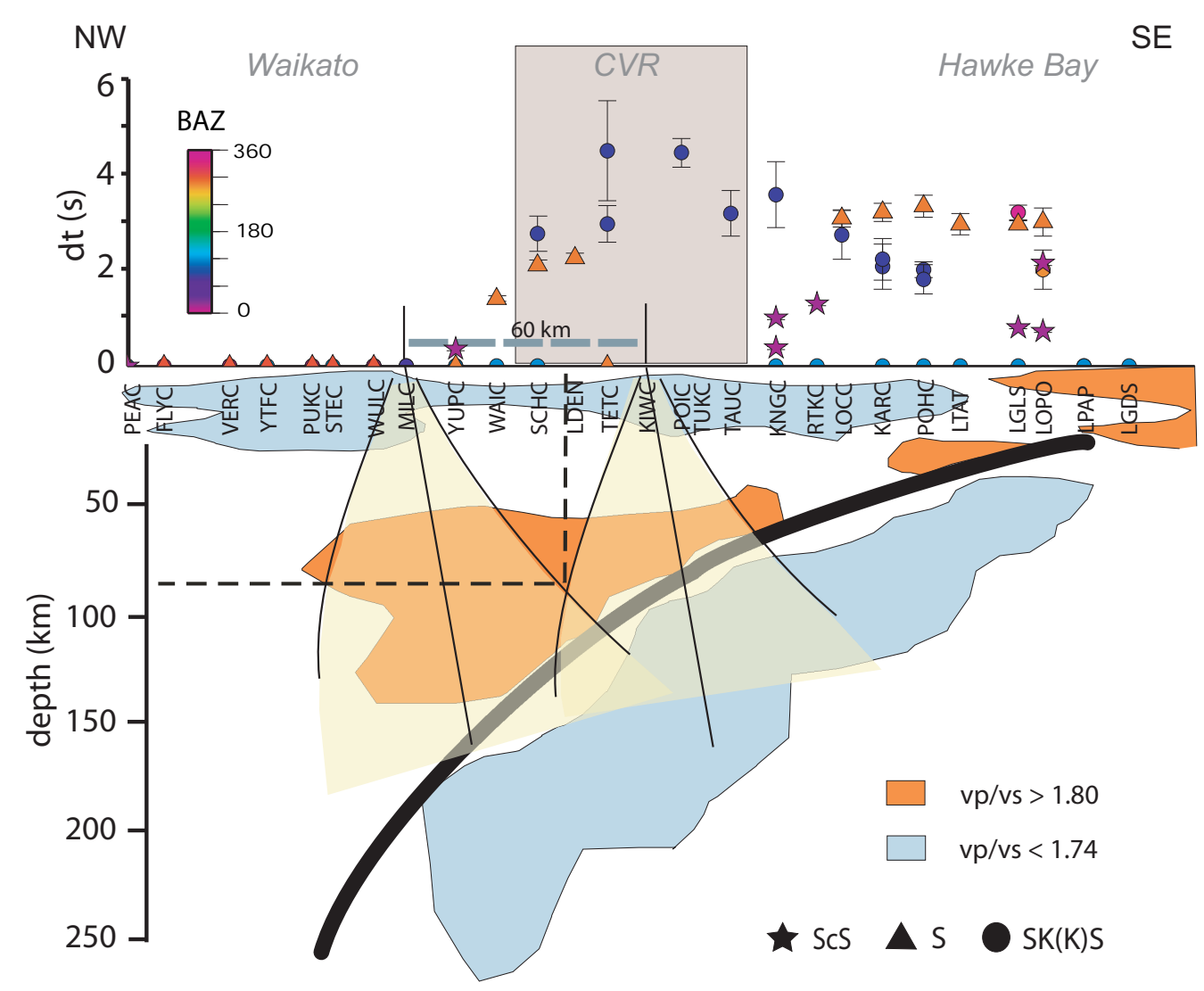

FIGURE 5.6 Cross section along (W)CNIPSE line, illustrating changes in delay time for individual events. Results are coloured according to backazimuth (BAZ). Circles represent SK(K)S, triangles S- and stars ScSphases. Tomographic cross-section after Reyners et al. [2006] showing high $(>1.80)$ and low $(<1.74) \mathrm{vp} / \mathrm{vs}$. Top of the subducted slab shown as a black solid line from Ansell \& Bannister [1996]. Yellow shaded areas represent the approximate width of Fresnel zones for the stations with largest delay times and with the closest null measurement.

$\left(3^{\circ}<\Phi<102^{\circ}\right)$ have more variability, but the average fast polarisation is consistent with other phases $\left(\Phi=44 \pm 5^{\circ}\right)$. For western central North Island, ScS-phases could only be measured at station KUZ.

\subsection{Discussion}

We use the shear-wave splitting results to determine different regions of anisotropy across the Hikurangi subduction zone (figure 5.2). The regions will be distinguished by location and mechanisms causing the anisotropy. The main observations from the splitting measurements leading our interpretation are:

- High delay times in CVR

- No apparent splitting in western central North Island

- Consistent splitting parameters for the rest of central North Island 
- Contradicting results for local S and teleseismic splitting measurements in CVR

- Backazimuthal variations in delay times

\subsubsection{Locating anisotropy}

\section{Hawke Bay}

Trench-parallel fast polarisations and average delay times around 2-3s define the forearc region in Hawke Bay (figure 5.2). Although these delay times are slightly larger, results are fairly consistent with splitting parameters from southern North Island (figure 5.2) [Gledhill \& Gubbins, 1996; Marson-Pidgeon et al., 1999]. The three exceptions, with trench-perpendicular fast polarisations (BKZ, TSZ and WAZ) are obtained from S-waves generated by the same event and can therefore be related to source side anisotropy. The consistent fast polarisations measured from SKS- and most S-phases restrict the location of anisotropy to the crust, the subducting lithosphere and the sub-slab mantle as their travel paths only overlap in these regions. The mantle wedge under Hawke Bay is thin (figures 5.1, 5.6) and its contribution to shear-wave splitting can be neglected.

Further information about anisotropy depth distribution comes from local splitting measurements. Trench-parallel fast polarisations with average delay times of $0.3 \mathrm{~s}$ define the forearc region [Audoine et al., 2004; Morley et al., 2006]. Morley et al. [2006] constrict the maximum depth of the anisotropy in eastern central North Island to $60 \mathrm{~km}$, which yields a minimum anisotropy of $1.6 \%$. We ascribe this splitting to crustal anisotropy, as delay times are small, do not vary with depth and splitting measurements tend to be more biased towards upper anisotropic layers. Additionally, we suggest that the trench-parallel anisotropy within the slab found in southern North Island [Audoine et al., 2000; Brisbourne et al., 1999; Gledhill \& Gubbins, 1996; Matcham et al., 2000] and in nearby regions [Galea, 1993] is "frozen-in" (see below) and therefore also exists under Hawke Bay. We estimate its contribution to be about $0.5 \mathrm{~s}$ as was found for the deepest earthquakes beneath Wellington [Matcham et al., 2000]. Consistent trench-parallel anisotropy in the crust and subducted lithosphere is also supported by the small delay times measured with higher frequency ScSphases, which are assumed to be more sensitive to the upper layers (figure 5.6).

As the slab and the crust can only explain a small amount of the anisotropy found with teleseismic events, most of the anisotropy has to be located in the rest of the upper mantle below the subducted slab. In order to acquire the consistently large delay times, fast polarisations in the sub-slab region must also be trench-parallel. Additional evidence for consistent trench-parallel anisotropy under Hawke Bay is provided by the null splits, which were only observed for events from backazimuths sub-parallel and perpendicular to the mean fast polarisation (figure 5.2). This suggests only one anisotropic layer, e.g. the same fast polarisations for slab and sub-slab mantle. Furthermore, we found little backazimuthal dependency of fast polarisations, inconsistent with the expected $\pi / 2$ periodicity apparent 
in two layer anisotropy models [Silver \& Savage, 1994]. A backazimuthal dependency of delay times is suggested with S-phases arriving from the west yielding higher delay times than SKS-phases from the east (figures 5.2, 5.6). With the western backazimuth and shallow incidence angle direct S-phases sample more of the region west of Hawke Bay. Thus, we relate the higher delay times of those phases arriving from the west to their longer travel path through the mantle wedge (see next section). More complicated anisotropy, e.g. with plunging symmetry axis is not needed to explain the splitting observations in Hawke Bay. Therefore, we suggest that consistent anisotropy in the crust, subducted lithosphere and the sub-slab mantle produces trench-parallel fast polarisations in Hawke Bay, in agreement with interpretations for southern North Island [Marson-Pidgeon et al., 1999].

Trench-parallel fast polarisations still predominate at stations URZ and KNZ in East Cape (figure 5.2). This suggests the anisotropic structure proposed for Hawke Bay extends further north along the subduction zone. A study in East Cape [Cochran, 1999] reported mostly trench-parallel fast polarisation with very large delay times (figure 5.2). As data were unavailable for quality assessment or reprocessing, we include it for the sake of completeness without putting too much emphasis on it.

\section{Central Volcanic Region}

With fast polarisations trench-parallel as in the Hawke Bay region, the most compelling feature of splitting parameters in the Central Volcanic Region is the very large delay times (up to $4.5 \mathrm{~s}$, figures $5.2,5.6$ ). In contrast to Hawke Bay, the mantle wedge under the CVR is considerably thicker and has to be included as a possible anisotropic region. Shear-wave splitting measurements from local shear-waves sampling the mantle wedge can help to assess its contribution to teleseismic measurements.

Strikingly, local S-waves show a $90^{\circ}$ change in fast polarisations from trench-parallel at eastern stations to trench-perpendicular in the CVR [Morley et al., 2006]. Audoine et al. [2004] present more scattered results, but with a similar trend. They find trench-perpendicular fast polarisations (extension-parallel) for deep events $(>100 \mathrm{~km})$, but trench-parallel for shallow events at short periods. Morley et al. [2006] interpret 1.6\% trench-perpendicular anisotropy down to about $100 \mathrm{~km}$ depth. This conflicts with the trench-parallel fast polarisations measured from teleseismic phases. To resolve this contradiction, instead of the suggested thick layer with small anisotropy, higher anisotropy of about $10 \%$ restricted to the thin crust or uppermost mantle can cause the local splitting results. Fresnel zone estimates support this model, as the change in fast polarisations for local earthquakes occurs over a very small distance $(\sim 10 \mathrm{~km})$. In this model a second, deeper layer in the mantle wedge is needed to explain teleseismic observations. The lack of strong backazimuthal dependency from local and teleseismic results could be due to the small contribution of the upper layer or to the orthogonal fast polarisations in the two layers. However, as discussed later, the stress regime in the extending CVR is not expected to yield trench-perpendicular crustal 
anisotropy.

Due to the time limitation of the temporary arrays, the backazimuthal coverage of our data (figure 5.4) is not sufficient to test for the patterns in delay times expected for plunging symmetry axis [Savage \& Silver, 1993]. Tilted anisotropy with plunging symmetry axis in the mantle wedge could cause constant fast polarisations but variations in delay times for different incidence angles and backazimuths. Yet, it cannot explain changing delay times across the array for a common event (figure 5.6). Moreover, the discrepancy between local and teleseismic observations remains. Alternatively, frequency dependent anisotropy could play a role (see below).

The observed strong variations in delay times along the CNIPSE line allow us to use simple Fresnel zone estimates to constrain the location of the anisotropic region under the CVR. Apparent splitting parameters depend on the period of the wave; if anisotropy varies over distances smaller than the Fresnel zone, true anisotropy can only be measured away from the boundary between different anisotropic regions [Chevrot et al., 2004]. The distance between the two stations with the largest difference in delay times for the same SKS event, TETC or POIC and MILC, is about $60 \mathrm{~km}$ (figure 5.6). Using this $60 \mathrm{~km}$ as the width of the Fresnel zone for SKS-phases (periods around 8s), restricts the source of the anisotropy to be shallower than $80 \mathrm{~km}$ [Alsina \& Snieder, 1995], as Fresnel zones for the two stations overlap at greater depths. This is on the order of the slab depth under the CVR and therefore in the mantle wedge. There are insufficient data from ScS-phases, which might otherwise provide better resolution of the lateral boundary due to their much shorter periods. S-phases used for splitting measurements only have slightly shorter periods than $\mathrm{SK}(\mathrm{K}) \mathrm{S}$ and $\mathrm{S}$ measurements are lacking in the CVR, thus we cannot use them for further Fresnel zone estimates. However, as delay times measured for S-waves with a longer travel path through the mantle wedge (see previous section) are higher than those for SKS-phases to the same stations (figure 5.6), they provide further evidence for a highly anisotropic region in the mantle wedge. This region could have sharp boundaries that appear as smooth delay time variations on the surface because of the averaging effects of the Fresnel zones [Chevrot et al., 2004; Fischer et al., 2005].

\section{Waikato}

In contrast to the rest of central North Island, Waikato is exclusively characterised by null measurements (figures 5.1,5.2). They make the region appear isotropic and imply an abrupt change in the anisotropic structure compared to the adjacent CVR. Decreasing delay times across the western border of the CVR (figure 5.6) support the interpretation of a laterally bounded block of high anisotropy under the CVR and little or no apparent anisotropy under Waikato. Due to the width of the Fresnel zone and non-vertical incidence angles, splitting measurements are still obtained west of the block, but delay times decrease steadily, faster for events with westerly backazimuths (figure 5.6). The southern boundary of the appar- 
ently isotropic zone extends to approximately $39^{\circ} \mathrm{S}$, where station VRZ measured mainly nulls with one single measurement with high delay time (figure 5.2). The coexistence of this large measurement with nulls could be related to lateral variations and indicate that the station is located above an anisotropic boundary [Fischer et al., 2005]. An earlier study [MarsonPidgeon et al., 1999] found trench-parallel fast polarisations at a station only $20 \mathrm{~km}$ southwest of VRZ (figure 5.2), but two of the measurements were of marginal quality. Therefore, we define the mantle below Waikato, bounded by the CVR to the east and by about latitude $39^{\circ} \mathrm{S}$ to the south, as apparently isotropic. The southern boundary of the region nearly coincides with a main structural boundary, which connects Mt. Taranaki with the southern tip of the CVR and is interpreted as the transition from extensional to compressional backarc regimes [Stern et al., 2006].

North of Waikato, only two non-null fast polarisations with small delay times were measured, suggesting either the continuation of the apparent isotropy or a change to lower eastwest oriented anisotropy for Northland [Duclos et al., 2005].

\subsubsection{Causes for anisotropy}

\section{Olivine type-A fabric}

In olivine type-A fabric, the lattice-preferred orientation (LPO) of the fast olivine a-axis aligns with the extension direction, which leads to shear-parallel a-axes in the infinite strain limit, or axes parallel to asthenospheric flow [e.g. Savage, 1999]. It is the most frequently observed and accepted olivine fabric and has been used for many interpretations of splitting observations, especially under stable continental areas [Ismaiil \& Mainprice, 1998; Ribe, 1989]. Type-A olivine reaches an anisotropic strength of 5.5\% [Jung \& Karato, 2001a] for S-waves. However, addition of other minerals and the likelihood of imperfect alignment reduce anisotropy quickly. On the other hand, fabric strength in the Earth is suggested to be stronger than measured experimentally because of the finer grain sizes due to larger stress in experiments (B. Holtzman, pers. communication). An average value of $4 \%$ mantle anisotropy is widely accepted [Savage, 1999].

Asthenospheric flow is considered as one of the possible causes of LPO in the mantle [Savage, 1999]. The similarity of shear-wave splitting measurements along the eastern side of North Island (figure 5.2) prompts the idea of a common source. Trench-parallel mantle flow beneath the slab with contributions from the subducting lithosphere has been suggested to explain anisotropy measured in southern North Island [Gledhill \& Gubbins, 1996]. The continuation of this pattern under eastern central North Island is sufficient to explain fast polarisations observed in Hawke Bay and can account for the observed delay times of maximum $3 \mathrm{~s}$ in Hawke Bay with about $300 \mathrm{~km}$ of anisotropic material. Trench-parallel mantle flow can arise from oblique subduction relative to the plate motion (figure 5.1), from the steepening of the slab towards the south [Anderson \& Webb, 1994] or from retrograde motion of 
the slab indicated by the extension of the CVR. Below temperatures of about $900^{\circ} \mathrm{C}$ olivine alignment can no longer occur due to current deformation [Estey \& Douglas, 1986; Silver \& Chan, 1988]. Thus, the contribution of the colder subducting slab is probably due to fossil anisotropy with "frozen-in" LPO caused by earlier deformation (cf. section 5.5.1).

In the case of ductile stretching of the lithosphere in the mantle wedge, fast polarisations in the CVR should align with extension. Although this is sufficient to explain fast polarisations from local studies [Audoine et al., 2004; Morley et al., 2006], type-A olivine due to extension cannot explain trench-parallel (extension-perpendicular) fast polarisations observed with teleseismic events in the CVR (figure 5.2).

To explain the $4.5 \mathrm{~s}$ splitting in the CVR, we invoke the same $3 \mathrm{~s}$ in and under the slab as for Hawke Bay plus an additional $1.5 \mathrm{~s}$ in the mantle wedge. In an $80 \mathrm{~km}$ thick mantle wedge this would require $7.5 \%$ anisotropy. Even though trench-parallel flow in the mantle wedge has been suggested [Audoine et al., 2004; Marson-Pidgeon et al., 1999] for depths beneath 100-150 km, this does not agree with the established corner flow model [Buttles \& Olson, 1998; Funiciello et al., 2006; Kincaid \& Griffiths, 2003]. We therefore conclude that type-A olivine in the subducted slab and the mantle below can account for most of the observed anisotropy in Hawke Bay and the CVR. However, this fabric is not sufficient to cause the high anisotropy needed in the mantle wedge under the CVR.

\section{Other olivine fabrics}

Under the influence of water and varying pressure and temperature conditions, olivine develops LPO different from type-A. Type-B fabric is found under high $\mathrm{H}_{2} \mathrm{O}$ content and moderate stress conditions (>300 MPa) [Jung et al., 2006] and is of particular interest, as the olivine a-axis orients perpendicular to maximum principal strain. Type-B olivine in the mantle wedge has been invoked for interpretation of splitting measurements at subduction zones worldwide [Long \& van der Hilst, 2006; Nakajima \& Hasegawa, 2004; Schmid et al., 2004; Volti et al., 2006] and for the Hikurangi subduction zone [Audoine et al., 2004; Duclos et al., 2005]. With an average of $7.6 \%$, its S-wave anisotropy is higher than type-A (5.5\%). Even higher anisotropy (12\%) was found for type-E olivine [Katayama et al., 2004]. This fabric occurs under the influence of $\mathrm{H}_{2} \mathrm{O}$ and low stresses. Like type-A fabric, LPO orients subparallel to stress. However, the experimentally determined fabric strengths cannot unconditionally be extrapolated to the Earth, as mentioned in the previous section (5.5.2), and the exact causes for the development of the different type fabrics are still unknown.

The occurrences of type-B olivine with trench-perpendicular corner flow under the CVR would lead to trench-parallel fast polarisations as observed for SKS (figure 5.2). Type-B olivine over $80 \mathrm{~km}$ mantle wedge can account for the observed delay times. However, the shear stress in the mantle wedge is unlikely to meet the required condition. A simple calculation using the lithostatic pressure at $100 \mathrm{~km}$ depth acting on the top of the slab gives an upper limit of $200 \mathrm{MPa}$. Estimates from numerical modelling show even lower shear stresses in the 
mantle wedge [e.g. Lamb, 2006]. Lower stresses and high delay times in the CVR support the idea of type-E fabric, but again only in the presence of trench-parallel mantle flow, which we consider unlikely, as discussed earlier. Consequently, these other olivine fabrics cannot provide a sufficient explanation for the anisotropy needed in the mantle wedge.

\section{Aligned cracks}

The alignment of cracks or fractures is another effective mechanism to create seismic anisotropy, if their spacing is much smaller than the seismic wavelength. The strength of the anisotropy depends on the shape of the inclusion and the velocity contrast of inclusion and surrounding matrix. The fast polarisation is parallel to the alignment of the cracks, which will be the orientation of the maximum horizontal stress. However, vertical stresses cause cracks to close and confine fluid- or air-filled cracks to the brittle crust.

Crustal thickness under the CVR reaches only about $20 \mathrm{~km}$ [Stratford \& Stern, 2004]. With an estimate of $6 \%$ anisotropy the crust can accommodate $0.3 \mathrm{~s}$. This is within the order of the uncertainties of our measurements. Cracks in the crust would orient perpendicular to extension, i.e. trench-parallel in the CVR. Therefore, local shear-wave splitting should yield trench-parallel fast polarisations, which does not fit observations by Morley et al. [2006]. Consequently, crustal anisotropy due to aligned cracks cannot cause the observed splitting results in the CVR.

In the Hawke Bay region the crust is thicker than in the CVR and the region is not governed by the extensional regime of the CVR. Local shear-wave splitting measurements suggest a positive contribution of about $0.2 \mathrm{~s}$ crustal anisotropy with trench-parallel fast polarisation caused by the flexure of the overriding Australian plate [Morley et al., 2006].

\section{Melt}

Similar to the alignment of cracks, the shape-preferred orientation (SPO) of disk-shaped inclusions (lenses or dikes) generates seismic anisotropy. Due to the large velocity contrast, this mechanism is especially effective, if inclusions are filled with melt. Magma can be present throughout the entire upper mantle, holding the possibility of producing large delay times. Modelling anisotropy due to melt-filled cracks for regions beneath mid-ocean ridges, Kendall [1994] found shear-wave delay times over 2.5 s. Ayele et al. [2004] show delay times dependent on percentage melt fraction, crack aspect ratio and layer thickness. For example, a $90 \mathrm{~km}$ thick layer of $2 \%$ melt in inclusions with an aspect ratio of 0.05 can produce more than $2 \mathrm{~s}$ delay time. Analogous to oriented cracks, we expect fast polarisations due to magma-filled lenses to be trench-parallel. Even though this seems to be a plausible explanation for our splitting observations in the CVR, the contradiction with local splitting results [Morley et al., 2006] remains. 


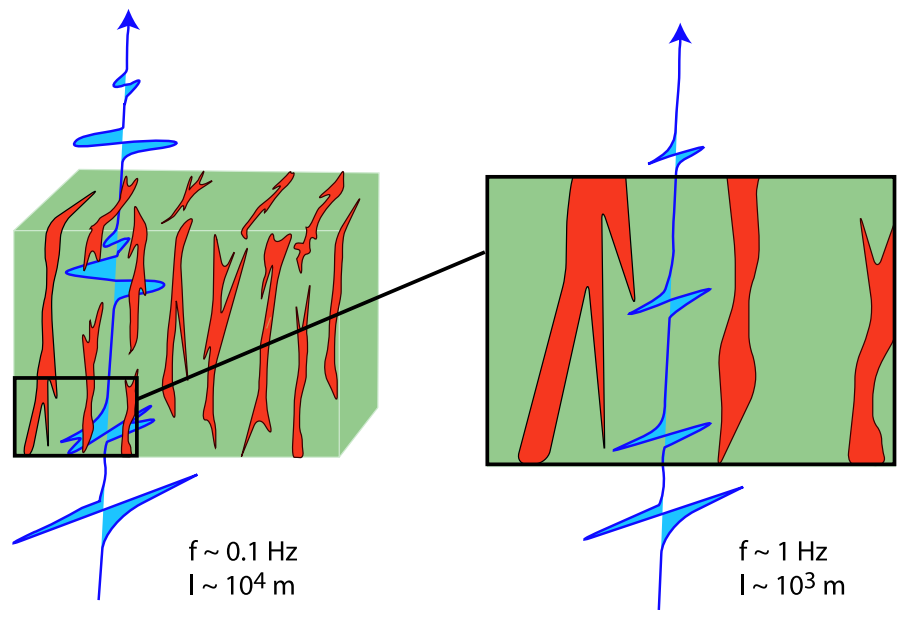

FIGURE 5.7 Frequency dependent shear-wave splitting. Illustration of the proposed source for frequency dependent anisotropy. Low frequency teleseismic waves split while propagating through material with aligned bands, if the band spacing is smaller than the wavelength $l$ (left). Defocusing of the lower velocity melt bands causes higher frequency local phases, with shorter wavelength to image the depleted matrix with different anisotropic characteristics (inset, right). $f:$ frequency.

Holtzman et al. [2003a] suggest another mechanism for the influence of melt on anisotropy. They observed a switch in a- and c-axes, similar to Jung \& Karato [2001b]. But the mechanism for the switch is not a change in the slip system, but strain partitioning in the partially molten medium. The coexistence of both mechanisms is unlikely due to partitioning of water into the melt [Holtzman et al., 2003a]. In their study, Holtzman et al. [2003a] consider the presence of up to $6 \%$ melt. Such melt fractions can produce high anisotropy in regions of active volcanism or rifting [Kendall, 2000]. Rift-parallel fast polarisations and high anisotropy due to the segregation of melt was interpreted at the Ethiopian rift [Kendall et al., 2005]. The unusually high delay times in the extending CVR were measured at stations located in the presently active TVZ. If melt aligned in bands under the CVR can generate high anisotropy (e.g. $7.5 \%$ over $80 \mathrm{~km}$ ), then it can explain the increase in splitting delay times of maximum $1.5 \mathrm{~s}$ observed in the CVR.

The presence of melts in the mantle wedge under the CVR is apparent from the active volcanism in the area and can be ascribed to a lowered solidus caused by the dehydration of the subducting slab [e.g. Stern et al., 2006]. Evidence for melt under the CVR comes from several geophysical studies. Active seismic data show a strong reflector at $35 \mathrm{~km}$ depth, which has been interpreted as a melt pool of 1.2\% partial melt [Stratford \& Stern, 2006]. Pn speeds are significantly reduced (to less than $7.4 \mathrm{~km} / \mathrm{s}$ ) beneath the CVR [Haines, 1979; Seward et al., 2006; Stratford \& Stern, 2006] and $v p / v s$ ratios (figure 5.6) are high [Bannister et al., 2004; Reyners et al., 2006]. Both observations have been explained in terms of the presence of partial melts. Further indications are given by radial uplift [Pulford \& Stern, 2004] and attenuation studies [Salmon et al., 2005].

Seismic anisotropy due to melt segregation allows the possibility for both trench-parallel and trench-perpendicular fast polarisations to coexist in the mantle wedge. This can cause the frequency dependence of the measurements (figure 5.7). For splitting to occur due to melt segregation, the spacing between bands of partial melt has to be smaller than the wavelength of the shear-wave (on order of $\mathrm{km}$ ) and the area affected by melt segregation has to be larger than the wavelength. Suggested band spacings of up to $500 \mathrm{~m}$ fit in with field observa- 
tions [Holtzman et al., 2003b]. If the spacing between bands of partial melt were on the order of kilometres, they would influence teleseismic phases with wavelengths of about $10^{4} \mathrm{~m}$ (frequencies $f \sim 0.1 \mathrm{~Hz}$ ), but not local phases with maximum wavelengths of $10^{3} \mathrm{~m}$ (figure 5.7). The low velocity regions defocus seismic energy, so that local phases with frequencies of $1-10 \mathrm{~Hz}$ would then only image the depleted material in the matrix, which might have extension parallel anisotropy. Further evidence for frequency dependent behaviour appears in the record section (figure 5.5). The waveforms indicate lower frequency content for the slow waves than for the faster ones, suggesting possible attenuation anisotropy.

Our preferred model to explain the anisotropy observed across the subduction zone in central North Island is illustrated in figure 5.8. We ascribe the consistent trench-parallel anisotropy in Hawke Bay and eastern central North Island to type-A olivine with about $4 \%$ anisotropy due to trench-parallel mantle flow extending over a depth of $300 \mathrm{~km}$. Contributions of crustal anisotropy due to aligned cracks and slab anisotropy, both with trenchparallel fast polarisations are likely. Under the CVR, we suggest high, frequency dependent anisotropy in the mantle wedge due to aligned melt bands.

\subsubsection{Causes for apparent isotropy}

The null observations in western central North Island illustrate a dramatic change to the consistent pattern of large splitting delay times acquired from teleseismic anisotropy studies to date in most of New Zealand. Observation of null splitting from different incoming polarisations in Waikato makes the region apparently isotropic. We emphasise here that delay times smaller than $0.5 \mathrm{~s}$ are not resolvable with the method applied and will therefore be considered as nulls.

Apparent isotropy is generally attributed to isotropic conditions, multiple layers or complex anisotropy, vertical orientation of anisotropy, or an incoming wave polarisation along either the fast or the slow axis. Events with different backazimuths and incoming polarisations showed the same null behaviour. Therefore, this rules out the explanation of null measurements being caused by incoming polarisations parallel to fast or slow axes.

Null birefringence has been observed in several studies before. If the region of apparent isotropy is narrow, it is often interpreted as the transition zone between lateral variations of differently oriented horizontal anisotropy [Anglin \& Fouch, 2005; Plomerová et al., 2001]. In Northern Apennines, null splitting occurs above a boundary between fast and slow tomographic anomalies [Plomerová et al., 2006]. Results from waveform modelling confirm the occurrence of nulls above lateral boundaries [Fischer et al., 2005]. However, the region of null measurements in the Waikato is too wide to act as a boundary zone itself. In addition, we could not identify a region of differently oriented anisotropy adjacent to Waikato in the west.

Özalaybey \& Chen [1999] attribute null birefringence in Australia to azimuthal isotropy 
with a vertical slow axis for the upper mantle of the Indo-Pacific plate, related to its unique tectonic history and fast absolute plate motion. Our null observations in Waikato, those from southern Himalaya mentioned earlier and additional ones from Hyderabad [Chen \& Özalaybey, 1998] seem to support the idea of azimuthal isotropy of the Indo-Pacific upper mantle. Conflicting results come from a recent study of shear-wave splitting that found varying fast polarisations in Australia [Heintz \& Kennett, 2005]. However, the authors identify the thick lithosphere as the main source of the observed anisotropy.

Babuška et al. [2002] identify a broader region of null measurements under the southeastern French Massif Central and attribute it to extension followed by lithospheric thinning. The weakened, probably partly detached lithosphere would then result in destruction of the original mantle fabric giving little anisotropy. Similar conclusions were drawn from Sandvol et al. [1997], who observed a lack of shear-wave splitting in southern Himalayas and Tibet. They associate the thickening of the lower Indian lithosphere with a gravitational instability. The downwelling lithosphere causes convection and steep plunging shear strain, leading to subvertical fast axes.

Morley et al. [2006] attribute anisotropy under western central North Island to the absolute plate motion, but delay times are small and can be accommodated in the crust. Reyners et al. [2006] propose a well-developed return flow under the TVZ that changes under western central North Island due to variations in the crustal thickness. Vertical return flow under western central North Island can develop hexagonally symmetric anisotropy with a vertical a (fast) axis [Savage, 1999]. Furthermore, the subducted slab plunges steeply under Waikato before it ends roughly below the west coast, suggesting an end of the mantle wedge dynamics. These changes in mantle and return flow could also generate small-scale convection [Reyners et al., 2006]. If any anisotropy forms under these conditions, we expect it to be complex and therefore not resolvable by splitting measurements, i.e. resulting in apparent isotropy.

In central North Island, the outline of the apparently isotropic region coincides with a major crustal and mantle boundary [Stern et al., 2006]. Crustal thickness increases by about $8 \mathrm{~km}$ south of Waikato [Salmon et al., 2006] and decreases towards the CVR, east of Waikato [Stern et al., 1987]. Changes across this boundary area are also apparent in seismic attenuation with mantle Q-values around 700 in Waikato dropping to 250 in the CVR and increasing south to greater than 1000 [Salmon et al., 2005]. Probably the most obvious feature distinguishing Waikato from the rest of North Island is its positive isostatic gravity anomaly [Stern et al., 1987]. Based on these and other anomalies, e.g. low upper mantle velocities, Stern et al. [2006] recently suggested convective removal of mantle lithosphere under western North Island in the Pliocene. Even though the thickened mantle lithosphere has been detached, an ongoing large-scale mantle disturbance is to be expected. Vertical and return flow patterns or small-scale convection are possible, but modelling is needed to specify mantle flow patterns in delaminated mantle. 


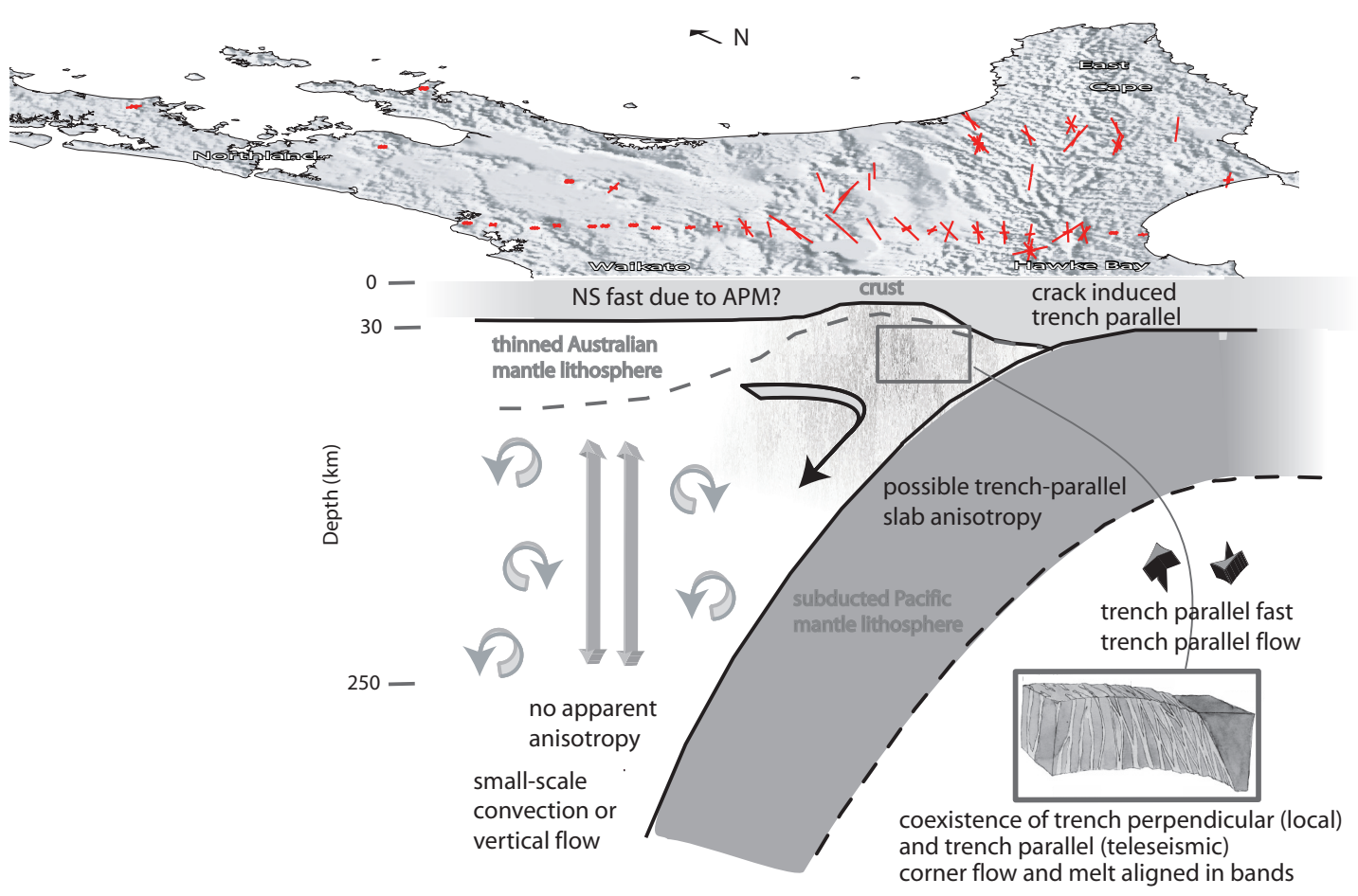

FIGURE 5.8 Model for anisotropic regions across North Island. Model showing proposed anisotropic regions across the Hikurangi subduction zone. Big arrows below the slab under Hawke Bay indicate trenchparallel mantle flow (in or out of the page) causing trench-parallel fast polarisation. Shaded area in the mantle wedge represents partial melt, possibly segregated in bands (see insert, from Holtzman) with the arrow indicating corner flow. Arrows under Waikato illustrate possibilities of small-scale convection or vertical flow. Crust-mantle boundary sketched from Stratford \& Stern [2006], top of subducted Pacific plate from Ansell $\mathcal{E}$ Bannister [1996].

\subsection{Conclusions}

Shear-wave splitting measurements across the Hikurangi subduction zone reveal strong anisotropy with rapid lateral changes. Similarity of results divides North Island into three different regions across the subduction zone (figures 5.2, 5.6). In the forearc region, eastern central North Island, consistent trench-parallel fast polarisations with average delay times of $2.5 \mathrm{~s}$ suggest type-A LPO with trench-parallel sub-slab flow (figure 5.8). Minor contributions $(0.3 \mathrm{~s})$ appear from crustal anisotropy caused by trench-parallel cracks in the overriding plate. Trench-parallel anisotropy in the slab can also account for parts $(0.5 \mathrm{~s})$ of the splitting.

Towards the backarc region, in the CVR, delay times increase to a maximum of $4.5 \mathrm{~s}$ with fast polarisations remaining trench-parallel. Fresnel zone estimates establish the cause of this increase to be in the mantle wedge. This observation conflicts with local splitting measurements, which found trench-perpendicular fast polarisations in the CVR. Two horizontal layers of perpendicular anisotropy under the CVR are possible but unlikely, as this would 
require very high anisotropy in the lower layer and a non-crustal upper layer. We therefore prefer a model of frequency dependent anisotropy due to aligned melt bands in the mantle wedge with strong anisotropy under the centre of the extending CVR (figure 5.8). An estimate for the anisotropy is $7.5 \%$ through $80 \mathrm{~km}$ in the mantle wedge. The different frequency content of fast and slow waves also suggests more complicated processes may cause the high delay times in the CVR. A more detailed study of the observed anisotropic attenuation is necessary to better understand those processes.

Western central North Island differs from the rest of North Island by revealing no apparent anisotropy. We propose that the influence of mantle wedge dynamics ends under the western border of the CVR. Different mantle flow dynamics take place under western central North Island in terms of small-scale convection or vertical mantle flow. The change in mantle flow can be ascribed to changes in lithospheric thickness, possibly caused by convective removal of thickened lithosphere. 


\section{Chapter 6}

\section{Modelling large-scale seismic anisotropy across central North Island}

As discussed in chapter 5, seismic anisotropy across the Hikurangi subduction zone exhibits strong lateral changes over distances of less than $100 \mathrm{~km}$. Teleseismic S-phases show trench-parallel fast orientations across the forearc and backarc region, with increasing delay times (section 5.4). In the CVR, delay times reach up to $4.5 \mathrm{~s}$, one of the largest delay times measured in the world. Delay times decrease systematically in the backarc region. In the far backarc, no apparent anisotropy for teleseismic S-phases is observed. In contrast, local S-phases exhibit a distinct change from trench-parallel fast polarisations in the forearc to trench-perpendicular in the backarc, with average delay times of $0.35 \mathrm{~s}$ [Morley et al., 2006; Audoine et al., 2004].

The observed pattern of anisotropy was ascribed to three different anisotropic regions across the subduction zone:

1. In the forearc region, trench-parallel fast orientations and delay times of $2-3 \mathrm{~s}$ are attributed to trench-parallel mantle flow below the slab with possible contributions from anisotropy in the slab.

2. High, frequency dependent anisotropy in the mantle wedge together with the sub-slab anisotropy add up to cause the high delay times observed in the CVR.

3. In the far backarc region, contribution to anisotropy from the mantle wedge dynamic seems to end. The apparent isotropy implies different dynamics. Possibilities include vertical mantle flow or small-scale convection.

This chapter uses different approaches to model the observed anisotropy across the Central Volcanic Region (CVR) and to test the proposed hypothesis of the different anisotropic regions. Analytical modelling approaches try to fit data with simple two-layered anisotropy 

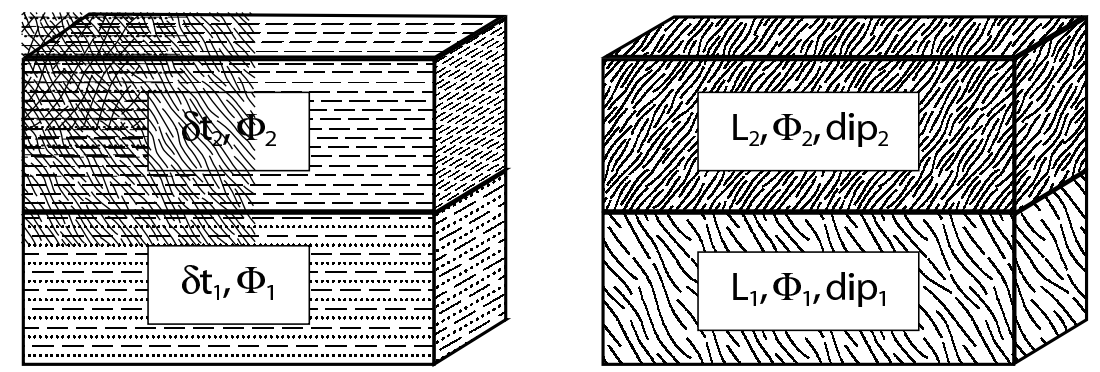

FIGURE 6.1 Two approaches for two-layer modelling: Assuming a fixed horizontal symmetry axis (left) and adding a third model parameter for dipping symmetry axis (right).

models with horizontal or dipping symmetry axes. Numerical finite difference models supply anisotropic wave propagation in 2D and 3D. These models allow setting up domains with different anisotropic characteristics such as fast orientation direction and anisotropic strength.

\subsection{Two-layer modelling}

Models of two horizontal anisotropic layers with different fast polarisation and delay time were among the first ones used to explain variations in shear-wave splitting observations with incoming polarisation [Silver \& Savage, 1994; Rümpker \& Silver, 1998; Levin et al., 1999; Hartog \& Schwartz, 2000]. Those models are based on the idea that either crust and upper mantle (for local splitting measurements) or fossil anisotropy in the lithosphere and flow induced anisotropy in the asthenosphere (for teleseismic measurements) have different characteristics.

In a first attempt to model shear-wave splitting observations from central North Island, two different kinds of two-layer models were applied; the first one includes only layers with horizontal symmetry axis and the second one allows for dipping symmetry axis. The shear-wave splitting measurements from section 5.4 are modelled as the full data set and in sub-data sets according to the location of the recording station and/or the phase used for the measurement (figure G.1). Three different best-fit criteria were applied, accounting for fit in fast polarisations, delay times and the sum of both. Despite the alternative modelling approaches, no convincing two-layer models for central North Island could be found. Thus, only the main results are summarised here. A detailed description of the modelling is given in appendix G.

The most general two-layer modelling with horizontal symmetry axis yields similar fast polarisations for the best two-layer model for the complete data set. Supported by the narrow confidence regions, these two layers are considered as basically one single layer of about 30-40 fast orientation and 1.2-2 s delay time (figure G.2). This modelling result stays the same for modelling the southeastern North Island after splitting up the data set into different anisotropic regions (figure G.4). For the CVR, best model results vary strongly with 
the best-fit criteria applied and no consistent model is found. This indicates a more complex structure under the CVR, not describable with two layers of horizontal symmetry. It also rules out the discussed possibility of two layers causing the differences in fast polarisations observed between local and teleseismic phases ( $c f$. section 5.6). However, the fast polarisation of $30^{\circ}$ determined for the lower layer from the best total fit model is consistent with the general model fitting the full data set. For western North Island, all best-fit models prefer perpendicular layers with similar delay times. This can be interpreted as apparent isotropy, since delay times for perpendicular layers subtract and therefore cancel out. This is consistent with the interpretation of the shear-wave splitting measurements given in chapter 5 .

In the second two-layer modelling approach allowing for dipping symmetry axes gives one more degree of freedom in the models and three times as many parameters to solve for. The best-fit solutions for all models do not fit the data well and are poorly constrained (figure G.5). However, for the full data set, the best-fit model consists of one dominant non-dipping layer (travel path length of $280 \mathrm{~km}$ ), with a fast orientation of $40^{\circ}$ and a negligible second layer with a path length of $40 \mathrm{~km}$. This verifies the preference for a single, non-dipping layer to describe the full data set as already found with the model approach using horizontal symmetry axis. Also in agreement with the model with horizontal symmetry axis, the preferred solution allowing for dipping symmetry for western North Island consists of two perpendicular layers with similar travel path lengths, equivalent to apparent isotropy. Results for the CVR suggest steeply dipping axes, but cannot fit the high delay times observed. Overall, the introduction of dipping symmetry axis provides no improvement in fitting observations.

Results from the two-layer modelling suggest that two layer models cannot conclusively describe shear-wave splitting observations across central North Island. New modelling approaches have to be applied to improve the fit between model and data. They should be able to account for the complex geometry of the subduction zone setting across central North Island.

\subsection{Finite difference modelling}

Seismic numerical modelling provides the opportunity to simulate wave propagation in the Earth, in order to predict seismograms expected for any given structure. The finite difference (FD) method solves the anisotropic elastic wave equation in three dimensions and provides full waveform solutions. Those synthetic waveforms can then be used in the same processing procedures as real data and compared to results from recorded data. This section gives a basic introduction into the method with special references to the code applied [Boyd, 2006], followed by its application to modelling anisotropic wave propagation in central North Island. 
Anisotropic modelling has long been confined to analytical modelling, as the solution of the anisotropic wave equation requires large computational power. Analytical models only allow for simple geometries such as flat layers (cf. section 6.1), and are therefore not suitable for modelling complex tectonic settings, such as subduction zones, where flat layers are hardly expected. The advantage of finite difference methods is that they allow one to implement any arbitrary shape of structure. They also produce full waveforms, including reflected, converted and diffracted waves. Those waveforms can be processed in exactly the same way as the real data and therefore provide the possibility of direct comparison. A main advantage of the finite difference method is the full wave propagation through the model space. Propagation can cause modifications to the waveform that are not considered in analytical models. They are, however, crucial to anisotropy measurements based on shear-wave splitting using waveform correlation. Using waveform modelling with a multi-domain, pseudospectral method, Fischer et al. [2005] find that shallow anisotropic layers generally increase measured delay times and that delay times accumulated from greater depth never get eliminated. This contradicts the theory of cancelling delay times by perpendicular layers. Full waveform modelling also considers the effect of Fresnel zones [Alsina \& Snieder, 1995], leading to larger sampling areas for lower frequency waves. Thus, lateral variations can lead to unexpected shear-wave splitting results due to "smearing" effects on the surface. On the other hand, the effects of anisotropy on the wave can get lost after propagation through isotropic media, an effect called "wavefront healing" [Fischer et al., 2005].

This shows that, in general, splitting parameters cannot be estimated solely based on the knowledge of the existing anisotropic structure. Actual shear-wave splitting measurements on synthetic waveforms allow more realistic comparison to real splitting parameters, particularly for teleseismic observations, where Fresnel zones are large and sampling occurs over long travel path through the mantle. Modelling exclusively the expected anisotropic structure of a region can be sufficient to predict local shear-wave splitting parameters [e.g. Buttles \& Olson, 1998; Lassak et al., 2006; Kneller et al., 2005, 2007], but to compare models to teleseismic observations, full wave propagation followed by shear-wave splitting measurements is essential. The main disadvantages of the finite difference method are numerical instabilities, the influence of the boundary conditions and the computer resources required (cf. section 6.2.1). This impedes the inclusion of local phases and small-scale structures into large-scale finite difference models

\subsubsection{Theory}

The seismic wave equation is derived from the basic equation of motion or mass balance $(F=m a)$ and can be expressed in terms of the density of the mass $\rho$, the displacement $u$ and the stress $\sigma$ as:

$$
\frac{\partial \sigma_{i j}}{\partial x_{j}}+f_{i}=\rho \frac{\partial^{2} u_{i}}{\partial t^{2}}
$$

It shows that stress and displacement are related via partial derivatives in time and space. 


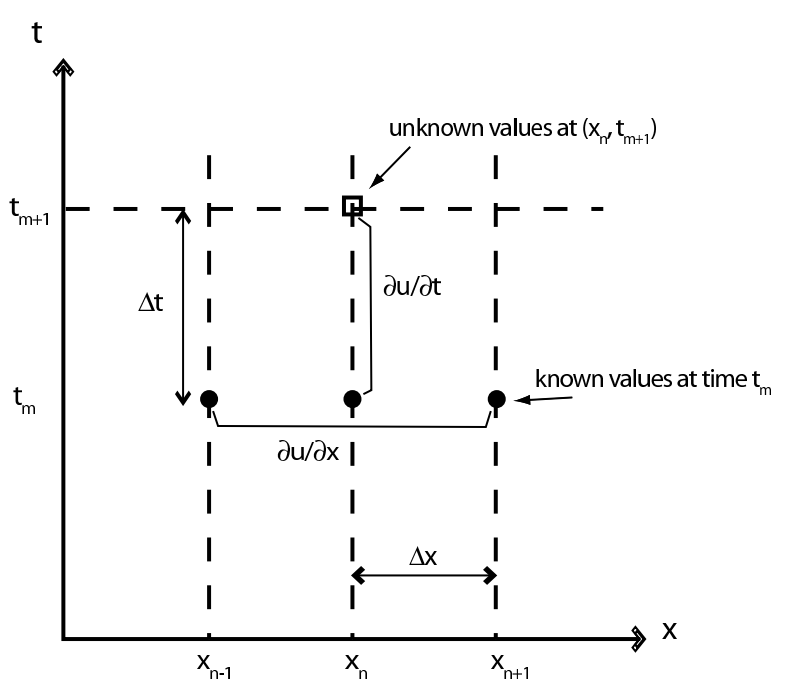

FIGURE 6.2 Finite difference modelling method. The method approximates partial derivatives $\left(\frac{\partial u}{\partial x}, \frac{\partial u}{\partial t}\right)$ by discrete differences at two different places with a finite distance $\Delta x, \Delta t$ to each other.

As shown in chapter 3.1 ( $c f$. equation 3.7), the anisotropic stress-displacement relationship is:

$$
\sigma_{i j}=C_{i j k l} \frac{1}{2}\left(\frac{\partial u_{k}}{\partial n_{l}}+\frac{\partial u_{l}}{\partial n_{l}}\right)
$$

where $C_{i j k l}$ is the fourth-order elastic tensor. Substituting equation 6.2 into equation 6.1 permits to write and solve the wave equation entirely in terms of displacement. This wave equation includes first- and second-order derivative (of time and space) and the solution depends on the elastic constants. To solve the wave equation in the finite difference method, the derivatives with respect to time are expressed by approximations:

$$
\begin{aligned}
\frac{\partial u}{\partial t} & =\frac{u_{n+1}-u_{n-1}}{2 \Delta t} \\
\frac{\partial^{2} u}{\partial t^{2}} & =\frac{u_{n+1}-2 u_{n}+u_{n-1}}{\Delta t^{2}}
\end{aligned}
$$

The spatial derivatives are obtained in the same way as in the example above (equations 6.3 and 6.4). The equation can then be solved explicitly for the displacements $u_{n+1}$ in terms of the previous displacements at time steps $n$ and $n-1$ (figure 6.2). The results provide the second-order finite difference solution to the anisotropic wave equation for displacement.

A numerical mesh with a finite number of points describes the model space, i.e. the simulated medium the wave is travelling through. Elastic constants can be varied at any point in the model and, depending on sufficiently fine discretisation, the model can be very accurate. 


\section{Numerical restrictions}

The numerical stability depends on the size of the steps in time $\Delta t$ and space $\Delta x, \Delta y, \Delta z$ used in the model. Therefore, computational artefacts can be avoided by choosing appropriate model parameters.

A common problem of waveform modelling is grid dispersion. This artefact occurs whenever a continuous medium is approximated by a discrete grid. Even if the model includes homogenous velocity with no dispersion, the dispersion of the calculated seismic responses appear as "normal" variation of velocity with frequency, that is, the higher signal frequencies are delayed relative to the lower signal frequencies and substantial "tailing" of the signal arises. The effect of dispersion increases for coarser grids and larger grid intervals. As a rule of thumb to minimise dispersion, the grid should consist of more than ten grid point per wavelength, considering the minimum velocity [Kelly et al., 1976].

Another criterion for numerical stability is the appropriate choice of time steps $\Delta t$. If $\Delta t$ is too large in an attempt to save computation time, the faster velocities make the discretised wavefield aliased in time. In order to minimise this effect, $\Delta t$ should follow the condition [Kelly et al., 1976]:

$$
\begin{aligned}
\Delta t & \leq \frac{\Delta x}{\sqrt{\mu / \rho+(\lambda+2 \mu) / \rho}} \\
\Delta t & \leq \frac{\Delta x}{\sqrt{v_{S}^{2}+v_{P}^{2}}}
\end{aligned}
$$

where $v_{S}$ and $v_{P}$ are the maximum velocities defined by the stiffness tensors used in the model.

A general rule implies that the higher the frequency and the lower the smallest velocity, the smaller the required sampling, both in time and space. To avoid aliasing, the grid sampling has to follow the relation:

$$
\Delta x \leq \frac{v_{\min }}{2 f_{\max }}
$$

\section{Boundary conditions}

The lateral and vertical extension of the finite difference model space is limited by the available computer memory (see next section). This restriction in space introduces artificial boundaries non-existent in nature, which produce undesired edge reflections.

Different approaches are available to mitigate this problem. The simplest approach is to increase the model size and neglect the boundary areas. However, this is limited due to numerical restriction ( $c f$. above). Other solutions give special properties to the boundaries, in order to minimise undesired effects. 
- The absorbing boundary [Higdon, 1991] defines the displacement on the boundary as a function of the neighbouring displacements, times and velocities. It is effective near free surfaces.

$$
u_{t+1}(u, v)=-q_{x}(v) u_{t+1,2}-q_{t}(v) u_{t, 1}-q_{t x}(v) u_{t, 2},
$$

where $q_{x}, q_{t}$ and $q_{t x}$ are scalar constants, defined by Higdon [1991].

- The reflecting boundary sets the displacement at the boundary equal to zero.

- The stress-free boundary sets the surface-perpendicular displacement gradient to zero. Free surfaces are always stress free. In the way this boundary condition is applied in the code used in this study, it is stable for $v_{S} / v_{P}>0.25$ [Boyd, 2006].

- An additional boundary condition exists for the bottom edge of the model, where nonvertical wavefields generate additional artefacts. But a boundary condition to minimise additional wavefields created on the vertical boundary towards the source is not implemented in Boyd's code [Boyd, 2006]. The specific implementation of boundary conditions in the code might be the reason for its inability to allow wave incidence from the right-hand side into the model space.

\section{Technical details}

For this work modelling was performed on a Linux system, with a dual core $3.2 \mathrm{GHz}$ processor and $4 \mathrm{~Gb}$ RAM. The maximum possible model space to use on this machine comprises 400 nodes in the $x$-direction, one node in the y-direction and 300 nodes in the $z$-direction, before Matlab ${ }^{\circledR}$ reported memory problems. Processing time for the largest models takes about 20 minutes.

\section{Assumptions}

It needs to be emphasised that assumptions always underlie models. The main assumption of the finite difference modelling applied here is a constant density throughout the model space. This is needed to write the above equations as functions of the spatially varying $\mathrm{P}$ - and S-wave velocities. The input wave is chosen as a Gaussian waveform of any given frequency.

\subsubsection{Modelling the central North Island}

The geometry of the complex setting of the Hikurangi subduction zone cannot be sufficiently described by layered structure, as was shown in the previous section (6.1) and appendix G. Forward modelling using the finite difference method provides an adequate means to model full waveform propagation through the complex subduction zone setting with domains of 


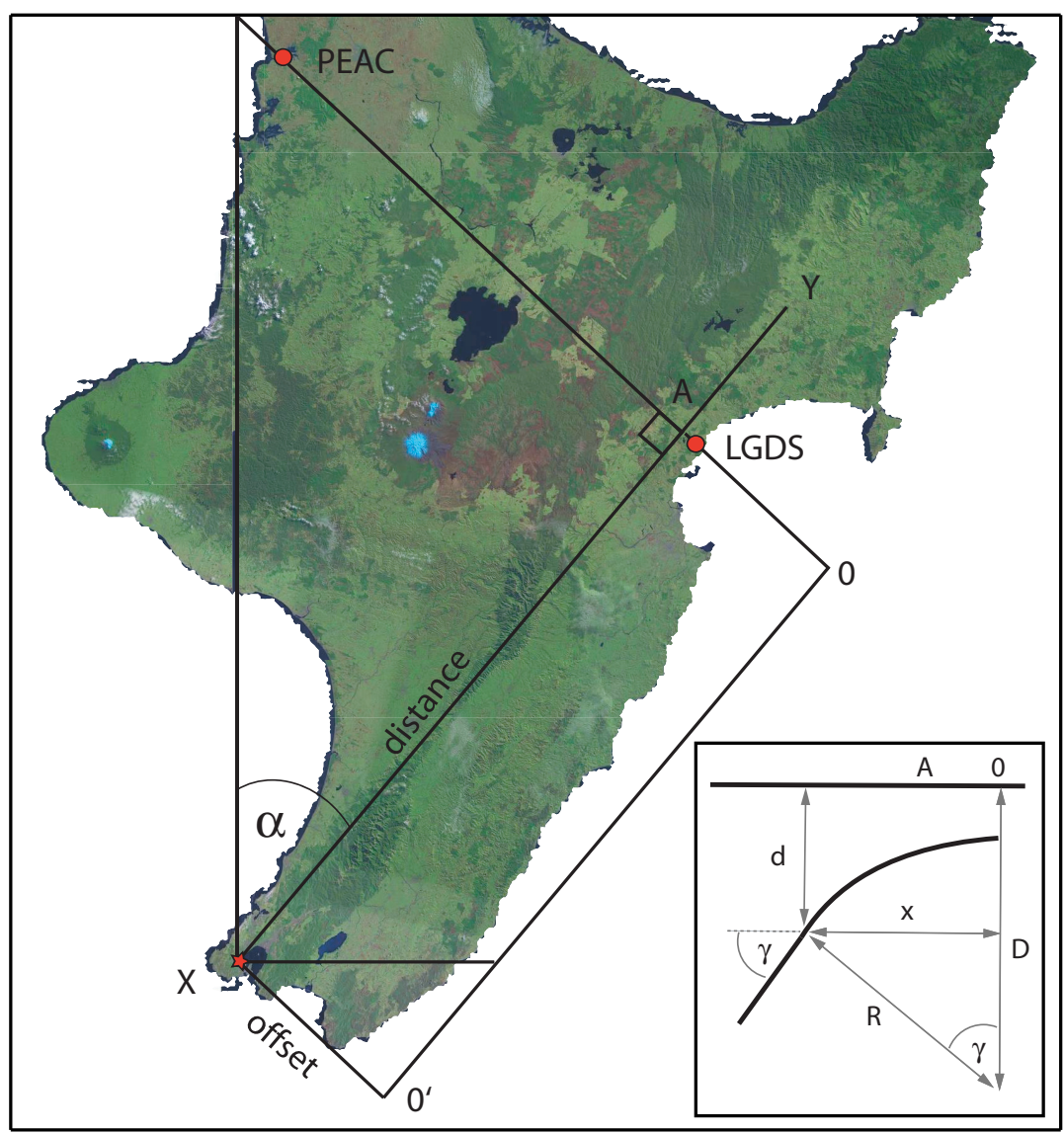

FIGURE 6.3 Geometry of the Hikurangi subduction zone model space after Ansell \& Bannister [1996]. Inset shows cross section along the CNIPSE line. " $X$ " is null point set in Wellington. For further description of parameters refer to text and table 6.1.

different elastic properties. The main difference between the analytical models and the numerical models lies in the way the actual splitting parameters are derived. For analytical models, theoretical splitting parameters are calculated from the velocities derived from the elastic tensor. With numerical models, splitting parameters can be measured on the synthetic waveforms in exactly the same way as with real data.

The model setup used in this study is based on the subduction geometry of the Hikurangi subduction zone for a cross-section along the (W)CNIPSE line (figures 6.3 and 6.4). The top and base of the subducting slab are included as a circle segments at shallow depths and as straight lines at depths greater than $d$. Table 6.1 gives the exact dimensions for modelling the slab. The values to describe the geometry of the slab are calculated based on equations defined by Ansell \& Bannister [1996] for the cross-section considered here. Furthermore, there are variable parameters describing the model space, such as the thickness of the slab and a parameter defining the location of the arc, i.e. the boundary between the forearc and the backarc. 
TABLE 6.1 Parameters used for model setup as shown in figure 6.3 and figure 6.4.

\begin{tabular}{|l|c|c|}
\hline centre of circle describing upper slab & $\mathrm{D}$ & $257.7 \mathrm{~km}$ \\
radius of circle describing upper slab & $\mathrm{R}$ & $244.8 \mathrm{~km}$ \\
distance from centre of circle to right model boundary & $\mathrm{b}$ & $50 \mathrm{~km}$ \\
depth to start lower (straight) slab & $\mathrm{d}$ & $150 \mathrm{~km}$ \\
dip of slab at intersection upper-lower & $\gamma$ & $63^{\circ}$ \\
offset of radius (as defined in Ansell \& Bannister [1996] & offset & $66.7 \mathrm{~km}$ \\
distance of cross section from Wellington & distance & $292.5 \mathrm{~km}$ \\
slab thickness & $\mathrm{s}$ & $50 \mathrm{~km}$ \\
strike of slab & $\alpha$ & $30^{\circ}$ \\
\hline
\end{tabular}

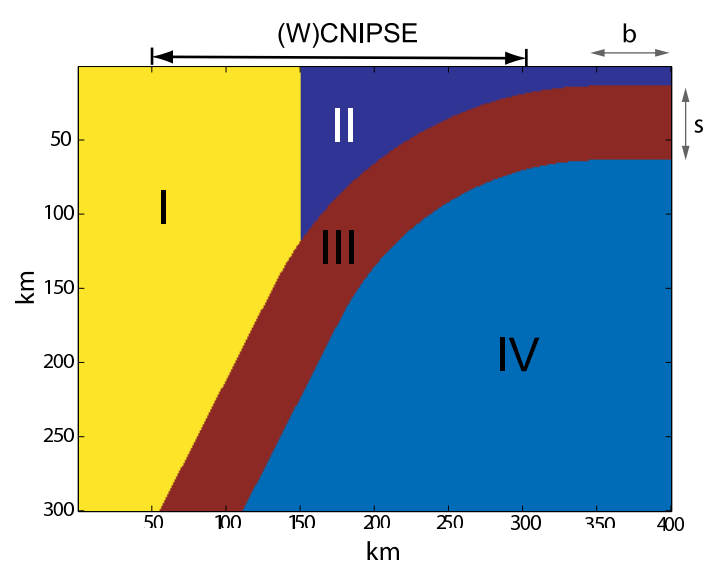

FIGURE 6.4 2D geometry for finite difference modelling. The four different (anisotropic) domains used in the modelling - backarc (region I), mantle wedge (region II), slab (region III) and subslab (region IV) - defined by full elastic tensors. " $s$ " is the slab thickness and " $b$ " is the length of the horizontal part of the slab.

The described geometry divides the model space into four domains (figure 6.4):

I Backarc

II Mantle wedge (Forearc)

III Slab

IV Sub-slab

All domains can be described with their own elastic properties defined by the full elastic tensors. The input for all the models are plane S-waves.

Table 6.2 gives an overview of all the models run. The elastic properties for the four domains vary between isotropic olivine, anisotropic olivine (describing high anisotropy) and the xenolith found in North Island near Raglan (describing average 3.5\% anisotropy) [Duclos, 2005] (cf. table A.1). Even higher anisotropy than olivine is described by a synthetic tensor with a slow axis of symmetry. Symmetry axes are kept horizontal for most of the models, as modelling with horizontal symmetry axis matched data well (cf. section G.1) and dipping symmetry axes did not improve the fit for central North Island (cf. section G.2).

Boundary conditions chosen for the modelling are a free boundary on the boundary facing towards the event backazimuth and an absorbing boundary on the opposite boundary. The cross-section modelled is extended on both ends of the original line (about $245 \mathrm{~km}$ long) to a total of $400 \mathrm{~km}$. Based the distribution of the events used for the original shear-wave 
TABLE 6.2 Finite difference models. Abbreviations stand for elastic properties: Iso=isotropic olivine, oliv=pure olivine, NIX=North Island Xenolith [Duclos, 2005], with fast polarisation and dip of symmetry axis given in brackets. All models use a constant density of $3.3 \mathrm{~kg} / \mathrm{m}^{3}$, a wave period of $4 \mathrm{~s}$, a sampling rate of $0.07 \mathrm{~s}$ and a horizontal slowness of 0 . All models starting from model All11 are additionally run with slownesses of 4e-5, for easterly and westerly incidence.

\begin{tabular}{|c|c|c|c|c|c|c|c|c|}
\hline \multirow{2}{*}{$\begin{array}{l}\text { Model } \\
\text { name }\end{array}$} & \multicolumn{4}{|c|}{ Region } & \multicolumn{3}{|c|}{ Grid points } & \multirow{2}{*}{$\begin{array}{c}\text { Grid } \\
\text { sampling }\end{array}$} \\
\hline & I & II & III & IV & $\mathbf{x}$ & $y$ & $\mathbf{z}$ & \\
\hline All8 & iso & NIX $(30,0)$ & iso & $\operatorname{NIX}(30,0)$ & 239 & 2 & 200 & $1.5 \mathrm{~km}$ \\
\hline All9 & iso & NIX $(30,0)$ & oliv $(30,0)$ & $\operatorname{NIX}(30,0)$ & 239 & 2 & 200 & $1.5 \mathrm{~km}$ \\
\hline All10 & iso & oliv $(30,0)$ & oliv $(30,0)$ & $\operatorname{NIX}(30,0)$ & 239 & 2 & 200 & $1.5 \mathrm{~km}$ \\
\hline All11 & iso & oliv $(0,0)$ & oliv $(0,0)$ & $\operatorname{NIX}(0,0)$ & 400 & 1 & 300 & $1 \mathrm{~km}$ \\
\hline All12 & iso & $\operatorname{NIX}(0,0)$ & oliv $(0,0)$ & $\operatorname{NIX}(0,0)$ & 400 & 1 & 300 & $1 \mathrm{~km}$ \\
\hline All13 & iso & oliv $(0,0)$ & iso $(0,0)$ & $\operatorname{NIX}(0,0)$ & 400 & 1 & 300 & $1 \mathrm{~km}$ \\
\hline All14 & iso & iso & oliv $(0,0)$ & iso & 400 & 1 & 300 & $1 \mathrm{~km}$ \\
\hline All15 & iso & iso & oliv $(0,0)$ & $\operatorname{NIX}(90,0)$ & 400 & 1 & 300 & $1 \mathrm{~km}$ \\
\hline All16 & iso & iso & oliv $(90,27)$ & iso & 400 & 1 & 300 & $1 \mathrm{~km}$ \\
\hline All17 & iso & iso & oliv $(90,53)$ & iso & 400 & 1 & 300 & $1 \mathrm{~km}$ \\
\hline All18 & iso & $\operatorname{NIX}(0,0)$ & oliv $(90,63)$ & $\operatorname{NIX}(0,0)$ & 400 & 1 & 300 & $1 \mathrm{~km}$ \\
\hline All19 & iso & $\operatorname{NIX}(0,0)$ & oliv $(90,30)$ & $\operatorname{NIX}(0,0)$ & 400 & 1 & 300 & $1 \mathrm{~km}$ \\
\hline All20 & iso & oliv $(0,0)$ & oliv $(90,30)$ & $\operatorname{NIX}(0,0)$ & 400 & 1 & 300 & $1 \mathrm{~km}$ \\
\hline All21 & iso & oliv $(0,90)$ & iso & $\operatorname{NIX}(0,0)$ & 400 & 1 & 300 & $1 \mathrm{~km}$ \\
\hline
\end{tabular}

splitting measurements (figure 5.4), all models are run for wave incidences from the right and the left into the model space. This corresponds to events arriving from northwesterly and southeasterly backazimuths and approximates the majority of the data (figures 6.5 and 6.6). It was not attempted to model individual events. Moreover, ScS events are not considered in the modelling because of their higher frequency content and northerly backazimuths. The incoming polarisation was set to be $30^{\circ}$ from the prevailing fast direction in the model to prevent null measurements arising from the proximity of fast and incoming polarisation.

There were some problems with the code, which did not allow wavefronts to enter the model space from the right (cf. section 6.2.1). Therefore, to model events from easterly backazimuths the model space is mirrored, wavefronts are propagated from the left and results are mirrored again.

The preferred model was determined by visual inspection of the match to the teleseismic splitting measurements from section 5.4. Figures 6.6(a) and 6.6(b) show inferior models, which helped to confine the preferred model. Synthetic splitting parameters are determined from automated shear-wave splitting measurements [Teanby et al., 2004] (cf. section 5.3) on the synthetic waveforms (figure 6.6, top). In the following they are referred to as "synthetic" data. For the direct comparison of splitting parameters from the synthetic waveforms to the ones from real data (in the following referred to as "real" data), the fast polarisation results have to be rotated from the model coordinate system, which aligns with the CNIPSE line, into geographic coordinates. This is done by rotating the synthetic fast polarisations 


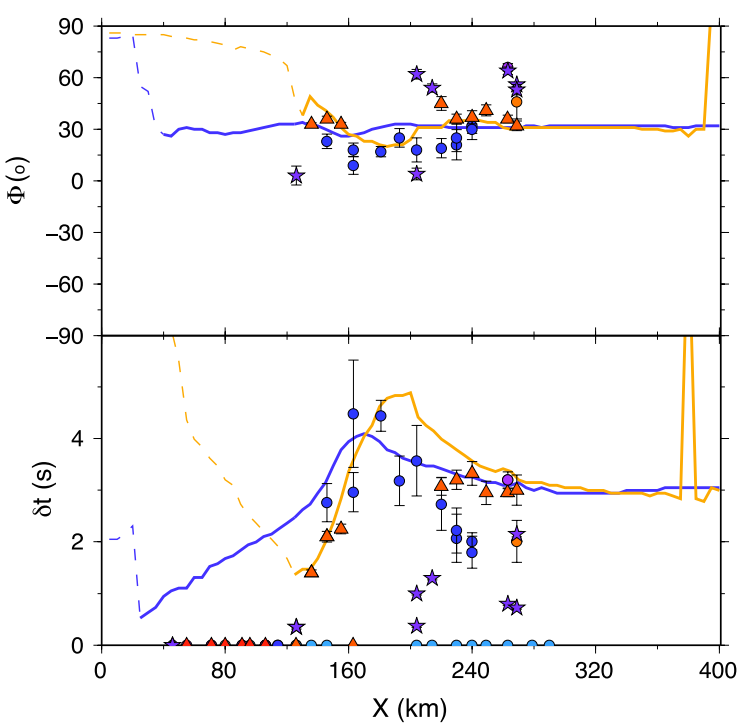

(a) Model All11

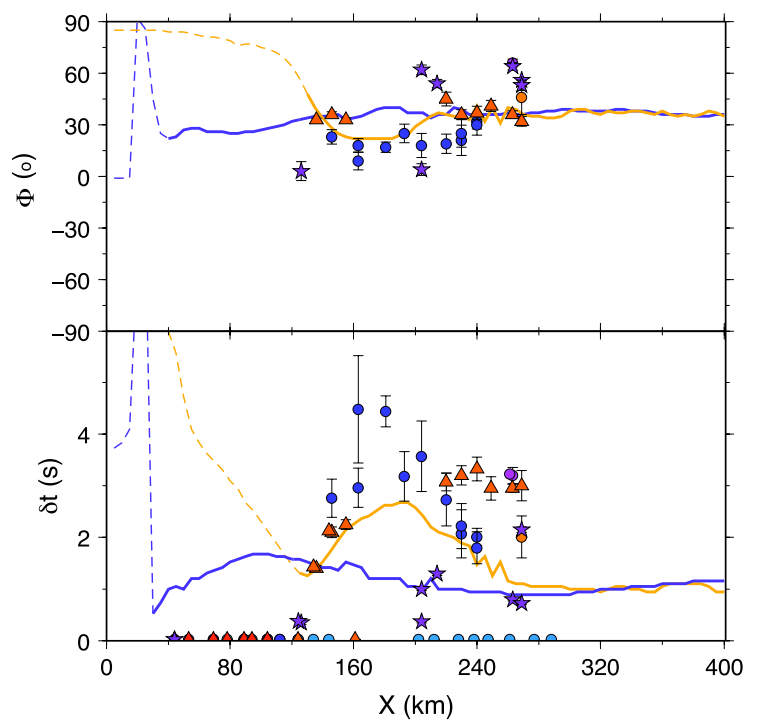

(b) Model All14

FIGURE 6.5 Results for two inferior finite difference models. Shear-wave splitting parameters for models (line) compared to real data (circles: SKS-, triangle: S-, stars: ScS-phases). Colours indicate different backazimuths (incoming polarisation), blue=east, orange=west. Where no reliable shear-wave splitting parameter could be measured, e.g. for null measurements, the lines are dashed (cf. text for identification of null measurements).

by $30^{\circ}$.

The model results shown in figure 6.5 show several characteristics that do not fit the real data. Model All11 (figure 6.6(a)) includes high anisotropy in the wedge and the slab. This results in delay times for easterly arrivals (blue) that decrease over a wider range than observed in the data. Moreover, delay times for westerly arrivals (orange) become larger than the already high delay times observed for easterly arrivals. In model All14 (figure 6.6(b)), on the other hand, the only anisotropic region is the slab. In this model, delay times have the expected smooth variations for westerly arrivals, even though the overall magnitude is too small. But events from the east stay almost constant in delay times, with a slight decrease around kilometre 120, where nulls are observed in the real data. In both models (figures 6.6(a) and 6.6(b)), the fast polarisations for westerly arrivals vary towards more northerly directions compared to the average fast polarisation of about $30^{\circ}$. This variation, however, is expected for easterly arrivals. Furthermore, the region where nulls are observed is narrower for easterly arrival (starting around kilometre 40) than observed in the data (starting around kilometre 120). Those mismatches distinguish these models from the preferred model (figure 6.6). The distinct changes in delay times over a narrow distance constrain a lack of anisotropy in the slab and require high anisotropy in the wedge.

In the preferred model, the synthetic waveforms (figure 6.6, top) exhibit a distinct change between kilometre 120 and 160. East of kilometre 160, the two S-waves (red and blue) are split, with the blue waveform arriving earlier than the red. West of kilometre 120, both 


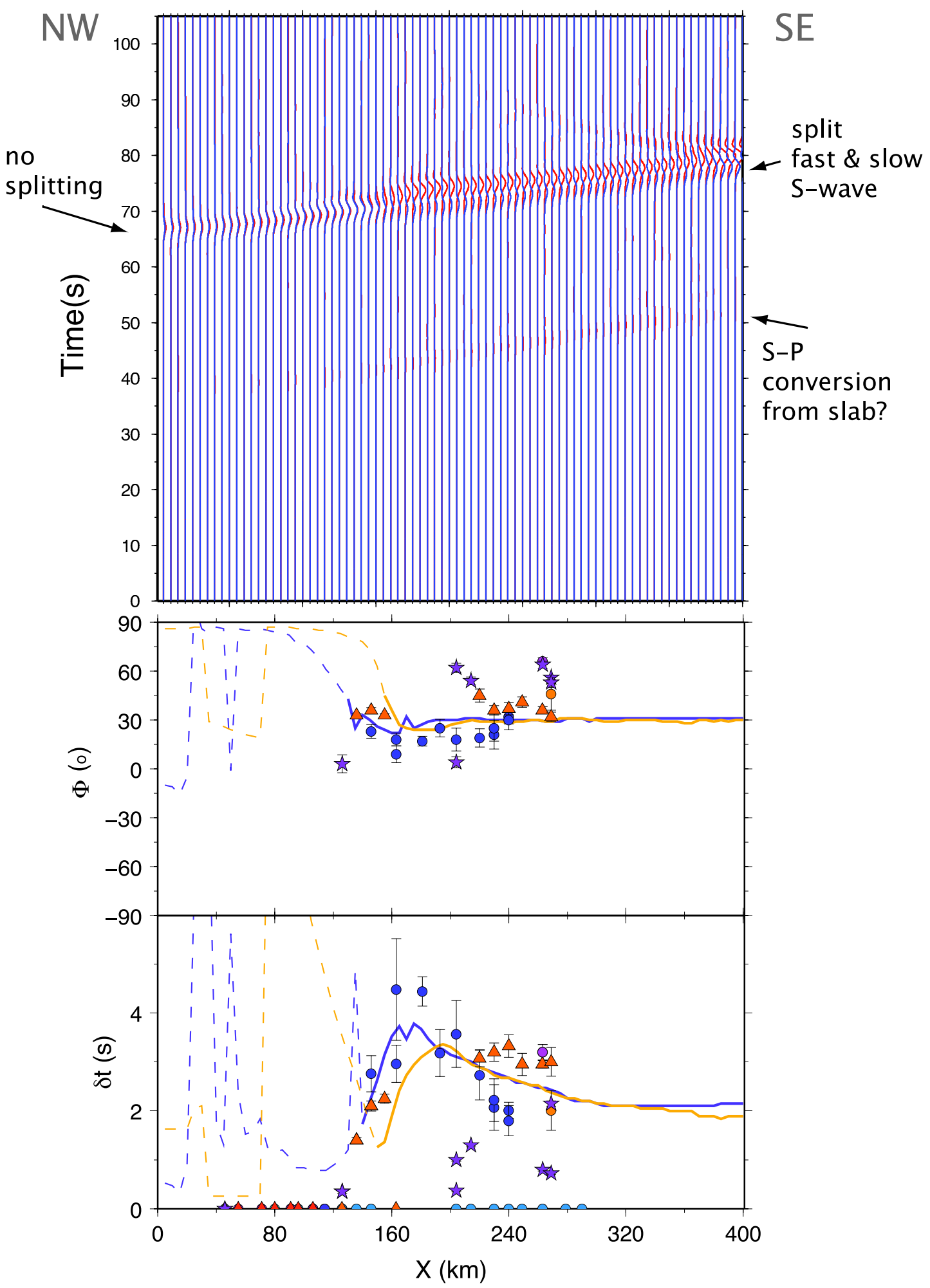

FIGURE 6.6 Results for preferred finite difference model (model All21, table 6.2) Top: Synthetic waveforms of the two horizontal components (blue=parallel to model, red=perpendicular) in model reference frame, used for shear-wave splitting. Bottom: Shear-wave splitting parameters from model (line) compared to real data (circles: SKS-, triangle: S-, stars: ScS-phases). Colours indicate different backazimuth (incoming polarisation), blue=east, orange=west. Where no reliable shear-wave splitting parameter could be measured, e.g. for null measurements, the lines are dashed (see text for identification of null measurements). 
components match and arrive at the same time, i.e. no splitting is apparent. Additionally, a faint earlier arrival appears around $40-50 \mathrm{~s}$. As it is faster and less pronounced than the S-waves, it could be a S-P conversion from the slab.

Synthetic splitting measurements from the preferred model (figure 6.6) retrieve no stable splitting parameters up to kilometre 120. Measured fast polarisations tend to be close to the incoming polarisation (here $90^{\circ}$ in the geographic coordinate system) or normal to it (i.e. $0^{\circ}$ ) and delay times are either close to the fixed upper limit or close to zero. This identifies them as null measurements ( $c f$. section 5.3), showing no apparent splitting. This is consistent with the observed waveforms (figure 6.6, top). The location of the area of null measurements in the synthetic data, indicted by the dashed lines, coincides with the area of only null measurement in the real data (figure 6.6, bottom).

The real data set does not exhibit much variation in fast polarisations. Especially for easterly and westerly incoming polarisations, fast polarisations are mainly distributed around $\Phi=30^{\circ}$. Fast polarisations gained from westerly arrivals tend to more westerly, and easterly arrivals to more northerly orientations. Modelling results give consistent fast polarisations of $30^{\circ}$, as expected for the constant fast orientations put into the model. However, around kilometre 160-170, fast polarisations decrease slightly, before increasing around the boundary to the isotropic region. This matches with the decrease in the easterly real data, followed by an increase. The variations for westerly real data are not matched.

Variations in delay times are more pronounced than in fast polarisations and the differences for opposed incidence angles are more distinct. The splitting parameters from the modelling match the characteristic higher delay times for easterly arrivals compared to westerly in the region around kilometre 160. The peak for easterly delay times is shifted to the west relative to the one for westerly delays. The locations of the synthetic and real data peak match for easterly data. For westerly arrivals, the location of the peak cannot be determined in the real data, as measurements are missing between kilometre 160 and 220. For both incidence angles, the rapid decrease in delay times between kilometre 120 and 160 is fitted well by the synthetic data.

\subsection{Discussion and Conclusions}

The methods to model the anisotropic structure of central North Island introduced in this chapter evolve from a simple analytical two-layer model with horizontal symmetry axis over two-layered models with dipping symmetry axes to a finite difference model with full wave propagation. This evolution helped to restrict the possibilities of input parameters for the final forward finite difference model.

The best-fit two-layer models require only small differences in the fast polarisations of the two layers. This implies that a single layer with $\Phi=30-40^{\circ}$ can adequately fit the data. 
The two-layer modelling results are comparable to models determined for southern North Island. Gledhill \& Stuart [1996] find an upper layer of $\Phi=50^{\circ}$ with $\delta t=0.6 \mathrm{~s}$ and a lower layer of $\Phi=10^{\circ}$ with $1.6 \mathrm{~s}$ delay time as the best-fit two-layer model for SKS splitting data from an array in southern North Island. Marson-Pidgeon \& Savage [2004] get $\Phi=60^{\circ}$ with $\delta t=0.4 \mathrm{~s}$ and $\Phi=20^{\circ}$ with $1 \mathrm{~s}$ delay time for the upper and lower layer below station SNZO. They also get no significantly improved fit with introduction of dipping symmetry axes.

The finer the partitioning of data for the models gets, the less data are included in the modelling and the less the results are constrained. The majority of the data is well fitted by the most general, single layer model with horizontal symmetry axis. The division into the three regions slightly increases the fit and supports the theory of the different anisotropic regions. It singles out the CVR as a more complex region and western North Island as apparently isotropic.

The separation into different phases in both two-layer approaches impairs the data fit and no consistent model can be found. This is mainly caused by the lack of S- and ScS-phase data and could be more approachable for an extended data set. However, the difference between the regions seems to have more influence on the data than the difference between phases. The final combined models do not have enough data to achieve a good and unique fit. Due to the complex subduction zone setting, it is not surprising that models with two horizontal layers are not sufficient to explain observations. The difficulty of finding a consistently good two-layer model for both fast polarisation and delay time was already pointed out by Marson-Pidgeon \& Savage [2004].

The implementation of a realistic geometry in the finite difference method improves the model fit considerably. Overall, all large-scale characteristic of the measured splitting parameters are matched by the model results. This applies especially to the high delay times in the CVR and the rapid decrease in delay times to the west of the CVR. The preferred model consists of an isotropic region under western North Island, a highly anisotropic wedge, modelled as pure olivine, with trench-parallel fast orientation, an isotropic slab and a moderately anisotropic sub-slab. This verifies the interpretation suggested in chapter 5 for central North Island (see above). The model negates the possibility of anisotropy in the slab that was suggested in chapter 5 and distinguishes the central North Island from the lower North Island, where slab anisotropy is proposed. It agrees with results from Matcham et al. [2000], who found a decrease in anisotropy from southern (4.4\%) to central North Island. Introduction of slab anisotropy in the models widens the region over which delay times decay and increases delay times for westerly backazimuths and therefore diminishes the fit to observations (figure 6.5). However, the preferred model is non-unique and other solutions are possible.

Small-scale variations are not fitted by the model yet. This is due to the coarseness of the applied model setup. But the method provides the potential to include more detailed, even three-dimensional structure into the model. Therefore, actual results from other North 
Island studies, e.g. structure determined from receiver functions, tomography and active source surveys, and percentage anisotropy from local shear-wave splitting could be implemented. However, this implementation is beyond the scope of this study, but is suggested as an incentive for a future study. 


\section{Chapter 7}

\section{Modelling small-scale anisotropy}

This chapter focuses on modelling anisotropy at different scale lengths and the variation of shear-wave splitting observations with different frequencies. As mentioned before, the occurrence of anisotropy depends on scale length, i.e. the relative size of the wavelength compared to the size of the heterogeneities causing the anisotropy. Anisotropy only occurs if the heterogeneities are much smaller than the wavelength. As a rule of thumb, anisotropy is only apparent to wavelengths of eight to ten times the size of the heterogeneities [Lynn, 2004]. For smaller wavelengths, scattering, dispersion and attenuation take over.

Because of the scale dependent relation, frequency dependent anisotropy becomes important in material with aligned cracks or inclusions or in periodically thinly layered materials. The anisotropic strength depends on the size and spacing of the heterogeneities. Those parameters also define for which frequencies the medium appears anisotropic. In general, longer wavelengths sample anisotropy caused by larger structures. Exploration seismic studies relate frequency dependent anisotropy mainly to seismic scattering by heterogeneities or to fluid flow in fractured porous rock.

Most of the theoretical and experimental studies done on crack anisotropy roots in the oil exploration industry and active seismic acquisition [Helbig \& Thomsen, 2005]. Therefore, the materials most studied are layered sediments, such as sandstone, shale, limestone, gypsum, and wavelengths considered are ultrasonic (from laboratory work) to high frequency (frequency range for active seismic covers from about $10^{1}$ to $10^{2} \mathrm{~Hz}$ ). Typical depth ranges are the upper few hundred metres to kilometres of the Earth. Originally, the work was solely based on P-waves, but S-waves are becoming increasingly important in the exploration industry, as they are more sensitive to anisotropy [e.g. Winterstein, 1992; Lynn, 2004].

The following sections will introduce the basics of calculating elastic properties due to periodic thin layering (PTL) [Backus, 1962] and shape-preferred orientation (SPO) of inclusions. Theoretical approaches to shape-preferred orientation anisotropy were developed by Hudson [1981] and Thomsen [1995] for the upper and lower frequency limits and later extended into the frequency dependent intermediate frequency range by Chapman et al. 
[2002]. All three theories are summarised in the following.

\subsection{Periodic thin layering}

The theory to calculate the elastic tensor and therefore anisotropy due to periodic thin layering (PTL) of more than two isotropic or transversely isotropic materials with different properties was first introduced by Backus [1962]. This chapter presents a summary of the mathematical background as it is used in this work.

The following assumptions underlie the calcula-

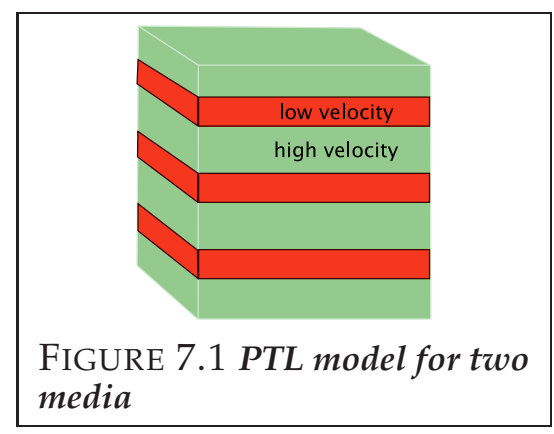
tions:

- The wavelength is much larger than the thickness of the layers (about eight times larger) [Carcione, 2007].

- The single layers are (transversely) isotropic and linear elastic.

- The layered medium itself is homogeneously transversely isotropic with elastic coefficients algebraically calculated from the elastic parameters of the original media. Its density is the weighted average density of the original media.

The components of the anisotropic elastic tensor for the averaged medium, often referred to as the Backus average [Backus, 1962], are calculated from the elastic parameters of the hexagonal tensors of the single layers (Love parameters a, b, c, 1, m, f; $c f$. section 3.1):

$$
\begin{aligned}
A & =\left\langle a-f^{2} / c\right\rangle+\langle 1 / c\rangle^{-1}\langle f / c\rangle^{2} \\
B & =\left\langle b-f^{2} / c\right\rangle+\langle 1 / c\rangle^{-1}\langle f / c\rangle^{2} \\
C & =\langle 1 / c\rangle^{-1} \\
F & =\langle 1 / c\rangle^{-1}\langle f / c\rangle \\
L & =\langle 1 / l\rangle^{-1} \\
M & =\langle m\rangle
\end{aligned}
$$

The brackets indicate averages of the elastic constants of the layers weighted by their volumetric proportion, defined as:

$$
\langle a\rangle=\sum_{n=1}^{N} p_{n} a_{n},
$$

where $p_{n}$ is the proportion of material $n$. 
For example, in a medium consisting of two layers with layer 1 taking up a fraction $p_{1}$ and layer 2 a fraction $p_{2}$, with $p_{1}+p_{2}=1$, the resulting parameters $M$ and $L$ are calculated as the harmonic and arithmetic average, respectively:

$$
\begin{aligned}
M & =p_{1} m_{1}+p_{2} m_{2} \\
L & =p_{1} / l_{1}+p_{2} / l_{2}
\end{aligned}
$$

This means that in periodic thin layered media $L$ is always smaller than $M$, unless all shear stiffnesses are equal. Then $L$ is equal to $M$ and the compound medium is isotropic.

\subsection{Oriented cracks and inclusions}

Several authors published expressions to calculate overall elastic constants for media containing oriented inclusions like cracks or fractures (shape-preferred orientation) using effective medium theory. The first one was Eshelby [1957] and most subsequent theories are based on his work. The theories of Hudson [1981], Tandon \& Weng [1983] and Thomsen [1995] are introduced here in detail.

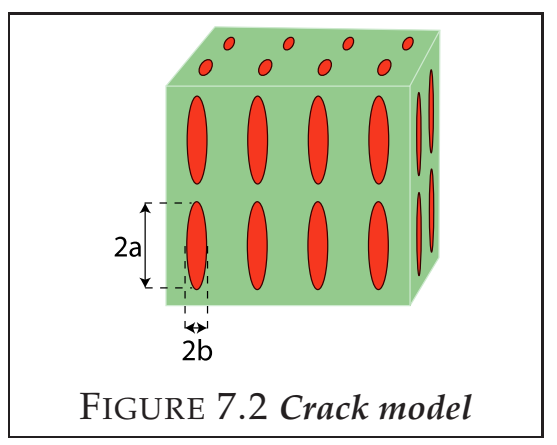

Laboratory studies allow one to compare analytically derived elastic properties with real measurements. The main problem of these comparisons is the scale length. Laboratory samples are small and frequencies used to measure their properties are too high to convincingly model conditions observed with seismic frequencies in the Earth. Nevertheless, studies show surprisingly similar properties and behaviour [e.g. Rathore et al., 1995]. The main problem of analytical calculations is that they rely on a broad range of parameters to describe the media. Especially for deeper structures in the Earth, little is known about specific properties of the material.

Analogous to the crystal structures, oriented, ordered sets of layers, cracks or fractures can be described by symmetry systems [Winterstein, 1990] (table 7.1).

A common parameter for theories related to crack anisotropy is the crack density $\varepsilon$ [Hudson, 1980; Crampin, 1993]. It is defined as the product of the number density of cracks $v$ (number of cracks per cubic meter) and the cube of the crack radius $a$ (for circular flat cracks):

$$
\varepsilon=v a^{3}=\frac{N}{V} a^{3},
$$

where $N$ is the total number of cracks per volume $V$.

Cracks are typically modelled as spheroids, i.e. degenerated ellipsoids with two axes of equal length. This is justified, as pore compressibility of an ellipsoid mainly depends on the 
TABLE 7.1 Symmetries of ordered inclusions

\begin{tabular}{|c|c|}
\hline Configuration & Symmetry \\
\hline \hline 1 set of fractures & transverse isotropic \\
& hexagonal \\
& orthorhombic \\
2 sets of fractures at right angles, different properties & orthorhombic \\
2 sets of fractures at arbitrary angle, same properties & tetragonal \\
2 sets of fractures at right angles, same properties & monoclinic \\
2 or more sets, arbitrary angles and properties & \\
\hline
\end{tabular}

ratio of the minimum to the intermediate axis [Jaeger et al., 2007]. The aspect ratio $\alpha$ for spheroids is defined as the length of the unequal axis to the length of one of the equal axes (figure 7.2). Spheroids with $\alpha>1$ are called prolate and for $\alpha \rightarrow \infty$ they become needlelike cylinders. Spheroids with $\alpha<1$ are called oblate. For $\alpha \rightarrow 0$ oblate spheroids become "penny-shaped" cracks.

Most of the models described below are valid for penny-shaped cracks ( $\alpha \simeq 0.001)$ [Barton, 2007] and low crack density $(\varepsilon<0.1)$ [Hudson, 1981].

\subsubsection{Hudson theory}

The theory of Hudson [1981] derives material properties due to circular inclusions for the high frequency approximation $(k a / 2 \ll 1)$ described by the shear-wave number $k$. The calculations are underlain by the following assumptions:

- Cracks are randomly distributed and their concentration and aspect ratio are small.

- Wavelengths are large compared to crack size and spacing.

- The inclusions are isolated in terms of flow. Therefore, the approaches are only valid for high frequencies such as laboratory conditions, where there is no time for pressure and fluid to equilibrate during the passing through of a wave.

- Cracks are thin and circular (penny-shaped) and either empty or filled with solid or fluid. The elastic properties of both the inclusions $C_{i j}^{1}$ and the background medium $C_{i j}^{0}$ are isotropic.

Under these assumptions the effective medium appears transversely isotropic with symmetry axis in the $x_{3}$-direction. Its elastic tensor is the sum of the background elastic tensor $C^{0}$ and a correction term $C^{1}$ :

$$
C_{i j}=C_{i j}^{0}+C_{i j}^{1}
$$

For fluid filled cracks the elastic components of the correction factor $C^{1}$ can be written as:

$$
C_{44}=C_{55}=-\frac{16}{3}\left(v a^{3}\right) \mu\left(\frac{\lambda+2 \mu}{3 \lambda+4 \mu}\right)
$$


with all other components being zero.

Note that Hudson's [Hudson, 1981] original notation for $C_{44}$ and $C_{55}$ differs by a factor 2 from the ones given in chapter 3 , as he uses a different definition of the elastic tensor. Thus, the equations given here are modified from his original ones and corrected for the additional factor.

For dry cracks the elastic components of $C^{1}$ are defined as:

$$
\begin{aligned}
& C_{11}=C_{12}=C_{21}=C_{22}=-\frac{4}{3}\left(v a^{3}\right) \frac{\lambda^{2}(\lambda+2 \mu)}{\mu(\lambda+\mu)} \\
& C_{13}=C_{23}=C_{31}=C_{32}=-\frac{4}{3}\left(v a^{3}\right) \frac{\lambda(\lambda+2 \mu)^{2}}{\mu(\lambda+\mu)} \\
& C_{33}=-\frac{4}{3}\left(v a^{3}\right) \frac{(\lambda+2 \mu)^{3}}{\mu(\lambda+\mu)} \\
& C_{44}=C_{55}=-\frac{16}{3}\left(v a^{3}\right) \frac{\mu(\lambda+2 \mu)}{3 \lambda+4 \mu},
\end{aligned}
$$

with all other components being zero.

For ellipsoid cracks filled with weak material with the semi-axis $a, a$, and $b$ of the ellipsoid, the elastic components are defined as:

$$
\begin{aligned}
& C_{11}=C_{12}=C_{21}=C_{22}=-\frac{4}{3}\left(v a^{3}\right) \frac{\lambda^{2}(\lambda+2 \mu)}{\mu(\lambda+\mu)(1+K)} \\
& C_{13}=C_{23}=C_{31}=C_{32}=-\frac{4}{3}\left(v a^{3}\right) \frac{\lambda(\lambda+2 \mu)^{2}}{\mu(\lambda+\mu)(1+K)} \\
& C_{33}=-\frac{4}{3}\left(v a^{3}\right) \frac{(\lambda+2 \mu)^{3}}{\mu(\lambda+\mu)(1+K)} \\
& C_{44}=C_{55}=-\frac{16}{3}\left(v a^{3}\right) \frac{\mu(\lambda+2 \mu)}{(3 \lambda+4 \mu)(1+M)}
\end{aligned}
$$

Here, the parameters $M$ and $K$ are calculated from:

$$
\begin{aligned}
K & =\frac{a\left(\kappa^{\prime}+(4 / 3) \mu^{\prime}\right)}{\pi b \mu}\left(\frac{\lambda+2 \mu}{\lambda+\mu}\right) \\
M & =\frac{4}{\pi}\left(\frac{a \mu^{\prime}}{b \mu}\right)\left(\frac{\lambda+2 \mu}{3 \lambda+4 \mu}\right)
\end{aligned}
$$

where $\mu$ and $\kappa$ are the shear and bulk modulus of the background medium and $\mu^{\prime}$ and $\kappa^{\prime}$ are the elastic moduli of the inclusion.

\subsubsection{Tandon and Weng theory}

Another theory to calculate elastic parameters due to cracks or inclusions was developed by Tandon \& Weng [1983] for polymer composites. It was originally developed to investigate 
the dependence of elastic moduli of transversely isotropic composites on aspect ratios of aligned inclusions and considers pertubations in applied stress due to the inclusions. The Tandon and Weng theory allows high-velocity inclusions and high-volume fractions and has been used in several geophysical papers to calculate seismic anisotropy [e.g. Ayele et al., 2004; Kendall et al., 2006].

There is an extensive formula assembly to calculate the five independent elastic constants, given as the longitudinal Young's modulus $E_{11}$, the transverse Young's modulus $E_{22}$, the inplane shear modulus $\mu_{12}$, the out-of-plane shear modulus $\mu_{23}$ and the plain-strain bulk modulus $K_{23}$. Further, there is the Poisson ratio $\nu_{12}$, which depends on the other constants.

For inclusions aligned along the $x_{1}$-axis with an aspect ratio $\alpha$ and taking up a volume fraction $c$, the elastic parameters of the effective medium are calculated as:

$$
\begin{aligned}
\frac{E_{11}}{E_{0}} & =\frac{1}{1+c\left(A_{1}+2 \nu_{0} A_{2}\right) / A} \\
\frac{E_{22}}{E_{0}} & =\frac{1}{1+c\left(-2 \nu_{0} A_{3}+\left(1-\nu_{0}\right) A_{4}+\left(1+\nu_{0}\right) A_{5} A\right) / 2 A} \\
\frac{\mu_{12}}{\mu_{0}} & =1+\frac{c}{\frac{\mu_{0}}{\mu_{1}-\mu_{0}}+2(1-c) S_{1212}} \\
\frac{\mu_{23}}{\mu_{0}} & =1+\frac{c}{\frac{\mu_{0}}{\mu_{1}-\mu_{0}}+2(1-c) S_{2323}} \\
\frac{K_{23}}{K_{0}} & =\frac{\left(1+\nu_{0}\right)\left(1-2 \nu_{0}\right)}{1-\nu_{0}\left(1+2 \nu_{12}\right)+c\left(2\left(\nu_{12}-\nu_{0}\right) A_{3}+\left[1-\nu_{0}\left(1+2 \nu_{12}\right)\right] A_{4}\right) / A} \\
\nu_{12}^{2} & =\frac{E_{11}}{E_{22}}-\frac{E_{11}}{4}\left(\frac{1}{\mu_{23}}+\frac{1}{K_{23}}\right),
\end{aligned}
$$

where the index " 0 " denotes the properties of the matrix and " 1 " denotes the properties of the inclusion. The definitions of the terms $A, A_{1}-A_{5}$ are given in appendix F.1.

\subsubsection{Thomsen theory}

Based on the introduction of five new parameters to describe hexagonal anisotropy [Thomsen, 1986] ( $c f$. appendix F.2), Thomsen [1995] developed a new theory to calculate anisotropy due to cracks. In contrast to other theories, Thomsen's theory [Thomsen, 1995] is valid for brine pore fluids, substantial equant porosity and lower frequency bands (see below). It is therefore often referred to as the lower frequency limit for crack induced anisotropy. These inclusions make the theory suitable for applications to upper crust conditions.

The theory is based on the following assumptions:

- The matrix is isotropic and homogeneous.

- Cracks are small compared to the wavelength.

- The crack density is low and cracks are thin. 
- The resulting medium is transversely isotropic with the axis of symmetry perpendicular to the flat faces of the (elliptical) cracks.

- Fluid pressure equalises between cracks and the surrounding rock (limited to low frequencies).

This last point is the main difference between Thomsen's and Hudson's model. It results from the introduction of pore space, described by the porosity, to the matrix rock. In particular, Thomsen defines the concept of "equant" porosity, meaning that pore spaces are not "thin and flat" and do not have any particular fabric. This becomes important with the effect of fluid flow on anisotropy. Interesting implications of the theory appear for low frequencies, where the anisotropy is independent of aspect ratios and only dependent on crack density.

The existence of pore space surrounding the cracks means that the cracks are no longer isolated with respect to flow. The dynamic of the pore fluid under the influence of a seismic wave is described by the squirt (flow) model. The stress changes caused by a seismic wave travelling through a porous rock induce gradients in pore pressure. These pore pressure gradients influence the viscoelastic properties of the rock. At high frequencies, the gradients are unrelaxed and the elastic moduli will be stiffer than at lower frequencies. In the low frequency range, which includes the seismic frequency band, there is enough time between each peak of the wave amplitude for the fluid pressure to equilibrate between cracks and pores. The moderately high frequency band is appropriate for laboratory data in the ultrasonic band.

Thomsen defines the low frequency range as frequencies much less than the so-called squirt flow frequency. The squirt flow frequency is usually assumed to be between the sonic and ultrasonic frequency band, but depends on the details of the crack-pore microgeometry and the properties of the fluid. Mavko [1980] defines the squirt flow relaxation time as the ratio between the squared characteristic diffusion length and the diffusivity. Thomsen's theory also highlights that pore fluid affects anisotropy in a frequency dependent way. However, all calculations are independent of frequency.

The most important parameter in Thomsen's calculations [Thomsen, 1995] is the "fluid influence factor". For the low frequency range, it is defined as:

$$
D_{c i}=\left(1-\frac{K_{f}}{K_{s}}+\frac{K_{f}}{K^{*} \phi}\left(\left(1-\frac{K^{*}}{K_{s}}\right)+A_{c}\left(\nu^{*}\right) \eta_{c}\right)\right)^{-1}
$$

Here, $K_{f}$ is the bulk modulus (incompressibility) of fluid in cracks, $K_{s}$ is the bulk modulus of solid grains, $K^{*}$ is the bulk modulus of dry porous rock, $\phi$ is the total porosity including cracks and pores, $\eta_{c}$ is the fracture density and $A_{c}$ is a function of $\nu^{*}$ (Poisson ratio of dry porous rock) defined by:

$$
A_{c}\left(\nu^{*}\right)=\frac{16}{9} \frac{1-\nu^{* 2}}{1-2 \nu^{* 2}}
$$


The ratio $K_{f} / K_{s}$ has a strong influence on the P-wave velocity dependence on porosity, but less on the $\mathrm{S}$-wave velocity dependence. Another outcome of the Thomsen theory is significant angular dependence of attenuation and dispersion. However, at low, seismic frequencies dispersion is assumed to be small.

Rathore et al. [1995] compares laboratory measurements on synthetically cracked sandstone with calculated parameters from Hudson's and Thomsen's theory. For dry rocks, both theories fit the measurements well. For saturated samples, the fit is better with Thomsen's model.

\subsection{Chapman's frequency dependent model}

Neither Hudson's [Hudson, 1981] nor Thomsen's [Thomsen, 1995] theory account for frequency dependent behaviour. They can also not distinguish between the effects of many small cracks or fewer larger cracks, as they only consider the crack density (equation 7.10). A theoretical approach for frequency dependent crack anisotropy was developed by Chapman [2003], in order to retrieve information about fracture sizes, an important parameter in the exploration

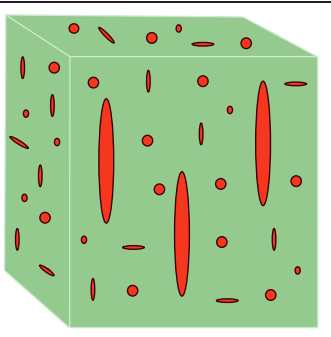

FIGURE 7.3 Network fracture model with pores, cracks and fractures industry.

The main advance in Chapman's model is the inclusion of three different scales of heterogeneities, i.e. cracks, pores and fractures building a network (figure 7.3). The existence of larger scale fractures also introduces velocity dispersion for lower seismic frequencies. The larger the fractures, the lower is the dispersion frequency band. Chapman's model incorporates fluid interaction at the mesoscale (fractures) and the microscale (pores and microcracks). These are crucial parameters for frequency dependent anisotropy.

The assumptions underlying Chapman's theory are:

- Grain-scale cracks and pores are distributed isotropically.

- Cracks are small compared to wavelengths.

- Fractures are larger than cracks and not interconnected, but they are connected to cracks and pores.

- The number of cracks and pores is much larger than the number of fractures.

The model describes the elastic stiffness of the porous cracked rock as:

$$
\begin{aligned}
C & =C_{0}-\phi_{p} C_{1}-\phi_{c} C_{2}-\phi_{f} C_{3} \\
& =C_{0}-\phi_{p} C_{1}-c_{1} \epsilon_{c} C_{2}-c_{2} \epsilon_{f} C_{3}
\end{aligned}
$$


where $\phi_{p, c, f}$ is the porosity of matrix, cracks or fractures, respectively, and $\epsilon_{c, f}$ is the density of cracks or fractures. $C_{0}$ is the isotropic elastic tensor of the matrix, $C_{1}, C_{2}$ and $C_{3}$ are the elastic stiffnesses of the pores, cracks and fractures inclusion materials, respectively, and $c_{1}$ and $c_{2}$ are constants. Relaxation time parameters $\tau_{m}$ and $\tau_{f}$, related to the squirt flow frequencies at the micro- and macroscale, link the processes between the different scales. The squirt flow frequency on the microscopic scale takes on values between the sonic and ultrasonic range. The flow in and out of the fractures is described by a larger time scale constant, depending on the size of the fracture:

$$
\tau_{f}=\frac{a_{f}}{a_{m}} \tau_{m},
$$

where $a_{f}$ is the fracture radius and $a_{m}$ is the grain radius.

Frequency dependence is introduced by a term $\left(1+i 2 \pi f \tau_{f}\right)^{-1}$ for the fluid flow in and out of the fractures and the term $\left(\frac{1+i 2 \pi f \gamma \tau_{m}}{1+i 2 \pi f \tau_{m}}\right)^{-1}$ for the pore scale flow, where $f$ is the frequency and $\gamma$ depends on the density and velocity in the matrix and fluid (cf. appendix F.3.2, equations F.46 - F.50 and F.51 - F.59).

Due to the introduction of a relatively low mesoscale squirt flow frequency and larger fractures, in contrast to earlier models (sections 7.2.1, 7.2.2, 7.2.3), dispersion can occur at seismic frequencies and therefore, frequency dependent anisotropy in the seismic band is predicted. The seismic frequency can no longer be regarded as the low frequency limit.

Chapman's expressions [Chapman, 2003] to calculate the components of the frequency dependent anisotropic elastic tensor can be expressed with the isotropic elastic tensor of the unfractured rock (e.g. the Lamé parameters $\mu$ and $\lambda$ ) and three correction terms, one for each porosity (fractures, cracks and pores). Those terms are functions of the frequency, but also depend on Lamé parameters, fluid properties, fracture length and time scale parameter (cf. table 7.2).

Because of the frequency dependence of the model, frequency independent reference moduli are needed in the calculations. Based on elastic parameters $\left(\lambda^{0}, \mu^{0}\right)$ derived from the velocities of the unfractured rock at a frequency $\omega_{0}$ and using the density of the saturated rock, these frequency independent reference moduli are defined as:

$$
\begin{aligned}
& \Lambda=\lambda^{0}+\phi_{p, c}\left(\lambda^{0}, \mu^{0}, \omega_{0}\right) \\
& \Upsilon=\mu^{0}+\phi_{p, c}\left(\lambda^{0}, \mu^{0}, \omega_{0}\right)
\end{aligned}
$$

Note, that the constant $\Lambda$ and $\Upsilon$ do not have any physical meaning. $\phi_{p, c}$ are the volume fractions of pores and cracks, respectively.

One difficult parameter is the micro-structural isotropic crack density $\epsilon_{e}$. It can be neglected if the frequency is lower than the micro-structural squirt flow and the matrix porosity is greater than the fracture porosity [Chapman, 2003]. Also, for small aspect ratios, the 
TABLE 7.2 Input parameters for Chapman model as used for the modelling in this study and in Chapman [2003] and Chapman et al. [2003].

\begin{tabular}{lll}
\hline Symbol & Meaning & Value \\
\hline$k$ & Permeability & $2 \cdot 10^{13} \mathrm{~Pa}$ \\
$\kappa_{f}$ & Fluid bulk modulus & $20 \mathrm{GPa}$ \\
$v_{P}$ & P-velocity & $4000 \mathrm{~m} / \mathrm{s}$ \\
$v_{S}$ & S-velocity & $2500 \mathrm{~m} / \mathrm{s}$ \\
$\rho$ & Density & $3.3 \mathrm{~g} / \mathrm{cm}^{3}$ \\
$\tau_{m}$ & Time constant for microstructure & $2 \cdot 10^{-5} \mathrm{~s}$ \\
$\phi_{p}$ & Volume fraction of pores (Porosity) & 0.1 \\
$\varepsilon_{c}$ & Crack density & 0.1 \\
$\varepsilon_{f}$ & Fracture density & 0.05 \\
$\zeta$ & Grain size & $200 \mu \mathrm{m}$ \\
$a_{f}$ & Fracture radius & $1 \mathrm{~m}$ \\
\hline
\end{tabular}

parameter $K_{c}$ can be neglected.

With these new reference moduli, the frequency dependent anisotropic elastic tensor can then be defined as:

$$
C_{i j k l}=C_{i j k l}^{i s o}-\varepsilon_{\mathcal{c}} C_{i j k l}^{1}\left(\lambda^{0}, \mu^{0}, \omega\right)-\phi_{p} C_{i j k l}^{2}\left(\lambda^{0}, \mu^{0}, \omega\right)-\varepsilon_{f} C_{i j k l}^{3}\left(\lambda^{0}, \mu^{0}, \omega\right)
$$

The exact expressions are given in appendix F.3. Table 7.2 describes the input parameters that are needed for the calculations.

Chapman et al. [2002] showed that the percentage anisotropy decreases with increasing frequency and that the frequency range over which this happens, depends on the fracture length. Pure shear is not affected by the frequency dependence, as it does not compress fractures and therefore induces no squirt flow. Substantial dispersion and attenuation can exist in the seismic frequency range. Velocities show a $\cos 2 \theta$ dependence on propagation direction relative to the crack normal at low frequencies and a $\cos 4 \theta$ dependence on propagation direction at high frequencies, in agreement with Thomsen [1995].

Still, even after reducing the independent parameters to a minimum, many parameters remain unknown and have to be adjusted; e.g. grain size, viscosity and the fluid parameters. The time scale parameter $\tau$ is probably the most crucial parameter, as it has a strong influence on the range of the frequency dependent anisotropy. Especially for calculations of deeper rocks, no samples are available, but laboratory experiments can help to assess some of these parameters [e.g. Takei, 2001, 2005]. 


\subsection{Frequency dependent fast polarisations}

Studies evaluating the frequency dependence of measured fast polarisations are rare. Liu et al. [2006] expanded Chapman's model [Chapman et al., 2002] to evaluate frequency dependent fast polarisations in the presence of two sets of cracks with different orientations and fracture densities. The presence of two crack sets with different orientation results in a less symmetric elastic tensor than a single crack set (table 7.1). The idea behind the calculations is that in presence of two crack sets with different scale lengths, lower frequency waves polarise according to the orientation of the larger scales, while higher frequency waves are more influenced by the smaller scales.

For normal incidence angle and vertical fractures, the shear-wave polarisation can be calculated from the crack orientations $\Phi_{1}$ and $\Phi_{2}$. With $\Phi=\frac{1}{2}\left(\Phi_{1}+\Phi_{2}\right)$ and $\Delta \Phi=\frac{1}{2}\left(\Phi_{1}-\Phi_{2}\right)$, the fast polarisation can be calculated as:

$$
\tan \left(2 \Phi_{\text {fast }}\right)=\frac{Z_{T}^{1} \sin (2(\Phi+\Delta \Phi))+Z_{T}^{2} G(\theta) \sin (2(\Phi-\Delta \Phi))}{Z_{T}^{1} \cos (2(\Phi+\Delta \Phi))+Z_{T}^{2} G(\theta) \cos (2(\Phi-\Delta \Phi))}
$$

where $Z_{T}$ are the fracture compliances, $\theta$ is the inclination of dipping cracks and $G(\theta)=$ $\cos ^{2}(2 \theta)-\sin ^{2}(\theta)$. Equation 7.31 can be simplified by replacing the fracture compliance $Z_{T}$ with the fracture density $e$, as they are linearly dependent:

$$
\tan \left(2 \Phi_{f a s t}\right)=\frac{e^{1} \sin (2(\Phi+\Delta \Phi))+e^{2} G(\theta) \sin (2(\Phi-\Delta \Phi))}{e^{1} \cos (2(\Phi+\Delta \Phi))+e^{2} G(\theta) \cos (2(\Phi-\Delta \Phi))}
$$

For non-vertical incidence angles or dipping cracks, the Christoffel equation has to be solved numerically and solutions become nontrivial.

The basic conclusions from the numerical modelling are:

- For normal incidence angle, shear-wave polarisations are independent of the properties of the background media.

- When two microcrack sets are combined, there is no frequency variation and the resulting polarisation is exactly between the two crack orientations, if the crack densities are equal. Otherwise they are approximately crack-density weighted averages.

- When one set of fractures is larger than the other, frequency dependence is observed for non-vertical incidence.

- For vertical incidence angles no frequency dependence is observed.

- With dipping fracture sets, the frequency dependence becomes more pronounced and is also apparent for (near) vertical incidence angles. 


\subsection{A combined model for the mantle}

In order to model anisotropy in the mantle, it is not clear which of the introduced models is the most suitable to use. Whereas Chapman's model [Chapman, 2003] is the most sophisticated, the existence of "open" cracks and pores is more applicable for the crust than for the mantle, where higher pressures force them to close. On the other hand, earlier models do not include the frequency dependent anisotropy, which has been observed for teleseismic shear-wave splitting

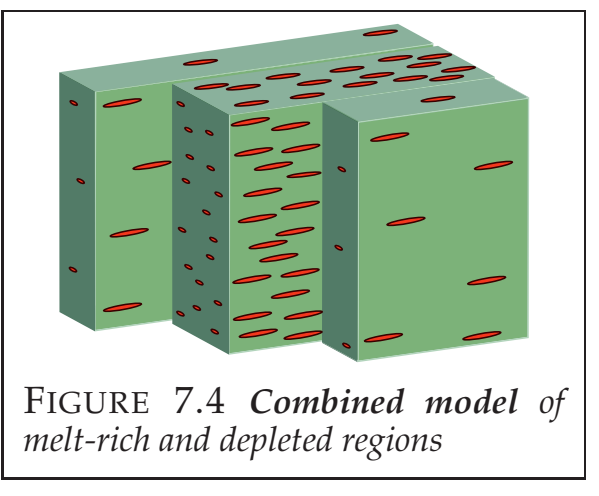
studies [Marson-Pidgeon \& Savage, 1997; Liu et al., 2001]. Considering the presence of melt can help to get around some of these problems.

Based on the theory of melt segregation caused by strain partitioning [Holtzman \& Kendall, 2008], a combined model approach can be applied to model this process in the mantle. The model comprises melt-rich and depleted layers with melt existing in oriented melt pockets (figure 7.4). The background (matrix) material can also be anisotropic. To model the elastic properties of such a medium, the effects of three mechanisms are combined:

1. Lattice-preferred orientation of the background matrix

2. Oriented melt pockets in two regions of the material; bands, i.e. areas where melt accumulates and melt depleted areas, using Hudson theory [Hudson, 1981]

3. Periodic thin layering of the depleted and melt-rich regions using Backus averaging [Backus, 1962]

In the model, the process of melt segregation for the medium is described by one single parameter, the degree of segregation $S_{t}$ [Holtzman et al., 2005]. All modelling parameters are coupled to $S_{t}$, so that the model only depends on this one parameter. A degree of segregation of $S_{t}=0$ stands for homogeneously distributed melt, whereas $S_{t}=1$ means that all the melt is segregated into bands. The volume fraction of bands $a_{b}$ and the melt fraction in the meltrich $\phi_{b}$ and melt-poor layer $\phi_{n}$ can be described by empirical formulas:

$$
\begin{aligned}
a_{b} & =S_{t} / \phi_{b}^{\prime} \\
\phi_{b}^{\prime} & =1+\left(\phi_{\max }-1\right)\left(S_{t}\right)^{c} \\
\phi_{n} & =\phi_{t}\left(1-S_{t}\right) /\left(1-a_{b}\right),
\end{aligned}
$$

where $\phi_{b}^{\prime}$ is the normalised melt fraction $\phi_{b}^{\prime}=\frac{\phi_{b}}{\phi_{t}}, \phi_{t}$ is the total melt fraction and $c$ is an empirical constant defined by Holtzman et al. [2005]. 
TABLE 7.3 Input parameter for oriented melt pocket modelling

\begin{tabular}{|c|c|c|}
\hline Theory & Matrix properties & Inclusion properties \\
\hline \hline Hudson & $\begin{array}{c}\text { North Island xenolith } \\
\text { (table A.1) }\end{array}$ & $\begin{array}{c}\lambda=14.91 \mathrm{GPa}, \mu=0 \mathrm{GPa}, \\
\rho=2.7 \frac{\mathrm{g}}{\mathrm{cm}^{3}}\end{array}$ \\
\hline Thomsen & $v_{P, d r y}=8 \frac{\mathrm{km}}{\mathrm{s}}, v_{S, d r y}=\frac{v_{P, d r y}}{1.7}$ & $v_{P, \text { saturated }}=8 \frac{\mathrm{km}}{\mathrm{s}}, v_{S, \text { saturated }}=\frac{o_{P, d r y}}{1.8}$ \\
\hline Tandon \& Weng & $v_{P}=8 \frac{\mathrm{km}}{\mathrm{s}}, v_{S}=\frac{v_{P}}{1.7}, \rho=2.8 \frac{\mathrm{g}}{\mathrm{cm}^{3}}$ & $\lambda=14.91 \mathrm{GPa}, \mu=1 \mathrm{GPa}$ \\
\hline
\end{tabular}

\subsection{Analytical modelling results}

\subsubsection{Anisotropy due to oriented melt}

In this section, the different frequency independent theories to calculate elastic properties due to preferred orientation of heterogeneities (sections 7.1 and 7.2) are applied to derive seismic anisotropy of mantle materials with melt inclusions. Input elastic properties to describe melt inclusions are based on Mainprice [1997]. The background medium properties are based on properties of the "North Island xenolith" [Duclos, 2005] and olivine (table A.1). The percentage anisotropy dependence on crack aspect ratio $\alpha$ and inclusion fraction is compared for the theories of Hudson [1981], Tandon \& Weng [1983] and Thomsen [1995]. Table 7.3 gives an overview of the parameters used in the modelling.

The three models agree in the general trend towards large anisotropic strength with high melt fractions (figure 7.5). Different input parameters are needed for the three theories, therefore it is hard to compare exact values modelled with the different theories. Tandon and Weng's theory [Tandon \& Weng, 1983] cannot model zero shear-wave velocity inclusions, therefore the shear modulus was set to a small value. Thomsen's theory [Thomsen, 1995] is the most complex one and requires a large range of parameters. Most of them are unknown for mantle rock and are therefore kept the same as in Thomsen's publication.

All models show a strong dependence on the volume fraction of the melt inclusion (figure 7.5). The dependence on aspect ratio is less pronounced for the Hudson and the Thomsen model. The maximum anisotropic strength gained with all the models, for a volume fraction of $10 \%$ and an aspect ratio of 0.1 , reaches about 10 to $20 \%$. The strong dependence on aspect ratio produces extremely high anisotropic strength for low aspect ratios in the Tandon and Weng model (figure 7.6(b)). Anisotropic strength reaches values of up to almost $90 \%$ for small aspect ratios and high volume fractions. This is here considered unrealistic and the model is probably only reliable for lower aspect ratios, in the region where anisotropic strength increases linearly with melt fraction (up to a volume fraction of about $2 \%$ ). The maximum anisotropic strength for the other two models stays in the range of 10 to $20 \%$ (figures $7.6(\mathrm{a})$ and $7.6(\mathrm{c})$ ). It has been stated that the Hudson model tends to give higher anisotropy than other models, but the deviations are found to be small if the cracks are fluid filled [Grechka, 2005].

Backus [1962] theory derives elastic properties for periodic layered media. In a coarse 


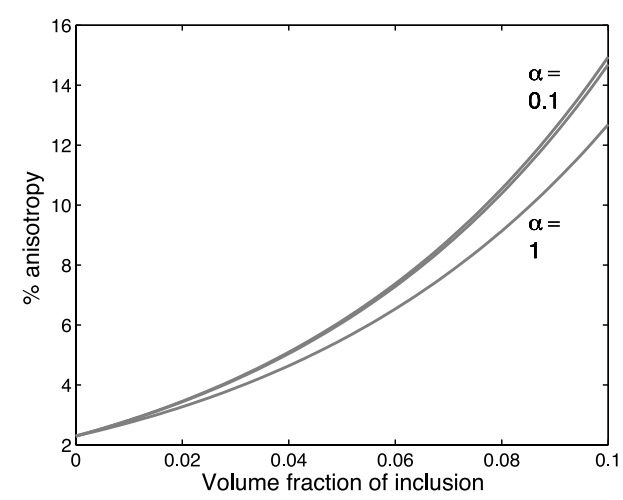

(a) Hudson [1981] theory

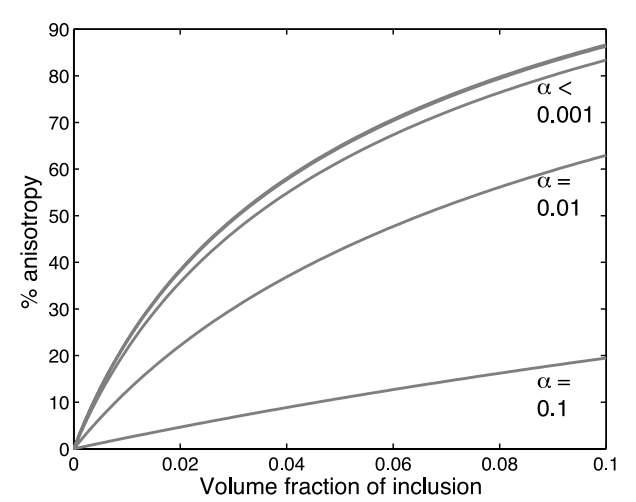

(b) Tandon \& Weng [1983] theory

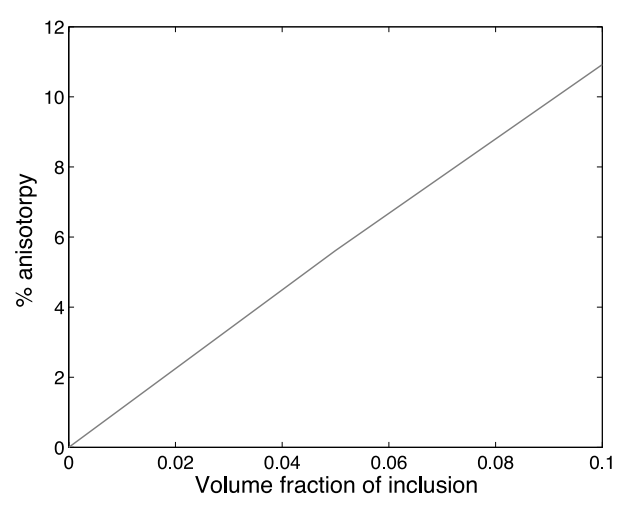

(c) Thomsen [1995] theory

FIGURE 7.5 Oriented melt pocket anisotropy with varying crack aspect ratio $\alpha$ compared for three different theories. Parameters used are given in table 7.3. Note that horizontal scales are the same but vertical axis scales vary between the plots.

approximation, following the ideas of Holtzman et al. [2003b], the occurrence of melt can be modelled in thin layers. A series of models, assuming thin low-velocity layers alternating with thicker layers of material with mantle properties, are modelled. The properties for the different layers are given in table 7.4. Figure 7.6 shows the resulting anisotropic strength. The strong velocity contrast of the melt layers to the background matrix creates high anisotropy even for low melt fractions. As little as $2 \%$ of melt can produce a maximum anisotropy of $30 \%$. This shows that layering is more effective in producing anisotropy than inclusions. The anisotropic strength increases with the volume fraction of melt and the velocity contrast. The model does not allow for low or a null shear-wave velocity, as they produce unrealistic values for the anisotropic strength of up to $200 \%$.

The combination of the layered and the inclusion model approaches (section 7.5) is used to model segregation of melt in bands [Holtzman \& Kendall, 2008]. Figure 7.7 shows variation of shear-wave velocities with incoming polarisation and the modelled anisotropic strength versus the segregation factor $S_{t}$ for the single layers and the combined medium. As before, 
TABLE 7.4 Periodic thin layer modelling parameters after Backus [1962]. Volume fraction c of matrix, maximum anisotropic strength $k$.

\begin{tabular}{|c|c|c|c|}
\hline \multicolumn{2}{|c|}{ Matrix Layer } & Melt Layer & \multirow{2}{*}{$\begin{array}{c}k \\
(\%)\end{array}$} \\
\hline \hline Properties & $c$ & Properties & $18 \%$ \\
Olivine (hexagonal) & 0.98 & $v_{p}=2500 \mathrm{~m} / \mathrm{s}, v_{s}=1300 \mathrm{~m} / \mathrm{s}$ & $18 \%$ \\
Olivine (hexagonal) & 0.98 & $\lambda=14.91 \mathrm{GPa}, \mu=1 \mathrm{GPa}$ & $30 \%$ \\
NIX (hexagonal) & 0.98 & $v_{p}=2500 \mathrm{~m} / \mathrm{s}, v_{s}=1300 \mathrm{~m} / \mathrm{s}$ & $16 \%$ \\
NIX (hexagonal) & 0.99 & $\lambda=14.91 \mathrm{GPa}, \mu=1 \mathrm{GPa}$ & $30 \%$ \\
Olivine (isotropic) & 0.98 & $v_{p}=2500 \mathrm{~m} / \mathrm{s}, v_{s}=1300 \mathrm{~m} / \mathrm{s}$ & $\sim 13 \%$ \\
Olivine (isotropic) & 0.99 & $\lambda=14.91 \mathrm{GPa}, \mu=1 \mathrm{GPa}$ & $\sim 30 \%$ \\
\hline
\end{tabular}
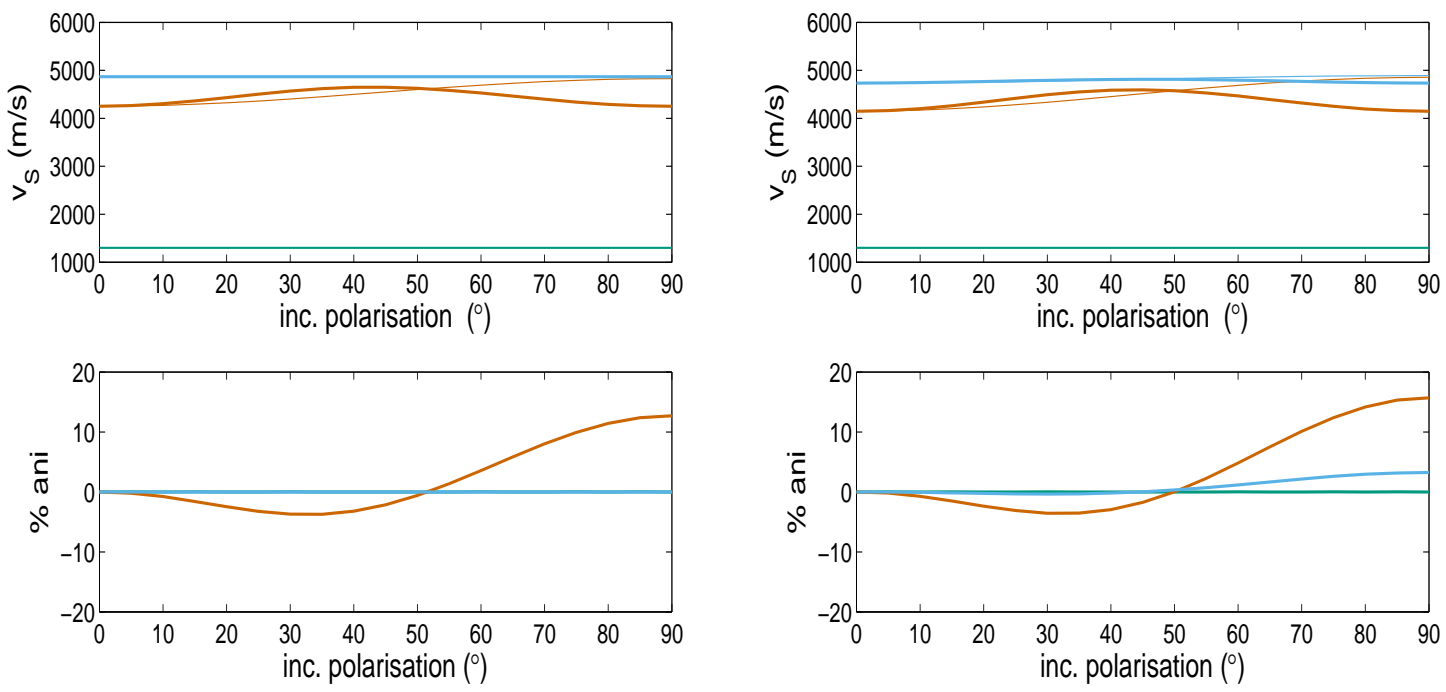

(a) Isotropic olivine $+2 \%$ melt

(b) North Island Xenolith $+2 \%$ melt

FIGURE 7.6 Periodic thin layering of melt. Anisotropy due to periodic layering of melt with mantle material calculated from Backus averaging. Top figures show shear-wave velocities (pure shear-wave - thin line, quasi shear-wave - thick line) variations and bottom figures anisotropic strength variations with incoming polarisation. Colours indicate variations in the melt layer (green), the matrix (blue) and the combined medium (orange).

the model input parameters are based on the North Island xenolith [Duclos, 2005] (table A.1) and melt described by Mainprice [1997]. Because the Backus averaging is defined for transversely isotropic media with a vertical symmetry axis, the elastic tensor of the North Island xenolith had to be modified for the modelling (table 7.5). Modelling the North Island xenolith background matrix and melt inclusions with null shear-wave velocity, anisotropic strength reaches maximum values of $6 \%$. This is lower than any of the single modelling approaches.

In contrast to Holtzman and Kendall's findings [Holtzman \& Kendall, 2008], the resulting anisotropic strength of the composite medium is not much higher than that of the initial matrix LPO (figure 7.7). In the melt-rich bands, however, the anisotropic strength reaches 
TABLE 7.5 Input parameters for combined model

\begin{tabular}{|c||c|}
\hline LPO & transversely isotropic North Island xenolith \\
& $C_{11}=217 \mathrm{GPa}, C_{33}=240 \mathrm{GPa}, C_{23}=73.5 \mathrm{GPa}, C_{44}=74 \mathrm{GPa}, C_{66}=79.94 \mathrm{GPa}$ \\
\hline OMP & $\rho=2.7 \mathrm{~g} / \mathrm{cm}^{3}, v_{P}=2.5 \mathrm{~km} / \mathrm{s}, v_{S}=0 \mathrm{~km} / \mathrm{s}$ \\
& total melt fraction: 0.03, max. volume fraction of bands: 0.25 \\
\hline
\end{tabular}
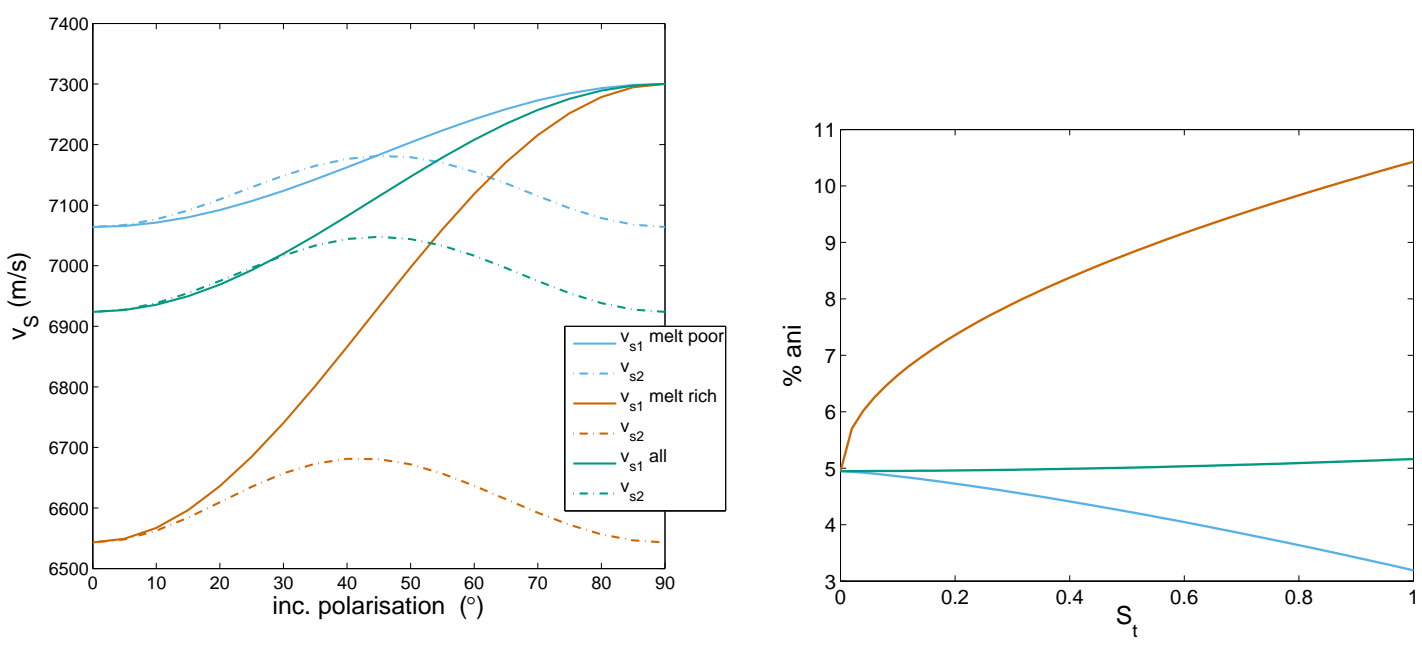

FIGURE 7.7 Anisotropy due to melt segregation-combined model. Left: Variation of shear-wave velocity with incoming polarisation for the single, melt-rich (orange) and melt-poor (blue) layers and the combined medium (green). Right: Variation in anisotropic strength with segregation factor for the single layers (orange, blue) and the combined medium (green).

high values. One reason for the discrepancy could be the Hudson averaging. An extended version of the averaging for full anisotropic tensors would be more appropriate to use, but the extension of the theory is beyond the scope of this work.

\subsubsection{Frequency dependent anisotropy due to oriented melt}

The models described above [Hudson, 1981; Tandon \& Weng, 1983; Thomsen, 1995] can explain high anisotropic strength, but they are limited to the upper and lower frequency limits and therefore are frequency independent. Chapman's theory [Chapman, 2003] is applied here to model variations in anisotropy with frequencies over the seismic frequency band. The calculations are based on the theory introduced in section 7.3.

Input parameters needed for the modelling are reduced to the most crucial parameters. Other parameters are set to constant values taken from the original publication, but can be changed easily in the code. A full list of all the input parameters is given in table 7.6. In order to model frequency dependent anisotropy due to melt-filled cracks, relaxation times according to melt squirt [Mavko \& Nur, 1975] need to be estimated. Melt squirt strongly depends on the velocity and therefore on the temperature. Other influencing parameters are the shape and connectivity of the melt inclusion and bulk modulus of melt and matrix. 
TABLE 7.6 Input parameters used to model melt inclusions in mantle. $\eta$ is the melt viscosity, the S-velocity in melt is set to zero. For description of other parameters see table 7.2.

\begin{tabular}{|c|c|c|c|c|c|c|c|c|c|}
\hline$v_{P}$ & $v_{S}$ & $\rho$ & $\varepsilon_{f}$ & $\varepsilon_{c}$ & $\phi_{p}$ & $\rho_{f}$ & $\tau_{m}$ & $\eta$ & $\kappa_{f}$ \\
\hline \hline $8 \mathrm{~km} / \mathrm{s}$ & $4.4 \mathrm{~km} / \mathrm{s}$ & $3.3 \mathrm{~g} / \mathrm{cm}^{3}$ & 0.05 & 0.05 & 0.05 & $3 \mathrm{~km} / \mathrm{s}$ & $1 \mathrm{~ms}$ & $100 \mathrm{~Pa} \cdot \mathrm{s}$ & $40 \mathrm{GPa}$ \\
\hline
\end{tabular}

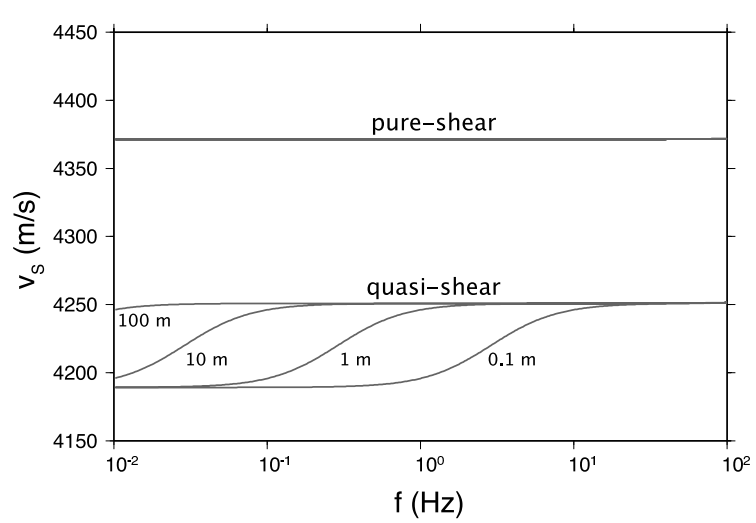

(a) Dispersion of quasi-shear and pure shear-waves

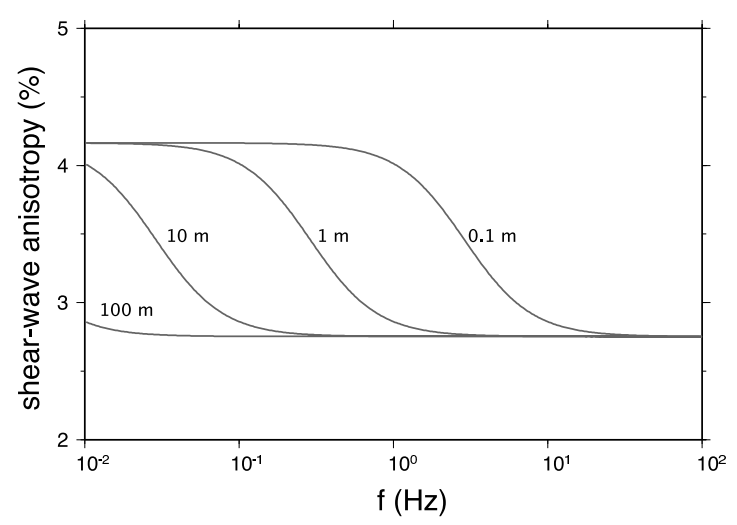

(b) Anisotropic strength

FIGURE 7.8 Frequency dependent anisotropy due to melt inclusions of various fracture sizes for the model parameters given in table 7.6.

It is invariant to the absolute scale [Hammond \& Humphreys, 2000], which is important in order to compare laboratory and field observations. Several relations to calculate melt squirt flow parameters exist [e.g. Mavko, 1980] in a general form of:

$$
\tau_{m}=\frac{A \eta_{m}}{\kappa \alpha^{B}}
$$

where $\eta_{m}$ is the melt viscosity, $\kappa$ the bulk modulus and $\alpha$ the aspect ratio of the melt inclusion. $A$ and $B$ are constants. Melt viscosities are assumed to be low (near $100 \mathrm{~Pa} \cdot \mathrm{s}$ ) [Hammond \& Humphreys, 2000] and relaxation times are generally proposed to be well below 1 s [Hammond \& Humphreys, 2000; Faul et al., 2004], i.e. above the sonic frequency band. Relaxation times have a direct influence on the fracture size needed to observe frequency dependent anisotropy in a certain frequency band.

The models show that melt-filled inclusions with length scales on the order of tens of metres are necessary to produce frequency dependent anisotropy in the teleseismic frequency range (SKS phases $\sim 0.1 \mathrm{~Hz}$; $c$. also spectra in appendix D.2). Higher frequencies are sensitive to smaller fracture sizes. The result strongly depends on the squirt flow. With lower squirt flow the frequency dependent changes occur for larger inclusions. The observed seismic anisotropy is high and on the same order as model results from Thomsen's and Hudson's model for similar crack densities around 3\%. 


\subsection{Discussion}

The various analytical models applied in this chapter all verify that the presence of melt can effectively produce anisotropy. Periodic thin layering of melt with other materials, such as olivine, is especially effective, reaching more than $15 \%$ anisotropy for only $2 \%$ melt (figure 7.6). Melt-filled inclusions also generate strong anisotropy, but values stay mostly lower than $10 \%$ for $2 \%$ melt inclusions (figure 7.5). The combined and the frequency dependent model approaches yield more moderate anisotropy, around 3-6\% (figures 7.7 and 7.8). This agrees with values found in similar studies [Holtzman \& Kendall, 2008; Tommasi et al., 2006].

The geometry of inclusions has a major effect on anisotropy. In the theory of Tandon \& Weng [1983], aspect ratios smaller than 0.001 yield unusually high anisotropy of more than $30 \%$ for $2 \%$ melt (figure $7.6(\mathrm{~b})$ ). It is therefore probably only reliable for aspect ratios higher than about 0.01. In a similar study, Ayele et al. [2004] model less than $1 \%$ melt inclusions with aspect ratios of 0.01 and get exceptionally high delay times of more than $4 \mathrm{~s}$ for $90 \mathrm{~km}$ path length, values that have never been observed in shear-wave splitting measurements. Aspect ratios between $10^{-1}$ to $10^{-2}$ are observed in peridotite samples with partial melt inclusions. Faul et al. [1994] measure disk-shape inclusions of aspect ratios $\alpha=0.05$ in olivine-basalt samples; Jousselin \& Mainprice [1998] determine an average aspect ratio of 0.3 in mantle peridotites of the Oman ophiolite.

The problem with the models is that the assessment of input parameters has to be based on a range of assumptions. However, modelling gives a good handle on ranges of anisotropic strength achievable from melt inclusions. All models yield values similar or higher than the average mantle anisotropy (4\%). This establishes the effectiveness of melt to produce the strong anisotropy as observed with teleseismic shear-wave splitting measurements in central North Island (chapter 5). The estimated $7-10 \%$ anisotropy needed in the CVR is not unrealistic in the presence of melt. Actually, 7-10\% anisotropy represent an intermediate value between the upper limit from the single mechanisms (PTL and SPO) and lower limits from the combined models. These results put a constraint on the properties that are realistic in the presence of melt and justify the properties included in the large-scale finite difference models discussed before (section 6.2)

Not enough is known about the actual state of melt in the mantle wedge. The fact that melt is generated at depth in the wedge and appears on the surface as active volcanism establishes the interconnectivity of melt and melt flow to the surface. Thus, the conditions are given for frequency dependent anisotropy due to squirt flow initiated by passing waves. Squirt flow is especially likely for low frequency waves, as the melt stays longer under the influence of the wave and has more time to move. The modelling shows that fractures of around $10 \mathrm{~m}$ scale generate frequency dependent anisotropy in the teleseismic frequency range. Such large-scale heterogeneities can be formed by melt segregation due to strain in the actively deforming subduction environment [Holtzman et al., 2003b] and are consistent 


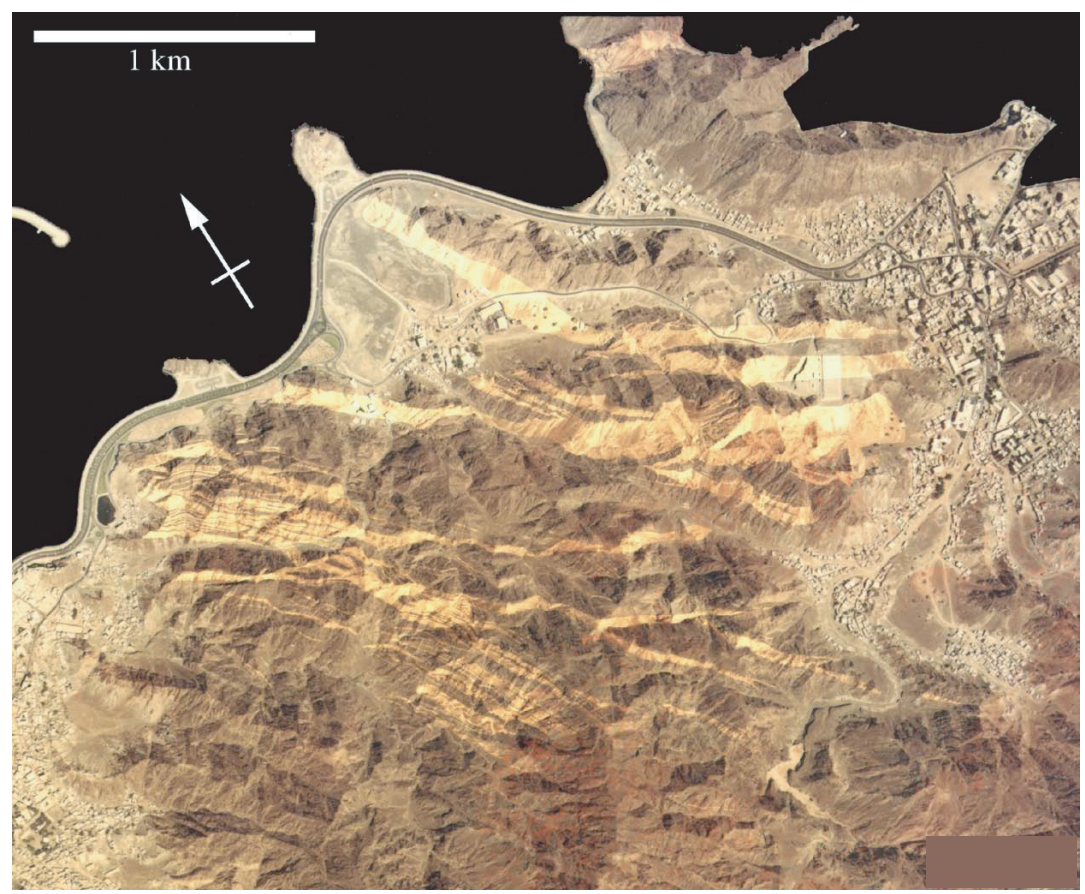

FIGURE 7.9 Example for large-scale melt bands in the Oman ophiolite [from Braun \& Kelemen, 2002].

with the expected spacings produced by this process under Earth conditions [Holtzman \& Kohlstedt, 2007]. Anisotropic properties of such large-scale features cannot be tested on rock samples in the laboratory. Field experiments need to be conducted in special settings, where large fractures have been found.

In the Kohistan Arc [Jagoutz et al., 2006] melt channels are about $500 \mathrm{~m}$ wide with spacing of about $10 \mathrm{~km}$. Other prominent examples for melt bands or networks of vertical dykes of this scale come from the Josephine peridotite, Oregon [Kelemen \& Dick, 1995], the Ingalls ophiolite [Kelemen et al., 2000], the Oman ophiolite [e.g. Kelemen et al., 2000; Braun \& Kelemen, 2002] (figure 7.9) and the Murray Ridge Massif, Canadian Codillera [Tommasi et al., 2006]. Tommasi et al. [2006] calculate S-wave anisotropy of up to about $10 \%$ for aspect ratios of 0.02 and melt bands containing $5 \%$ melt.

To produce trench-parallel fast polarisation in subduction zones, large-scale melt layers have to be aligned in steeply dipping planes and cracks need to align parallel to the trench. The CVR, with extension direction normal to the trench provides a suitable stress regime to form trench-parallel cracks and dyke intrusions in the crust [Rowland \& Sibson, 2001]. In the mantle wedge, vertical melt channels form if the melt rises faster than the plate moves away [Braun \& Sohn, 2003]. An additional role of such vertical melt-rich bands could be the lubrification of the mantle wedge corner flow.

In conclusion, the modelling of anisotropy due to melt in the wedge is still preliminary and relies on further results from interdisciplinary studies on the processes of melt generation, geometry and dynamics to improve model parameters. The uncertainty and the multitude of model parameters only allow for a conservative interpretation in consideration not 
to over-interpret the data. Nonetheless, it is certain that the presence of ordered melt, such as in aligned cracks of different shape or in bands, generates strong anisotropy. Probably both forms coexists in the mantle, as is suggested in the combined models. Therefore, it is important to consider structures over different scale lengths in anisotropic interpretations. Large-scale structures exceed the possibilities of laboratory measurements and this emphasises the need for field experiments covering several scale lengths. 


\section{Chapter 8}

\section{Evaluation of frequency dependent anisotropy}

With the discovery of frequency dependent anisotropy in laboratory measurements and the ability to model crack induced anisotropy and its frequency dependent effects, the need arises to look for such observations in field data. Active exploration data [e.g. Liu et al., 2003], as well as passive earthquake data [e.g. Liu et al., 2001; Marson-Pidgeon \& Savage, 1997; Tod \& Liu, 2002; Rümpker et al., 2003] exhibit evidence for frequency dependent anisotropy. Frequency dependence mainly prevails for delay times and is generally observed as an increase in delay times with decreasing frequencies. A variation is shown by Tod \& Liu [2002]. Their models predict an increase of delay times with frequency before it decreases. However, data evidence for this behaviour is poor. Frequency dependence of fast polarisations is less common in observations [Fouch \& Fischer, 1998] and models [Liu \& Bird, 2006].

In southern North Island, New Zealand, frequency dependent anisotropy is apparent as an increase in delay times with decreasing frequency for different phases [Marson-Pidgeon \& Savage, 1997]. For central North Island, initial examination of teleseismic shear-wave splitting data showed variations in frequency [Greve \& Savage, 2007]. Furthermore, distinct differences exist between shear-wave splitting parameters derived from local shearwaves [Morley et al., 2006; Audoine et al., 2000] and from teleseismic shear-waves in the CVR (cf. chapter 5 and Hofmann [2003]; Audoine et al. [2004]).

In this chapter, two different methods to evaluate frequency dependent anisotropy are introduced and applied to teleseismic shear-waves recorded in North Island. The first method applies band pass filtering with narrow, overlapping filters followed by shear-wave splitting measurements. The second method is a wavelet analysis. It is related to exploration industry methods that have been used successfully on active seismic data [Liu et al., 2003]. 


\subsection{Frequency dependent shear-wave splitting}

The most intuitive method to evaluate frequency dependent anisotropy is to apply the shearwave splitting method to waveforms filtered in different frequency bands. The use of overlapping filter bands ensures that no frequencies are skipped and the whole data range is covered. Because the relationship between frequency and wavelength is reciprocal (figure 8.1), constantly shifted frequency bands of constant widths have a much stronger effect on the wavelength than filters with constant period bands.

In order not to bias results, both constant frequency bands and constant period bands are used for filtering. Two-pole Butterworth-filtering with a frequency band of $0.2 \mathrm{~Hz}$ is applied for frequencies between 0.05 and $1 \mathrm{~Hz}$, with the lowpass frequency increasing in steps of $0.05 \mathrm{~Hz}$. In the second approach, Butterworth-filtering for constant period bands of $2 \mathrm{~s}$ is done for periods between 1 and $20 \mathrm{~s}$, with steps of $1 \mathrm{~s}$. It was shown on synthetic data that bandpass filtering itself does not introduce frequency dependence [Liu et al., 2003].

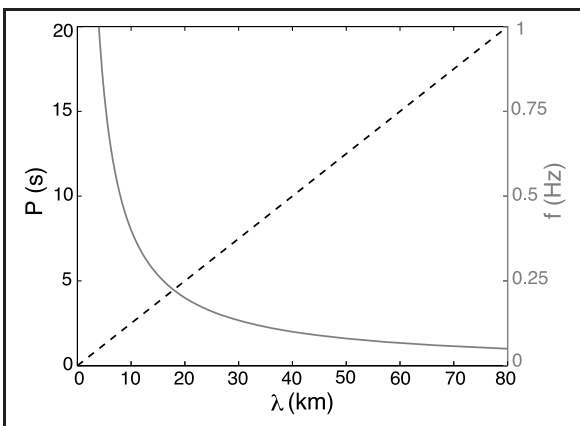

FIGURE 8.1 Period and frequency range covered by the analysis.

All the teleseismic SK(K)S-, S- and ScS-waveforms from the shear-wave splitting analysis that resulted in good and fair measurements (table E.1) are considered for the analysis. After filtering, evaluation of the shear-wave splitting parameters with the automated shear-wave splitting code follows the same procedure as described in section 5.3. All the frequency dependent measurements are evaluated manually and given marks ("good", "fair" or "poor") according to the quality of the measurement (section 5.3).

A drawback of narrow bandpass filtering is the generation of monofrequency waveforms. Shear-wave splitting measurements on such waveforms are prone to an effect called cycle skipping. Because of the similarity of the initial waveform to following waveforms, the trough of one waveform, e.g. the slow component, could be fitted to the peak or even the following trough of the other waveform, e.g. the fast component. In the presence of cycle skipping, delay times exhibit a time shift of integer multiples of half the dominant period of the waveform $\left(\delta t^{*}=\delta t \pm n \frac{T}{2}\right)$ and fast polarisation can show a $90^{\circ}$ ambiguity [Silver \& Savage, 1994].

The large amount of data produced by the frequency dependent analysis makes it necessary to apply different, more effective visualisation methods. Therefore, frequency dependent results are plotted in a colour-coded map of the variations of time delays or fast polarisations with frequency for all events. The event are sorted with the recording stations from northwest to southeast (figure 8.2). This representation accentuates systematic variations of splitting parameters with frequency as gradual colour variation. The centre value of the frequency or period range is used for plotting and "null" measurements are 


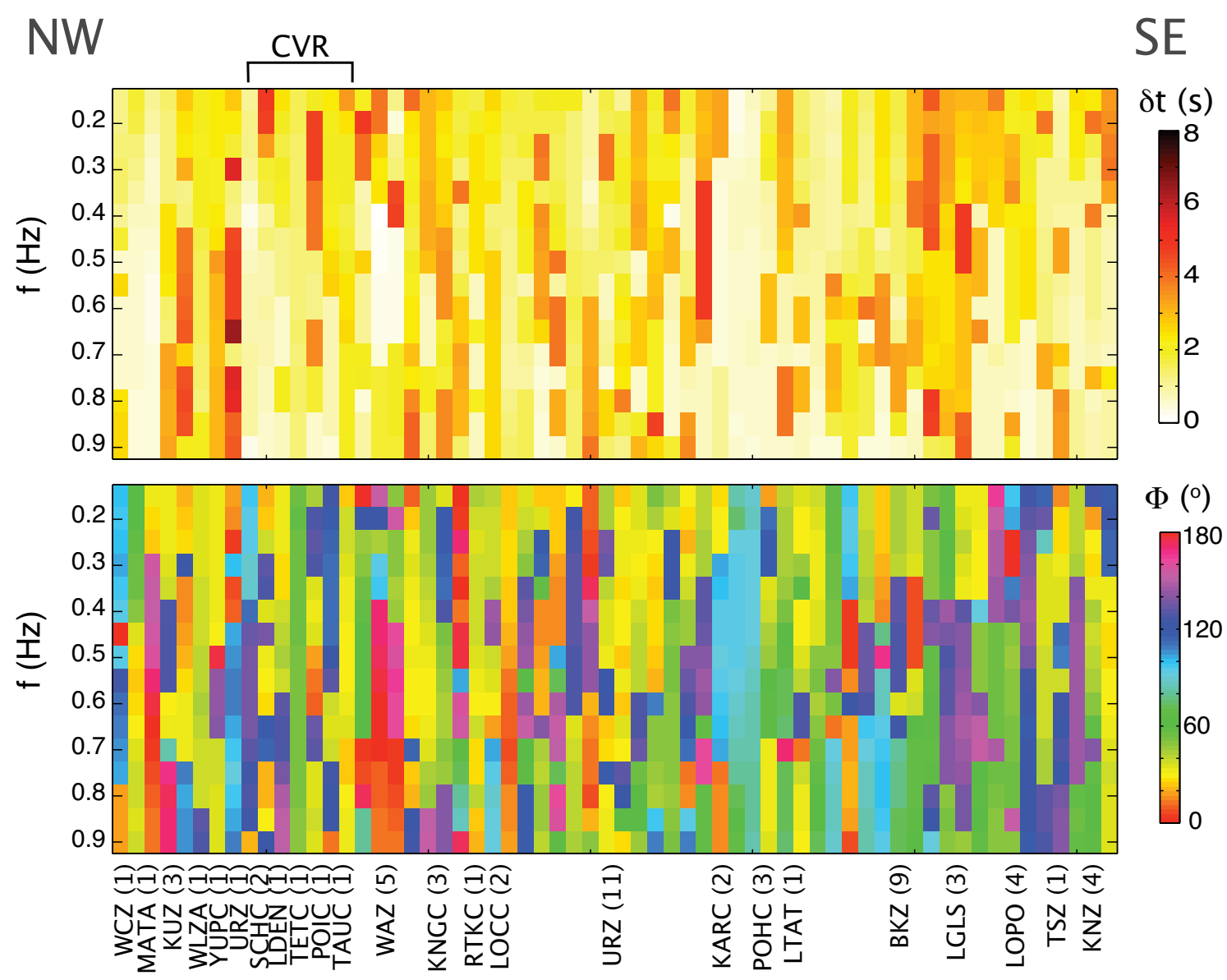

FIGURE 8.2 Results from frequency dependent shear-wave splitting. Splitting parameters for each event are plotted over the centre period used in filtering. Measurements are sorted according to recording station location from NW to SE. The number after the station name gives the number of events used at the site. Each column represents one event. Top: Delay time $\delta$ t. Bottom: Fast polarisation $\Phi$.

excluded.

A general trend toward higher delay times for longer periods could be inferred from the data. Fast polarisations are more scattered and no consistent change is apparent. However, overall splitting parameter seem to get more consistent with station KARC and east of it. West of KARC, some stations stay more consistent in delay times (e.g. POHC, LOCC), while others, sometimes neighbouring stations (e.g. LGLS, LTAT), show a gradual increase until a certain cut-off frequency. Because of the lack in consistent regional changes, the average behaviour is quantified by plotting the splitting parameters for all stations over frequency (figure 8.3). The trend for frequency dependent changes of delay time and fast polarisation for all single measurements is evaluated by fitting a linear trend line to the data. The linear trend is chosen as the most basic fit (first-order Taylor series approximation), as there is no indicator for any higher-order correlation. Different subsets of the dataset are used and compared (table 8.1). Figure 8.3 shows the trend for only the data rated "good". The centre periods for P-filtered data are converted to frequency for the evaluation of the trends to enable better comparison of the results from the two filtering methods. 


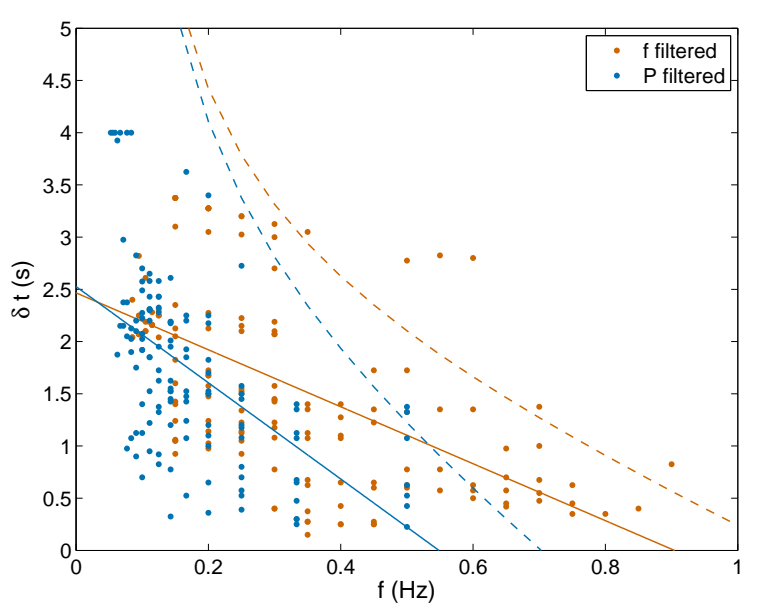

(a) Delay time

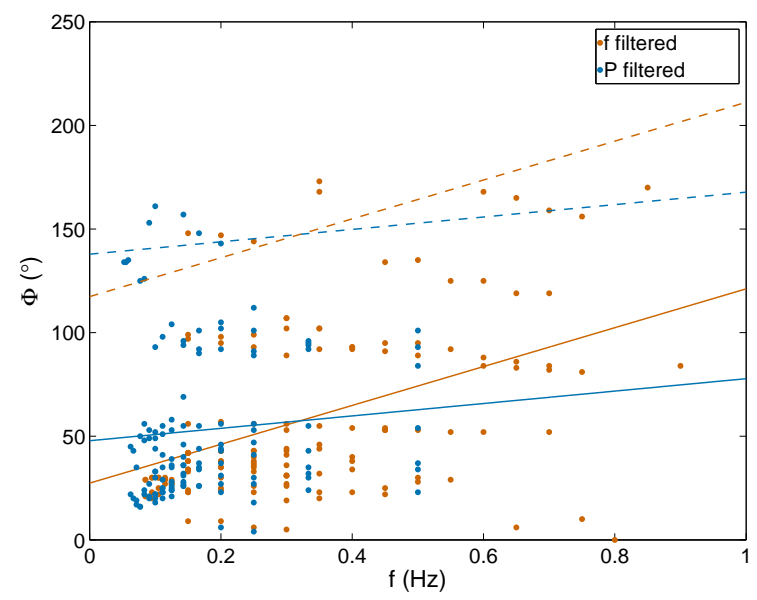

(b) Fast polarisation

FIGURE 8.3 Frequency dependent splitting parameters for all "good" data with best linear fit. Dashed line includes effects of cycle skipping. For details see text. Best-fit parameters are given in table 8.1.

TABLE 8.1 Best linear fit for frequency dependent shear-wave splitting dataset. Parameter with 95\% confidence bounds for linear fit: $y=A \cdot f_{f, P}+C$, with centre frequencies from filter band ranges designed based on frequency $f_{f}$ or on period $f_{P}$.

\begin{tabular}{|c|c|c|c|}
\hline Dataset & $\mathbf{y}$ & $\mathbf{A}$ & $\mathbf{C}$ \\
\hline \hline Good $\left(f_{P}\right)$ & $\delta t(\mathrm{~s})$ & $-5.82<-4.606<-3.392 \frac{\mathrm{s}}{\mathrm{Hz}}$ & $2.279<2.527<2.774 \mathrm{~s}$ \\
Good $\left(f_{f}\right)$ & $\delta t(\mathrm{~s})$ & $-3.378<-2.727<-2.076 \frac{\mathrm{s}}{\mathrm{Hz}}$ & $2.219<2.466<2.714 \mathrm{~s}$ \\
Good $\left(f_{P}\right)$ & $\Phi\left(^{\circ}\right)$ & $-29.91<29.94<89.79 \frac{\circ}{\mathrm{Hz}}$ & $35.66<47.84<60.03^{\circ}$ \\
Good $\left(f_{f}\right)$ & $\Phi\left(^{\circ}\right)$ & $62.29<93.77<125.3 \frac{\mathrm{Hz}}{\mathrm{Hz}}$ & $15.41<27.37<39.34^{\circ}$ \\
Good, marginal $\left(f_{P}\right)$ & $\delta t(\mathrm{~s})$ & $-4.792<-4.109<-3.425 \frac{\mathrm{s}}{\mathrm{Hz}}$ & $2.819<2.939<3.06 \mathrm{~s}$ \\
Good, marginal $\left(f_{f}\right)$ & $\delta t(\mathrm{~s})$ & $-2.469<-2.119<-1.77 \frac{\mathrm{s}}{\mathrm{Hz}}$ & $2.358<2.508<2.658 \mathrm{~s}$ \\
Good, marginal $\left(f_{P}\right)$ & $\Phi\left(^{\circ}\right)$ & $-24.24<5.396<35.03 \frac{\mathrm{H}}{\mathrm{Hz}}$ & $57.15<62.38<67.6^{\circ}$ \\
Good, marginal $\left(f_{f}\right)$ & $\Phi\left(^{\circ}\right)$ & $18.58<35.57<52.56 \frac{\mathrm{\circ}}{\mathrm{Hz}}$ & $45.9<53.19<60.48^{\circ}$ \\
All, no nulls $\left(f_{P}\right)$ & $\delta t(\mathrm{~s})$ & $-5.093<-4.562<-4.032 \frac{\mathrm{s}}{\mathrm{Hz}}$ & $3.444<3.54<3.636 \mathrm{~s}$ \\
All, no nulls $\left(f_{f}\right)$ & $\delta t(\mathrm{~s})$ & $-1.242<-0.9613<-0.6802 \frac{\mathrm{s}}{\mathrm{Hz}}$ & $2.31<2.467<2.624 \mathrm{~s}$ \\
All, no nulls $\left(f_{P}\right)$ & $\Phi\left(^{\circ}\right)$ & $-18.34<5.728<29.8 \frac{\circ}{\mathrm{Hz}}$ & $59.69<64.05<68.4^{\circ}$ \\
All, no nulls $\left(f_{f}\right)$ & $\Phi\left(^{\circ}\right)$ & $22.42<33.6<44.78 \frac{\mathrm{Hz}}{\mathrm{Hz}}$ & $44.64<50.88<57.12^{\circ}$ \\
Nulls $\left(f_{P}\right)$ & $\delta t(\mathrm{~s})$ & $-4.376<-3.821<-3.265 \frac{\mathrm{s}}{\mathrm{Hz}}$ & $3.189<3.291<3.392 \mathrm{~s}$ \\
Nulls $\left(f_{f}\right)$ & $\delta t(\mathrm{~s})$ & $-0.1447<0.1635<0.4718 \frac{\mathrm{s}}{\mathrm{Hz}}$ & $1.888<2.064<2.24 \mathrm{~s}$ \\
Nulls $\left(f_{P}\right)$ & $\Phi\left(^{\circ}\right)$ & $-18.87<5.176<29.22 \frac{\circ}{\mathrm{Hz}}$ & $74.1<78.5<82.91^{\circ}$ \\
Nulls $\left(f_{f}\right)$ & $\Phi\left(^{\circ}\right)$ & $-0.4914<0.04007<0.5715 \frac{\circ}{\mathrm{Hz}}$ & $72.63<78.83<85.02^{\circ}$ \\
\hline
\end{tabular}


The determined trends can only be considered reliable if the confidence areas are not too wide and do not include a change in signs. The inclusion of zero in the error bounds for $A$ means that the change is not significantly different from a constant behaviour. All, but the null data sub-sets, show significant changes in delay times on the order of $2-5 \mathrm{~s} / \mathrm{Hz}$. Note, that this is only valid for the frequency range used in the analysis $(0.05-1 \mathrm{~Hz})$. The inclusion of "poorer" data introduces a wider range for the frequency dependence. Trends in fast polarisations are not consistent within the data sets and mostly insignificant due to the inclusion of zero into the error bounds for $A$.

Both graphs (figures 8.3) seem to exhibit some kind of periodicity in the data points that could be caused by cycle skipping. Expected effects on splitting parameters due to cycle skipping are simulated by adding $T / 2$ to the best-fit delay time curve and $90^{\circ}$ to the best-fit fast polarisation curve (dashed lines in figure 8.3). For very low frequencies, effects of cycle skipping get large and create delay times reaching values exceeding $4 \mathrm{~s}$. An upper limit in the shear-wave splitting processing routine set to $4 \mathrm{~s}$ prevents frequency dependent splitting measurements of more than $4 \mathrm{~s}$ delay time. This limit was set lower than in the original shear-wave splitting analysis (chapter 5), as the highest measurements there yielded about $4 \mathrm{~s}$ and lower delay times are expected with the higher frequency filters. This helps to speed up processing time while retaining narrow enough windows to assure good measurements in the higher frequency range.

\subsection{Wavelet analysis}

Wavelet transforms [Morlet et al., 1982] are used similarly to Fourier transforms to extract frequency information from signals. To analyse time series by Fourier transform, a sliding segment of the length $T$ is needed. The advantage of the wavelet transform is its independence from any preimposed scaling interval such as $T$. Thus, it is more applicable for wider ranges of frequencies [Rioul \& Vetterli, 1991]. The continuous wavelet transform is defined as the convolution of the time sequence $x(t)$ with a wavelet $\psi$ :

$$
W(s, \tau)=\frac{1}{\sqrt{s}} \int_{-\infty}^{\infty} x(t) \psi^{*}\left(\frac{t-\tau}{s}\right) d t
$$

The $*$ indicates the complex conjugate. $s$ is a scale, corresponding to the frequency used in Fourier transforms. The usage of a single wavelet in the convolution makes the wavelet transform better designed to fit real data with finite limits, as opposed to the infinite sineand cosine-waves used in Fourier transforms.

The wavelet function must have zero mean and must be localised in time and frequency space [e.g. Torrence \& Compo, 1998]. An example for a wavelet commonly used in exploration seismology is the Morlet wavelet (figure 8.5(a)):

$$
\psi_{0}(\eta)=\pi^{-1 / 4} e^{i \omega_{0} \eta} e^{-\eta^{2} / 2}
$$




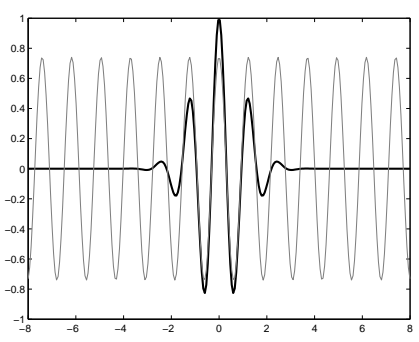

(a) Morlet wavelet

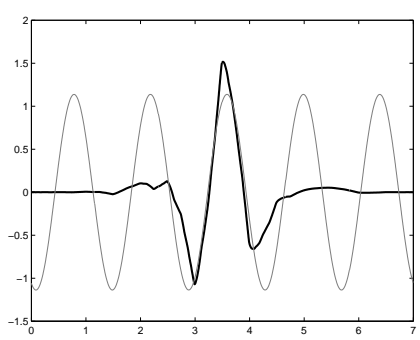

(b) Fourth order symlet

FIGURE 8.4 Wavelets used for continuous wavelet transform (black). The grey line indicates the centre frequency of the wavelet.

Its Fourier transform is a shifted Gaussian wavelet. Other wavelets often used for continuous wavelet transforms are nearly symmetrical wavelets, so-called "symlets" (figure 8.5(b)) [Daubechies, 1992, p. 194]. They are defined as regular filters with an orthonormal basis [Rioul \& Vetterli, 1991].

The wavelet transform results in an image of the amplitude variations of any features versus the scale $s$ and time $t$. This is the equivalent to a spectrogram from Fourier transforms analysis with the scale factor replacing the function of the frequency. It is therefore often called scalogram [Rioul \& Vetterli, 1991]. The results depend strongly on the wavelet that has been used. As the wavelet transform works with scales and does not directly relate to frequencies ( $c f$. equations 8.1), a relationship between scaling factor and frequency is needed. A way to obtain this is suggested in the Matlab ${ }^{\circledR}$ "wavelet toolbox". Based on the centre frequency $F_{c}$, a pseudo-frequency $F_{a}$ corresponding to the scale $s$ can be calculated as:

$$
F_{a}=\frac{F_{c}}{s \cdot \Delta}
$$

where $\Delta$ is the sampling period. The centre frequency of the wavelet $F_{c}$ describes the leading dominant (centre) frequency of the wavelet (figure 8.4). Thus, $F_{a}$ relates the wavelet to a purely periodic signal of the frequency $F_{c}$.

The wavelet transform can only give information about the frequency dependence of delay times and not fast polarisations. To perform the wavelet analysis on teleseismic shearwave data, the horizontal components need to be rotated into the fast and slow orientation first. Those orientations are taken from the previous shear-wave splitting analysis (table E.1) and kept constant for all frequencies. After rotation, the time frequency analysis is performed in Matlab ${ }^{\circledR}$ on both the fast and slow component. The analysis is performed with two types of wavelet, the Morlet wavelet and a fourth-order symlet ("sym4" in the Matlab ${ }^{\circledR}$ toolbox). Tests with other wavelet resulted in more cycle skipping (see also previous section 8.1), visible as period pattern in the resulting time-scale spectra (figure 8.5(c), (d)). A window of $50 \mathrm{~s}$ is used for the wavelet transform.

The scalograms for the fast and the slow shear-waves resulting from the continuous wave- 
a) Used wavelet sym4
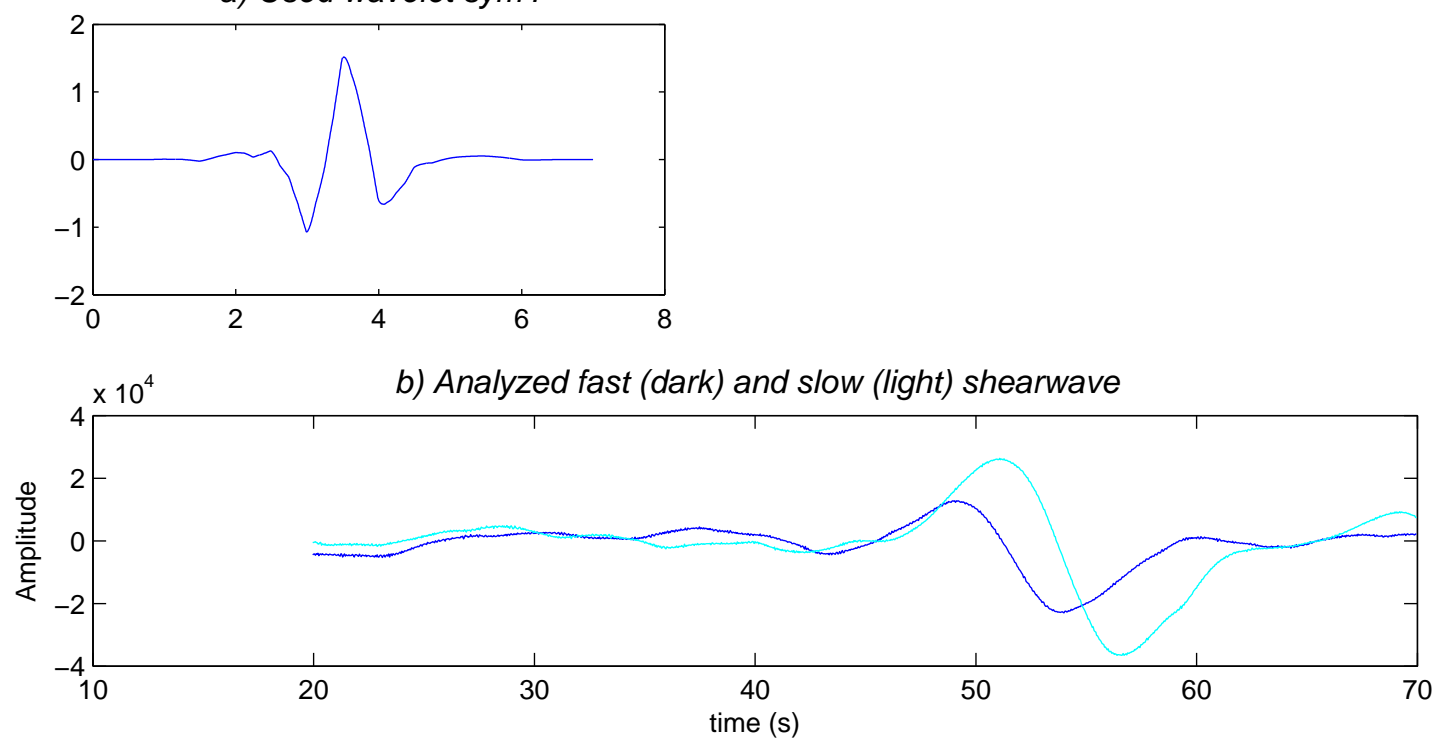

c) Continuous Transform, absolute coefficients, fast shearwave

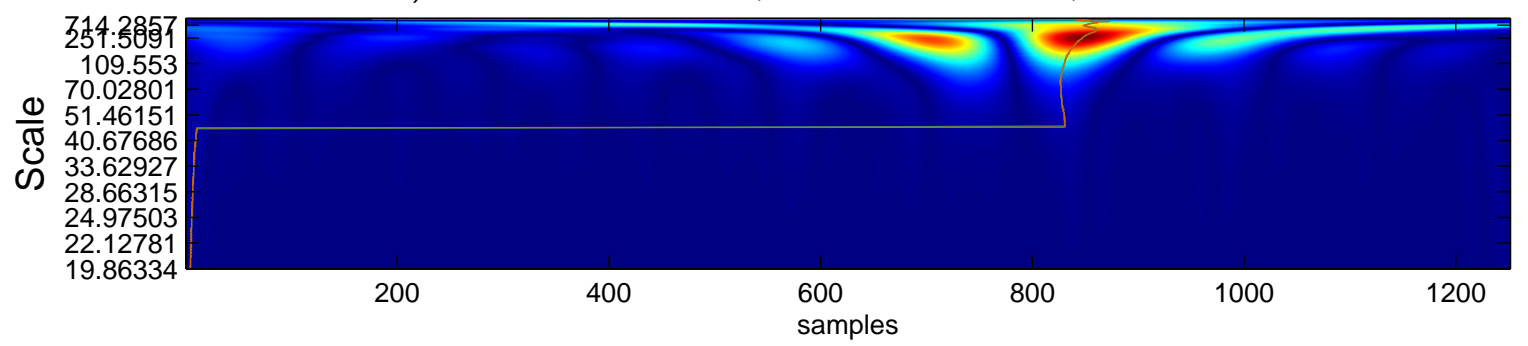

d) Continuous Transform, absolute coefficients, slow shearwave

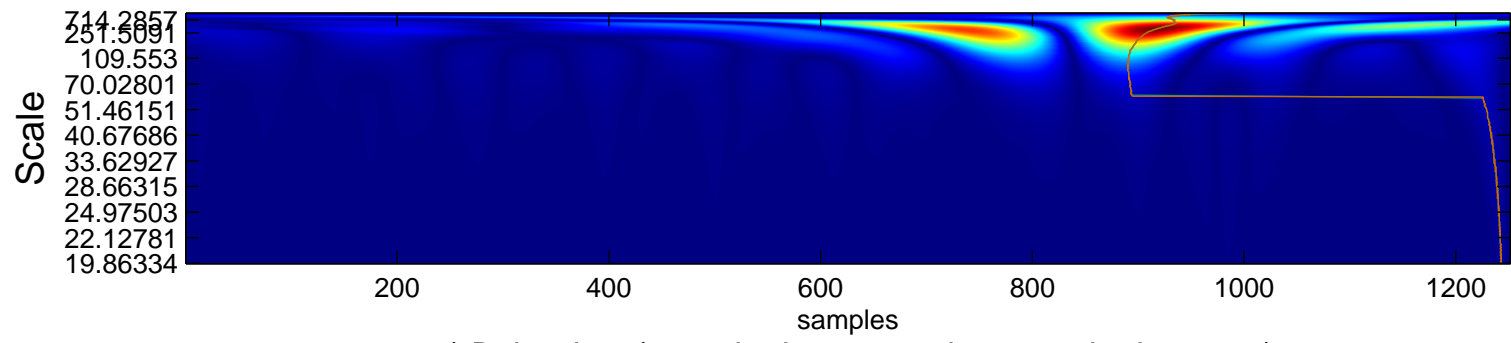

e) Delay time (smoothed, green and unsmoothed, orange)

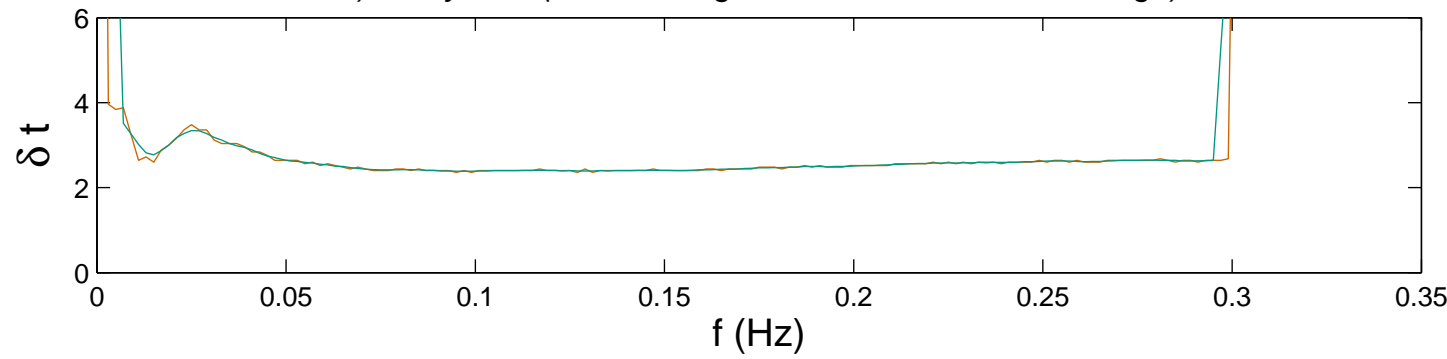

FIGURE 8.5 Estimating frequency dependent delay times with continuous wavelet transform. Example for wavelet transform with "sym4" symlet on fast and slow component for event 13/01/01, recorded at station KARC. For detailed description see text. 
let transform (see example figure 8.5(c), (d)) visualise the difference in travel times and the different frequency content between the two shear-waves. In the two spectra, the maximum amplitudes are picked together with the corresponding arrival times for each scaling factor. The maxima are marked by the red lines in the spectra in figures 8.5(c) and (d). The scales used correspond to frequencies between 0 and $1 \mathrm{~Hz}$. The picking is done automatically by filtering for the largest value per scaling factor. Outliers from the automatic picking are taken care of by a smoothed average. The difference between the picked travel times corresponding to the maximum amplitudes on the fast and slow components gives the delay times per scaling factor. Finally, the scaling factor are converted into their pseudo-frequency using equation 8.3 and plotted with the corresponding delay times (figure 8.5(e)).

The example in figure 8.5 reveals frequency dependent changes only for frequencies below $0.05 \mathrm{~Hz}$ (corresponding to scale factors higher than 100). Between 0.05 and about $0.3 \mathrm{~Hz}$, the delay time stays constant. For higher frequencies, no measurements are obtained.

All results from the continuous wavelet transform are presented as a summary map plot equivilant to figure 8.2. A continuous variation of delay time with frequency, visible as a gradual change of colour, can be observed for most of the data (figure 8.6). A trend towards lower delay times with increasing frequency is observable for the lower frequency range (0- $0.2 \mathrm{~Hz}$, figure 8.6). For higher frequencies (figure 8.6), however, the analysis does not provide sufficient results and data get sparse as delay times shift towards the upper and lower limits (cf. figure 8.5(c) and (d)).

\subsection{Discussion of frequency dependent analysis results}

Two methods were used to measure frequency dependent anisotropy on teleseismic shearwave data. In the first method, shear-wave splitting analysis is applied on data filtered with sliding, overlapping bandpass filters. The second method assesses frequency dependence in delay times using a continuous waveform analysis (CWT).

With both methods, it is hard to ascertain frequency dependent anisotropy. Some data show variations with frequency, but those variations cannot generally be ascribed to certain stations or regions. Delay times show more consistent behaviour than fast polarisations in that they tend to increase with longer wavelength (lower frequencies). Fast polarisations are more variable. Data was examined for regional variations in frequency dependent anisotropy, in analogy to the anisotropic regions determined by shear-wave splitting, but obvious pattern could not be identified.

There are two main problems that restrict the analysis. The major problem is the limited frequency content of the teleseismic shear-waves ( $c f$. spectra in section D.2). Generally, the frequency content of teleseismic shear-waves is not very broad. But in the case of the CVR, the frequency range becomes even more restricted through the attenuative properties of the volcanic ash infill of the backarc basin [e.g. Salmon et al., 2005] (cf. chapter 2.1). This could 


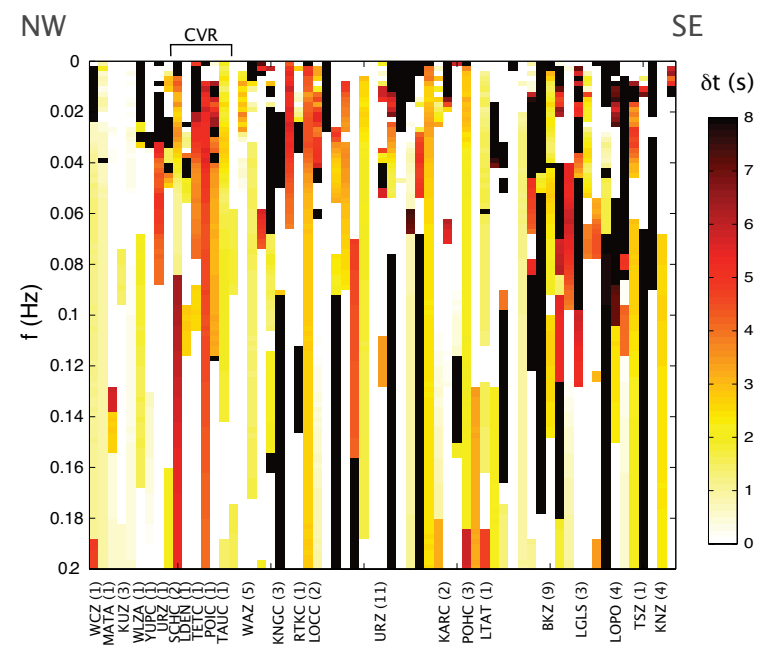

(a) CWT with symlet

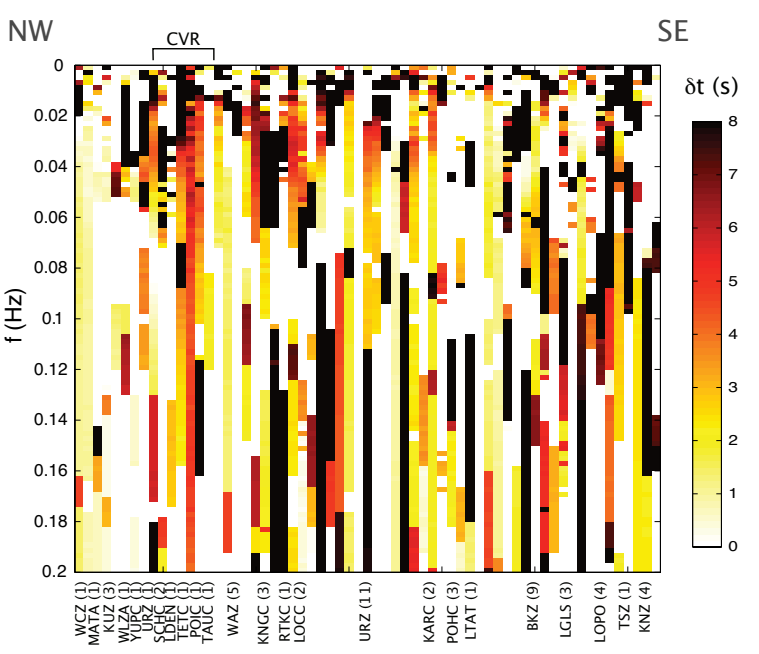

(b) CWT with Morlet wavelet

FIGURE 8.6 Frequency dependence in shear-wave splitting delay times from continuous wavelet transform. The frequency range covers only $0-0.2 \mathrm{~Hz}$, the band where most changes are observed (cf. text). Measurements are sorted according to recording station location from NW to SE. The number after the station name gives the number of events used at the site. The colour bar gives the colour-code for delay times. Two different wavelets (fourth-order symlet and Morlet-wavelet) are used.

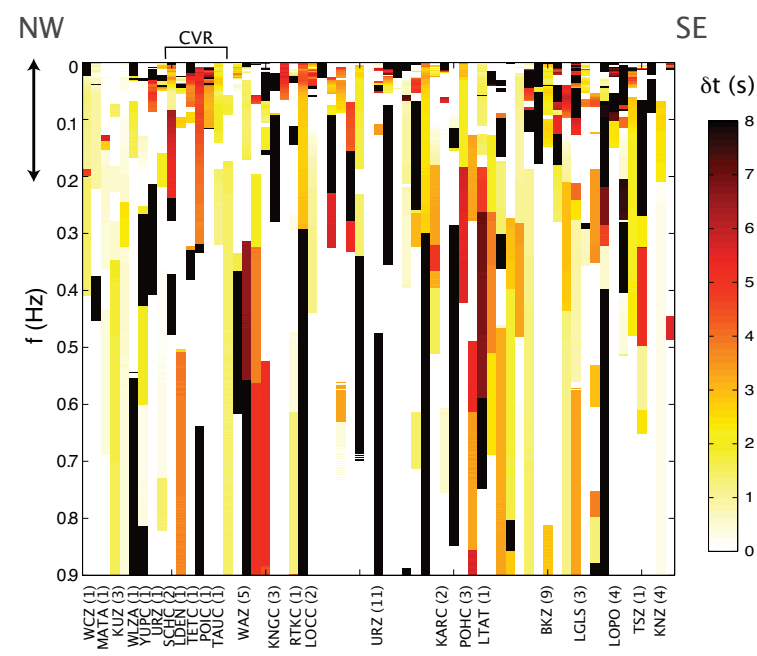

(c) CWT with symlet

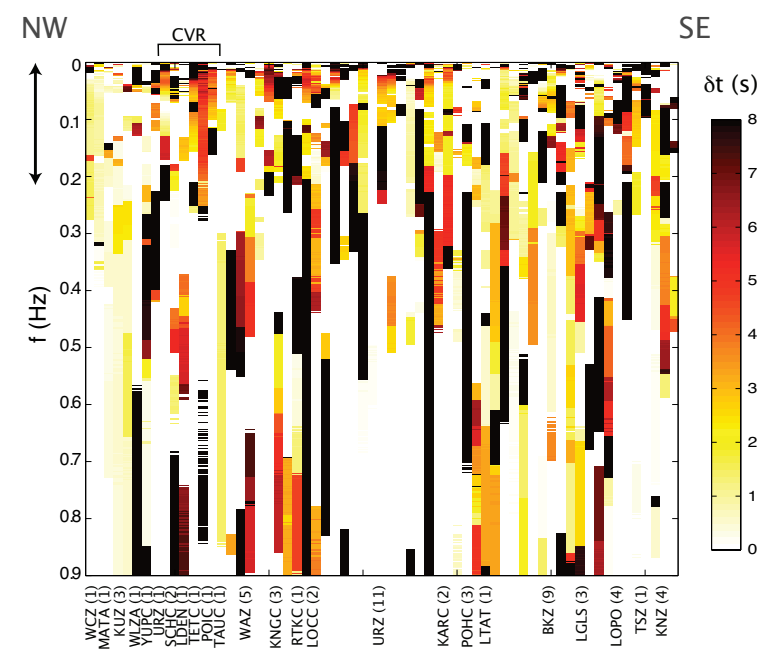

(d) CWT with Morlet wavelet

FIGURE 8.6 Frequency dependence in shear-wave splitting delay times from continuous wavelet transform. The frequency range covers $0-0.9 \mathrm{~Hz}$, which is the complete spectrum investigated. The colour bar gives the colour-code for delay times. Two different wavelets are used (Symlet and Morlet wavelet). The black arrow (left) indicated the most reliable frequency band (as shown in figure 8.6). 
be reflected in the low and near zero delay times measured for the higher frequencies from stations TAUC towards the west (figures 8.2 and 8.6) and would indicate that the attenuation is also evident with these long period waves. Another explanation for the low delay times in this region could be the influence of the apparent isotropy of western North Island. This influence would decrease towards the east with higher frequencies due to their smaller Fresnel zones. From station BKZ towards the east, information content increases in the data.

By looking at the results from the splitting analysis, it becomes obvious that frequency content is insufficient for analysis of frequencies above approximately $0.5 \mathrm{~Hz}$. The continuous wavelet transform analysis even covers a narrower frequency range up to about $0.2 \mathrm{~Hz}$. Results from frequency dependent shear-wave splitting analysis in this frequencies range are mainly rated "poor". However, the continuous wavelet transform additionally depends on the wavelets used, which might not be ideal for the analysis of teleseismic data. Improvements could be possible by using the original shear-wave as the convolution wavelet. Overall, a frequency bandwidth of less than one order of magnitude was feasible for the frequency dependent analysis. This is not sufficient to clearly test for frequency dependent anisotropy.

The second limiting factor lies in the performance of the measurements. Both methods can fail if periodicity affects the data. Narrow banded filtering enhances periodicity in the data and reduces the uniqueness of results. Therefore, the identification of "good" measurements becomes more difficult. Cycle skipping is a common problem for local shear-wave splitting measurements [Matcham et al., 2000; Teanby et al., 2004]. It results in non-uniqueness of splitting parameters; delay times are subject to shifts of integer multiples of half the dominant period of the waveform $\left(\delta t^{*}=\delta t \pm n \frac{T}{2}\right)$ and fast polarisations show a $90^{\circ}$-ambiguity. Frequency dependent shear-wave splitting can be affected by cycle skipping depending on the filters used. Additionally, the application of narrow banded overlapping filters multiplies the number of shear-wave splitting measurements and makes visual marking of the results more time-consuming. This demonstrates the need for more automated methods to evaluate the quality of an increasing amount of available data.

The attempt to plot results for single events in a colour-coded map in order to easier identify frequency dependent anisotropy exposed some regional variations (figures 8.2 and 8.6). But they are hard to quantify. Theoretically, due to the overlapping filtering technique used, gradual changes in the splitting parameters are expected if frequency dependent anisotropy is apparent. Gradual changes with frequency are also predicted by analytical models (section 7.3). If no frequency dependence exists, results are expected to stay constant over a certain frequency range, dependent on the predominant frequency of the measured phase. The overview of all results for individual events exhibits no general consistent changes. Some events show trends towards higher delay times for lower frequencies, while fast polarisation vary more "randomly". Nevertheless, high periods seem to have more consistency, yielding predominant fast polarisations of $\Phi \sim 40^{\circ}$. Events that gave "null" measurements 
in the initial shear-wave splitting analysis (chapter 5) are examined separately. Because the null splitting measurements were often gained from data filtered in a low frequency range (i.e. $0.04-0.1 \mathrm{~Hz}$ ), those events do still have the potential to show anisotropy in a different frequency band. However, results from "null" measurements are very variable and show no systematic changes, so they are not shown here.

The more general analysis plotting splitting parameter versus frequency/period makes it possible to apply a linear fit to the complete dataset (figure 8.3). It gives apparent frequency dependence in delay times of about $3 \pm 2 \mathrm{~s}$ per Hz. These results are consistent for all the subdata sets and the two ways of filtering. They are also on the order of changes implied for southern North Island [Marson-Pidgeon \& Savage, 1997]. The trend leads to very low delay times smaller than $0.5 \mathrm{~s}$ for frequencies approaching the range of local events around $1 \mathrm{~Hz}$. Even though this fits results from local shear-wave splitting measurements in central North Island [e.g. Morley et al., 2006], those values are also close to the resolution limit of the method applied here. Observed frequency dependent delay time changes for shallower structures in the USA yield only small changes in delay times on the order of $1 \%$ between 10 to $25 \mathrm{~Hz}$ [Liu et al., 2003; Maultzsch et al., 2003]. Thus, changes (gradients) in the higher frequency range are expected to be smaller than in the lower frequency range. This is also consistent with modelling results ( $c f$. section 7.6.2).

The linear fit for fast polarisation is poor and not consistent for the different subdata fits with wide error margins. Thus, no generalised trend can be inferred, which suggests that fast polarisations in central North Island do not depend on frequency. However, there are not many measurements in the higher frequency range, so that the differences in fast polarisation between local [Morley et al., 2006] and teleseismic shear-wave splitting (cf. chapter 5) cannot be tested for with the method applied here. Nevertheless, a gradual change in fast polarisation with frequency can be excluded. Frequency dependence in fast polarisations does not coincide with frequency dependence in delay times. Instead, it is expected in the presence of two sets of inclusions with different scale lengths (cf. section 7.4). The difference in the scale length is assumed to be on the order of the difference in frequencies, i.e. more than a magnitude.

In other studies, frequency dependent polarisation have been observed in field data [Fouch \& Fischer, 1998; Liu et al., 2003, 2007]. The frequency dependent fast polarisations observed by Fouch \& Fischer [1998] result from local events travelling through the wedge or near the slab-wedge interface. Shorter period filters yield broader variations in fast polarisations, while longer period filters give better constraints. This is interpreted as caused by the different resolution of the frequencies due to their Fresnel zones widths. In the presence of two different fracture sets of different scale-lengths, a clearer distinction for different frequencies is expected for non-vertical incidence ( $c f$. section 7.4).

The two principal mechanisms to cause frequency dependent anisotropy in delay times are scattering due to aligned heterogeneities and squirt flow in porous rocks. Scattering due to preferentially aligned heterogeneities produces frequency dependent anisotropy, if the 
scale of the heterogeneities is much smaller than the wavelength [Werner \& Shapiro, 1999]. Heterogeneities with scale estimates between 40 to $100 \mathrm{~m}$ are suggested to explain frequency dependent anisotropy observed in southern North Island [Marson-Pidgeon \& Savage, 1997]. The theory of frequency dependent anisotropy caused by fluid or squirt flow is discussed in section 7.3. It also shows that the frequency for which the changes in anisotropy are observed depend on the scale length of the large-scale fractures. To produce effects in the teleseismic frequency range, modelling results ( $c f$. section 7.3) yield fractures scales of tens of metres. Therefore, independent of the mechanism, heterogeneities on the same order of $10^{1}$ to $10^{2} \mathrm{~m}$ are necessary for frequency dependent effects in the frequency range considered in this study.

Ordered large-scale heterogeneities in a subduction zone setting can be formed by melt, existent in the mantle wedge. Initially homogeneously distributed melt can focus during buoyant ascent and combine to fast flowing channels [Aharonov et al., 1995]. These channels are expected to have spacings of 1-200 m [Spiegelman et al., 2001]. The main cause for the occurrence of these channels is the buoyancy of the melt. Melt is also considered to focus and form dyke-like channels due to stress [Daines \& Kohlstedt, 1997; Furukawa, 1993]. Another mechanism taking place under the influence of stress is the segregation of melt into bands [Holtzman et al., 2003b; Katz et al., 2006].

Such large-scale oriented melt inclusions are observed in obducted mantle samples in the field. The arguably most prominent example is the Oman ophiolite. It exhibits melt bands on the order of $10 \mathrm{~m}$ width [Braun \& Kelemen, 2002]. Another example in the Canadian Cordilleras is investigated by Tommasi et al. [2006]. The tabular dunites of several tens of centimetre thickness appear with spacings of $10^{1}$ to $10^{2} \mathrm{~m}$. The structure is interpreted to be obducted from about $70 \mathrm{~km}$ depth above an oblique subduction zone. The fast polarisation is assumed to have been trench-parallel and high anisotropy is calculate for the complex structure under the influence of melt.

In central North Island, basaltic dykes are found in the Tarawera rift [Nairn \& Cole, 1981]. Based on the location and composition of high-alumina basalts, Hiess et al. [2007] propose that melt under the TVZ rises in dyke swarms into the lower crust. This suggests that dykes are more likely to exist in shallower regions. Effective melt aspect ratios derived from logarithmic $v_{P} / v_{S}$-ratios (velocity reduction rate, $c f$. also section 9.4.2) in Japan indicate a disequilibrium texture and thus the focusing of melts into cracks, dikes or bands at depths of about 40-65 km [Takei, 2002; Nakajima et al., 2005]. This concept fits with the model of "Einats tree" [Aharonov et al., 1995]. At shallower depth, hydrofracturing of smaller cracks due to brittle failure can create melt path ways [Kelemen et al., 2000]. However, for depths below $40 \mathrm{~km}$, little is known about the actual mechanisms of melt transport. Tilmann et al. [2001] support the idea of channelled melt ascent from depth of $80 \mathrm{~km}$ to the crust based on moving velocities of melt.

In contrast, the frequency dependent anisotropy observed further south [Marson-Pidgeon \& Savage, 1997] cannot be caused by ordered melt channels, as no melt is apparent in this 
region (cf. section 2.1). However, a similar mechanism of ordered large-scale heterogeneities with different infill of less velocity contrast, probably in the slab, is possible and could also explain the overall smaller delay times in lower North Island. They can be formed by steeply dipping hydrated faults on top of the subducting plate, filled with hydrous mineral such as serpentine and talc [Faccenda et al., 2008].

Additional implication of the existence of melt in bands or isolated channels for the TVZ can be speculated. The wide, melt-lacking and therefore seismically fast regions in between the bands could provide a preferred path way for high frequency waves. This could explain the non-gradual differences between fast polarisations measured with local and teleseismic events. Concentrated melt provides effective transport to the surface [Kelemen et al., 1997], a possible cause for the high volcanic productivity of the TVZ. Additionally, the occurrence of melt could support the rifting process in the TVZ through heating and weakening of the lithosphere [Buck, 2004; Kendall et al., 2005].

\subsection{Conclusions}

Two methods to evaluate frequency dependent anisotropy are introduced and applied in a detailed analysis of teleseismic data recorded in central North Island. It difficult to ascertain frequency dependent changes for single events. Results from the wavelet analysis are not conclusive enough to either reject or prove the hypothesis of frequency dependent anisotropy in central North Island. The combined analysis of frequency dependent shearwave splitting parameters for all events yields no frequency dependence in fast polarisations. Delay times measured across the subduction zone in central North Island however, show a frequency dependent trend. This is consistent with the presence of a single preferred crack orientation and can provide a suitable explanation for the differences in delay times between local and teleseismic shear-wave splitting. The fact that fast polarisations for teleseismic and local shear-wave splitting measurements are perpendicular can be considered as frequency dependence, caused by the presence of two preferred crack orientation of different scale, but could not be tested for here.

Unfortunately, teleseismic events prove not to be ideal for a detailed, systematic frequency dependent analysis, as the frequency range covered is not broad enough to overlap with the range for local events. Furthermore, it is expected that the large Fresnel zone of the low frequency teleseismic waves smoothes frequency dependent effect over a broad area. This hinders the identification of regional frequency dependent effects, such as suggested for the CVR (cf. section 5.5).

The presence of melt in the mantle wedge is the most likely cause of the observed frequency dependence across central North Island. Its contribution can be twofold: due to squirt flow between interconnected fracture network or due to its accumulation in segregated bands or melt-rich dykes. Further implications of the presence of melt bands include 
an effective transport mechanism and effective heating resulting in high productivity volcanism and a hot and weak lithosphere with extensive rifting in the TVZ.

To gain more detailed information about frequency dependent anisotropy across the Hikurangi subduction zone, the approaches introduced here could be used with more extended data sets in the future. However, the insufficient frequency content of single phases will remain a major problem for the analysis. Comparison of various phases from different distances, such as local and teleseismic S- and regional ScS-phases extends the overall frequency range, but does not allow for direct comparison under the same conditions due to differences in travel paths, sources, etc. Yet, an identical study, applying the methods introduced here to local phases recorded on broadband stations, could provide valuable information in the intermediate frequency range, if events are big enough to produce longer period signal. 


\section{Chapter 9}

\section{Travel time residuals}

This chapter presents a pilot study carried out before the WCNIPSE line was established in the end of 2004. It is included to encourage and provide ideas for a future, more detailed study. In addition to introducing a method to evaluate travel time residuals, a preliminary analysis is presented based on teleseismic data recorded during the temporary CNIPSE deployment. The discussion outlines different possible interpretation approaches based on the preliminary data. The chapter finishes with suggestions to improve the study.

Travel time residuals are deviations of actual phase arrival times from expected arrival times at a station. They can be interpreted in terms of lateral variations in lithospheric structure and velocity. Additionally, backazimuthal variations in travel time residuals can provide information about anisotropy. One advantage in the use of travel time residuals lies in a broad backazimuthal coverage, as events from all epicentral distances can be used. Variations in incidence angles also ensure a large diversity in travel paths to sample different parts of the subsurface structure.

Several studies investigated travel time residuals for the New Zealand region before. In an early study of travel time residuals for regional earthquakes (arriving from a westerly backazimuth) recorded across North Island, Robinson [1976] found arrivals in western North Island to be late compared to those in eastern North Island by about $3 \mathrm{~s}$. He explains the difference as varying travel paths through the higher velocity descending Pacific lithosphere. But the model given is vague and the study only relies on events from the same backazimuth and source area. A study further to the north-east of New Zealand uses events from the Kermadec trench recorded on three islands to the east in the Pacific [Galea, 1993]. Large negative travel time residuals are observed (up to -16s), especially for shallow events. They are not restricted to the subducting lithosphere, but also observed in the lithosphere of the Pacific plate before it undergoes subduction. Characteristic azimuthal variations in the observed travel time residuals indicate anisotropy in the Pacific lithosphere. However, this cannot be verified for the region east of North Island. A more recent study investigates travel time residuals of S-wave for a model derived for best-fit P-wave arrivals [Harrison 


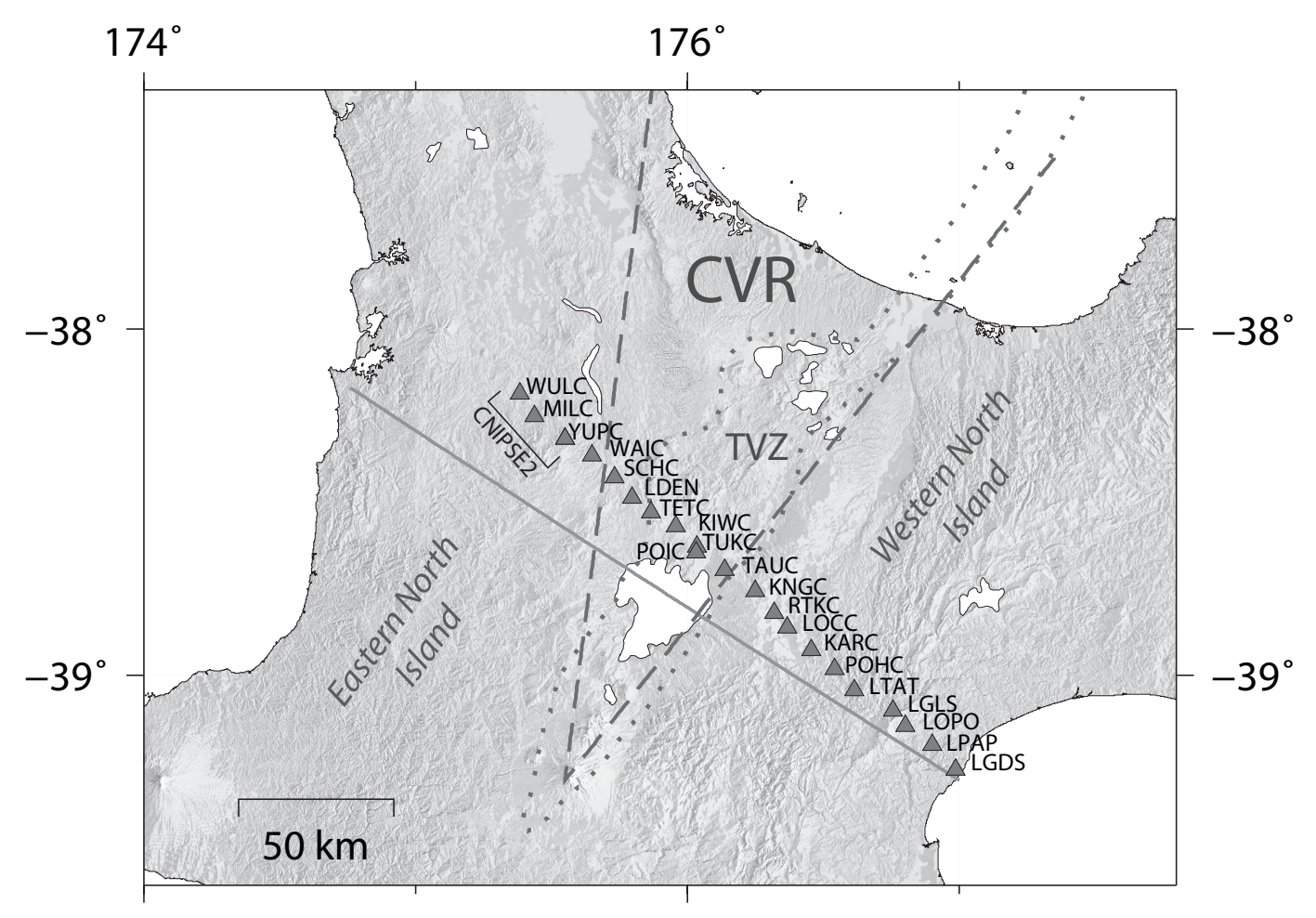

FIGURE 9.1 Station locations for travel time residual analysis. Triangles mark station locations; the solid grey line indicates the cross section studied by Harrison \& White [2006]. Boundaries of the Central Volcanic Region (CVR) and the young Taupo Volcanic Zone (TVZ) are shown as dashed lines.

\& White, 2006]. In contrast to the earlier studies, this study uses active source data and local events, sampling only the crust and parts of the upper mantle. Results show large Swave residuals (up to $2.4 \mathrm{~s}$ ) under the TVZ and west of it, if a Poisson ratio of 0.25 is used to convert the P-wave fitted model into an S-wave model. This indicates an anomalous S-wave structure under this region. East of the TVZ, the velocity structure appears more normal.

\subsection{Adaptive stacking method}

The evaluation of travel time residuals involves the comparison of actual travel times to predicted travel times. Travel time predictions are based on a velocity model. Determination of actual travel times can be based on different approaches, most of which include manual picking of the phase onset, often in combination with a cross-correlation between arrivals for station pairs [VanDecar \& Crosson, 1990]. Phase onsets can be hard to identify, especially if more phases than just the first $\mathrm{P}$-wave arrival are considered. The advantage of the adaptive stacking method [Rawlinson \& Kennett, 2004] used in this study is its independence of phase picks.

The adaptive stacking method performs a correlation between each recorded phase and a 

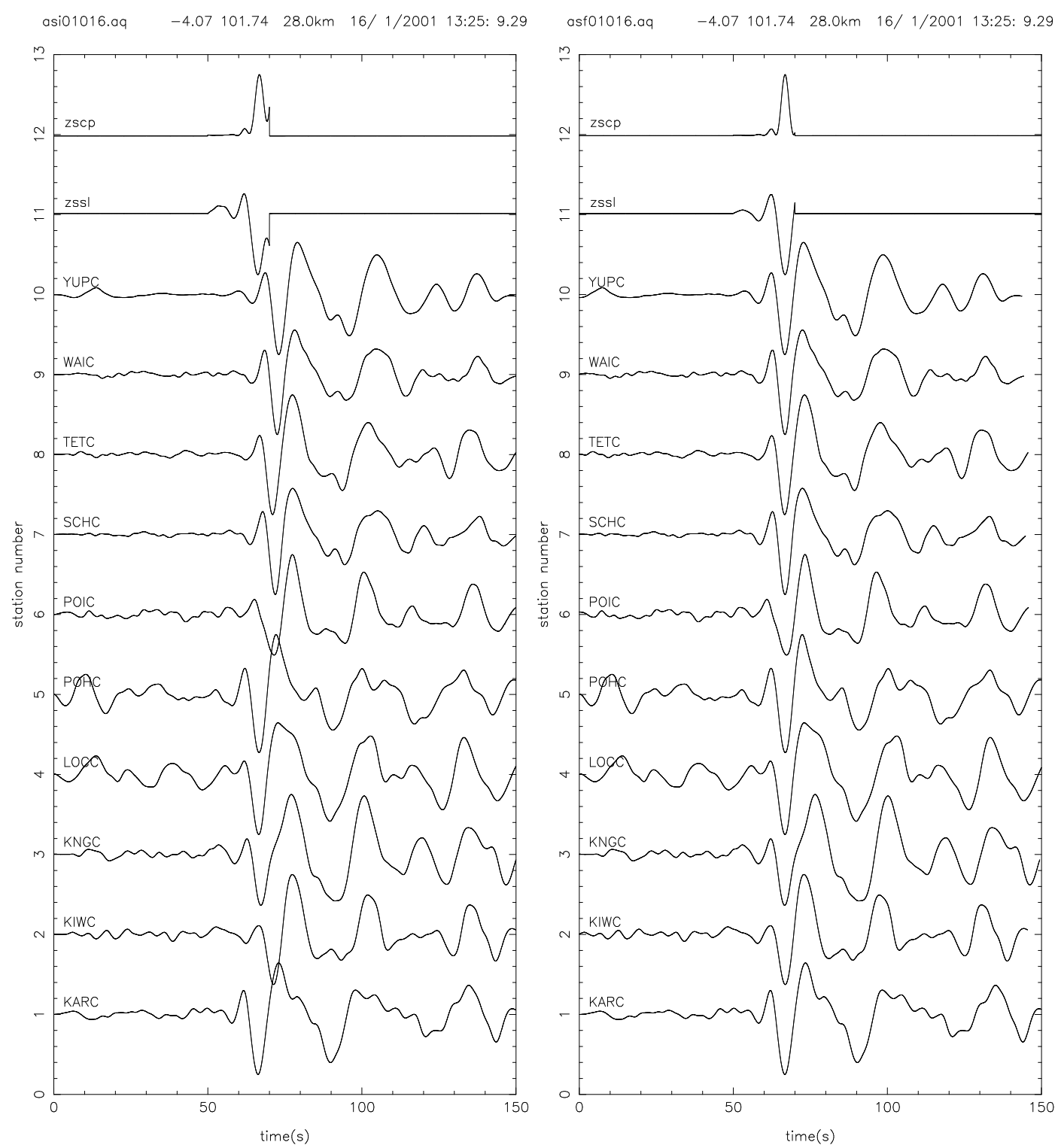

FIGURE 9.2 Example for adaptive stacking method on first S-phase of event 16/01/2001. Figures show the aligned traces for all the recording stations before (left) and after (right) adaptive stacking. Top two traces are the linear (trace 11, zssl) and squared (trace 12, zscp) stacked sum of all traces.

reference trace in an iterative process. To successfully apply the adaptive stacking method, the phases need to be roughly prealigned. The approximate alignment used here is based on the expected arrival time of the phase according to the ak135 global reference model [Kennett et al., 1995] (figure 9.2, left). Traces are cut $30 \mathrm{~s}$ before the expected phase arrival. Stacking of the prealigned trace creates a "typical waveform" used as reference trace for the correlation (figure 9.2, trace number 11 - zssl). The usage of a smoothed averaged reference trace is advantageous over other cross-correlation methods as it is more tolerant to variability in the waveforms. The additional calculation of the quadratic stack gives a handle on the spread in alignment between stations (figure 9.2, trace number 12 - zscp).

Each of the single traces is compared to the reference trace to improve alignment. An L3 
measure of misfit determines the best fit between reference trace and each single trace. The advantage of the $L 3$ over lower order misfit measures lies in an effective trace alignment and a more rapid convergence of the iterative process leading to very stable results [Rawlinson \& Kennett, 2004]. The aligning process is repeated iteratively, with the number of iterations set to 10. But convergence is often already achieved for fewer iterations. The adaptive stacking process results in direct estimates of travel time residuals from the ak135 model [Kennett et al., 1995].

In this study, two different data sets, CNIPSE and CNIPSE2, are evaluated for travel time residuals. Both lines have one station, YUPC, in common. Therefore, after adaptive stacking, the delay time determined for station YUPC is subtracted from all the other station delay times. The so obtained "relative" travel time residuals not only allow the simultaneous use of the two data sets, but they also reduce the effects of source and large-scale mantle heterogeneities, which would impose a constant offset to all station residuals.

\subsection{Data}

The data set considered for the travel time residual analysis consists of teleseismic events recorded along the CNIPSE line (figure 9.1). The main part of the line was deployed during the first half of 2001. The three westernmost stations extended the line for the second half of 2001. One site, station YUPC, stayed deployed for all of 2001 ( $c f$. chapter 4.1 and table B.1 for details on the deployment). All first P- and S-arrivals for events with magnitudes greater than 5.5, i.e. out of blocks 9 to 11 ( $c f$. table 4.1) are considered for the analysis. Only data with readily identifiable signal are used for the analysis. This results in 24 events for Pphases and 11 for S-phases (figure 9.3). Epicentral distances for these events vary between $20^{\circ}$ to $120^{\circ}$ and depths range from shallow to deep. Due to the short deployment time of the CNIPSE line, but also to the geographic location of New Zealand, backazimuthal coverage is limited and mainly restricted to the northwest.

The use of different broadband instrument types during the CNIPSE deployment makes it necessary to correct all datasets for instrument response before stacking (section 4.2.2). No other filtering is applied to the data. The size of the window can have important effects on the measured travel time residuals. In the presence of strong velocity anomalies and rapid structural changes, reflections, refractions and conversions originating close to the receiver cause distortions of the waveforms. The inclusion of strong later arrivals tends to introduce time shifts towards larger travel time residuals. To minimise the influence of secondary arrivals, smaller windows just around the onset of the direct phase are desired. However, in the adaptive stacking process, windows need to be large enough to include all the onsets across the record section (figure 9.2, section 9.1). Tests identified the best time window for effective alignment for P-phases as $5 \mathrm{~s}$ before and after the expected arrival and for S-phases as $5 \mathrm{~s}$ before and $15 \mathrm{~s}$ after the expected arrival. This window length typically includes about one period of the phase. 


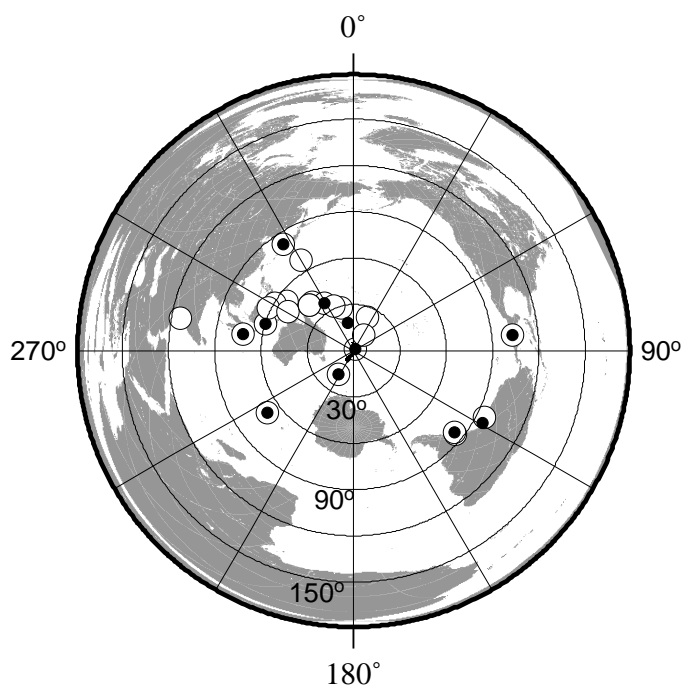

FIGURE 9.3 Events used for travel time analysis in equidistant azimuthal projection. White circles indicate travel time residuals calculated for P-phases, black circles for S-phases.

Travel time residuals for P-waves are calculated using recordings from the vertical component. For S-waves, recordings for both horizontal components, the north-south and the east-west component, are considered for the adaptive stacking process. All results from the adaptive stacking method were visually inspected to exclude obvious outliers and noisy traces from the results. In an additional test approach for S-waves, traces were rotated into approximate average fast and slow orientation, respectively. This approach did not produce any fundamentally different results (appendix H.1) and is therefore not further detailed here.

Generally, several corrections need to be applied to the raw travel time residuals. They include source corrections, usually corrected for by subtracting average travel time residuals for each event and station corrections, usually corrected for by subtracting average values for each station. Another elementary correction considers elevation. A rough maximum estimate for variations in travel time residuals due to elevation along the CNIPSE line can be given as $0.2 \mathrm{~s}$, assuming a maximum elevation change across the array of $1 \mathrm{~km}$ and an average P-wave velocity of $5 \mathrm{~km} / \mathrm{s}$.

Depending on the information already available for the study area, further correction e.g. due to crustal structure can be implemented. In the setting of a subduction zone, those corrections can get rather complex. Because of the preliminary character of this study, no corrections apart from subtraction of the travel time residuals for YUPC are applied.

\subsection{Travel time residuals along the CNIPSE line}

The adaptive stacking method applied on P-phases yields travel time residuals that vary between -3.84 and 4.48s, where negative residuals indicate earlier arrivals (figure 9.5(a)). Travel time residuals for S-phases recorded on north-south and east-west components vary from -10 to $7.26 \mathrm{~s}$, covering the same range for both components. Moreover, the character- 
istics of the travel time residual curves for both components are essentially the same. This is why only results for the north-south component are shown in figure 9.5(b). Results, especially for S-phases, strongly depend on backazimuth. Events arriving from northwest (backazimuths around $-60^{\circ}$ ) yield larger travel time residuals at the northwestern end of the line, decreasing and therefore indicating earlier arrivals towards southeast. Events arriving from southeast show slightly increasing travel time residuals from northwest to southeast for P-phases, but are more consistent in arrival times for S-phases. There are two events arriving from north (light blue circles in figure 9.4). For P-phases, they exhibit the strongest decrease in travel time residuals towards the southeast. S-phases show the opposite trend, with high travel time residuals increasing to the southeast. However, for S-phases this result is only based on one event.

A distinct change in the characteristic of travel time residuals takes place around stations TETC to TAUC, located in the Central Volcanic Region (CVR). Travel time residuals increase more steeply in the CVR, especially around station TETC. Outside the CVR, east of station TAUC, travel time residuals stay more consistent, with shallower gradients.

Plotting travel time residuals as a function of backazimuth and incidence angle gives information about the depth of the velocity anomaly. Most travel time residuals increase with shallower incidence angles (plotted further from the station, figure 9.5). But some exceptions, especially for S-waves, show the opposite trend.

\subsection{Discussion}

\subsubsection{Isotropic velocity and structural changes}

Travel time residuals are a useful tool to determine lateral variations in upper mantle velocities and lithospheric thickness, usually by inversion [e.g. Levin et al., 1995; James et al., 2001; Bastow et al., 2005]. Pure, uninverted travel time residuals are often evaluated in stable regions, such as shields and cratons [e.g. Bokelmann \& Silver, 2000; Engdahl \& Ritzwoller, 2001; Martynov et al., 2004; Fouch et al., 2004], where simple subsurface geometries dominate. In contrast to these studies, the use of travel time residuals in subduction zone settings [e.g. Plomerová et al., 2006] is less common and often reveals strong velocity contrasts [Engdahl \& Gubbins, 1987]. Here, the method is applied across the Hikurangi subduction zone, where subsurface structure is complex. The crust is known to be thinned under the CVR and mantle velocities are low [e.g. Harrison \& White, 2006; Stratford \& Stern, 2006]. Crustal thickness increases to the east, but is less constrained to the west of the CVR (cf. chapter 2.1). This complicates corrections for crustal structure (usually applied for the upper tens of kilometres).

The strongest changes in travel time residuals occur in the CVR, where crustal thinning is apparent. Evidence for shallow causes of travel time residuals under the CVR comes 


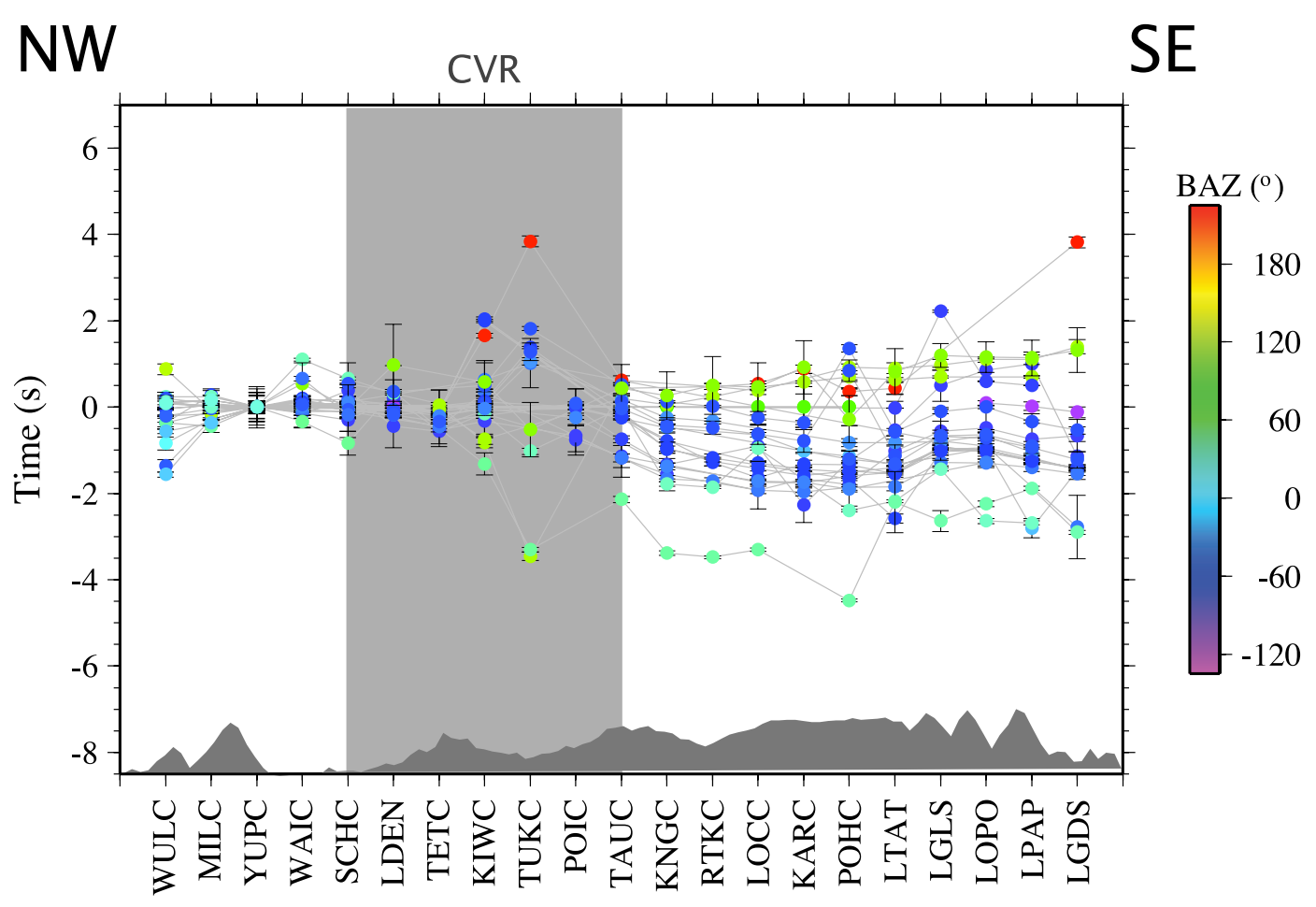

(a) P-phases

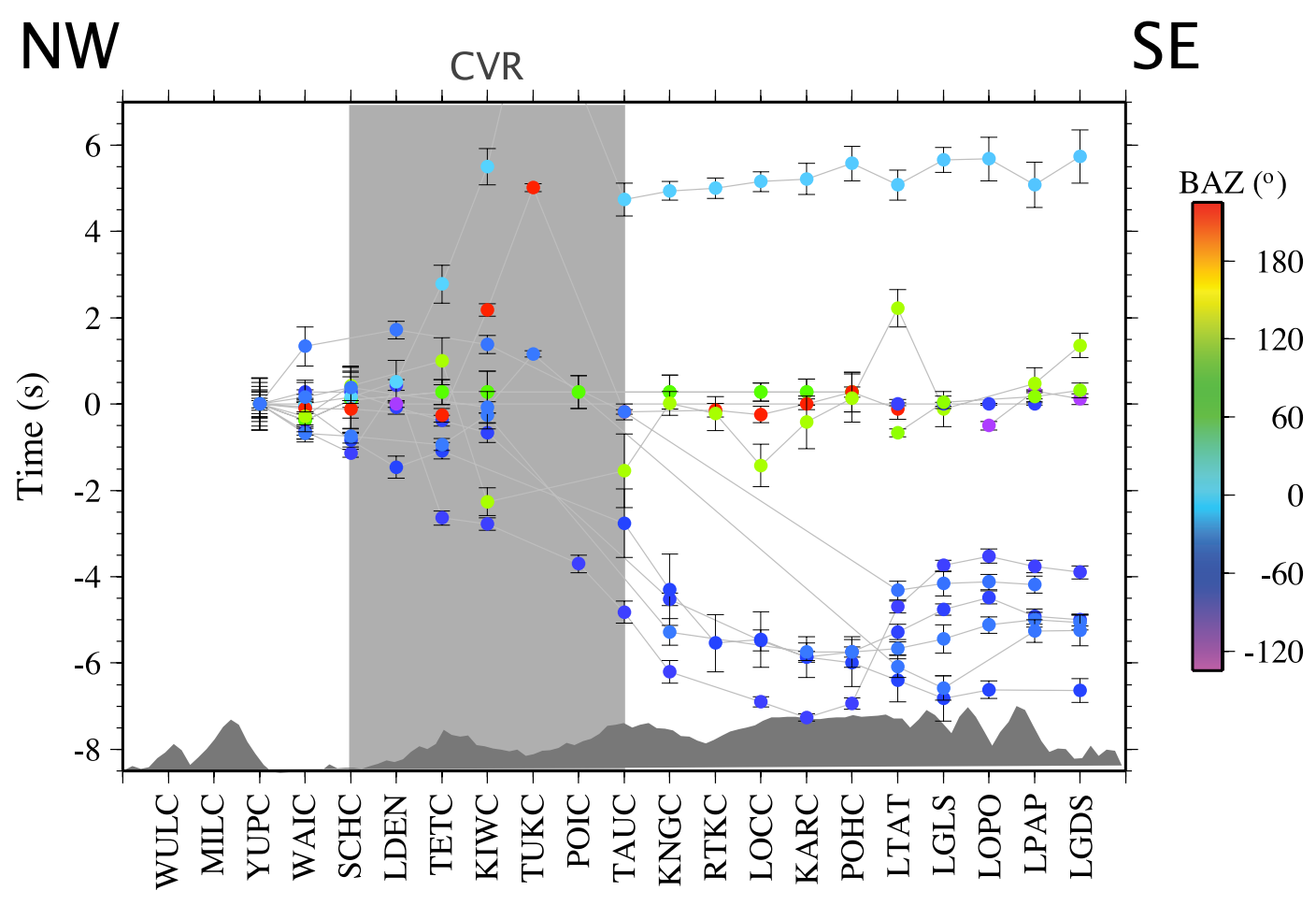

(b) S-phases (north-south component)

FIGURE 9.4 Travel time residuals for P-and S-waves. Colours of the data points represent the different backazimuths of the events (scale). Negative residuals indicate earlier arrivals relative to the reference station YUPC. Light grey areas mark stations located in the Central Volcanic Region (CVR). Dark grey illustrates topographical changes along the array, with maximum heights of about $1100 \mathrm{~m}$. One measurement for TUKC is off-scale. 


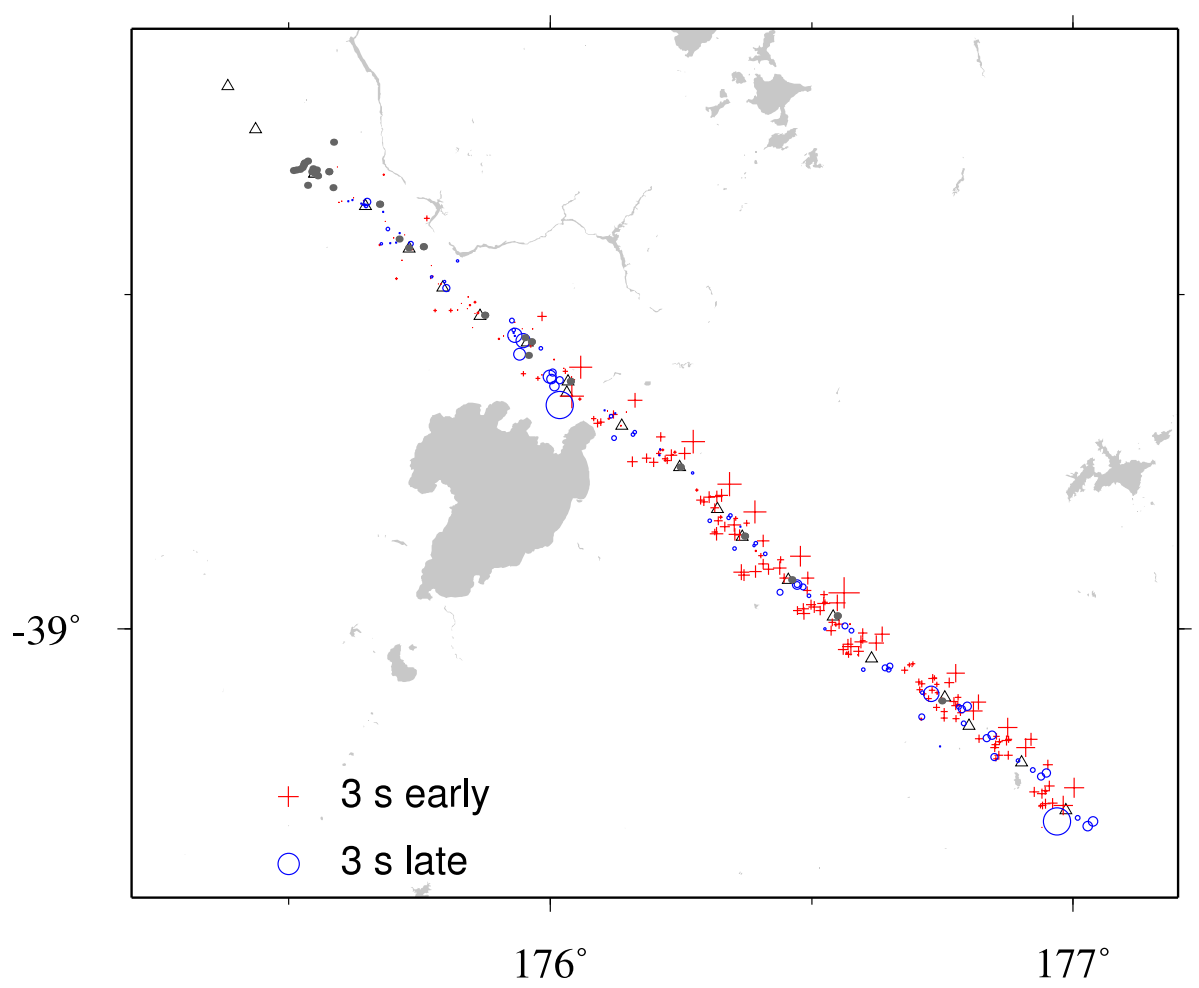

(a) P-phases

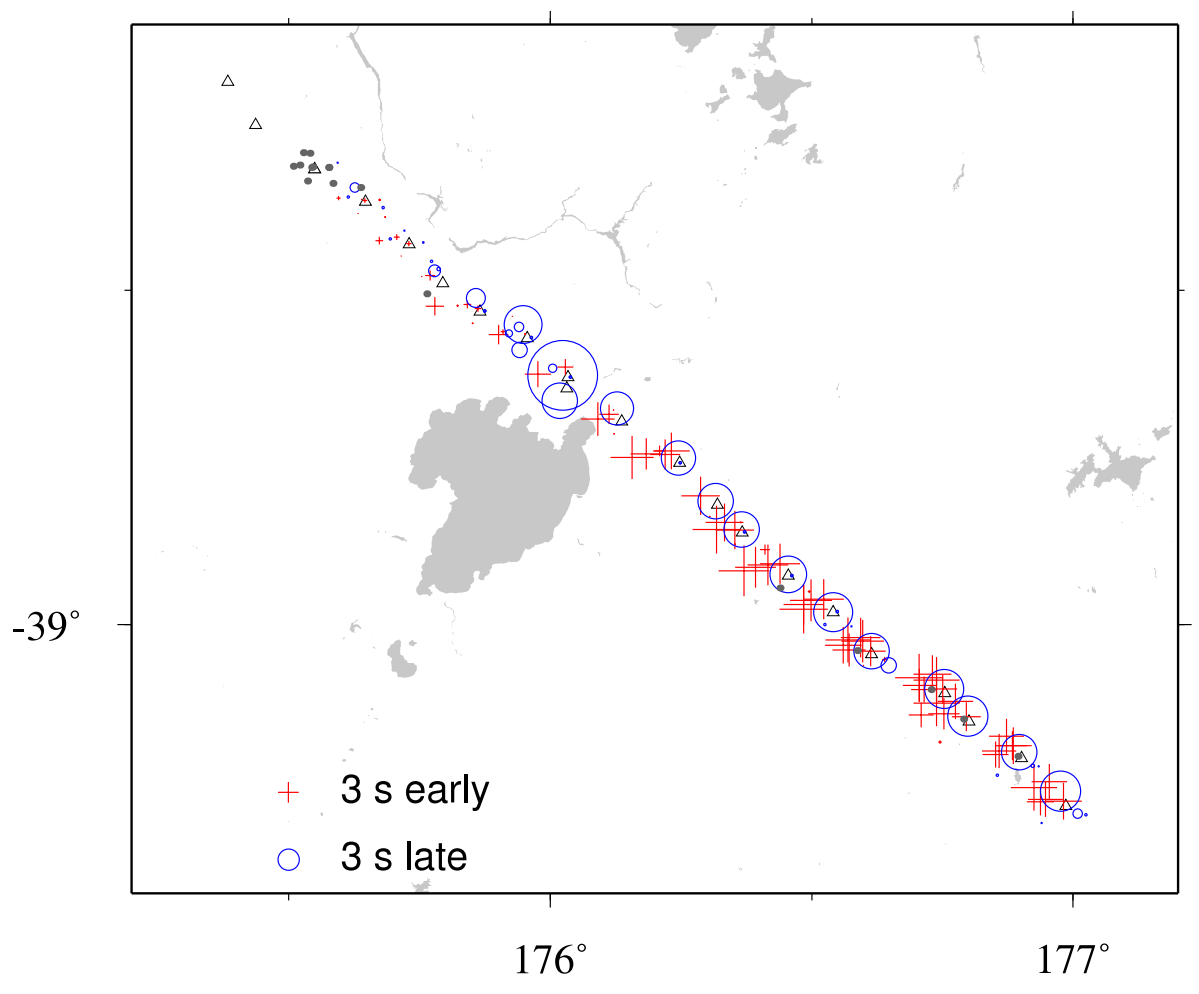

(b) S-phases (north-south component)

FIGURE 9.5 Map view of travel time residuals for P-and S-waves. Circles indicate late arrivals, crosses early arrivals. The size of the symbols is proportional to the travel time residuals. Symbols are plotted in direction of the backazimuth of the event relative to the station. The distance to the station is proportional to the incidence angle. Vertical incidence (angle $=0^{\circ}$ ) plots directly on the stations location; increasing incidence angles plot further away from the station. 


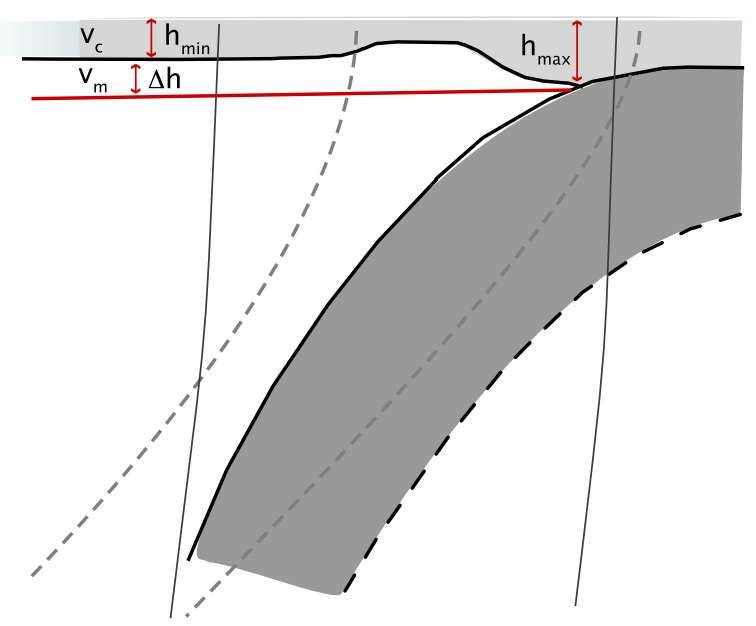

FIGURE 9.6 Cartoon of cross-section for central North Island showing different structural changes sampled by possible raypaths. Grey dashed lines are travel paths as suggested by Robinson [1976] and expected for shallow incidence angles, grey solid lines are travel paths assumed for distant teleseismic events. The parameters defined to calculate crustal corrections (equation 9.1) are: crustal $v_{c}$ and mantle velocity $v_{m}$, two different crustal thicknesses $h_{\min }$ and $h_{\max }$ and their difference $\Delta h$.

from variation of the travel time residuals with incidence angles (figure 9.5). Similarity of pattern at closely located stations indicates relatively deep anomalies, whereas abrupt changes between close stations are more likely to be caused by shallower structures [Polet, 2007]. Changes in travel time $\Delta t$ due to variations in crustal thickness $\Delta h$ (figure 9.6) can be estimated as:

$$
\Delta t=\frac{\Delta h}{\cos i}\left(\frac{1}{v_{c}}-\frac{1}{v_{m}}\right),
$$

where $v_{m}$ and $v_{c}$ are mantle and crustal velocity, respectively and $i$ is the incidence angle (for details see appendix H.2). For P wave crustal velocities of $4.5 \mathrm{~km} / \mathrm{s}$, mantle velocities of $7.9 \mathrm{~km} / \mathrm{s}$ [Stratford \& Stern, 2006] and a thickness difference of $10 \mathrm{~km}$ this lead to about $1 \mathrm{~s}$ travel time difference (for vertical incidence). This is on the order of station delay times between eastern and western central North Island of about $1.3 \mathrm{~s}$ found for Pn-phases [Seward et al., 2008], with shorter times in the west than in the east. This suggests that crustal variations are not sufficient to account for the total of the about $4.5 \mathrm{~s}$ observed P-wave travel time residuals. As travel time residuals integrate over all the anomalies along the travel path, most of the anomaly must be along deeper parts of the travel path, i.e. in the mantle.

Another structural factor influencing travel time residuals across a subduction zone is the subducting slab. It was suggested [e.g. Robinson, 1976] that phases arriving from the west travel through the high velocity slab and are therefore faster. This is certainly true for local shallow-incidence events, which can be guided through the high velocity region, but is not expected for deeper or more distant events [Prasad \& Bock, 1987]. Indeed, shallower incidences show larger travel time residuals (figure 9.5), even east of the CVR. This supports the idea higher velocity Pacific lithosphere [Galea, 1993].

With values over $7 \mathrm{~s}$, relative travel time residuals along the CNIPSE line are high, but 


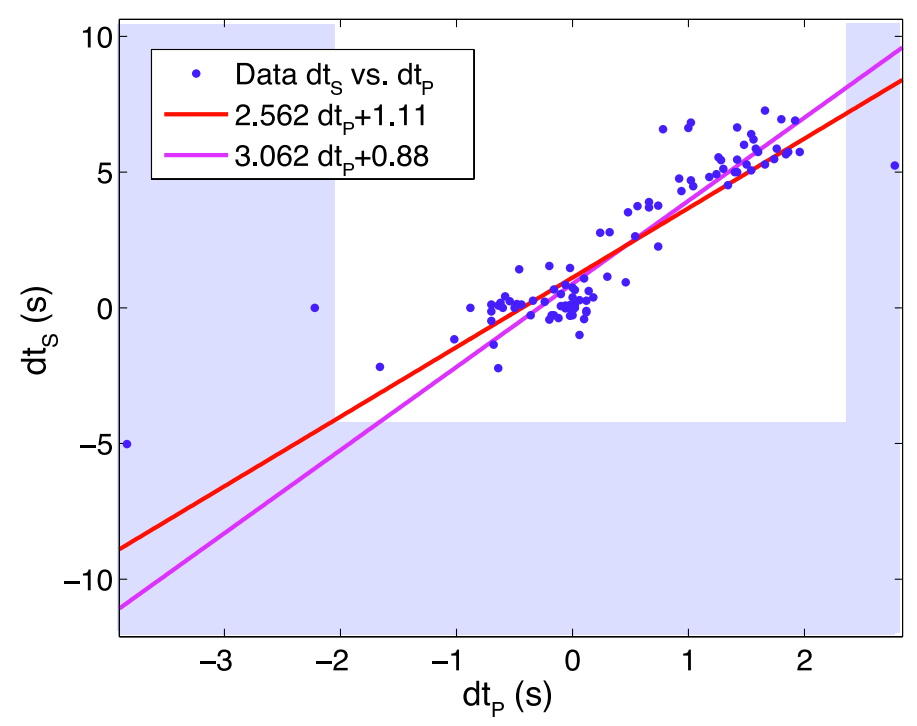

FIGURE 9.7 S/P delay time ratio and best linear fit for all data (red) and data excluding outliers (pink), located in the shaded area.

lower than the $16 \mathrm{~s}$ found east in the southwest Pacific region [Galea, 1993]. Comparable values are found in the Tien Shan shield, with P-wave travel time residuals of more than $5 \mathrm{~s}$ [Martynov et al., 2004]. Some studies in rift or subduction regions only find travel time residuals around 1-2 s [e.g. Cornwell et al., 2004; Polet, 2007], comparable with more stable regions [Guilbert et al., 1996]. Even though the corrections discussed above can account for parts of these high delay times, additional causes are needed to explain all the travel time residuals, especially the large delay times.

\subsubsection{S/P ratio}

For events that yield results for both, $\mathrm{P}$ - and S-wave travel time residuals, the two values can be compared. Differences in travel time residuals for P- and S-phases contain information about compositional and temperature anomalies along the travel paths. The ratio of the logarithmic derivatives of S- and P-velocity variations, often called the fractional change of velocities, is a value derived from tomography and commonly used in mineral physics. It can be expressed as [e.g. Masters et al., 2000]:

$$
\frac{d t_{S}}{d t_{P}} \approx \frac{v_{P} d \ln v_{S}}{v_{S} d \ln v_{P}}
$$

Therefore, the change in S-wave delay time versus P-wave delay time (figure 9.7) gives a handle on the fractional change in velocities. The best linear fit for the complete data set gives $d t_{S} / d t_{P}=2.562 \pm 1.265$ and an intercept of 1.111. After exclusion of some outliers (shaded area in figure 9.7), the fit is enhanced to $d t_{S} / d t_{P}=3.062 \pm 1.036$ and an intercept of 0.8814. This slope is comparable with global values found by Bolton \& Masters [2001]. They find 3.1 for shallow turning waves $(670-1100 \mathrm{~km})$, deeper turning waves lead to higher values up to 7.2. This verifies the upper mantle as the main source for the travel time residuals. The offsets of the best-fit line ( $y$-axis intercept) can be explained by slower average S-wave 
velocity in the study region compared to the reference model. However, results are poorly constrained and data broadly scattered.

As is evident from equation 9.2 , knowledge of the $v_{P} / v_{S}$-ratio is necessary to convert the observed S/P ratio from travel time residuals into the ratio of the logarithmic derivatives. Taking an average upper mantle $v_{P} / v_{S}$-ratio of 1.81 from the ak135 reference model [Kennett et al., 1995] leads to $d \ln v_{S} / d \ln v_{P}$ of 1.41 or 1.69 for the full dataset with and without outliers, respectively.

Seismological derived fractional velocity changes for the upper mantle show a preponderance of $d \ln v_{S} / d \ln v_{P}=1.7$ [e.g. Robertson \& Woodhouse, 1996; Bolton \& Masters, 2001; Schmid et al., 2004]. Cammarano et al. [2003] calculate values for the upper mantle between 1.3 and 2.2, overlapping with the range of seismological observations (figure 9.8). They state that the values are more sensitive to temperature changes than to compositional changes. This agrees with Isaak et al. [1992], who conclude that temperature and pressure effects can explain logarithmic ratios up to about 2.5. Hammond \& Humphreys [2000] conclude that fractional velocity changes between 1.2 to 1.3 are an indicator for temperature anomalies, whereas high values around 2.2 indicate the presence

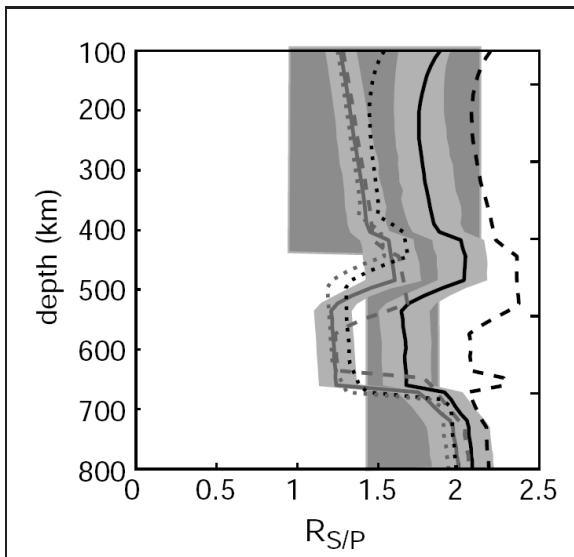

FIGURE 9.8 Calculated fractional velocity changes, here called $R_{S / P}$, for different adiabats (lines), compared to seismological observations (dark grey shaded areas), figure from Cammarano et al. [2003]. of partial melt.

In the attempt to look for regional variations, data are split up into the three distinct regions identified in the anisotropy analysis (cf. figure G.1, table G.1). This leads to slopes of $d t_{S} / d t_{P}=1.738 \pm 0.8993$ for the CVR and $d t_{S} / d t_{P}=2.555 \pm 1.289$ for eastern North Island (figure 9.9). There is not sufficient data for western part of the CNIPSE line to get results for western North Island. The values obtained lead to logarithmic ratios of $d \ln v_{S} / d \ln v_{P}=0.96$ for the CVR and $d \ln v_{S} / d \ln v_{P}=1.41$ for eastern North Island. These values agree with fractional changes in velocities derived from local earthquake tomography [Reyners et al., 2006] of about 1 in the backarc and 1.3 the arc region. Such low logarithmic ratios are characteristic for texturally equilibrated partially molten rock [Takei, 2002]. However, the values depend on the aspect ratio of the melt inclusion. Logarithmic ratios of 1 to 1.7 correspond to aspect ratios from 0.1 to 0.01 . Melt inclusions with these aspect ratios yield anisotropy of 10 to $20 \%$ ( $c f$. section 7.6.1) and are sufficient to explain the observed high anisotropy in the CVR (cf. section 5.4). Lower aspect ratios $\left(\sim 10^{-3}\right)$ give values up to 2.25 .

Additional evidence for anomalous $v_{P} / v_{S}$-ratios comes from active source seismic studies. Ray-tracing to fit first P-arrivals results in large residuals for S-phases (up to $2.4 \mathrm{~s}$ ) in the area under and west of the TVZ [Harrison \& White, 2006]. Only the inclusion of anoma- 


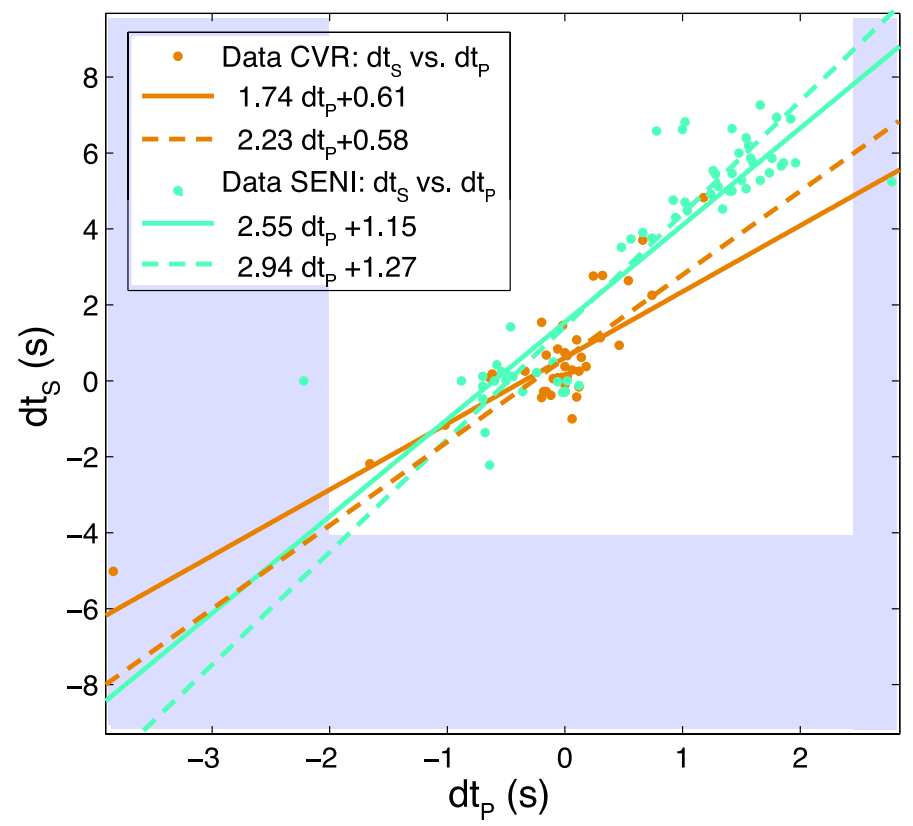

FIGURE 9.9 Regional S/P delay time ratio, for CVR (orange) and eastern North Island (blue). Data is shown as points, solid lines show best linear fit, dashed line is best fit excluding outliers in shaded area.

lously high Poisson's ratios allows matching of first arrivals for both phases.

Temperature changes and the presence of melt and water have a strong influence on the fractional change in velocities. In the subduction zone setting, both factors will account for parts of the observed velocity decrease ratios [Nakajima et al., 2005]. Further investigations are needed to distinguish the exact influence of temperature and the percentage and aspect ratio of partial melt.

\subsubsection{Anisotropic velocities}

Previous interpretations (sections 9.4.1 and 9.4.2) are based on the assumption of isotropic subsurface structure. Under this assumption, travel time residuals can be used to produce traditional tomographic depth images. But information about directional variations is lost in tomography. Working with travel time residuals has the advantage of displaying the effect of backazimuthal variations, which provide important information on P- and S-wave average isotropic, as well as anisotropic seismic properties.

Backazimuthal variations in travel time residuals are observed in the CNIPSE data set. Even though backazimuthal variations include the effects of inhomogeneities elsewhere along the ray path, consistent variations across an array can be related to local structure beneath the stations. Westerly arrivals give negative relative residuals, decreasing from west to east. Easterly events give positive relative travel time residuals, increasing from west to east. This implies an increased apparent velocity for waves arriving from west to northwest. Increasing velocities from west to east for events arriving from northwest were noticed before [Robinson, 1976] at fourteen permanent stations on North Island. They were interpreted as due to the influence of the higher velocity slab, acting as a waveguide. However, for teleseismic events with steep incidence angles a predominant travel path through 
the slab is not expected (figure 9.6). Still, a slightly longer travel path for westerly phases can be explained through the westward dip of the slab. Travel time residuals for waves arriving from easterly directions, in contrast, are more consistent, as travel path lengths through the slab to stations along the array for these phases are more equal. Details about the exact ray path depend on a combination of parameters such as incidence angle, slab dip and length and velocity contrast, and can only be determined with ray tracing.

Directional variation of P-wave travel time residuals with event backazimuth can be an indicator for anisotropy. Therefore, the analysis of travel time residuals often accompanies and expands shear-wave splitting measurements [Babuška et al., 2002; Plomerová et al., 2006; Sileny \& Plomerová, 1996; Vecsey et al., 2007; Bokelmann \& Silver, 2000]. When comparing both techniques, it is important to consider the different nature of P- and S-waves. Teleseismic P-wave travel time residuals are not affected by anisotropy with horizontal symmetry, as they travel almost vertically and are polarised along the travel path. But dipping symmetry can have strong effects. Therefore, teleseismic P-wave travel time residuals can be an indicator of dipping symmetry axis. However, modelling of the observed shear-wave splitting did not favour dipping axes for the main part of the travel path (chapter G.2). If dipping axes can be excluded, the variations must be related to isotropic velocity changes. On the other hand, a thin dipping anisotropic layer on top of the slab (e.g. McKenzie [1979]) might not be seen in teleseismic shear-wave splitting, but could influence local phase arrival times. In contrast to teleseismic P-waves, shallow incidence local P-waves (figure 9.6) are more strongly influenced by anisotropic structure with horizontal symmetry axis. This could explain higher travel time residuals with shallower incidence angles (figure 9.5).

In the CVR, the region with the strongest changes in travel time residuals, shear-wave splitting measurements also show strong anisotropy (chapter 5). In warm regions, with low velocities and high anisotropy, P-delays and splitting are expected to be positively correlated [Schmid et al., 2004]. This is confirmed by tomography results, where changes in velocities correlate well with changes in anisotropy and large delay times correlate with low velocities [Gök et al., 2003]. But, in the presence of anisotropy, waveforms get distorted (as the fast and slow component overlap), which complicates measuring travel time residuals and increases errors. Further to the east, shear-wave splitting presents a more average, consistent anisotropy. This correlates with high, slowly increasing travel time residuals in the eastern Central North Island. Tomography yields elevated $v_{P} / v_{S}$ ratios under the CVR, and more average values under eastern North Island (cf. figure 5.6) [Reyners et al., 2006].

P- and S-phases from westerly backazimuths, almost perpendicular to the trench, arrive earlier than from other backazimuths (figures 9.4 and 9.5). This observation is not consistent with a cause due to anisotropy observed in central North Island. Moreover, it contradicts trench-parallel fast polarisation found from shear-wave splitting (chapter 5.4) of teleseismic events. However, local events give trench-perpendicular fast polarisation in the CVR, which fits in better with advanced arrivals from the west. The differences in fast polarisations found from local and teleseismic waves is suggested to be due to frequency dependence 
caused by melt-filled inclusions (section 5.5.2). Martynov et al. [2004] also find contradictions between fast polarisations determined from travel time residuals of P-waves and shearwave splitting and suggest frequency dependence as a possible explanation. Anisotropy caused by shape-preferred orientation and probably also slab anisotropy show horizontal symmetry axes. This could help to explain the stronger effect of the backazimuthal dependence on the S-phases and the higher delays for shallower P-waves. Additionally, structural variations probably contribute to observations, as mentioned above ( $c f$. section 9.4.1)

\subsection{Conclusion and Outlook}

The observed directional changes in travel time residuals together with possible regional differences motivated a more detailed shear-wave splitting study across the CNIPSE array (chapter 5) including new data from its western extension (section 4.1.3). This track then became a study in itself. A more complete study of travel time residuals will be a powerful addition to mantle structure in central North Island.

The preliminary results discussed give evidence for lateral structural and velocity changes (section 9.4.1), fluid and/or melt (section 9.4.2) and anisotropy (section 9.4.3). The exact contribution from each of these causes to the total of the observed travel time residuals cannot be clarified at this stage and need to be examined in a future study.

The future study would profit from the vast data set available now for central North Island, including the WCNIPSE temporary deployment and data from the permanent national network (GeoNet). A more detailed travel time residual study could help to enhance the velocity model of central North Island by testing and correcting existing crustal [Stratford \& Stern, 2006; Harrison \& White, 2006] and mantle [Reyners et al., 2006] velocity models. Furthermore, a more thorough evaluation of the velocity decrease ratios can give information about temperature, presence of partial melt and aspect ratios of inclusions. Scale length of heterogeneities can be estimated by the additional evaluation of frequency dependence of travel time residuals [Schmid et al., 2004]. Finally, to extend the joint analysis of travel time residuals and shear-wave splitting, an anisotropic tomography study could be attempted [e.g. Eberhart-Phillips \& Henderson, 2004].

Improvements to the processing method suggested here can be achieved through better application of corrections. Furthermore, S-wave travel time residuals can be evaluated on the transverse component, to reduce the influence of waveform distortion from converted phases. Additional advances in the evaluation of travel time residuals could result from reducing the correlation window around the phase onset. The scatter in the data caused large uncertainties in the determination of the fractional changes in velocities (section 9.4.2). A more extended data set is necessary to constrain results and to look for regional variations in $\mathrm{S} / \mathrm{P}$ ratios.

An enhanced evaluation of the exact travel paths for the different phases is necessary for 
a more detailed interpretation of the source of the observed travel time residuals. The finite difference model used to generate synthetic waveforms for shear-wave splitting (section 6.2) could be improved by adjusting velocities and structural details to more concrete values for central North Island [Stratford \& Stern, 2006; Harrison \& White, 2006; Reyners et al., 2006]. This would allow for a combined model of travel time residuals and anisotropy and provide better constraints for model parameters. The elastic tensors needed for the new model can be derived by Reuss-Hill-averaging (chapter 3) and analytical modelling, as described in chapter 7. Additional results from other methods such as Pn studies, indicating azimuthal variations in velocity, and receiver functions, imaging conversion interfaces (seismic velocity discontinuities), can accompany findings and further constrain the upper mantle model of the complex subduction zone setting under central North Island. 


\section{Chapter 10}

\section{Conclusions}

This thesis investigates relations between anisotropy, subduction zone dynamics and the presence of melt in the Hikurangi subduction zone, central North Island, New Zealand. In particular, details about the anisotropic structure and characteristics have been identified using shear-wave splitting measurements, analytical and finite difference modelling, and different methods to evaluate frequency dependent anisotropy. The principal findings of this study are summarised as follows:

- Strong lateral anisotropic changes across the Hikurangi arc setting in central North Island are identified by shear-wave splitting measurements. The observations divide the central North Island into three distinct anisotropic regions: 1.) The forearc region, eastern North Island, with shear-wave splitting measurements consistent with southern North Island; trench-parallel fast polarisation for teleseismic and local shear-waves and delay times around 2 to $2.5 \mathrm{~s}$ for teleseismic. 2.) The arc/backarc region, Central Volcanic Region (CVR), with anomalous high delay times of up to $4.5 \mathrm{~s}$ and trenchparallel fast polarisation from teleseismic phases. 3.) The far backarc region, western North Island, with no apparent anisotropy identified from teleseismic phases.

These regions of observed changes in anisotropy correlate well with other changes in geophysical properties occurring in the CVR and western North Island such as in gravity, attenuation, velocity $(\mathrm{S}, \mathrm{Pn})$ and heat flow. These changes are attributed mainly to the influence of the hot, melt- and volatile-rich mantle wedge under the CVR and small-scale convection processes or vertical flow under the western North Island, probably caused by lithospheric delamination of the upper mantle. The western boundary of the CVR marks a change between high anisotropy in the mantle wedge and apparent isotropy under western North Island. Trench-parallel subslab mantle flow contributes about 2-3 s of delay times (as observed in the forearc region) to the overall anisotropy. Contributions of fossil slab anisotropy are possible.

- The two-dimensional finite difference modelling applied to the anisotropic upper mantle structure under the central North Island proves to be a sufficient tool to test sug- 
gested interpretations for shear-wave splitting measurements. The possibility of defining different anisotropic domains without the restrictions of horizontal layers allows us to incorporate a realistic model of the Hikurangi subduction zone. The full wave propagation from different backazimuths takes effects such as Fresnel zone sampling and wavefront healing into account.

The preferred model determined by the finite difference method explains the shearwave splitting observations across the CNIPSE line with a depth structure comprising four domains of very different anisotropic character: an isotropic backarc region under western North Island, a highly anisotropic ( $10 \%)$ mantle wedge under the CVR, an isotropic slab and a subslab mantle with average anisotropy (3.5\%). All anisotropic regions have trench-parallel fast orientations. While the model supports most of the main interpretations argued before, such as the high anisotropy in the mantle wedge and the apparent isotropy in the far backarc, it excludes the idea of considerable anisotropy in the slab.

- The presence of melt in the mantle wedge can account for two of the outstanding anisotropic characteristics of central North Island: the large teleseismic delay times and a frequency dependence of shear-wave splitting parameters. The various smallscale analytical approaches to model melt inclusions in mantle materials show that small percentages of melt (about $2 \%$ ) are sufficient to account for the strong anisotropy needed in the mantle wedge below the CVR. The modelling results put a constraint on the properties that are realistic in the presence of melt and justify the up to $10 \%$ anisotropy used in the large-scale finite difference models. However, the complexity of models prevents more detailed interpretation in consideration not to over-interpret the data.

The geometry of the melt inclusion is an important factor for the strength of the derived anisotropy, but is still an unknown parameter for partial melt occurrence in the mantle wedge. However, the presence of connected pathways such as pores, fractures or dykes is required to allow melt to surface at active volcanoes. Connectivity plays a significant role for the generation of frequency dependent effects. The applied frequency dependent model requires aligned large-scale heterogeneities on the order of tens of metres to match observed changes in the local and teleseismic frequency ranges, but the cause of the discrepancy remains unsettled.

- The proposed frequency dependence is difficult to establish for central North Island. Teleseismic events proved not to be ideal for a detailed, systematic frequency dependent analysis, as the frequency range covered is not broad enough to overlap with the frequency range of local events. Furthermore, it is expected that the large Fresnel zone of the low frequency teleseismic waves smear out regional frequency dependent effects, such as suggested for the CVR. However, considering the complete North Island data set, a general trend towards smaller delay times for higher frequency is observed. This trend is fitted with a gradient of about $-3 \mathrm{~s} / \mathrm{Hz}$. Fast polarisations stay constant 
over the analysed frequency range. This is consistent with the presence of a single preferred inclusion orientation. Nonetheless, the fact that fast polarisations for teleseismic and local shear-wave splitting measurements are perpendicular can be considered as frequency dependence, caused by the presence of two preferred inclusion orientations of different scale. The difference in scales is inferred to be on the order of more than a magnitude, in accordance with the frequency difference. Thus, with the scale length from the delay times determined to be about tens of metres, the smaller scale cracks should be less than the metre scale.

Aligned large-scale melt-rich inclusions provide a suitable explanation for frequency dependent anisotropy across central North Island. They need to occur in steeply dipping planes to affect vertically travelling shear-waves. The high volcanic productivity of the TVZ could be the result of the effective melt transport provided by such meltrich channels or dykes. Shear-strain could support the development of these melt-rich layers, which in turn, could support the active rifting in the backarc region of the Hikurangi subduction zone.

Overall, this study stresses the importance of considering different scale heterogeneities for a comprehensive understanding of seismic anisotropy. Direct extrapolations from measurements on hand samples in the lab to field observations on much larger scale are not always valid and intermediate mesoscale patterns need to be incorporated into interpretations. Particularly in the presence of melt, microscale measurements are no longer sufficient to describe the overall anisotropy of the structures as they are sampled by teleseismic shear-waves. Differences in mesoscale melt geometry (e.g. dykes, channels) could provide an additional explanation for the large variations in subduction zone anisotropy observed worldwide.

The preliminary study on travel time residuals identified interesting pattern across the Hikurangi subduction zone. Travel time residuals reach high values (up to $10 \mathrm{~s}$ ) and display backazimuthal variations. The tentative interpretation shows ways to examine these results for information about the velocity structure. Moreover, the differences in P-and S-waves can yield valuable information on melt distribution and geometry. This establishes the study of travel time residuals as suitable addition to the shear-wave splitting study and suggests a more detailed examination of travel time residuals across the CVR as a future project.

Several additional opportunities for future research emerged during the course of this study. They mainly refer to the extension of the powerful finite difference method for anisotropic wave propagation:

- The introduced analytical modelling approaches provide full synthetic tensors for media with melt inclusions. These could be incorporated into the finite difference modelling for an even more realistic model set up, which might further improve results.

- To exploit the full potential of the finite difference model, the preferred model should be extended into the slab-parallel third dimension. This would allow the inclusion of 
the large amount of shear-wave splitting measurement from the lower North Island into the modelling. Moreover, actual results from other North Island studies, such as structure determined from receiver functions, tomography and active source surveys, and percentage anisotropy from local shear-wave splitting could be implemented.

- Hydrous phases such as chlorite and serpentinite are highly anisotropic [Mainprice, 2007]. However, their influence on subduction zone anisotropy is only slowly becoming recognised [e.g. Kneller et al., 2008] and is still poorly understood. The inclusion of hydrous regions could further refine the finite difference model structure.

- Finally, the systematic frequency dependent analysis should also be applied to local phases recorded on broadband stations. This could provide valuable information about frequency dependent effects in the missing intermediate frequency range between teleseismic and local phases. 


\section{APPENDIX A}

\section{Elastic tensors}

The following table gives some published full elastic tensors for mantle material, as they can be used in the finite difference code [Boyd, 2006]. The first olivine tensor is the one provided with the code, and has been used in the modelling in chapter 6.2 to describe average olivine properties. The first xenolith is the North Island xenolith found near Raglan, with properties derived by Duclos [2005]. The references cited are:

1 - Duclos [2005]

2 - Boyd [2006]

3- Abramson et al. [1997]

4 - Bascou et al. [2001]

5 - Ben-Ismail et al. [2001]

6 - Chai et al. [1997]

7 - Pera et al. [2003]

8 - Ismail \& Mainprice [1998]

9 - Vauchez et al. [2005]

10 - Collins \& Brown [1998]

11 - Kumazawa \& Anderson [1969]

12 - Mainprice \& Silver [1993]

13 - Zhang \& Karato [1995]

14 - Kumazawa [1969] 


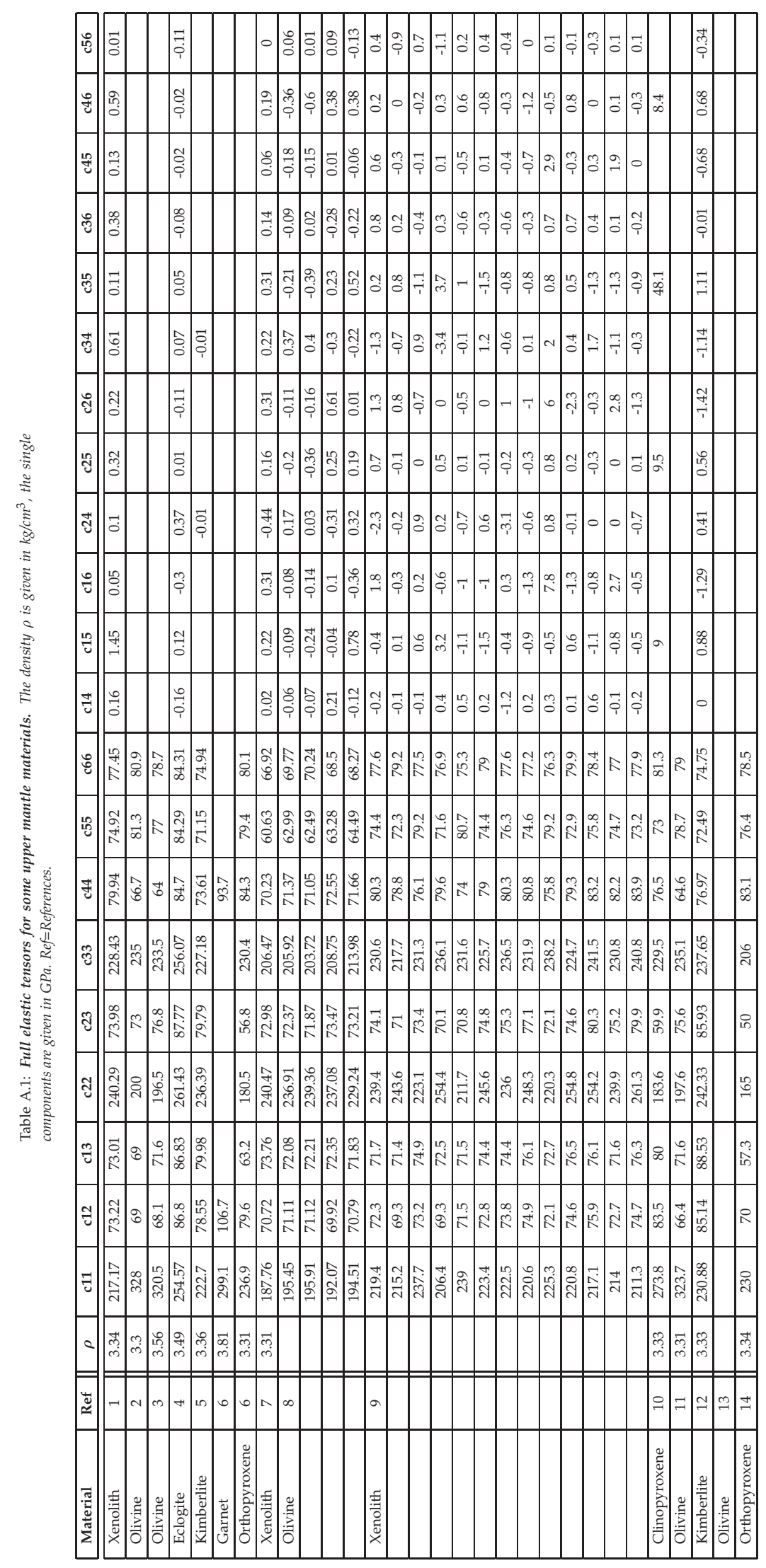


APPENDIX B

Station details 


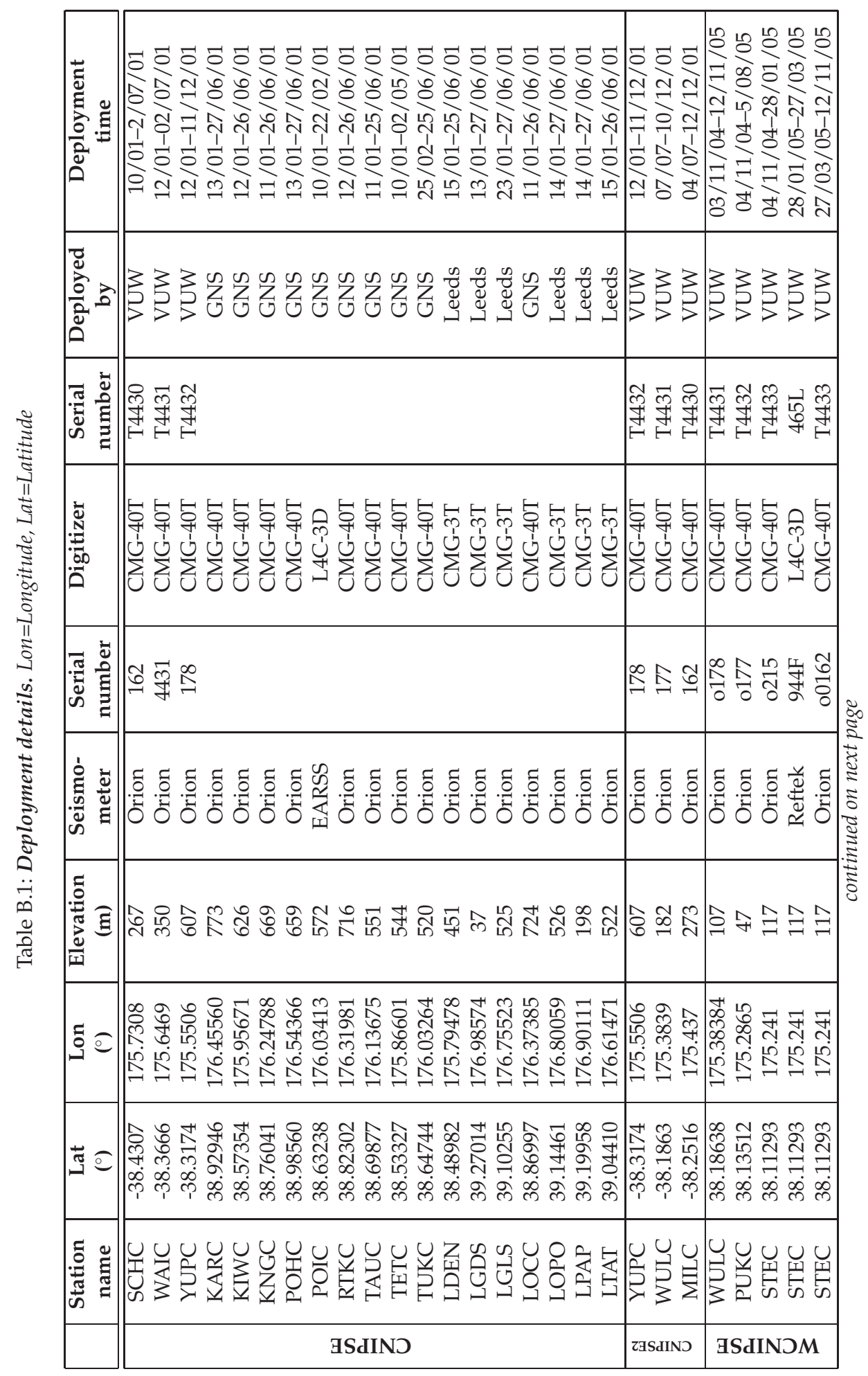




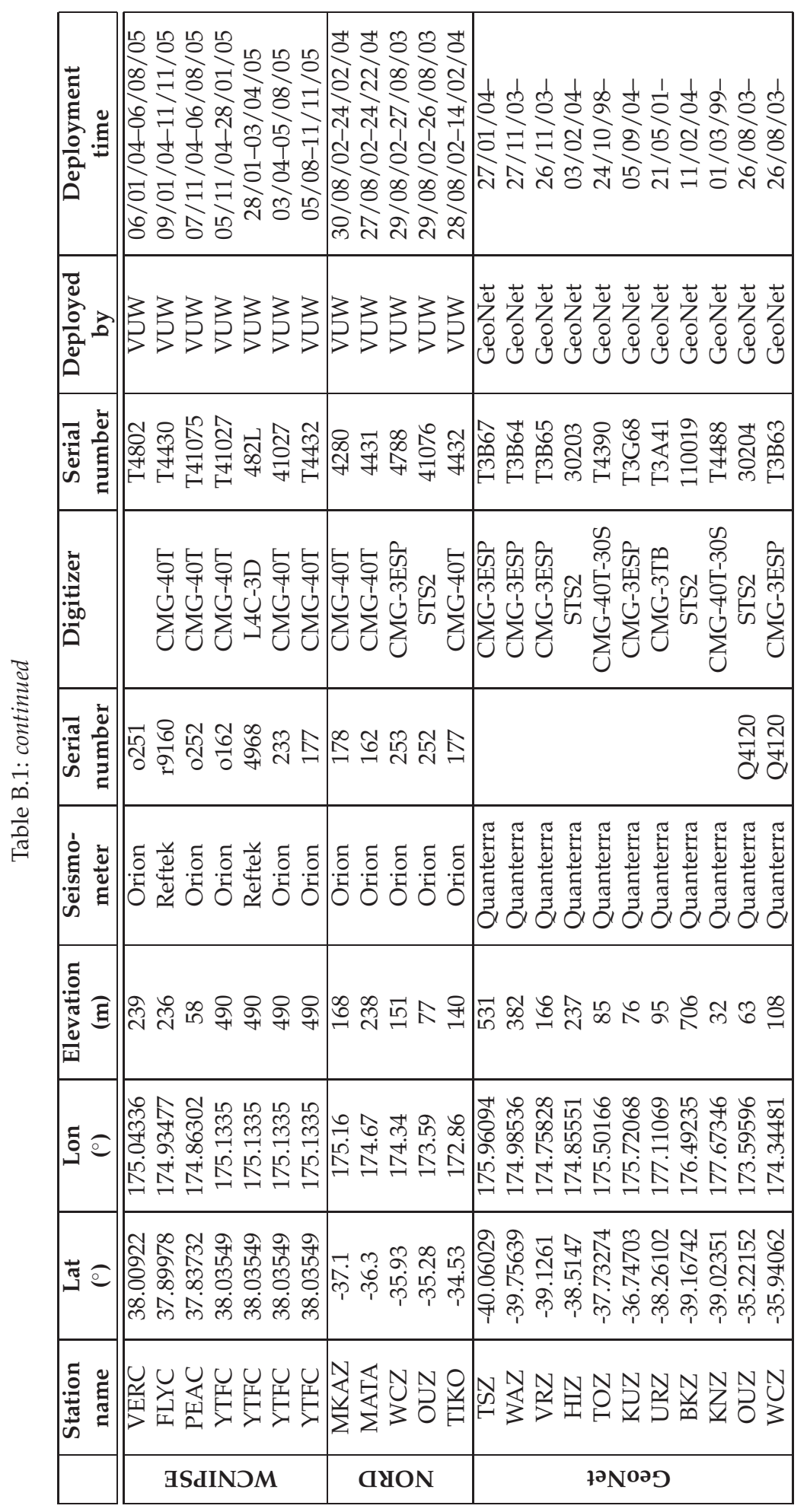




\section{APPENDIX C}

\section{Processing details}

This chapter provides an introduction into the raw data processing. A manual with full details about all the single processing steps was assembled during the course of this thesis and is attached as a supplement.

\section{C.1 ORION processing}

The standard Orion processing starts with downloading the data from the disk via a SCSI port onto a PC system. There, data get decompressed using the DOS program "rbtrim". Then, the directory called "USER" needs to be changed to a name unique to the station following the structure: $\mathrm{O}<$ ORION-number $>\mathrm{D}<$ DISK-number $><$ Station-name $>$. As the main preprocessing routines are based on UNIX software, the whole directory is then copied onto a UNIX system. All downloads from the different stations should be located in the same (experiment) directory.

The first processing routine to be used (" $m$ kresponse") creates response files (cf. section 4.2.2 and appendix C.3). Then, state of health information and station locations are extracted and placed in the experiment directory using the program "summarize". Data extraction can be done in three ways. The program "extractall" extracts the complete data set and combines 24-hour blocks into SEED data format in a directory called "daily". The program "extractlocal" extracts local events triggered by the internal Orion trigger. However, the way to extract the desired data is to use earthquake catalogue data. Local events are taken from GeoNet (www.geonet.org.nz), recent teleseismic events from IRIS

(www.iris.washington.edu/pub/HYPOS/QED_daily) and older teleseismic events from ISC (www.isc.ac.uk), because of revised locations and timing. The event catalogues need to be converted in a format suitable for the Orion software. Then, the events to be extracted are selected depending on their distance and magnitude and combined into "blocks" (table 4.1. This is done using the program "weed". The program "extractsummary.pl" then creates summary files and extracts the event data into SEED files. For each block, the SEED files are 
moved into a corresponding subfolder of a directory called "global".

\section{C.2 REF TEK processing}

Raw data get downloaded onto the computer system into a data directory named after the REF TEK DAS number. The first thing to do is running "rt130cut" to merge and format all the single data files. One single (".ref") data file for each DAS is created. Then data can be converted into $\mathrm{m}$ (ini)seed format using "ref2mseed". Once the data files are generated, the timing should be checked for the recorded data. This is done with the Passcal routine "refrate" using the ".log"-file. The output is a ".pcf"-file, which will later be used to correct for timing errors. There are two options to correct the timing: correcting the full data file using "clockcor" or correcting single events while extracting earthquakes using the "-c" option in "eqcut". Because of these two options it is important to ensure to apply the REF TEK clock correction only once to any given file. A Passcal routine called "position" calculates station locations from the GPS logs. Station locations can be included into the headers later. Mseed data can only contain four header values: time, station, network and channel. To list them, use "mseedhdr"; to edit them, use "mseedmod".

The next step is to cut data and extract events using "eqcut". The routine uses input files and can therefore cut many events in one single process. A UNIX shell script to create these input files is "mkeqcut", which needs an event list in the same format as "weed" in the Orion processing uses. It will sort these events in different blocks according to their magnitude and distance (table 4.1). The outputs are event files for each block and DAS and a DAS file to be used by eqcut. In the last step the cut event data get converted into SAC format, using "ms2sac". In this step the station locations are included into the headers.

\section{C.3 Instrument correction details}

To create the files containing the instrument response for Orions, the routine "mkresponse" is generally used. It is based on another routine called "response", which is available from Nanometrics. "mkresponse" modifies the general response files created by "response" in four places: name of response file, sign of normalisation factor in stage 1, gain or sensitivity in stage 1, and input sampling rate. These changes are based on an input file named after the serial number of the sensor, e.g. T4430.BBE.corr, containing all the instrument specific data. Currently, in the VUW system, these files only exist for sensors number: T4430, T4431, T4432, T4433, T4788, T4C49 and T41076. Therefore, the standard preprocessing only works for these sensors.

If the standard processing cannot be applied, the response files need to be created manually. In this case it is best to use "mkresponse" to create a default response file and alter the following: 
1. The name of the default file (default.BBE.rsp) should be changed corresponding to the sensor serial number (e.g. T4433.BBE.rsp), also in the file itself.

2. The normalisation factor $A_{0}$ (normalised to 1 ) has to be changed for stage 1 , which describes the sensor (seismometer) and is the same for all three components.

3. The sensitivity or gain in stage 1 (seismometer sensitivity) has to be in units of $\mathrm{V} / \mathrm{m} / \mathrm{s}$ (velocity output) and is different for all three components. It can be found in the calibration sheet.

4. The sensitivity or gain in stage 2 describes the amplifier and is therefore just a constant factor and independent on the sensors. Its value for the Orions is 1.6. The gain or sensitivity in stage 3 describes the analogue lowpass filter of the digitiser (Orion) and can be calculated using the Orion sensitivity and the gain from stage 2:

$$
\operatorname{gain}(\text { stage })[V / \text { bits }]=\frac{1}{\text { Orion sensitivity }[V / \text { bits }] * \text { gain (stage } 2)[]}
$$

Values also differ for different components.

5. Poles and zeros have to be in units of rad/sec, if "type of stage" in the response file is specified as A. Multiplying by $2 \pi$ converts from $\mathrm{Hz}$ to rad/sec. As the filter in stage 2 is constant, they only need to be given in stage 1 for the sensor. 


\title{
APPENDIX D
}

\section{Data}

\author{
D.1 Event lists
}

D.1.1 Event used for shear-wave splitting analysis 


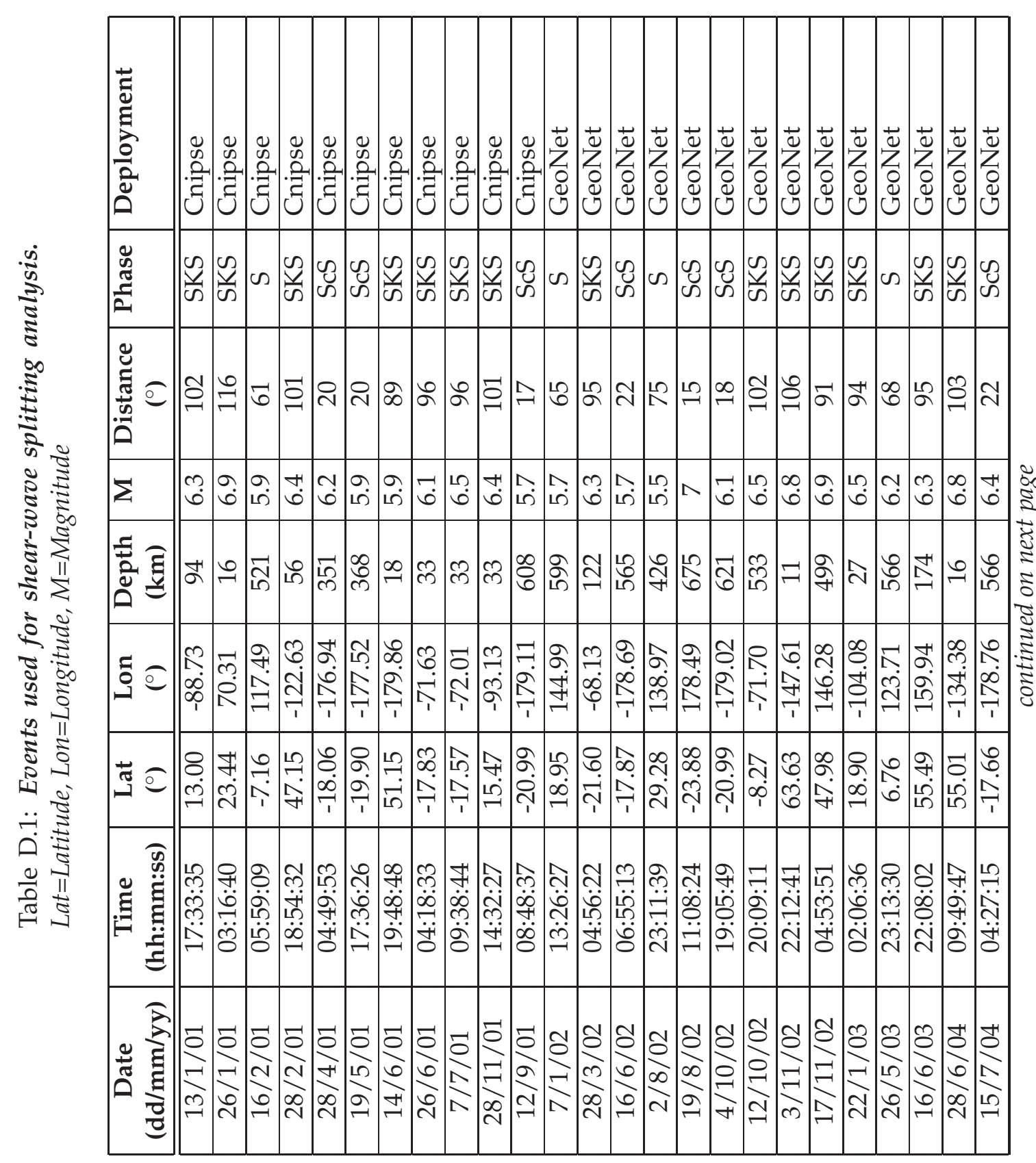




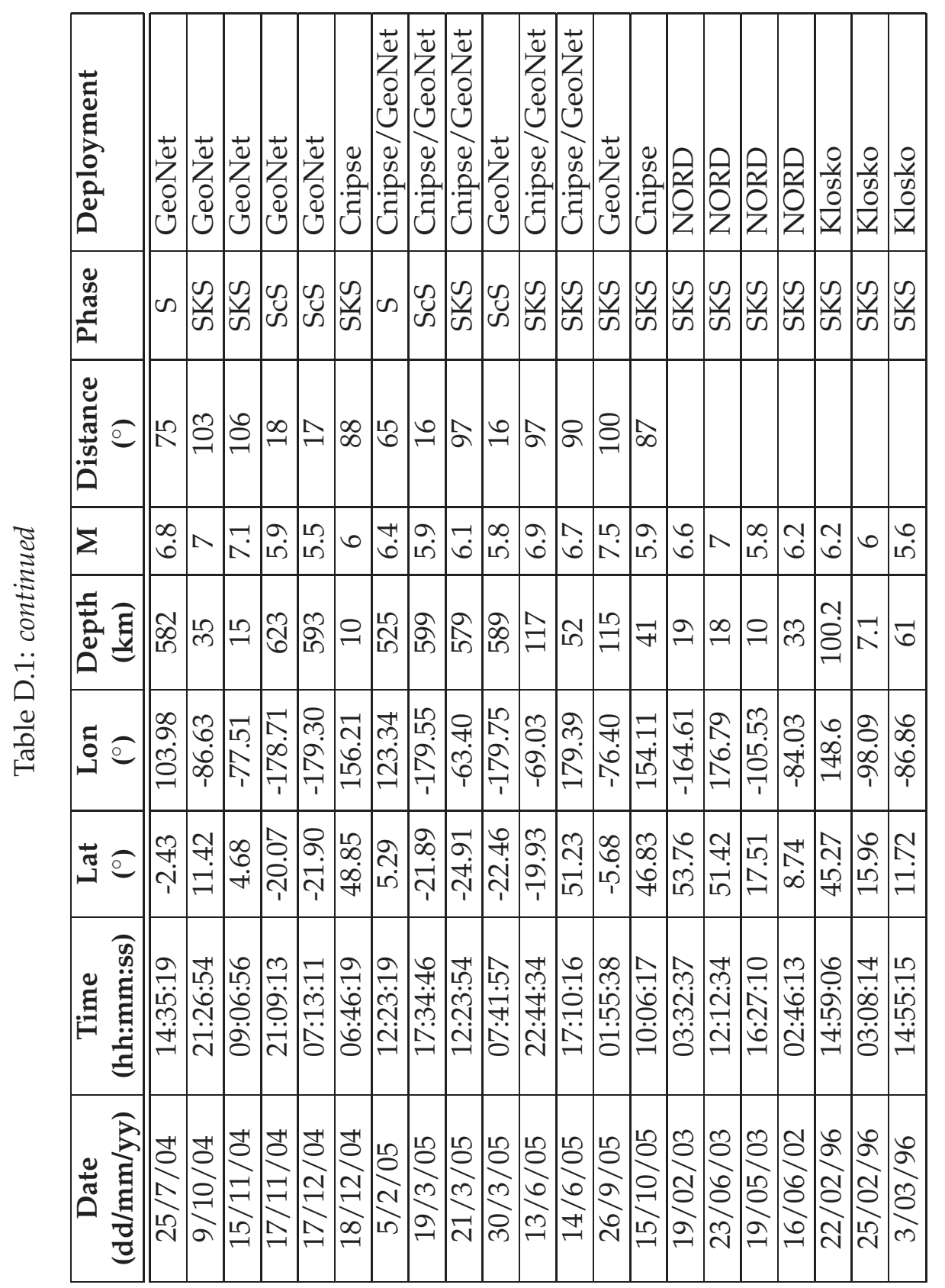




\section{D.1.2 Event used for travel time residuals analysis}

TABLE D.2 Events used for travel time residuals analysis. Lat=Latitude, Lon=Longitude, M=Magnitude

\begin{tabular}{|c|c|c|c|c|c|c|}
\hline $\begin{array}{c}\text { Date } \\
\text { (yyyy/mm/dd) }\end{array}$ & $\begin{array}{c}\text { Time } \\
\text { (hh:mm:ss) }\end{array}$ & $\begin{array}{l}\text { Lat } \\
\left({ }^{\circ}\right)\end{array}$ & $\begin{array}{c}\text { Lon } \\
\left({ }^{\circ}\right)\end{array}$ & $\begin{array}{c}\text { Depth } \\
(\mathrm{km})\end{array}$ & $\mathbf{M}$ & Phase \\
\hline $2001 / 01 / 13$ & 17:33:34.58 & 12.9970 & -88.7290 & 83 & 6.3 & $\mathrm{P}, \mathrm{S}$ \\
\hline $2001 / 01 / 14$ & 08:58:25.80 & 22.1050 & 143.8360 & 94 & 5.7 & $\mathrm{P}$ \\
\hline $2001 / 01 / 15$ & $05: 52: 15.80$ & -40.3380 & 78.3300 & 10 & 5.5 & $\mathrm{P}, \mathrm{S}$ \\
\hline $2001 / 01 / 19$ & 08:10:16.32 & -11.7050 & 166.3610 & 63 & 5.7 & $\mathrm{P}$ \\
\hline $2001 / 01 / 26$ & $03: 16: 40.26$ & 23.4420 & 70.3100 & 16 & 6.9 & $\mathrm{P}$ \\
\hline $2001 / 01 / 29$ & $23: 21: 25.62$ & -0.7170 & 133.2840 & 33 & 5.7 & $\mathrm{P}$ \\
\hline $2001 / 02 / 13$ & 19:28:30.48 & -4.7540 & 102.4780 & 33 & 6.2 & $\mathrm{P}, \mathrm{S}$ \\
\hline $2001 / 02 / 15$ & 12:47:00.7 & -5.583 & 148.309 & 178 & 5.7 & $\mathrm{P}$ \\
\hline $2001 / 02 / 16$ & 05:59:09.60 & -7.1380 & 117.4870 & 527 & 5.9 & $\mathrm{P}, \mathrm{S}$ \\
\hline $2001 / 02 / 24$ & $07: 23: 50.49$ & 1.3340 & 126.3350 & 56 & 6.5 & $\mathrm{P}$ \\
\hline $2001 / 02 / 28$ & 12:30:18.01 & -21.9920 & 170.1330 & 34 & 5.8 & $\mathrm{P}$ \\
\hline $2001 / 03 / 06$ & 09:17:36.42 & -54.5310 & 157.2770 & 10 & 5.8 & $\mathrm{P}, \mathrm{S}$ \\
\hline $2001 / 03 / 14$ & 18:56:21.69 & 0.3940 & 121.9230 & 135 & 5.7 & $\mathrm{P}$ \\
\hline 2001/03/15 & 13:02:44.00 & -32.3520 & -71.4810 & 53 & 6.1 & $\mathrm{P}$ \\
\hline $2001 / 03 / 24$ & 06:27:53.85 & 34.0950 & 132.5520 & 52 & 6.3 & $\mathrm{P}, \mathrm{S}$ \\
\hline 2001/04/09 & 09:00:57.28 & -32.7540 & -73.1500 & 10 & 6.1 & $\mathrm{P}, \mathrm{S}$ \\
\hline $2001 / 04 / 19$ & $21: 43: 42.56$ & -7.4100 & 155.8390 & 17 & 5.8 & $\mathrm{P}, \mathrm{S}$ \\
\hline $2001 / 04 / 28$ & 04:49:51.92 & -18.0590 & -176.9370 & 341 & 6. & $\mathrm{P}$ \\
\hline $2001 / 05 / 09$ & $17: 38: 26.06$ & -10.3660 & 161.2520 & 33 & 5.9 & $\mathrm{P}$ \\
\hline $2001 / 05 / 24$ & 18:07:57.0 & -38.836 & 175.210 & 261 & 5.5 & $\mathrm{P}, \mathrm{S}$ \\
\hline $2001 / 05 / 28$ & 08:37:06.25 & -6.6220 & 132.3260 & 49 & 5.9 & $\mathrm{P}$ \\
\hline $2001 / 06 / 03$ & $02: 41: 58.44$ & -29.6810 & -178.6150 & 188 & 6.7 & $\mathrm{P}$ \\
\hline $2001 / 06 / 05$ & 09:00:09.50 & -6.8840 & 146.3380 & 33 & 5.8 & $\mathrm{P}$ \\
\hline $2001 / 06 / 05$ & 15:13:58.25 & -6.7900 & 146.4210 & 10 & 5.9 & $\mathrm{P}$ \\
\hline $2001 / 06 / 23$ & 20:33:09.3 & -16.3030 & -73.5610 & 2 & 6.7 & $\mathrm{P}$ \\
\hline $2001 / 06 / 26$ & $04: 18: 32.7$ & -17.8310 & -71.6300 & 33 & 6.1 & $\mathrm{~S}$ \\
\hline
\end{tabular}

\section{D.2 Spectra}

Spectra are created from the single event phases used in the shear-wave splitting analysis and then stacked, to obtain an average spectrum characteristic for the phase. 


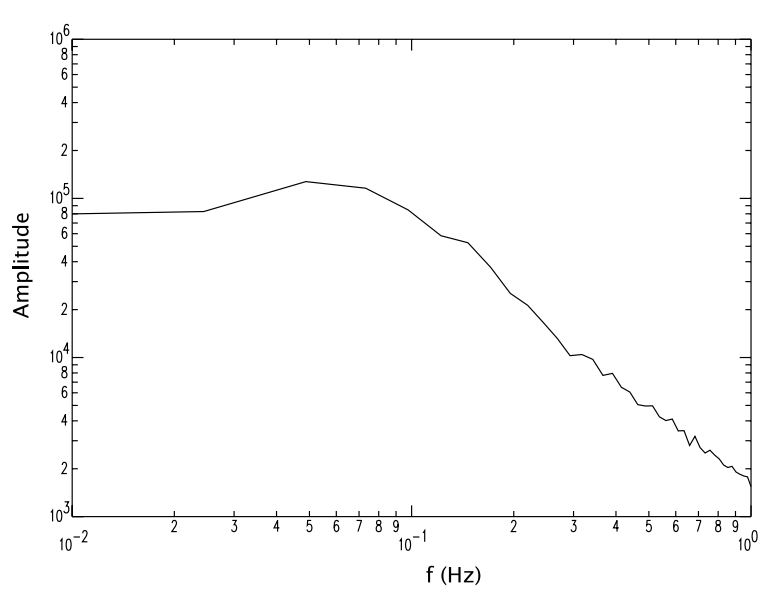

(a) Fast

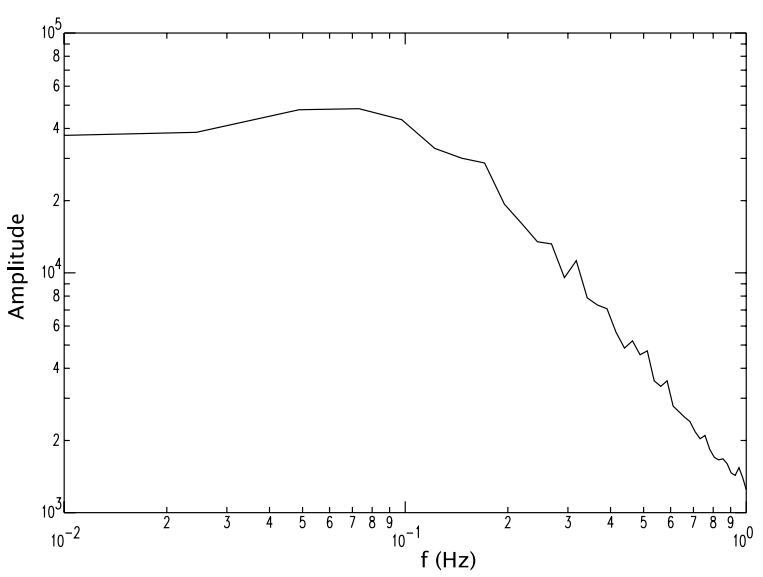

(b) Slow

FIGURE D.1 Average spectrum for SKS-phases

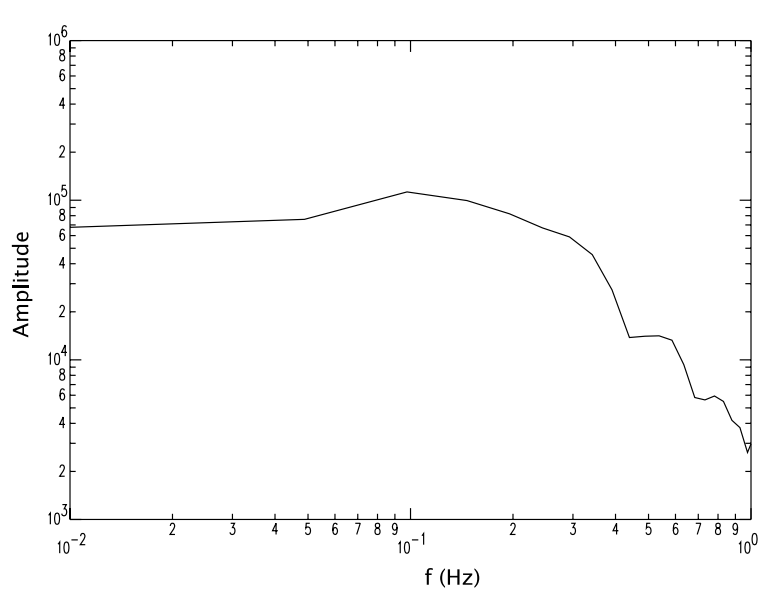

(a) Fast

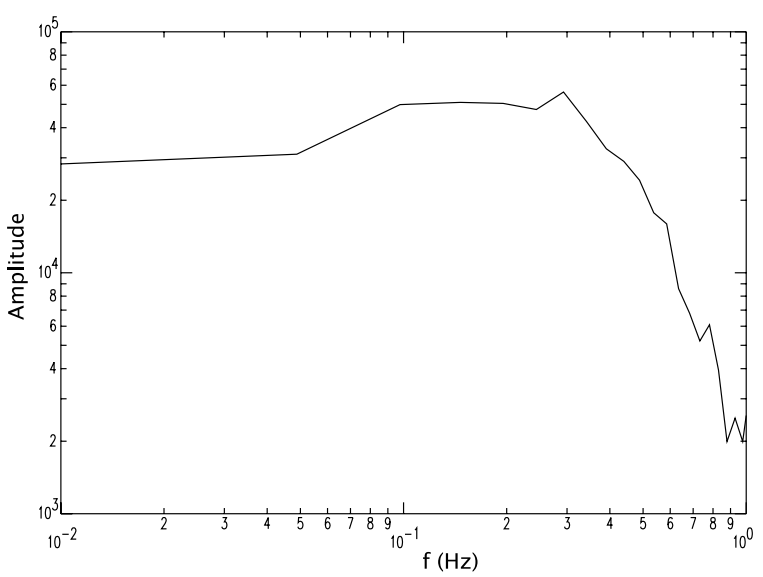

(b) Slow

FIGURE D.2 Average spectrum for S-phases

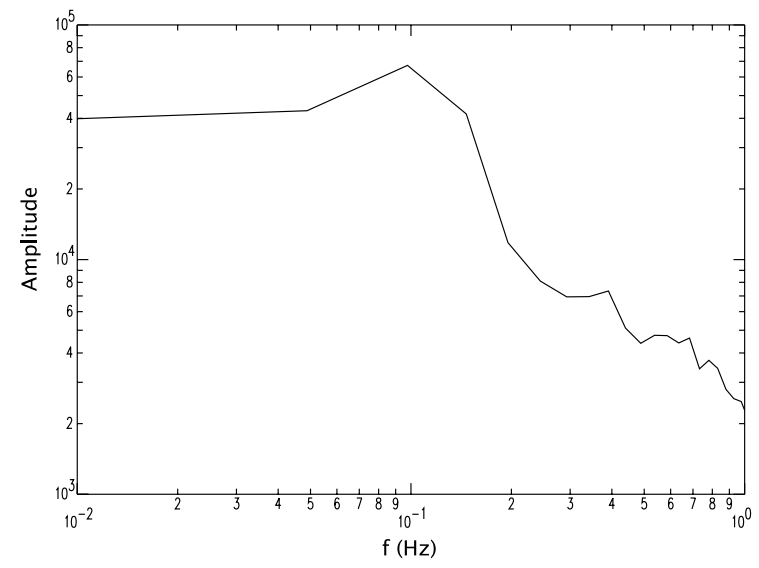

(a) Fast

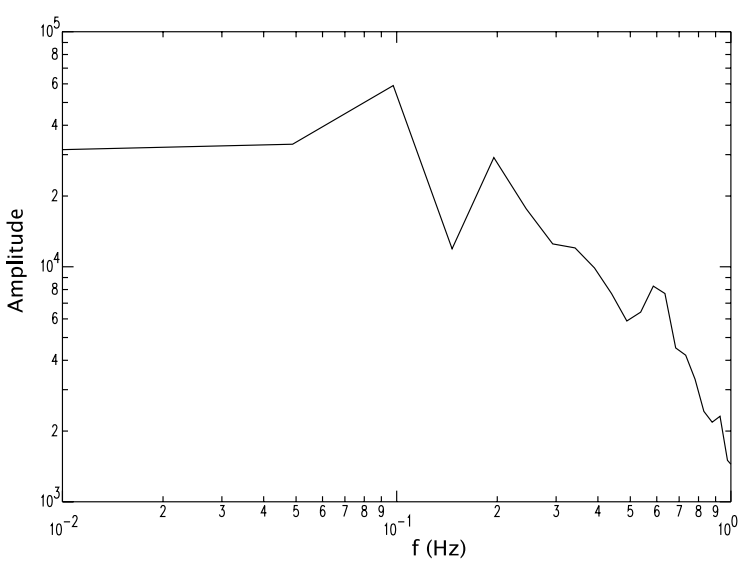

(b) Slow

FIGURE D.3 Average spectrum for ScS-phases. 


\section{APPENDIX E}

\section{Shear-wave splitting results}

\section{E.1 Results from automated splitting code}

The following table lists the single shear-wave splitting results discussed in chapter 5 . 


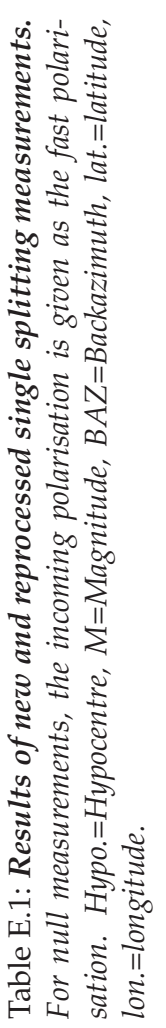

\begin{tabular}{|c|c|c|c|c|c|c|c|c|c|c|c|c|c|c|c|c|c|c|c|c|c|c|}
\hline $\begin{array}{l}\text { 节 } \\
\text { ă }\end{array}$ & & & & & & & $b$ & & & & & & & & & $\infty$ & $|<| \ll$ & $\infty$ & $\ll \mid \oplus$ & & & \\
\hline & 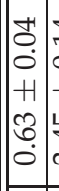 & 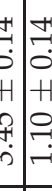 & & $\begin{array}{l}0 \\
+1 \\
+1 \\
-1\end{array}$ & $\left|\begin{array}{l}0 \\
+1 \\
0 \\
0 \\
\hdashline \\
-1\end{array}\right|$ & & 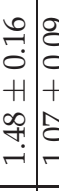 & 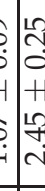 & $\overline{\vec{z}}$ & & & & & $\overline{\vec{z}}$ & $=\mid \begin{array}{l}2 \\
\frac{3}{3} \\
0 \\
+1 \\
2 \\
\infty \\
\infty \\
0\end{array}$ & $\begin{array}{l}\hat{f} \\
0 \\
+ \\
+ \\
\hat{0} \\
\text { ì } \\
\end{array}$ & 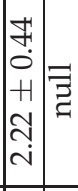 & $\begin{array}{l}0 \\
0 \\
0 \\
+1 \\
0 \\
0 \\
\dot{-i} \\
\end{array}$ & 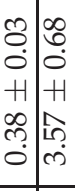 & $\overline{\vec{z}}$ & 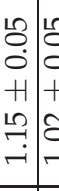 & 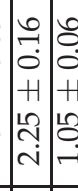 \\
\hline & $\mid \begin{array}{c}0 \\
\dot{m} \\
+1 \\
0 \\
0 \\
\infty \\
\infty \\
\infty\end{array}$ & 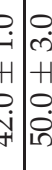 & & 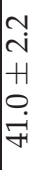 & & & 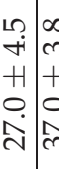 & 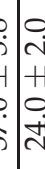 & & & & & & 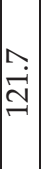 & 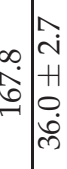 & $\mid \begin{array}{c}\Lambda \\
\infty \\
+1 \\
0 \\
\vec{\lambda}\end{array}$ & 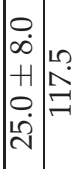 & 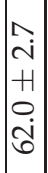 & 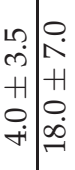 & & 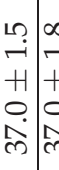 & 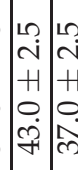 \\
\hline 边 & 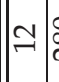 & & & & & & $\bowtie \curvearrowright$ & $\mid \stackrel{\infty}{I}$ & & & $m$ & & & $\mathbb{Z}$ & \begin{tabular}{l}
0 \\
\hdashline \\
$⿱ 亠 乂$
\end{tabular} & $\infty$ & $\infty \stackrel{\infty}{=}$ & $\infty$ & $\infty$ & $\stackrel{\infty}{=}$ & $m \rightleftharpoons$ & \\
\hline 思 & $\tilde{\omega}$ & w & & & & $c$ & $\begin{array}{c}w \\
v \\
v\end{array}$ & 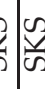 & w & & 㟧 & & & & & $\frac{s}{w}$ & 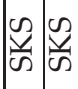 & $\left|\begin{array}{l}\tilde{U} \\
\tilde{w}\end{array}\right|$ & 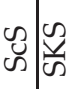 & $\frac{\infty}{\infty}$ & $\begin{array}{c}\tilde{y} \\
\tilde{w} \\
\tilde{c}\end{array}$ & or \\
\hline$\Sigma$ & $\mid \begin{array}{lll}7 \\
0\end{array}$ & $\begin{array}{l}0 \\
0\end{array}$ & & & $\left|\begin{array}{l}\infty \\
\dot{1} \\
\dot{1}\end{array}\right|$ & 6 & 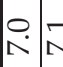 & 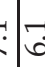 & 6 & t: & $\widehat{\sigma}$ & $\dot{b}_{0}^{0}$ & 16 & G. & ن. & $\mid \begin{array}{c}0 \\
0 \\
0\end{array}$ & $\left(\begin{array}{c}m \\
\hdashline\end{array} \mid\right.$ & |ֶ. & 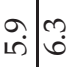 & 7 & \begin{tabular}{l|l}
0 \\
\end{tabular} & \\
\hline $\bar{\nu}^{2}$ & $\overbrace{}^{\circ}$ & & is & & & & $\stackrel{1}{\infty}$ & 4 & $\mp$ & & & & & 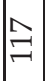 & 이젯 & |゙ & Jু & 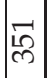 & 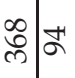 & dָ & 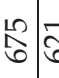 & مَ \\
\hline & 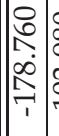 & & de & & & cُ & 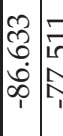 & $\begin{array}{l}0 \\
0 \\
0 \\
0 \\
0\end{array}$ & $\begin{array}{l}\text { فे } \\
\text { فे }\end{array}$ & & 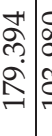 & & & $\mid \begin{array}{l}\infty \\
0 \\
0 \\
0 \\
0 \\
1\end{array}$ & 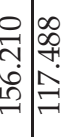 & $\left|\begin{array}{c}\stackrel{2}{\hat{N}} \\
\infty \\
\infty \\
1\end{array}\right|$ & 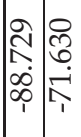 & 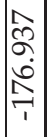 & 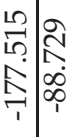 & 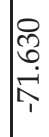 & 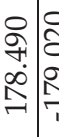 & 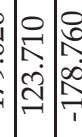 \\
\hline & $\left.\mid \begin{array}{c}0 \\
0 \\
0 \\
\hdashline \\
\hdashline \\
1\end{array}\right)$ & & & & & & 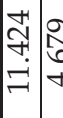 & 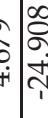 & హ. & & & & & & 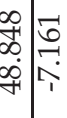 & $\mid \begin{array}{l}\hat{\alpha} \\
\hat{\alpha} \\
\dot{\epsilon}\end{array}$ & 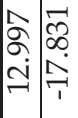 & $\mid \begin{array}{c}1 \\
8 \\
0 \\
0 \\
? \\
1\end{array}$ & 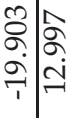 & 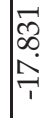 & 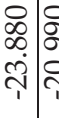 & $\begin{array}{lll}8 & 0 \\
8 & 0 \\
0 & 0 \\
0 & 7\end{array}$ \\
\hline 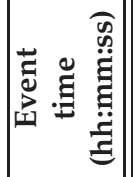 & 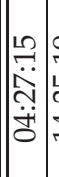 & & & & & $\ddot{g}$ & 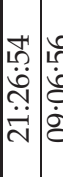 & & 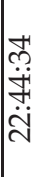 & & $\begin{array}{l}0 \\
0 \\
\end{array}$ & & & & 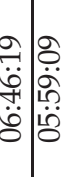 & 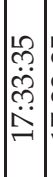 & 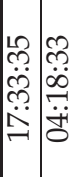 & 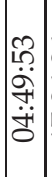 & 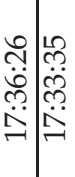 & $\underset{\infty}{\infty}$ & 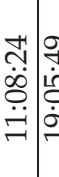 & 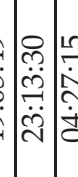 \\
\hline 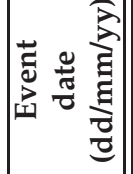 & 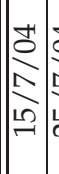 & $\sqrt{3}$ & & $\pi$ & & & 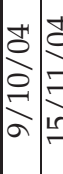 & $=\frac{1}{2}$ & ल & & & & & & 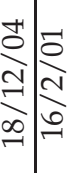 & $\begin{array}{l}- \\
0 \\
- \\
m \\
-\end{array} \mid$ & 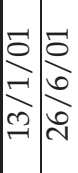 & $\begin{array}{c}5 \\
\vdots \\
+ \\
\infty \\
\text { సి }\end{array}$ & 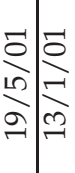 & & 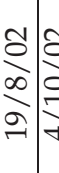 & 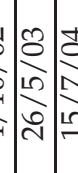 \\
\hline 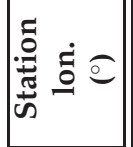 & 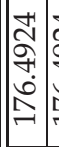 & 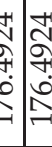 & & & & & 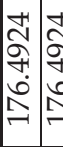 & 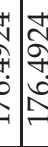 & $\tilde{\sigma}$ & & 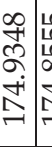 & & & 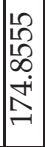 & 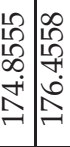 & 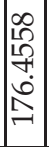 & 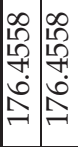 & 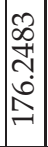 & 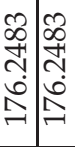 & 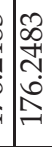 & 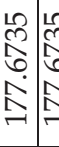 & 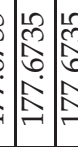 \\
\hline 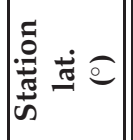 & 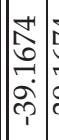 & 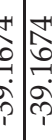 & & & & & 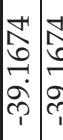 & 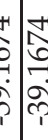 & & & 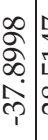 & & & $\begin{array}{l}\hat{y} \\
\text { in } \\
\infty \\
\infty\end{array}$ & 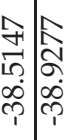 & 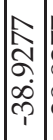 & 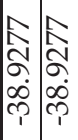 & 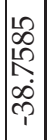 & 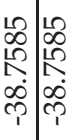 & $\left\{\begin{array}{l}2 \\
\infty \\
2 \\
\infty \\
\infty \\
0 \\
p\end{array}\right.$ & 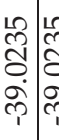 & 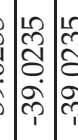 \\
\hline 宽 & 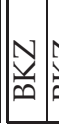 & 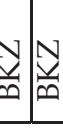 & & & & & & & & & & & & & & & 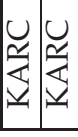 & $z$ & בע & $\approx$ & $\begin{array}{l}N \\
Z \\
Z\end{array}$ & $\frac{1}{2}$ \\
\hline
\end{tabular}




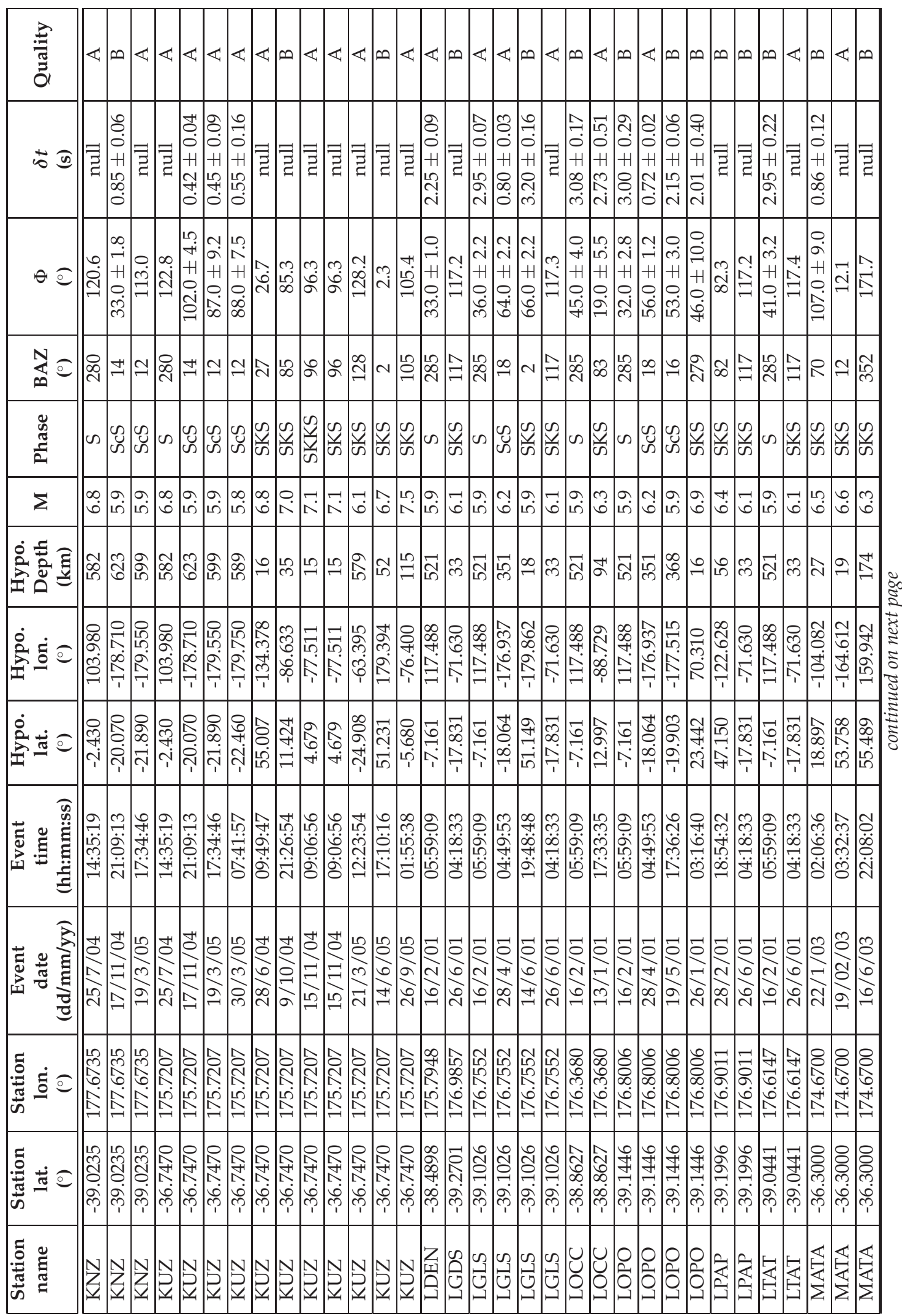




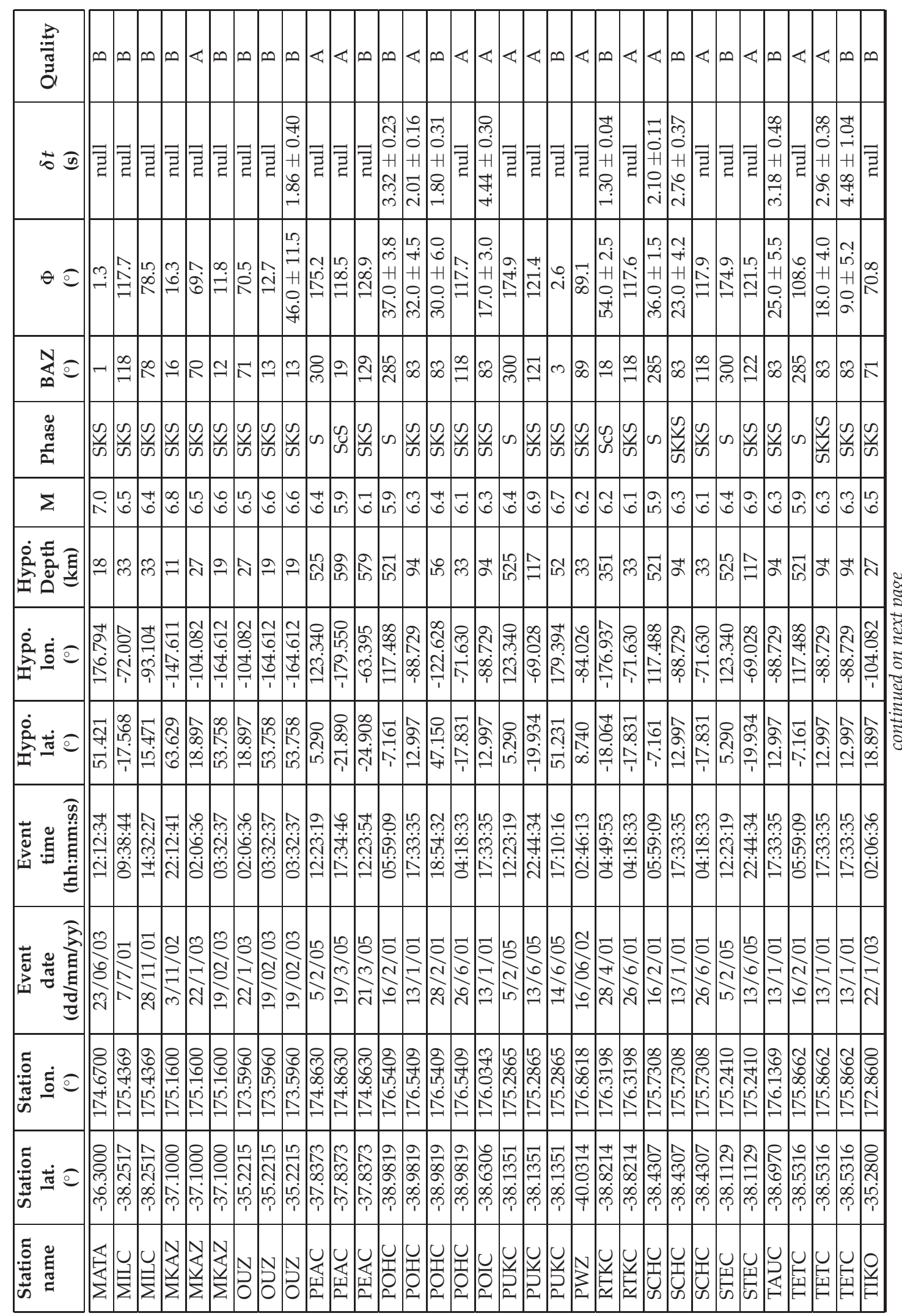




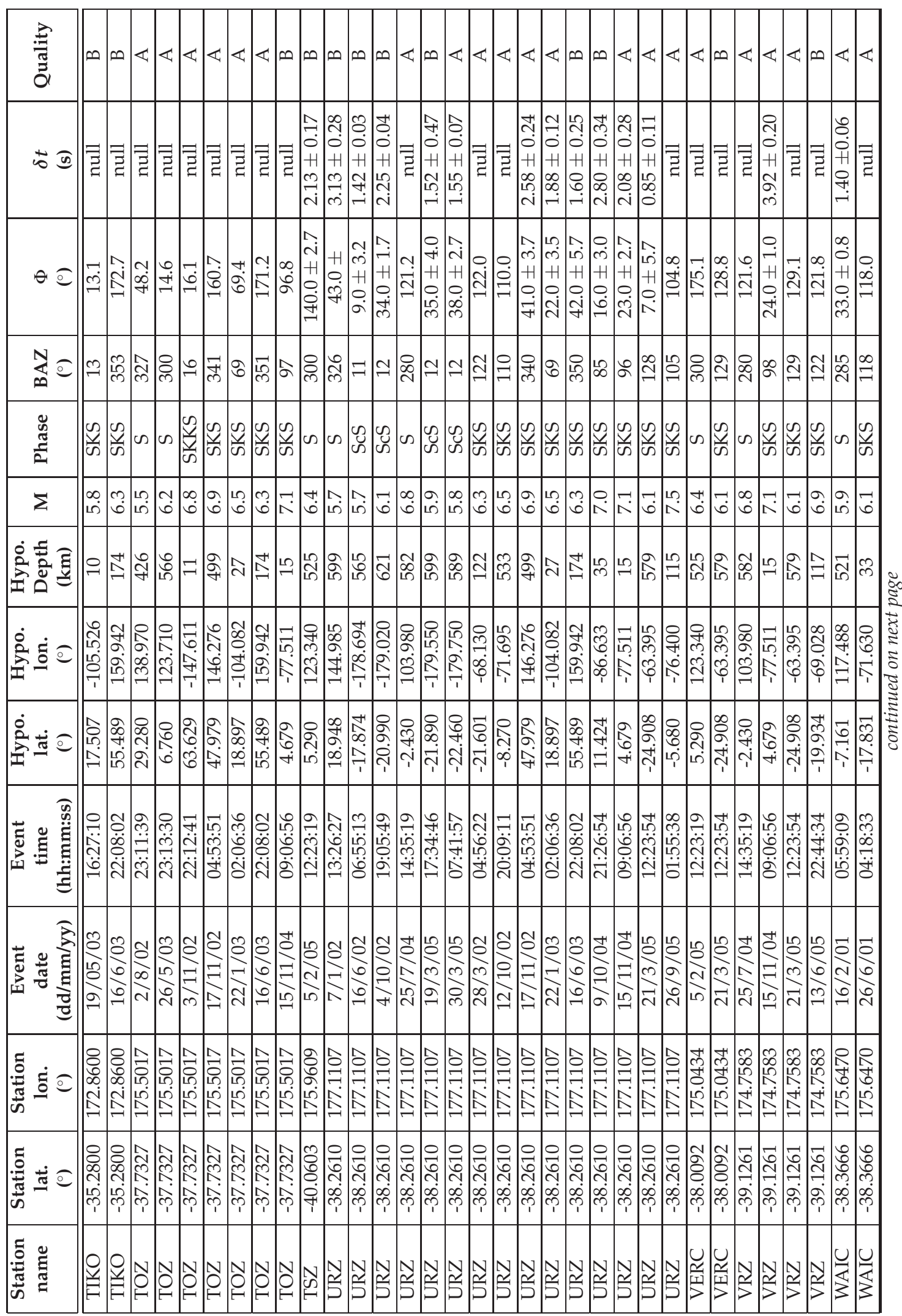




\begin{tabular}{|c|c|c|c|c|c|c|c|c|c|c|c|c|c|c|c|c|c|c|c|c|c|}
\hline 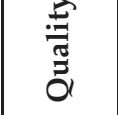 & & $\infty$ & & $\varangle$ & & & & $\infty$ & & & $b$ & & ص. & $\ll$ & & & $\varangle$ & & ת & & $\ll$ \\
\hline$\vec{\infty} \widehat{\infty}$ & 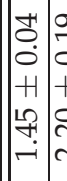 & 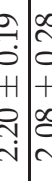 & & 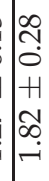 & $\vec{z}$ & $\overline{\vec{z}}$ & 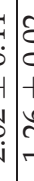 & & & 就 & & & $\overrightarrow{\bar{z}}$ & $\overline{\bar{\Xi}}$ & & & $\overline{\vec{z}}$ & & 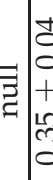 & & $\overrightarrow{\vec{z}}$ \\
\hline$\theta \subsetneq$ & 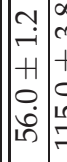 & & & 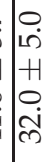 & & & 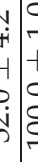 & & & & 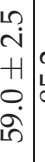 & & & \begin{tabular}{l}
0 \\
0 \\
$\infty$ \\
\hdashline \\
\hdashline
\end{tabular} & & & 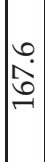 & & 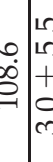 & & 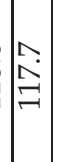 \\
\hline$\sum_{\infty}^{N} 0$ & 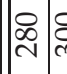 & हి & & $2 \infty$ & ते & 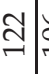 & $\stackrel{2}{2}$ & f & 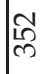 & In & $\vec{c}$ & & 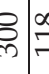 & స్ & & & 亦 & & הిר & & $\stackrel{\infty}{=}$ \\
\hline 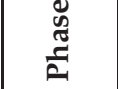 & & is & & $\frac{\omega}{\omega}$ & 娄 & w) & d & $\mid \begin{array}{l}\frac{4}{5} \\
\frac{5}{4}\end{array}$ & & e & s) & & is & $\tilde{w}$ & 8 & & $\frac{\omega}{\omega}$ & $\frac{n}{n}$ & is & & $\frac{w}{w}$ \\
\hline$\Sigma$ & $\left|\begin{array}{l}\infty \\
0 \\
0\end{array}\right|$ & t: & & 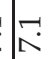 & $\mid-10$ & ब̧. & 5 & ç & مె & ف. & 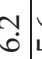 & & $\begin{array}{r}\mid \\
\\
\end{array}$ & 6 & : & & o. & & r. & & 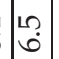 \\
\hline 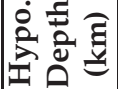 & 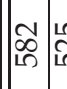 & है & & $\stackrel{10}{\sim}$ & & $\exists$ & 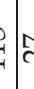 & $\sigma$ & $\stackrel{I}{I}$ & & 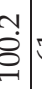 & & & & & & 우 & & הై & & $\infty$ \\
\hline 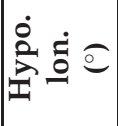 & 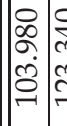 & 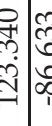 & & 离 & $\mid \begin{array}{l}2 \\
0 \\
0 \\
0 \\
0 \\
1 \\
\end{array}$ & 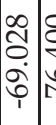 & : & t. & 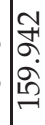 & o & 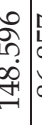 & & 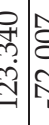 & 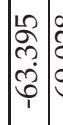 & in & & 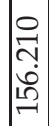 & Li⿱ & 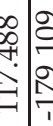 & & $\mid \begin{array}{l}\hat{O} \\
\mathbf{8} \\
\mathrm{i} \\
\hat{N}\end{array}$ \\
\hline 递灾 & 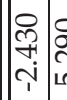 & & & 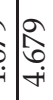 & & 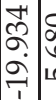 & $\mid \begin{array}{l}1 \\
0 \\
0 \\
0 \\
0\end{array}$ & is & 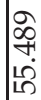 & గొ & 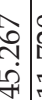 & & 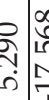 & 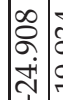 & th & & \begin{tabular}{l}
$\infty$ \\
$\infty$ \\
$\infty$ \\
$\infty$ \\
$\infty$ \\
$\infty$ \\
\hdashline
\end{tabular} & $f$ & 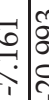 & & 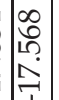 \\
\hline 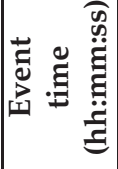 & 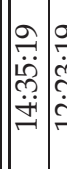 & & & 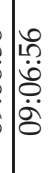 & . & & غ & $\begin{array}{l}\hat{\alpha} \\
\dot{\hat{\alpha}} \\
\dot{\varepsilon}\end{array}$ & $\begin{array}{l}\tilde{\alpha} \\
\dot{d} \\
\ddot{d} \\
\ddot{d}\end{array}$ & & 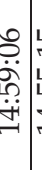 & & 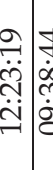 & 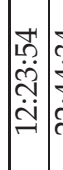 & & & 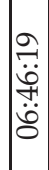 & & 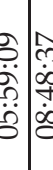 & & 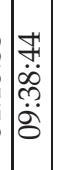 \\
\hline 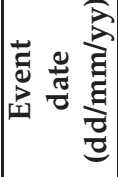 & 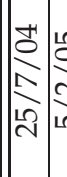 & 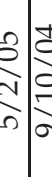 & & $\begin{array}{l}2 \\
2\end{array}$ & $\mid \begin{array}{l}0 \\
\infty \\
2 \\
\\
\end{array}$ & 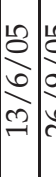 & & 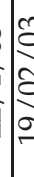 & $\frac{\infty}{2}$ & & กำ & & 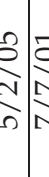 & 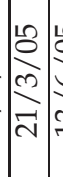 & & & 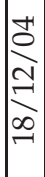 & 10 & 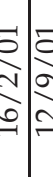 & & 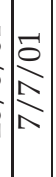 \\
\hline 莞 & 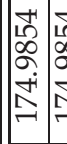 & | & & 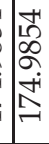 & 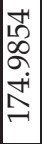 & 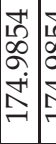 & $\sum^{g}$ & $g$ & 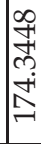 & 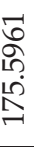 & . & & 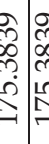 & 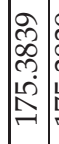 & & & 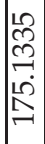 & $m$ & 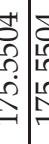 & & 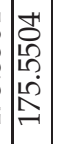 \\
\hline 总 & 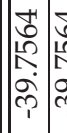 & 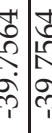 & & 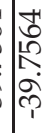 & 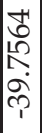 & के & & & 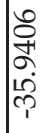 & & 6 & & 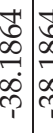 & 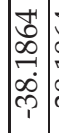 & & & $\mid \begin{array}{l}0 \\
0 \\
0 \\
0 \\
0 \\
0 \\
1\end{array}$ & & 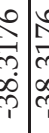 & & 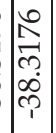 \\
\hline 营 & $\begin{array}{ll}2 & 1 \\
3 & 3 \\
3 & 3\end{array}$ & 3 & & & 3 & & & & & 3 & 3 & & 3 & 3 & & & 㓐 & & 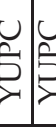 & & 就 \\
\hline
\end{tabular}




\section{E.2 Shear-wave splitting analysis using SplitLab}

During the course of this study, a new code to perform shear-wave splitting measurements called SplitLab [Wüstefeld et al., 2008] was in development and tested on North Island data. The code is Matlab ${ }^{\mathbb{R}}$ based with an intuitive graphical interface. The code delivers shearwave splitting parameters derived with two different shear-wave splitting methods, the method by Silver \& Chan [1991], as used in the main part of this study (cf. chapter 5) and a cross-correlation method [Levin et al., 1999]. Comparison of the two splitting parameter pairs provides the advantage of easier identification of stable results and null measurements [Wüstefeld \& Bokelmann, 2007].

The SplitLab code is now robust and published [Wüstefeld et al., 2008]. It is a useful tool to get a good handle on shear-wave splitting analysis and for quality assessment. However, the process cannot be automated and identification of the best-fit window depends on the subjectivity of the analyst.

For this study, comparison of the automated results (table E.1) with the SplitLab results (see table below) gives further proof of the stability of the analysis. 

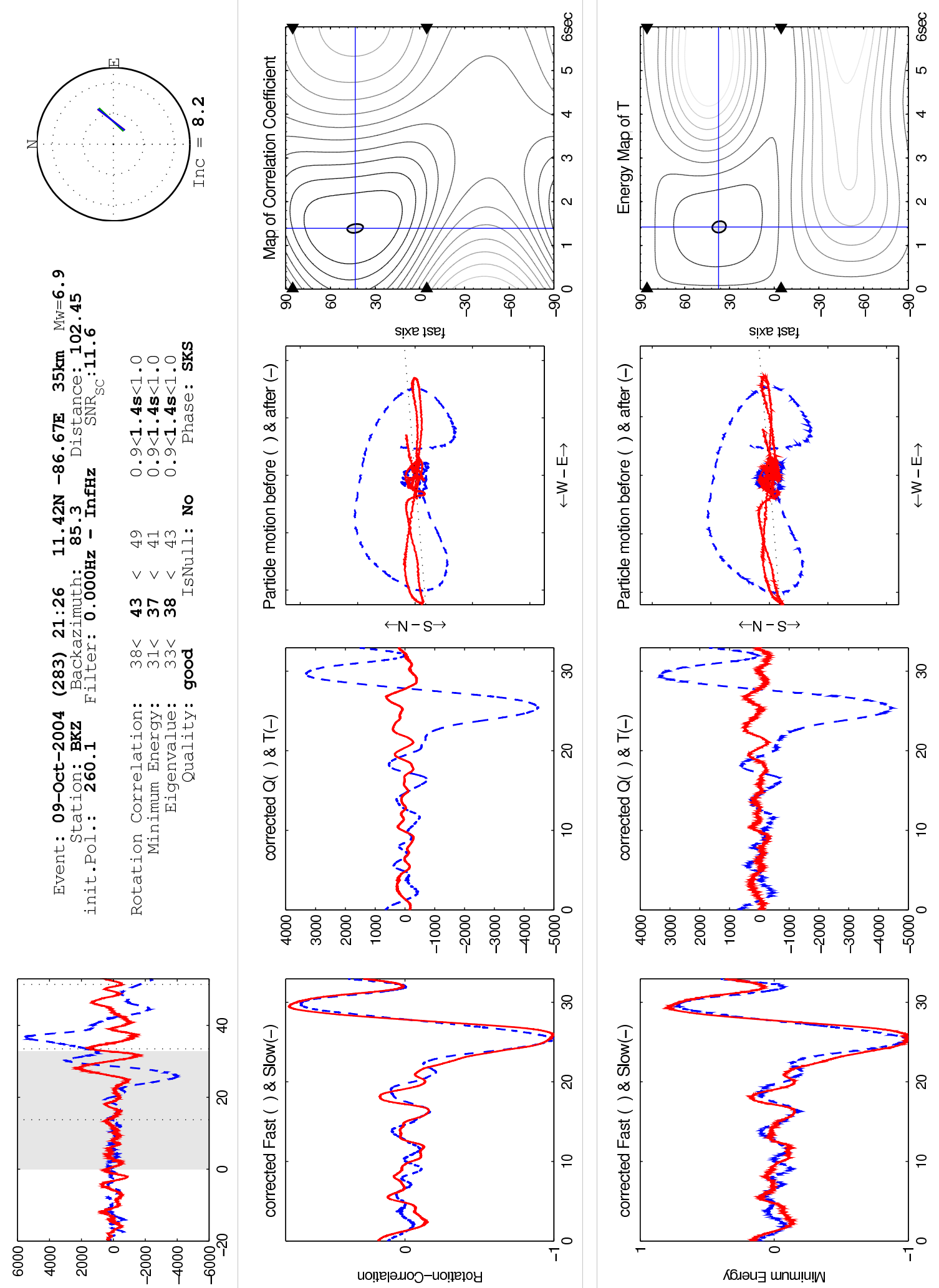

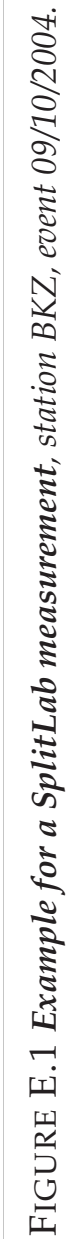
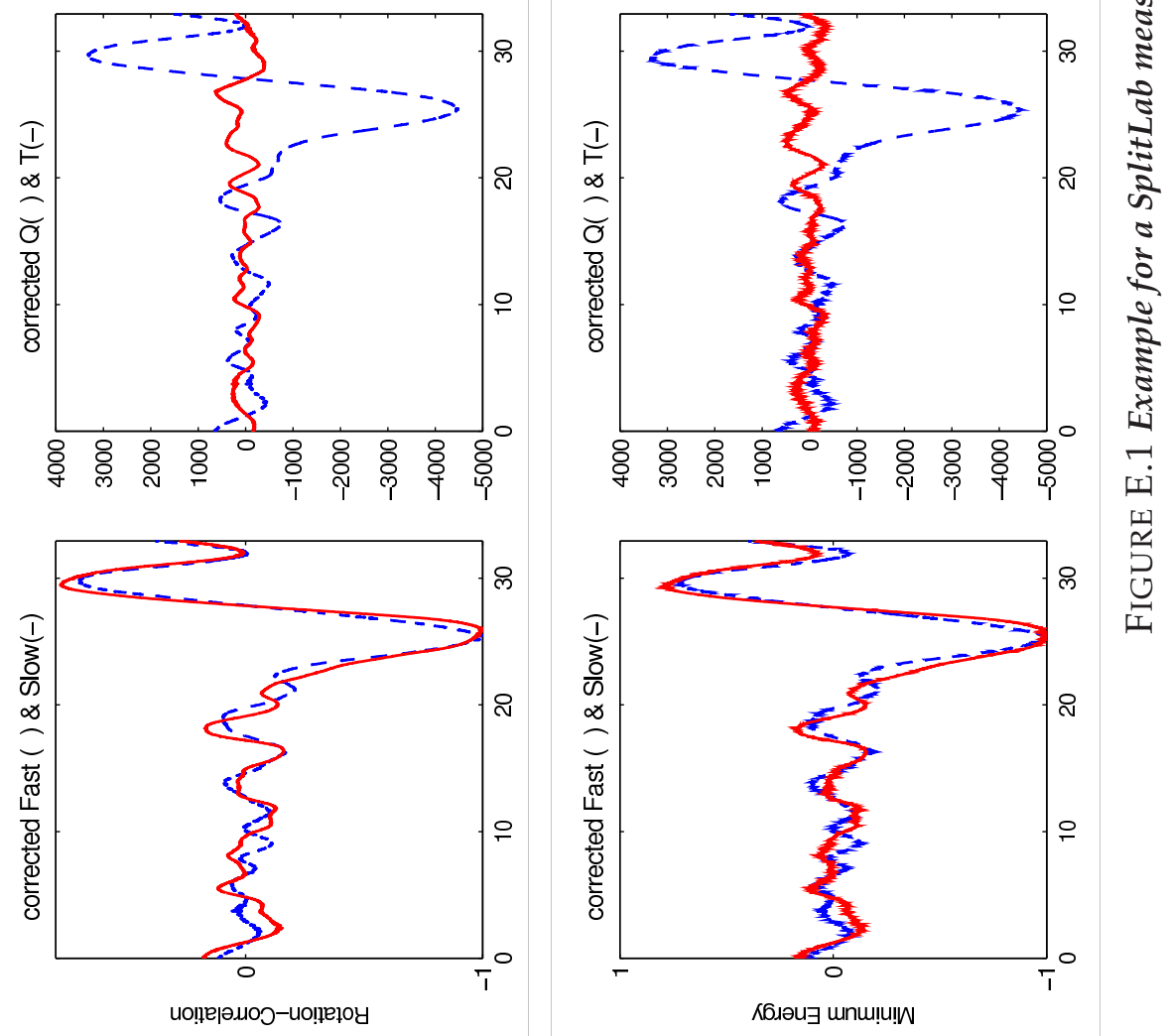


\begin{tabular}{|c|c|c|}
\hline & $\bar{z}$ & 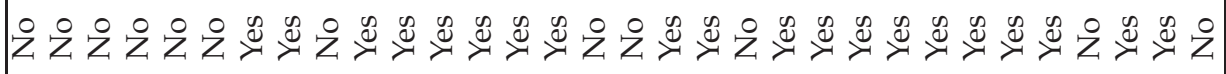 \\
\hline & $\frac{3}{\frac{3}{\pi}}$ & 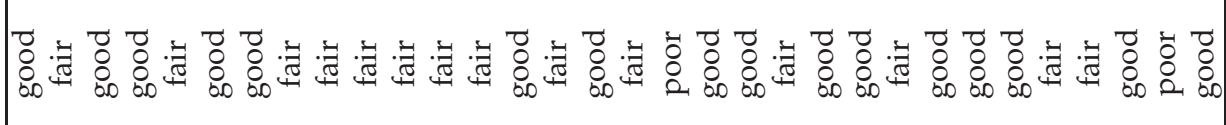 \\
\hline & $\sum_{i}^{\infty} \widehat{\omega}$ & 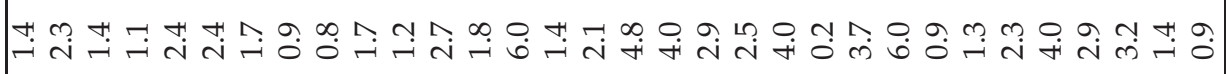 \\
\hline & $\theta$ & 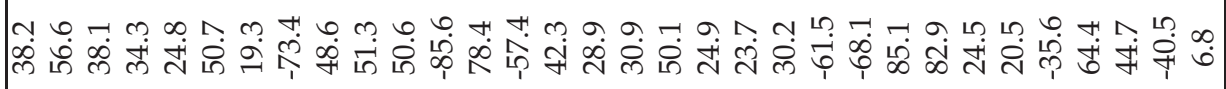 \\
\hline 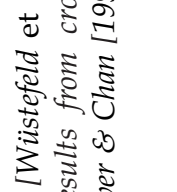 & $\underbrace{\infty}_{\infty} \infty$ & 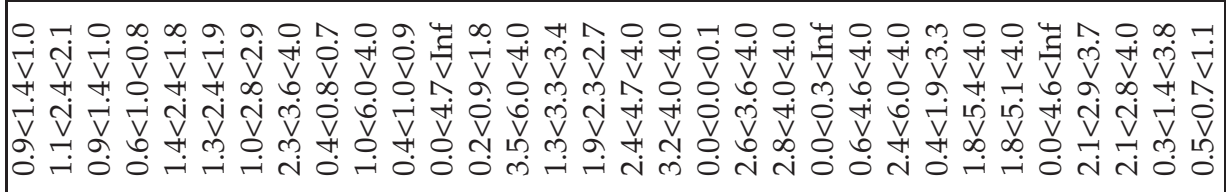 \\
\hline 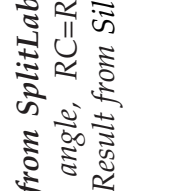 & & 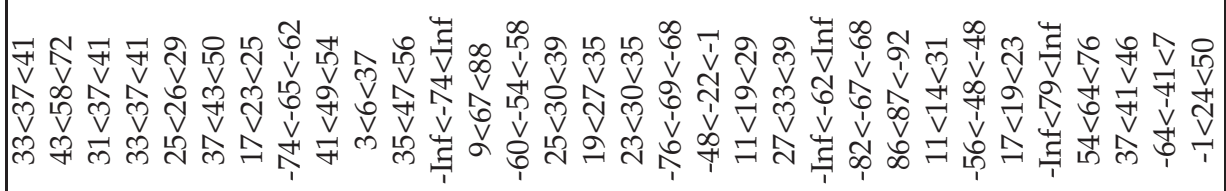 \\
\hline & 苛 & 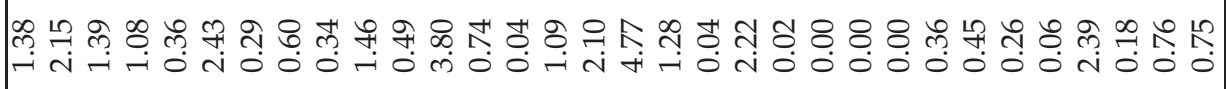 \\
\hline$\sum$ घ & $\ddot{\ddot{\theta}} \tilde{\theta}$ & 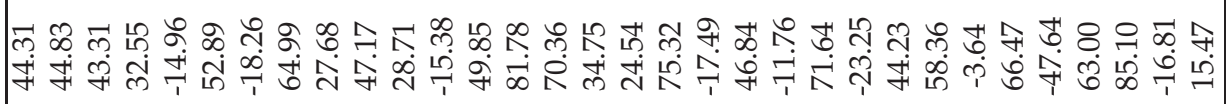 \\
\hline 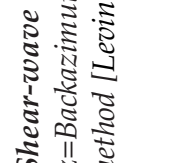 & 辛 & 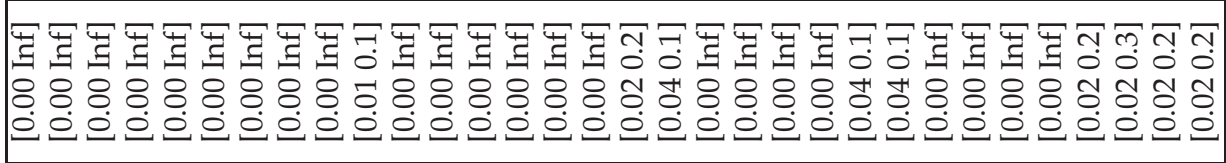 \\
\hline.$\overline{\bar{c}}$ & $\dot{\dot{g}} \sigma$ & 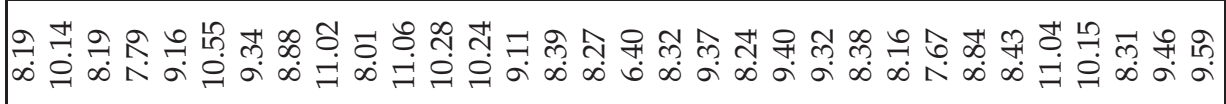 \\
\hline & 跎 & 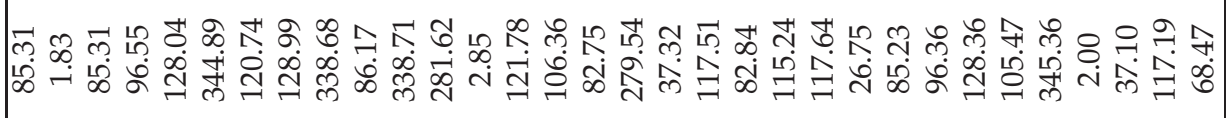 \\
\hline & 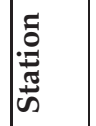 & 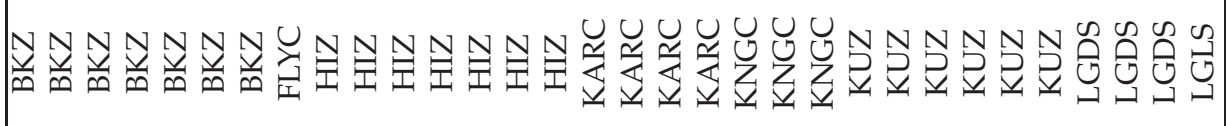 \\
\hline & 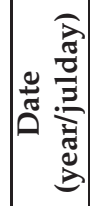 & 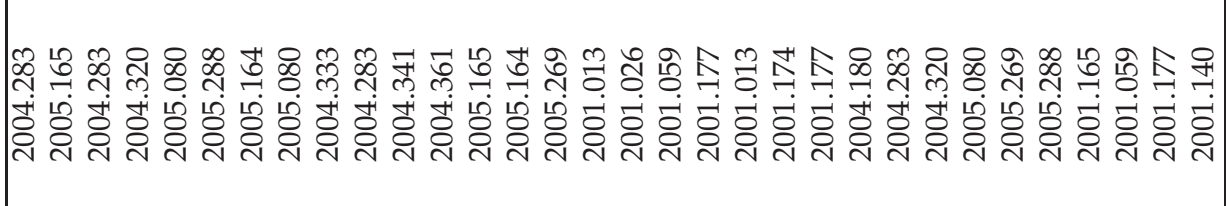 \\
\hline
\end{tabular}




\begin{tabular}{|c|c|}
\hline 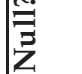 & 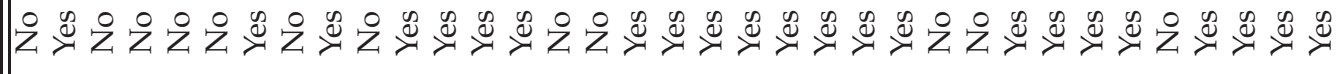 \\
\hline$\sum_{\substack{2 \\
\frac{\pi}{2}}}^{2}$ & 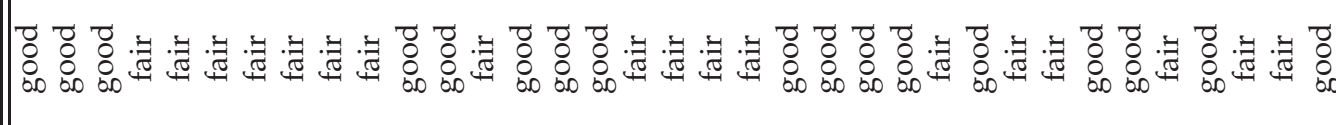 \\
\hline $\mathbb{1}_{i=}^{\infty}$ & 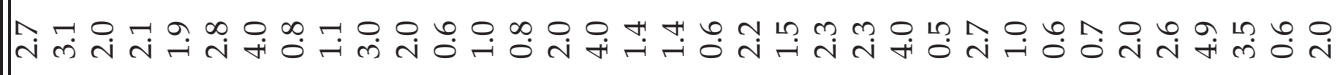 \\
\hline$\vec{\omega}$ & 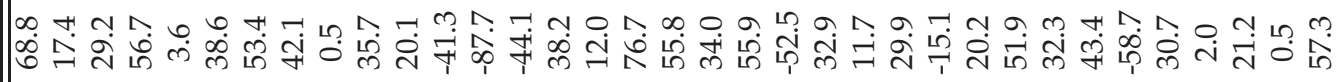 \\
\hline$\underbrace{\infty}_{\infty}$ & 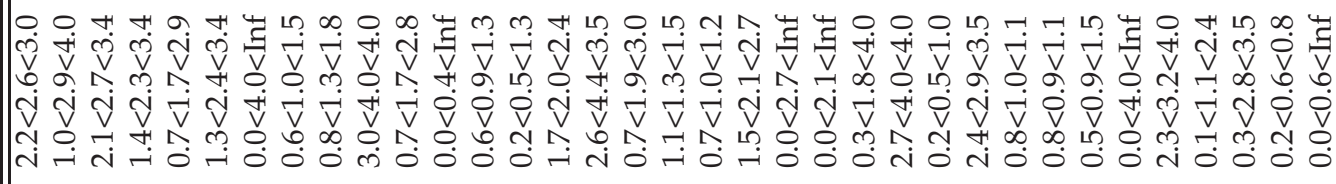 \\
\hline & 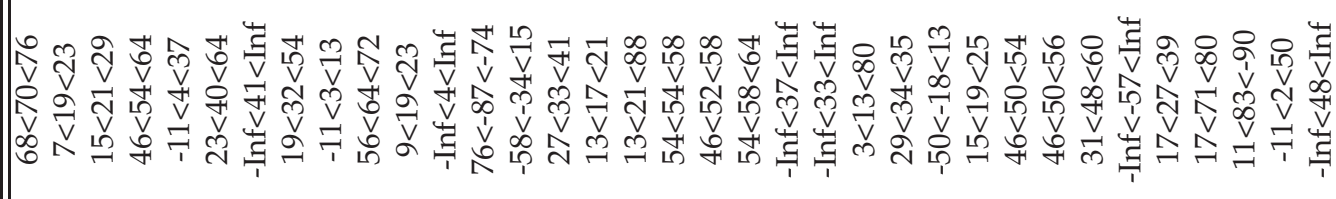 \\
\hline$\approx$ & 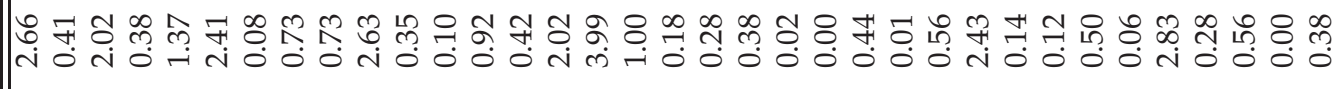 \\
\hline$\ddot{\theta}$ & 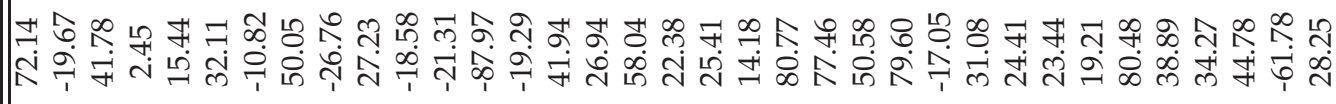 \\
\hline 宔 & 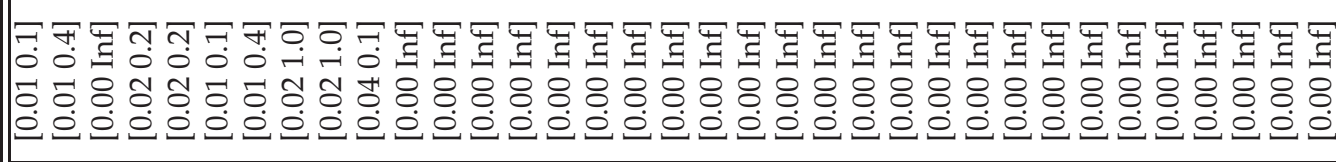 \\
\hline$\dot{\Xi} \sigma$ & 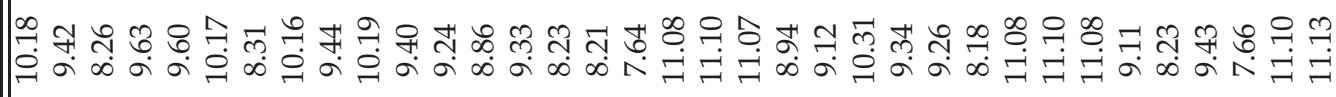 \\
\hline$\stackrel{N}{0} 0$ & 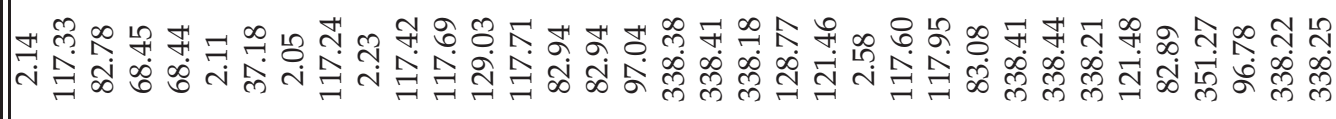 \\
\hline 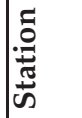 & 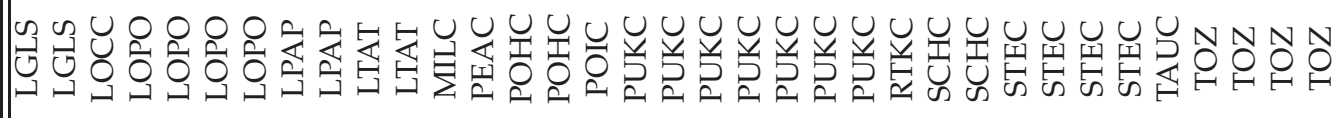 \\
\hline 宽 & 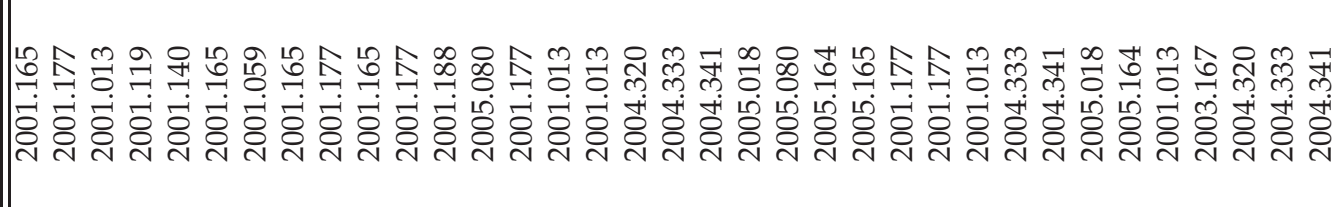 \\
\hline
\end{tabular}




\begin{tabular}{|c|c|}
\hline 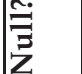 & 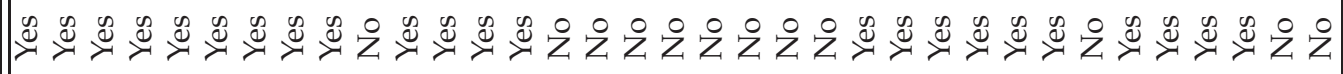 \\
\hline 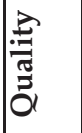 & 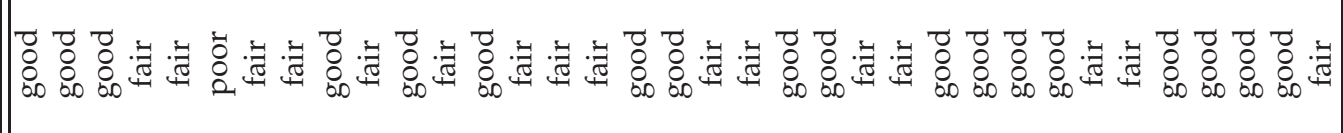 \\
\hline$\vec{i}_{i}^{\infty} \widehat{\infty}$ & 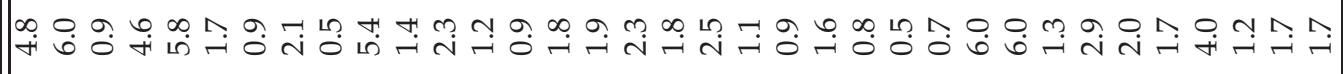 \\
\hline 岕。 & 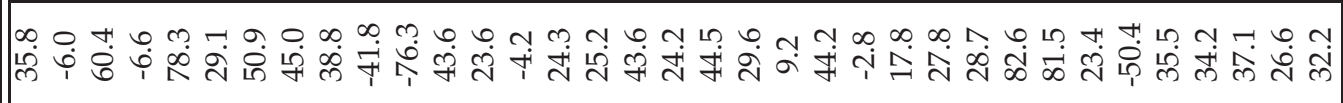 \\
\hline 㭊 & 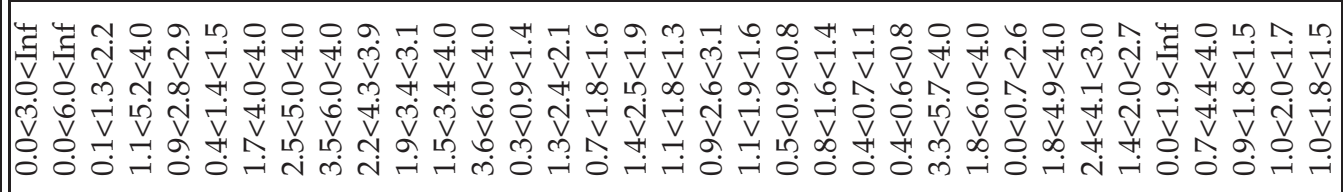 \\
\hline$\ddot{\omega} \sigma$ & 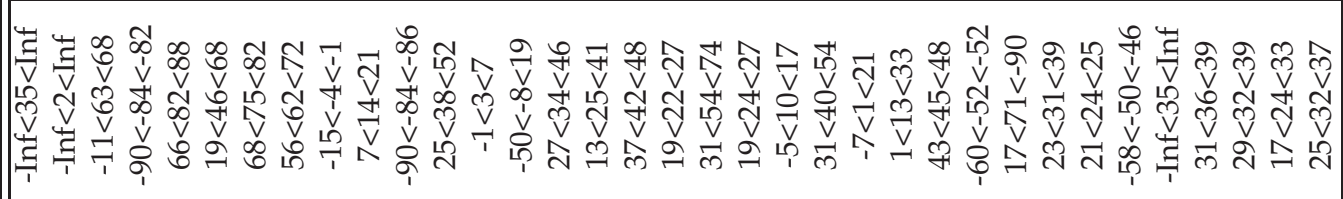 \\
\hline$\approx$ & 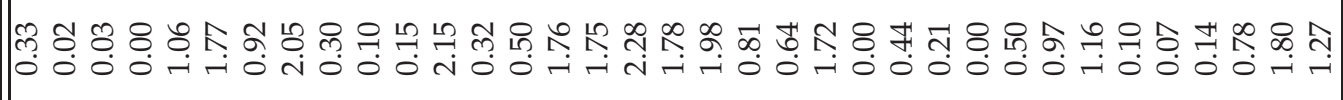 \\
\hline$\ddot{\theta} \sigma$ & 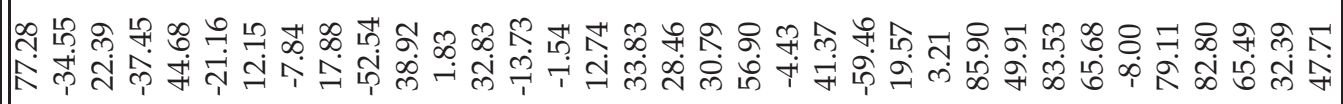 \\
\hline 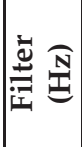 & 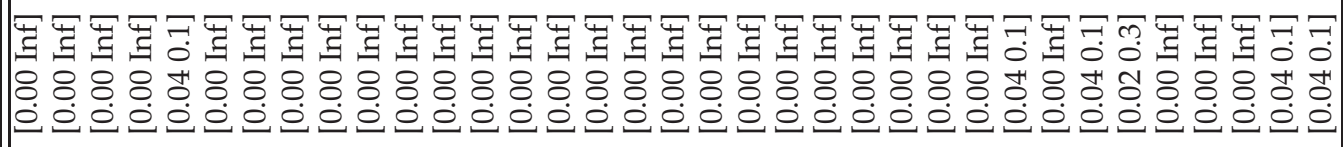 \\
\hline$\dot{\ddot{g}} \precsim$ & 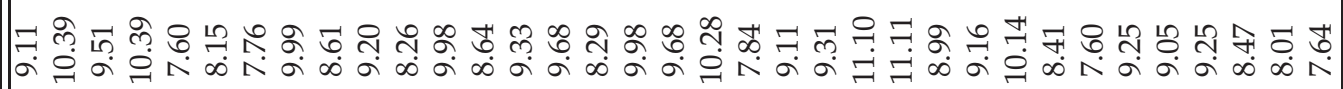 \\
\hline 赵 $\widetilde{C}$ & 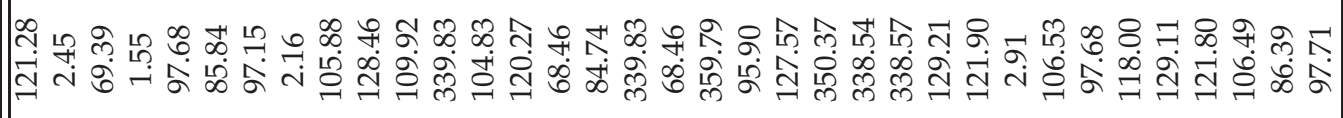 \\
\hline 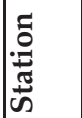 & 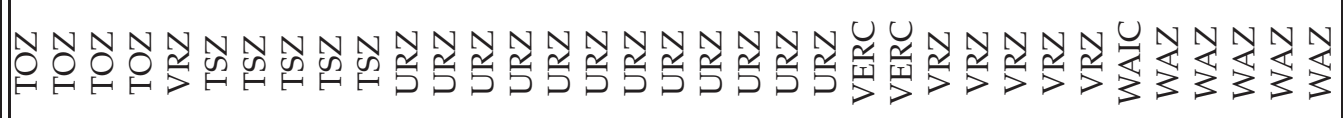 \\
\hline 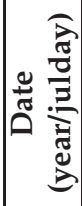 & 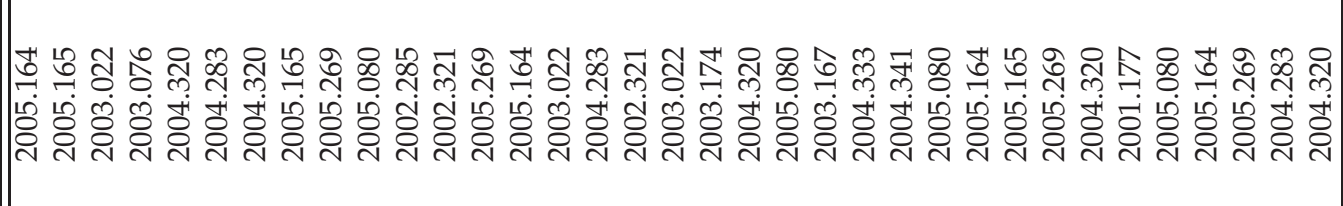 \\
\hline
\end{tabular}




\begin{tabular}{|c|c|}
\hline$\hat{\bar{\Xi}}$ & 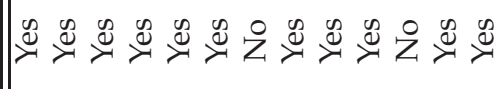 \\
\hline 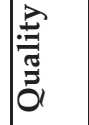 & 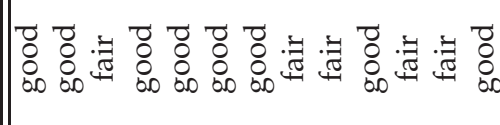 \\
\hline$\frac{1}{10}$ & 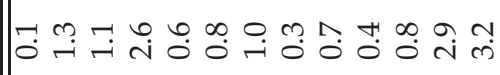 \\
\hline 离 & 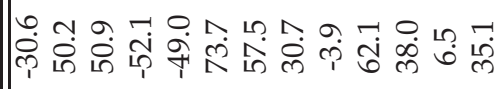 \\
\hline 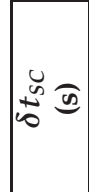 & 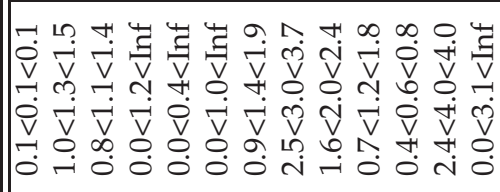 \\
\hline & 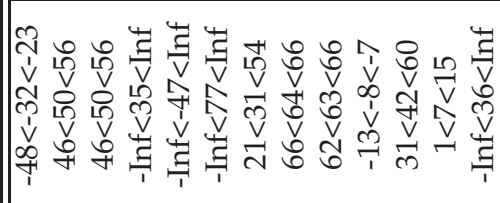 \\
\hline$\underset{\pi}{\approx} \widehat{\infty}$ & 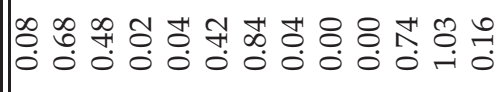 \\
\hline & 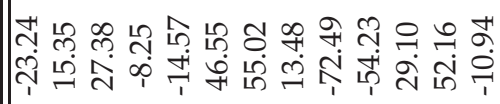 \\
\hline 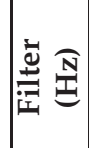 & 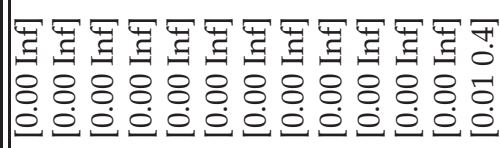 \\
\hline$\dot{g} \precsim$ & 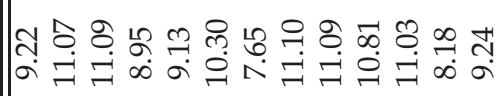 \\
\hline స్ & 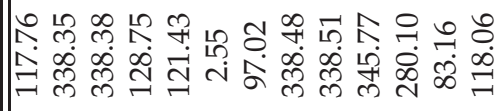 \\
\hline . & 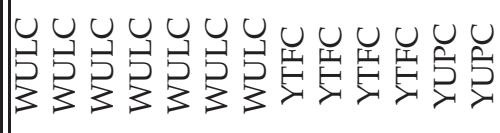 \\
\hline 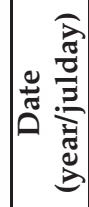 & 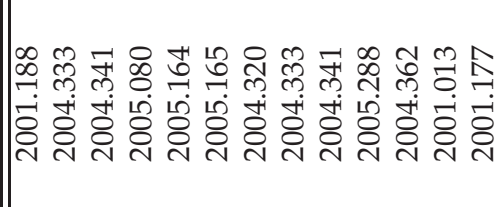 \\
\hline
\end{tabular}




\section{APPENDIX F}

\section{Analytical small-scale modelling}

\section{F.1 Tandon and Weng theory - Terms}

The following terms are used in the calculations:

$$
\begin{aligned}
A & =2 B_{2} B_{3}-B_{1}\left(B_{4}+B_{5}\right), \\
A_{1} & =D_{1}\left(B_{4}+B_{5}\right)-2 B_{2}, \\
A_{2} & =\left(1+D_{1}\right) B_{2}-\left(B_{4}+B_{5}\right), \\
A_{3} & =B_{1}-D_{1} B_{3}, \\
A_{4} & =\left(1+D_{1}\right) B_{1}-2 B_{3}, \\
A_{5} & =\left(1-D_{1}\right) /\left(B_{4}-B_{5}\right)
\end{aligned}
$$

$$
\begin{aligned}
& B_{1}=c D_{1}+D_{2}+(1-c)\left(D_{1} S_{1111}+2 S_{2211}\right), \\
& B_{2}=c+D_{3}+(1-c)\left(D_{1} S_{1122}+S_{2222}+S_{2233}\right), \\
& B_{3}=c+D_{3}+(1-c)\left[S_{1111}+\left(1+D_{1}\right) S_{2211}\right], \\
& B_{4}=c D_{1}+D_{2}+(1-c)\left(S_{1122}+D_{1} S_{2222}+S_{2233}\right), \\
& B_{5}=c+D_{3}+(1-c)\left(S_{1122}+S_{2222}+D_{1} S_{2233}\right)
\end{aligned}
$$

$$
\begin{aligned}
& D_{1}=1+2\left(\mu_{1}-\mu_{0}\right) /\left(\lambda_{1}-\lambda_{0}\right), \\
& D_{2}=\left(\lambda_{0}+2 \mu_{0}\right) /\left(\lambda_{1}-\lambda_{0}\right), \\
& D_{2}=\lambda_{0} /\left(\lambda_{1}-\lambda_{0}\right)
\end{aligned}
$$


Furthermore, Eshelby's tensor [Eshelby, 1957] is defined as:

$$
\begin{aligned}
& S_{1111}=\frac{1}{2\left(1-\nu_{0}\right)}\left(1-2 \nu_{0}+\frac{3 \alpha^{2}-1}{\alpha^{2}-1}-\left(1-2 \nu_{0}+\frac{3 \alpha^{2}}{\alpha^{2}-1}\right) g\right) \\
& S_{2222}=S_{3333}=\frac{3}{8\left(1-\nu_{0}\right)} \frac{\alpha^{2}}{\alpha^{2}-1}+\frac{1}{4\left(1-\nu_{0}\right)}\left(1-2 \nu_{0}-\frac{9}{4\left(\alpha^{2}-1\right)}\right) g \\
& S_{2233}=S_{3322}=\frac{1}{4\left(1-\nu_{0}\right)}\left(\frac{\alpha^{2}}{2\left(\alpha^{2}-1\right)}-\left(1-2 \nu_{0}+\frac{3}{4\left(\alpha^{2}-1\right)}\right) g\right) \\
& S_{2211}=S_{3311}=-\frac{1}{2\left(1-\nu_{0}\right)} \frac{\alpha^{2}}{\alpha^{2}-1}+\frac{1}{4\left(1-\nu_{0}\right)}\left(\frac{3 \alpha^{2}}{\alpha^{2}-1}-\left(1-2 \nu_{0}\right)\right) g \\
& S_{1122}=S_{1133}=-\frac{1}{2\left(1-\nu_{0}\right)}\left(1-2 \nu_{0}+\frac{1}{\alpha^{2}-1}\right)+\frac{1}{2\left(1-\nu_{0}\right)}\left(1-2 \nu_{0}+\frac{3}{2\left(\alpha^{2}-1\right.}\right) g \\
& S_{2323}=S_{3232}=\frac{1}{4\left(1-\nu_{0}\right)}\left(\frac{\alpha^{2}}{2\left(\alpha^{2}-1\right)}+\left(1-2 \nu_{0}+\frac{3}{4\left(\alpha^{2}-1\right)}\right) g\right) \\
& S_{1212}=S_{1313}=\frac{1}{4\left(1-\nu_{0}\right)}\left(1-2 \nu_{0}-\frac{\alpha^{2}+1}{\alpha^{2}-1}-\frac{1}{2}\left(1-2 \nu_{0}-\frac{3\left(\alpha^{2}+1\right)}{\alpha^{2}-1}\right) g\right)
\end{aligned}
$$

with

$$
\begin{aligned}
& g=\frac{\alpha}{{\sqrt{\alpha^{2}-1}}^{3}}\left(\alpha \sqrt{\alpha^{2}-1}-\cosh ^{-1} \alpha\right) \text { or } \\
& g=\frac{\alpha}{{\sqrt{\alpha^{2}-1}}^{3}}\left(\cos ^{-1} \alpha-\alpha \sqrt{1-\alpha^{2}}\right) \quad \text { for disc-shaped inclusions. }
\end{aligned}
$$

\section{F.2 Thomsen parameter}

Thomsen [1986] introduced new notation to describe transverse isotropy. He uses five parameters, two elastic moduli (vertical P- and S-velocity) and three dimensionless measures of anisotropy, combined from elastic moduli. One of their advantages is that they reduce to zero in the isotropic case.

The vertical P- and S-velocities can be written as:

$$
\begin{aligned}
& \alpha_{0}=v_{P}\left(0^{\circ}\right)=\sqrt{\frac{c_{33}}{\rho}} \\
& \beta_{0}=v_{S V}\left(0^{\circ}\right)=v_{S H}\left(0^{\circ}\right)=\sqrt{\frac{c_{44}}{\rho}}
\end{aligned}
$$

The new three parameters are defined as:

$$
\begin{aligned}
\epsilon & =\frac{c_{11}-c_{33}}{2 c_{33}} \\
\gamma & =\frac{c_{66}-c_{44}}{2 c_{44}} \\
\delta^{*} & =\frac{1}{2 c_{33}^{2}}\left[2\left(c_{13}+c_{44}\right)^{2}-\left(c_{33}-c_{44}\right)\left(c_{11}+c_{33}-2 c_{44}\right)\right]
\end{aligned}
$$


The wave speeds can then be written as:

$$
\begin{aligned}
v_{P}(\theta) & =\alpha_{0} \sqrt{1+\epsilon \sin ^{2} \theta+D(\theta)}=\alpha_{0} a_{P}(\theta) \\
v_{S V}(\theta) & =\beta_{0} \sqrt{1+\frac{\alpha_{0}^{2}}{\beta_{0}^{2}} \epsilon \sin ^{2} \theta-\frac{\alpha_{0}^{2}}{\beta_{0}^{2}} D(\theta)}=\beta_{0} a_{S V}(\theta) \\
v_{S H}(\theta) & =\beta_{0} \sqrt{\left.1+2 \gamma \sin ^{2} \theta\right)}=\beta_{0} a_{S H}(\theta),
\end{aligned}
$$

with

$$
D(\theta)=\frac{1}{2} \sqrt{q^{2}+4 \delta \sin ^{2} \theta+4 \sin ^{4} \theta[\epsilon(q+e)-\delta]}-\frac{q}{2}
$$

and

$$
q=1-\frac{\beta_{0}^{2}}{\alpha_{0}^{2}}=\frac{c_{33}-c_{44}}{c_{33}}
$$

In the case of weak anisotropy and small phase angles, equation F.32 can be developed in a Taylor series. "Weak" anisotropy is defined as $\varepsilon, \gamma, \delta \ll 1$ or $10-20 \%$ [Thomsen, 1986]. Thus, equation F.31 reduces to:

$$
\begin{aligned}
v_{P}(\theta) & \approx \alpha_{0}\left(1+\delta \sin ^{2} \theta \cos ^{2} \theta+\epsilon \sin ^{4} \theta\right) \\
v_{S V}(\theta) & \approx \beta_{0}\left(1+\frac{\alpha_{0}^{2}}{\beta_{0}^{2}}(\varepsilon-\delta) \sin ^{2} \theta \cos ^{2} \theta\right) \\
v_{S H}(\theta) & \approx \beta_{0}\left(1+\gamma \sin ^{2} \theta\right)
\end{aligned}
$$

In the special case of vertical incidence $\left(\theta=0^{\circ}\right)$, these equations reduce to:

$$
\begin{aligned}
v_{P}\left(0^{\circ}\right) & \approx v_{P_{0}} \\
v_{S V}\left(0^{\circ}\right) & \approx v_{S_{0}} \\
v_{S H}\left(0^{\circ}\right) & \approx v_{S_{0}}
\end{aligned}
$$

And the special case of horizontal incidence $\left(\theta=90^{\circ}\right)$, these equations reduce to:

$$
\begin{aligned}
v_{P}\left(90^{\circ}\right) & \approx v_{P_{0}}(1+\varepsilon) \\
v_{S V}\left(90^{\circ}\right) & \approx v_{S_{0}} \\
v_{S H}\left(90^{\circ}\right) & \approx v_{S_{0}}(1+\gamma)
\end{aligned}
$$

This fifth elastic parameter $\delta^{*}$ can now be replaced with the simpler parameter $\delta$ :

$$
\delta=\frac{\left(c_{13}+c_{44}\right)^{2}-\left(c_{33}-c_{44}\right)^{2}}{2 c_{33}\left(c_{33}-c_{44}\right)}
$$

Here, $\theta$ is the angle between the wavefront normal and the symmetry axis and the parameters $\varepsilon$ and $\gamma$ are defined as the fractional differences between the P-and the S-wave 


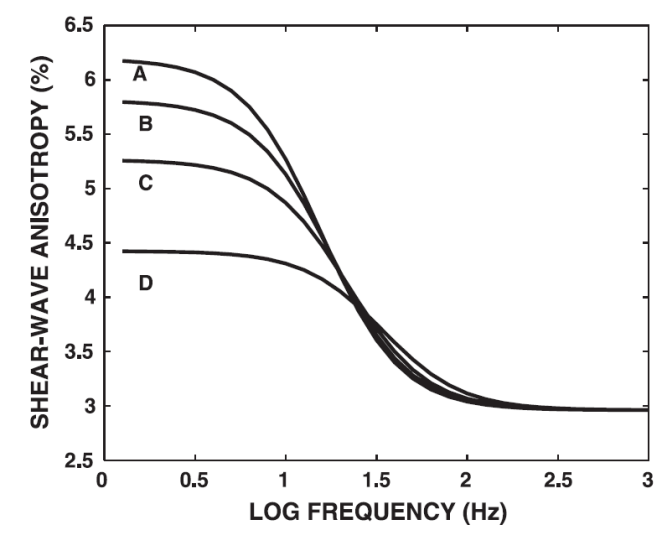

(a) Published in Chapman et al. [2003], Fig. 1

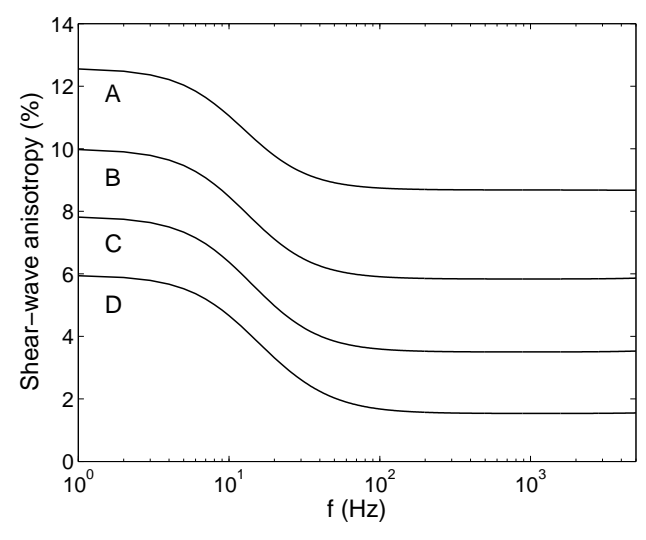

(b) Attempted reproduction

FIGURE F.1 Frequency dependent shear-wave anisotropy for propagation direction of $70^{\circ}$. Cracks filled with brine with varying micro-crack density $\varepsilon_{c}$ of A) $\left.0.08, B\right) 0.06$, C) $\left.0.04, D\right) 0.02$.

velocities, respectively, in the vertical and horizontal directions:

$$
\begin{aligned}
\varepsilon & =\frac{v_{P}\left(90^{\circ}\right)-v_{P}\left(0^{\circ}\right)}{v_{P}\left(0^{\circ}\right)} \\
\gamma & =\frac{v_{S H}\left(90^{\circ}\right)-v_{S V}\left(90^{\circ}\right)}{v_{S V}\left(90^{\circ}\right)}=\frac{v_{S H}\left(90^{\circ}\right)-v_{S H}\left(0^{\circ}\right)}{v_{S H}\left(0^{\circ}\right)}
\end{aligned}
$$

\section{F.3 Modelling frequency dependent anisotropy}

\section{F.3.1 Testing the Matlab ${ }^{\circledR}$ code}

This section describes the problem with the Matlab ${ }^{\circledR}$ code written for the frequency dependent analysis based on Chapman's papers [Chapman, 2003; Chapman et al., 2003] and shows calculations based on the code as a work in progress. The calculations in section 7.6.2 are based on a Fortran77 code, kindly provided by Mark Chapman (personal communication).

To test the Matlab ${ }^{\circledR}$ code, first calculations were run with input parameters given in Chapman [2003] and Chapman et al. [2003]. But the published results could not exactly be reproduced. The calculations in the code were checked by several reviewers, and a mistake could not be found. Instead, two misprints were discovered in the two publications by Chapman: Equation A.13 [Chapman et al., 2003] has a negative sign in the expression $3\left(1-K_{c}\right)$ in the denominator within the parentheses, while equation 37 [Chapman, 2003] has a positive sign in it. This should not be a concern, as $K_{c}=0$ is assumed. A second misprint gives a factor $4 / 5 \mu^{2}$ in equation A.16 [Chapman et al., 2003], while equation 58 [Chapman, 2003] has $4 / 15 \mu^{2}$. The input of either of the latter expression leads to higher velocities and anisotropies in the calculations. The notation from Chapman et al. [2003] is used for the fol- 


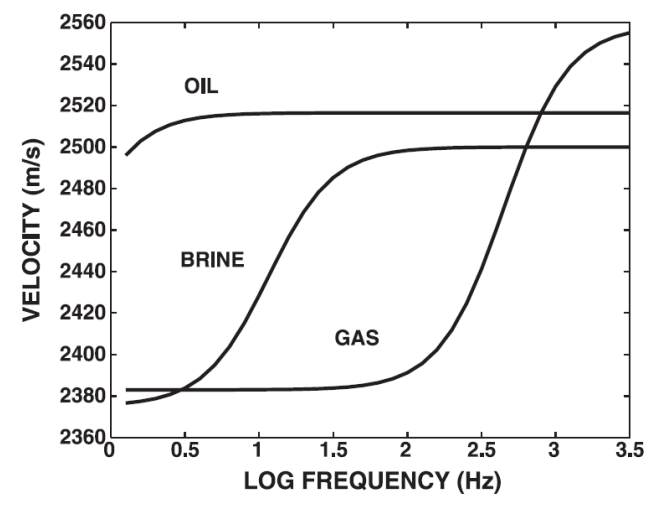

(a) Published in Chapman et al. [2003], Fig. 7

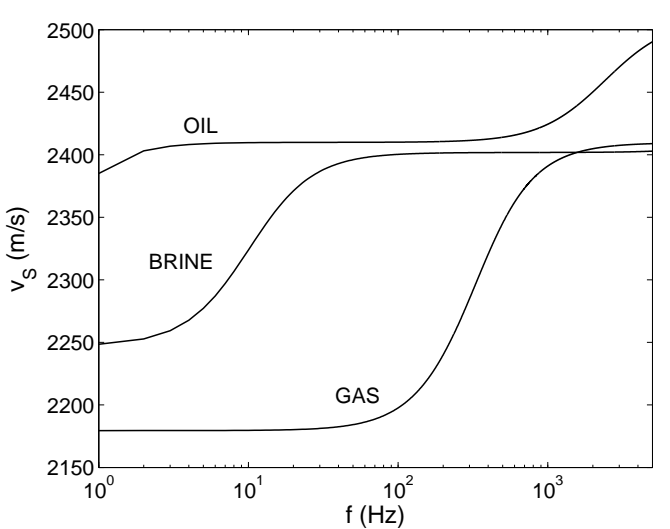

(b) Attempted reproduction

FIGURE F. 2 Frequency dependent quasi-shear velocity for propagation direction of $45^{\circ}$ for different crack fills, micro-crack density $\varepsilon_{c}=0.05$ and porosity $\phi_{p}=0.1$.

lowing calculations. An additional misprint stated by Chapman (personal communication) refers to the final diagram with the different attenuations (figure 10 in Chapman [2003]). It states that it has been calculated for $3 \mathrm{kHz}$, but was actually calculated for $30 \mathrm{kHz}$.

It cannot be ruled out that there is a misprint or misunderstanding in the way the input parameters are given in the publications or used here. Generally, the results from the Matlab ${ }^{\circledR}$ code show the expected trends (figures F.1 and F.2), but fail to reproduce the exact numbers. Published results (figure F.2(a)) show anisotropy for different crack densities falling to a common value at the highest frequencies. Every set of lines in the plots with different crack densities attempted to reproduce has lines that are subparallel to each other, never falling to a common value (figure F.2(b)). This occurs independently of the porosities used. A broader range in anisotropies, ranging from 2 to $13 \%$ for brine, is reproduced compared to the published values ranging from 3 to $6 \%$. The change in anisotropy occurs over the same frequency range as in published graphs. The graphs reproducing figure 7 (figure F.3(b)) have a similar shape, and the changes in velocity occur in the same frequency range as observed for the three different fluids in the original graphs (figure F.3(a)). But all the reproduced absolute velocities are lower than the published ones.

\section{F.3.2 Frequency dependent elastic parameters}

$$
\begin{aligned}
C_{1111}= & (\Lambda+2 \Upsilon) \\
& -\epsilon_{c}\left[\frac{8 L_{2}(1-\nu)}{3 \mu}+\frac{128}{45} \frac{1-\nu}{(2-\nu)} \mu-\frac{8 L_{2}(1-\nu)}{\mu} G_{1}-\frac{8 \kappa^{2}(1-\nu)}{\mu} G_{2}-\frac{8 \lambda \kappa(1-\nu)}{3 \mu} G_{3}\right] \\
& -\phi_{p}\left[\frac{3}{4 \mu} \frac{1-\nu}{1+\nu}\left(3 \lambda^{2}+4 \lambda \mu+\frac{36+20 \nu}{7-5 n u} \mu^{2}\right)-\left(1+\frac{3 \kappa}{4 \mu}\right)\left(3 \kappa D_{1}+\lambda D_{2}\right)\right] \\
& -\epsilon_{f}\left[\frac{8 \lambda^{2}(1-\nu)}{3 \mu}-\frac{8 \lambda \kappa(1-\nu)}{\mu} F_{1}-\frac{8 \lambda^{2}(1-\nu)}{3 \mu} F_{2}\right] \\
C_{3333}= & (\Lambda+2 \Upsilon)
\end{aligned}
$$




$$
\begin{aligned}
& -\epsilon_{c}\left[\frac{8 L_{2}(1-\nu)}{3 \mu}+\frac{128}{45} \frac{1-\nu}{(2-\nu)} \mu-\frac{8 L_{2}(1-\nu)}{\mu} G_{1}-\frac{8 \kappa^{2}(1-\nu)}{\mu} G_{2}-\frac{(8 \lambda+2 \mu) \kappa(1-\nu)}{3 \mu} G_{3}\right] \\
& -\phi_{p}\left[\frac{3}{4 \mu} \frac{1-\nu}{1+\nu}\left(3 \lambda^{2}+4 \lambda \mu+\frac{36+20 \nu}{7-5 \nu} \mu^{2}\right)-\left(1+\frac{3 \kappa}{4 \mu}\right)\left(3 \kappa D_{1}+(\lambda+2 \mu) D_{2}\right)\right] \\
& -\epsilon_{f}\left[\frac{(8 \lambda+2 \mu)^{2}(1-\nu)}{3 \mu}-\frac{8(\lambda+2 \mu) \kappa(1-\nu)}{\mu} F_{1}-\frac{(8 \lambda+2 \mu)^{2}(1-\nu)}{3 \mu} F_{2}\right] \\
C_{2323}= & \Upsilon \\
& -\epsilon_{c}\left[\frac{32}{45} \mu(1-\nu)\left(1-G_{1}\right)+\frac{32}{45} \frac{1-\nu}{(2-\nu)} \mu\right] \\
& -15 \phi_{p} \frac{1-\nu}{7-5 \nu} \mu \\
& -\epsilon_{f} \frac{16(1-\nu)}{3(2-\nu)} \mu \\
& \Lambda \\
& -\epsilon_{c}\left[\frac{8 L_{4}(1-\nu)}{\mu}-\frac{64}{45} \frac{1-\nu}{(2-\nu)} \mu-\frac{8 L_{4}(1-\nu)}{3 \mu} G_{1}-\frac{8 \kappa^{2}(1-\nu)}{\mu} G_{2}-\frac{8 \lambda \kappa(1-\nu)}{3 \mu} G_{3}\right] \\
& -\phi_{p}\left[\frac{3}{4 \mu} \frac{1-\nu}{1+\nu}\left(3 \lambda^{2}+4 \lambda \mu-\frac{4(1+5 \nu)}{7-5 \nu} \mu^{2}\right)-\left(1+\frac{3 \kappa}{4 \mu}\right)\left(3 \kappa D_{1}+\lambda D_{2}\right)\right] \\
& -\epsilon_{f}\left[\frac{8 \lambda^{2}(1-\nu)}{3 \mu}-\frac{8 \lambda \kappa(1-\nu)}{\mu} F_{1}-\frac{8 \lambda^{2}(1-\nu)}{3 \mu} F_{2}\right] \\
& \Lambda \\
& -\epsilon_{c}\left[\frac{8 L_{4}(1-\nu)}{\mu}-\frac{64}{45} \frac{1-\nu}{(2-\nu)} \mu-\frac{8 L_{4}(1-\nu)}{3 \mu} G_{1}-\frac{8 \kappa^{2}(1-\nu)}{\mu} G_{2}-\frac{8(\lambda+\mu) \kappa(1-\nu)}{3 \mu} G_{3}\right] \\
& -\phi_{p}\left[\frac{3}{4 \mu} \frac{1-\nu}{1+\nu}\left(3 \lambda^{2}+4 \lambda \mu+\frac{4(1+5 \nu)}{7-5 \nu} \mu^{2}\right)-\left(1+\frac{3 \kappa}{4 \mu}\right)\left(3 \kappa D_{1}+(\lambda+\mu) D_{2}\right)\right] \\
& -\epsilon_{f}\left[\frac{8 \lambda(\lambda+\mu)(1-\nu)}{3 \mu}-\frac{8(\lambda+\mu) \kappa(1-\nu)}{\mu} F_{1}-\frac{8 \lambda(\lambda+\mu)(1-\nu)}{3 \mu} F_{2}\right] \\
C_{1133} &
\end{aligned}
$$

The parameters $D_{1}, D_{2}, G_{1}, G_{2}, G_{3}, F_{1}, F_{2}, L_{2}, L_{3}$ and $L_{4}$ are given by:

$$
\begin{aligned}
D_{1} & =\frac{\frac{\iota}{3\left(1+K_{c}\right)}+(1-\iota) \gamma^{\prime}-\frac{i \omega \tau_{m}}{1+i \omega \tau_{m}}\left(\frac{1}{3\left(1+K_{c}\right)}-\gamma^{\prime}\right)\left(\iota+\frac{\iota \beta}{1+i \omega \tau_{f}}\right)}{(1-\iota) \gamma+\frac{(1-\iota) \beta}{1+i \omega \tau_{f}}+\left(\iota+\frac{\iota \beta}{1+i \omega \tau_{f}}\right)\left(\frac{1+i \omega \gamma \tau_{m}}{1+i \omega \tau_{m}}\right)} \\
D_{2} & =\frac{\frac{\beta}{\left(1+K_{c}\right)\left(1+i \omega \tau_{f}\right)}}{(1-\iota) \gamma+\frac{(1-\iota) \beta}{1+i \omega \tau_{f}}+\left(\iota+\frac{\iota \beta}{1+i \omega \tau_{f}}\right)\left(\frac{1+i \omega \gamma \tau_{m}}{1+i \omega \tau_{m}}\right)} \\
G_{1} & =\frac{i \omega \tau_{m}}{\left(1+K_{c}\right)\left(1+i \omega \tau_{m}\right)} \\
G_{2} & =\frac{1+i \omega \gamma \tau_{m}}{1+i \omega \tau_{m}} D_{1}-\frac{i \omega \tau_{m} \gamma^{\prime}}{1+i \omega \tau_{m}} \\
G_{3} & =\frac{1+i \omega \gamma \tau_{m}}{1+i \omega \tau_{m}} D_{2} \\
F_{1} & =\frac{1}{1+i \omega \tau_{f}}\left(\frac{1+i \omega \gamma \tau_{m}}{1+i \omega \tau_{m}} \iota D_{1}+(1-\iota) D_{1}+\frac{i \iota \omega \tau_{m}}{1+i \omega \tau_{m}}\left(\frac{1}{3\left(1+K_{c}\right)}-\gamma^{\prime}\right)\right) \\
F_{2} & \left.=\frac{1}{1+i \omega \tau_{f}}\left(\frac{i \omega \tau_{f}}{1+K_{c}}+\iota \frac{1+i \omega \gamma \tau_{m}}{1+i \omega \tau_{m}} D_{2}+(1-\iota) D_{2}\right)\right) \\
L_{2} & =\lambda^{2}+\frac{4}{3} \lambda \mu+\frac{4}{5} \mu^{2} \\
L_{3} & =4\left(\lambda^{2}+\frac{4}{3} \lambda \mu+\frac{8}{15} \mu^{2}\right) \\
L_{4} & =\lambda^{2}+\frac{4}{3} \lambda \mu+\frac{4}{15} \mu^{2}
\end{aligned}
$$


Further parameters are defined as the following:

$$
\begin{aligned}
& \text { Critical stress } \quad \sigma_{c}=\frac{\pi \mu r}{2(1-\nu)} \\
& \text { Bulk modulus } \quad \kappa=\lambda+\frac{2}{3} \mu \\
& \phi_{f}=\frac{4}{3} \epsilon_{f} \pi r \\
& \text { Volume fraction of fractures } \quad \phi_{c}=\frac{4}{3} \epsilon \pi r \\
& \text { Volume fraction of cracks } \quad=\frac{4}{3} \pi a_{f}^{3} r \\
& \text { Volume of fractures } \quad f_{v}=\frac{\iota \phi_{f}}{\phi_{c}} \\
& K_{c}=\frac{\sigma_{c}}{\kappa_{f}} \\
& K_{p}=\frac{4 \mu}{3 \kappa_{f}} \\
& \iota=\frac{4}{3} \pi \epsilon \\
& \frac{4}{3} \pi \epsilon+\phi_{p} \\
& \gamma^{\prime}=\frac{3 \pi}{8(1-\nu)}\left[1+\frac{4 \rho}{3 \rho_{f}}\left(\frac{v_{s}}{v_{f}}\right)^{2}\right] \\
& \gamma^{\prime}=\gamma \frac{1-\nu}{1+\nu} \frac{1}{1+K p}
\end{aligned}
$$




\section{APPENDIX G}

\section{Two-layer modelling}

\section{G.1 Horizontal symmetry axis}

Silver \& Savage [1994] introduce a method to calculate apparent splitting parameters for two horizontal vertical isotropic layers (horizontal symmetry axis). Characteristic for this two-layer case is the $\pi / 2$ periodicity of the apparent splitting parameters as a function of incoming polarisation. The apparent delay time varies with incoming polarisation in a nonintuitive way. In the special case of two layers with parallel fast polarisation, the apparent delay time is the sum of the two single delay times. If the two fast polarisations are approximately perpendicular, the delay times subtract. An extended version of Silver \& Savage [1994] method is applied here [Savage \& Silver, 1994]. It performs a grid search to find the best two-layer model fitting the observed data points. The search is performed over the $180^{\circ}$-range of fast polarisations with increments of $10^{\circ}$ and over delay times up to $4 \mathrm{~s}$ with increments of $0.2 \mathrm{~s}$. The best fit is defined by an L1 norm misfit measure, in order not to weight outliers as heavily as least-squares (L2). The minimum misfit can be found for either the fast polarisation $\Phi$, the delay time $\delta t$, or the total fit. The total fit is evaluated as the sum of the single fits, normalised by their standard deviations. Calculations depend on the dominant frequency of the waveform.

All the good and fair splitting measurements (table E.1), excluding null measurements are used for the grid search. First, the grid search is performed for the complete data set using an average frequency of $0.1 \mathrm{~Hz}$ ( $c f$. spectra in section D.2).

The best-fit models minimising the misfit for delay time, fast polarisation, and the total misfit consistently give nearly parallel fast polarisation in both layers and delay times between 0.8 and $1.2 \mathrm{~s}$ in each layer (figure G.2). These simple models cannot match all of the measurements. Especially a group of measurement with initial polarisation between 60 and $90^{\circ}$ with large delay times and differing fast polarisation cannot be matched with a single two-layer model for the whole of North Island. Similarly, the apparent systematic variation of fast polarisations (figure G.2) is not fitted. An additional test model (alltest, table G.1), 


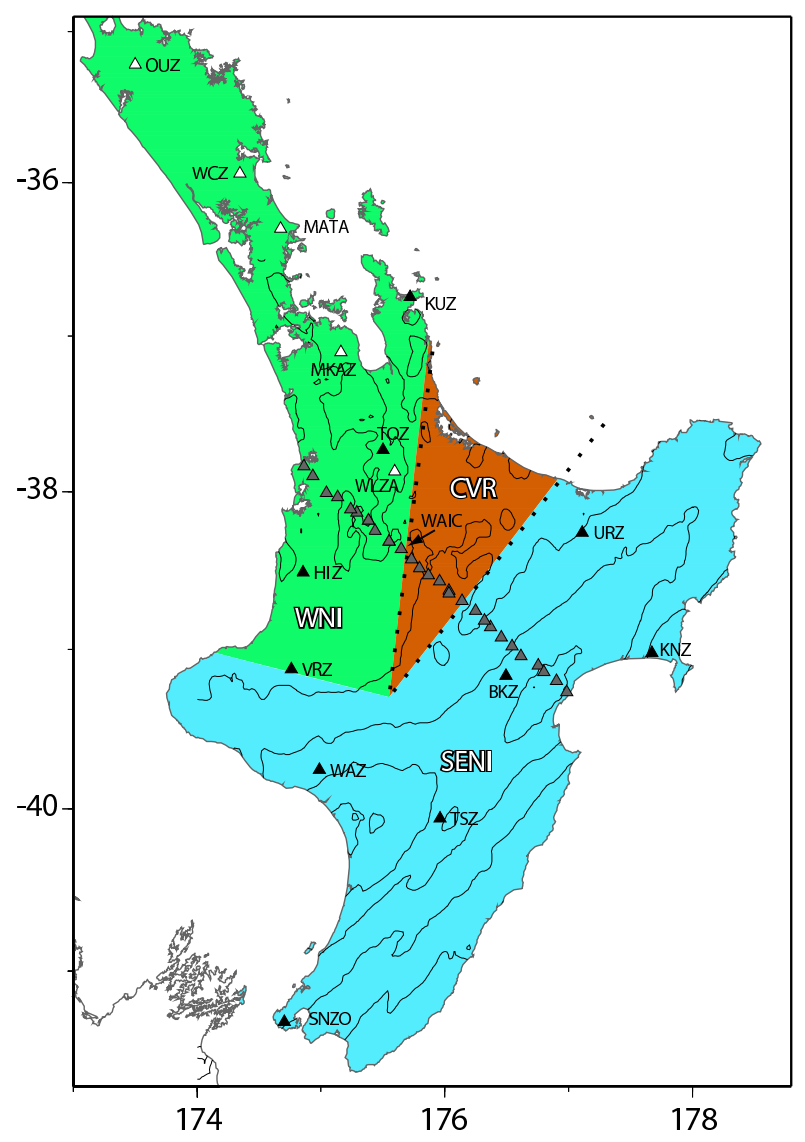

TABLE G.0 Stations included in the different anisotropic regions used for anisotropic modelling

\begin{tabular}{|c|}
\hline CVR \\
TAUC, TUKC, POIC, KIWC, \\
TETC, LDEN, SCHC \\
\hline WNI \\
WAIC, YUPC, MILC, WULC, \\
PUKC, PUKC, STEC, YTFC, \\
VERC, FLYC, PEAC \\
TOZ, HIZ, WLZA, KUZ \\
\hline CVR2 \\
TAUC, TUKC, POIC, KIWC, \\
TETC, LDEN, SCHC, WAIC \\
\hline WNI2 \\
YUPC, MILC, WULC, PUKC, \\
PUKC, STEC, YTFC, VERC, \\
FLYC, PEAC \\
TOZ, HIZ, WLZA, KUZ \\
\hline SENI \\
all but CVR(2) and WNI(2) \\
\hline
\end{tabular}

FIGURE G.1 Anisotropic regions used for two-layer modelling. Black triangles indicate permanent GeoNet station, grey triangles temporary (W)CNIPSE stations and white triangles temporary stations from other deployments. Station WAIC is modelled as part of the CVR in the first model variation (CVR, WNI) and as part of western North Island (WNI) in the second variation (CVR2, WNI2) (q.v. table G.1). SENI=south Eastern North Island.

excluding all measurement with fast polarisations $\Phi>80^{\circ}$ does lead to exactly the same best-fit models, with smaller errors.

The confidence regions (figure G.3) show that the two fast polarisations are well constrained against each other, ranging between 20 and $50^{\circ}$ and therefore implying an accuracy of $\pm 15^{\circ}$. The two parameters, delay time and fast polarisation, are well constrained within the same layer. They show the same accuracy of $\pm 15^{\circ}$ for delays greater than about $0.5 \mathrm{~s}$, with a $90^{\circ}$-ambiguity. But the different parameters are not constrained across layers. This means that the two layers are interchangeable and therefore independent. Because the splitting parameters for both layers are almost identical, this leads to the conclusion that the best-fit solution for the complete data set is in fact a single layer model.

The wide spread of the data in the mentioned polarisation range makes it hard to find a good fit with only one two-layer model. A new modelling approach splits the data into the three different anisotropic regions (figure G.1) identified by the shear-wave splitting analysis (cf. section 5.5.1). Two ways of dividing the data are applied. The first grouping follows strictly the boundaries of the CVR, so that the CNIPSE stations TAUC to SCHC belong to the 

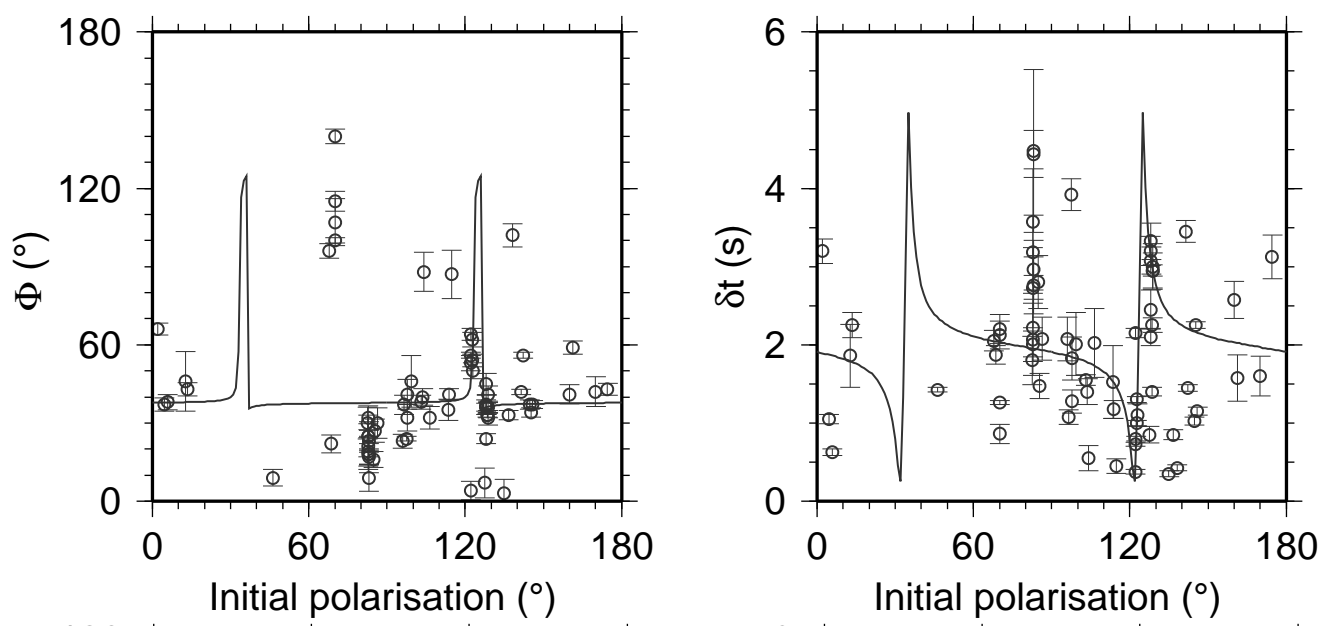

Best $\Phi$ (left) $\Phi 1=30.008 t 1=1.00$
$\Phi 2=40.008 \mathrm{~s} 2=1.20$
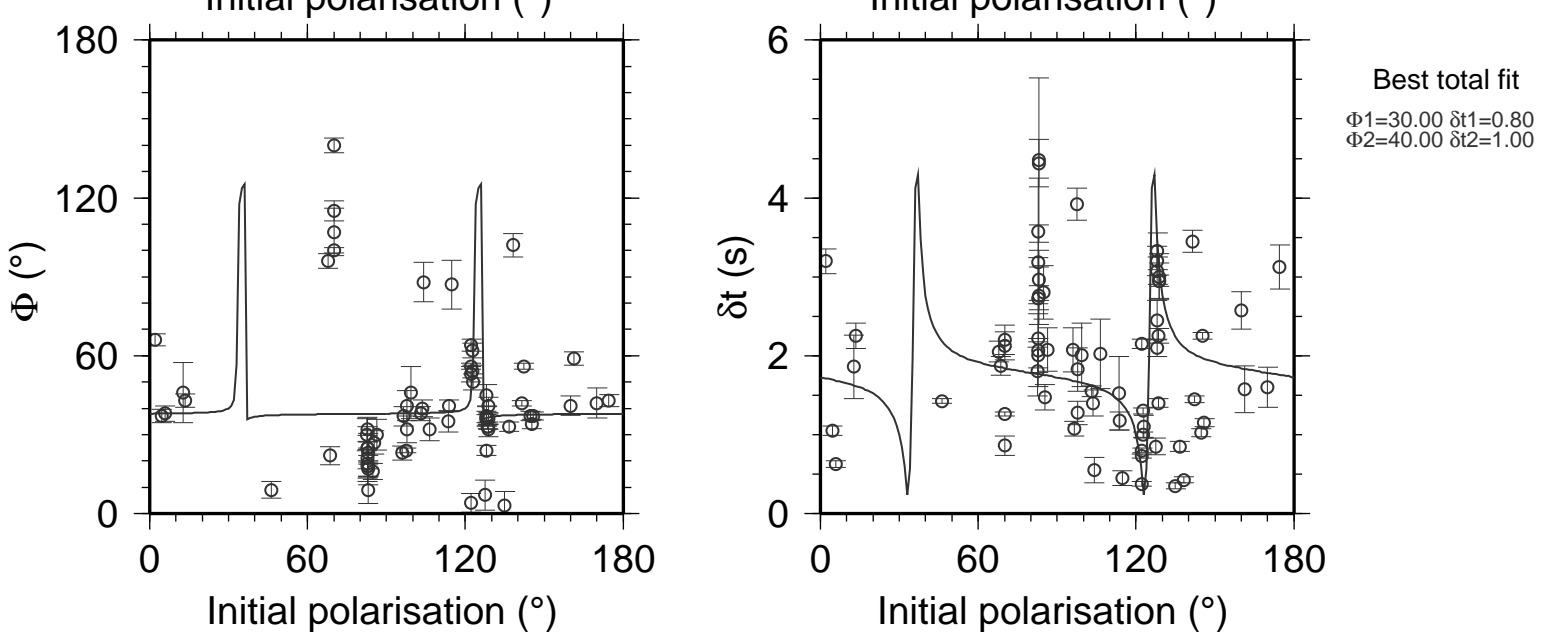

FIGURE G.2 Best-fit two-layer models for whole North Island. Lines show the expected results for the two-layer model with best fit to all teleseismic shear-wave splitting measurements for North Island, shown as circles. Top: Best-fit results to fast polarisation $\Phi$ only (left) and delay times $\delta$ tonly (right). Bottom: Best total fit $(\Phi$ and $\delta t)$.

region of the Central Volcanic Region (CVR) and stations WAIC to PEAC along the CNIPSE line belong to western North Island (WNI) (table G.1). All the remaining stations are included in the third region, in the following called South-Eastern North Island (SENI). The second version of the regional model moves station WAIC from WNI (now called WNI2) into the CVR (now called CVR2). This modified partitioning includes only stations where no splitting is apparent into western North Island and stations with apparent splitting in the CVR and the southeastern North Island, respectively. As the new partitioning only affects stations on the western boundary of the CVR, region SENI and stations therein stay the same for both versions. The grid search is performed for data from the different regions separately. Again, the dominant frequency is set to $0.1 \mathrm{~Hz}$.

The results for the two version of the "regional" model with different partitioning show only minor differences in the best-fit models for CVR and WNI (table G.1). Overall, the fit of the data points to the model improved slightly by introducing the three regions. A more detailed comparison including statistical evaluations is disregarded because of the similarity 

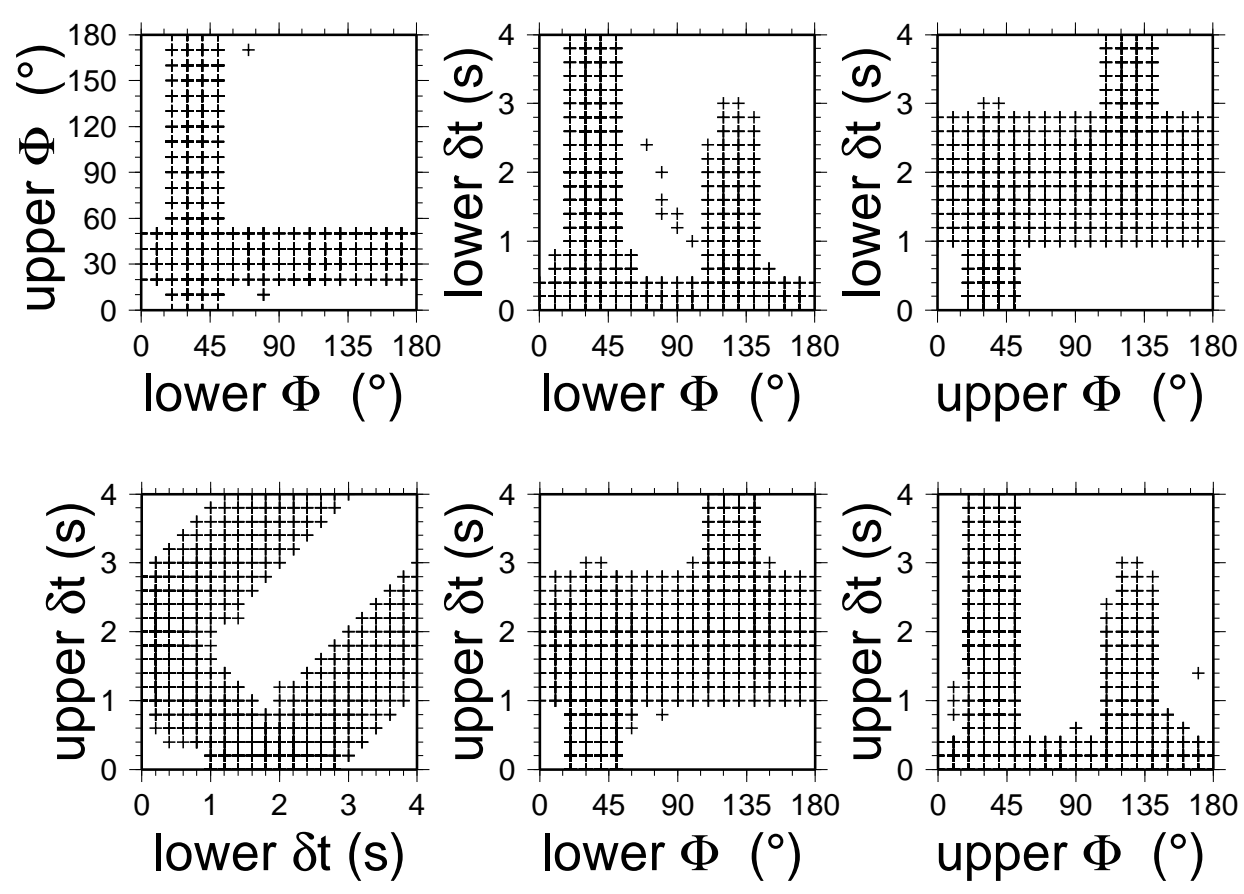

FIGURE G.3 95\% confidence regions for best-fit model with horizontal symmetry axis for the full data set (figure G.2). Crosses represent solution within the confidence region.

of results. Figure G.4 shows the best-fit model results in comparison with the data for the second version of the regional model.

Results for WNI with best fit for fast polarisation and best total fit agree with only a $0.2 \mathrm{~s}$ difference in delay time and the order of the layers interchanged. Fast polarisations in the two layers for both models are perpendicular with one layer showing north-south fast polarisation and the other east-west. Fast polarisations for the best $\delta t$ fit model are almost perpendicular, but about $30^{\circ}$ different from the other two models. For the CVR, the three solutions for the different best fits do not agree in any of the layer parameters. The best model to fit the fast polarisation suggests low delay times of $0.2 \mathrm{~s}$ in both layers. On the other hand, the best-fit model for the delay time and the best total fit model require delay times of $1.4 \mathrm{~s}$ or $1.8 \mathrm{~s}$ in the first layer and $1.8 \mathrm{~s}$ or $1.0 \mathrm{~s}$ in the second, with nearly parallel fast polarisation. The best two-layer models for the SENI are the same as the results from the first model for all the data.

A third approach models the data by separating them into different phases to consider their different dominant frequencies. S- and SK(K)S-phases are modelled with a dominant frequency of $0.1 \mathrm{~Hz}$, whereas ScS-phases are modelled with a dominant frequency of $0.5 \mathrm{~Hz}$. Results for these "phase" models with the three different fit criteria vary widely. No consistent models could be found within any of the results. Most of the models prefer large differences in fast polarisations and delay times for the two layers (table G.1).

The last modelling approach combines the regional and the phase models described above. It looks for the best model to fit single phase data in the three different regions. Results for 

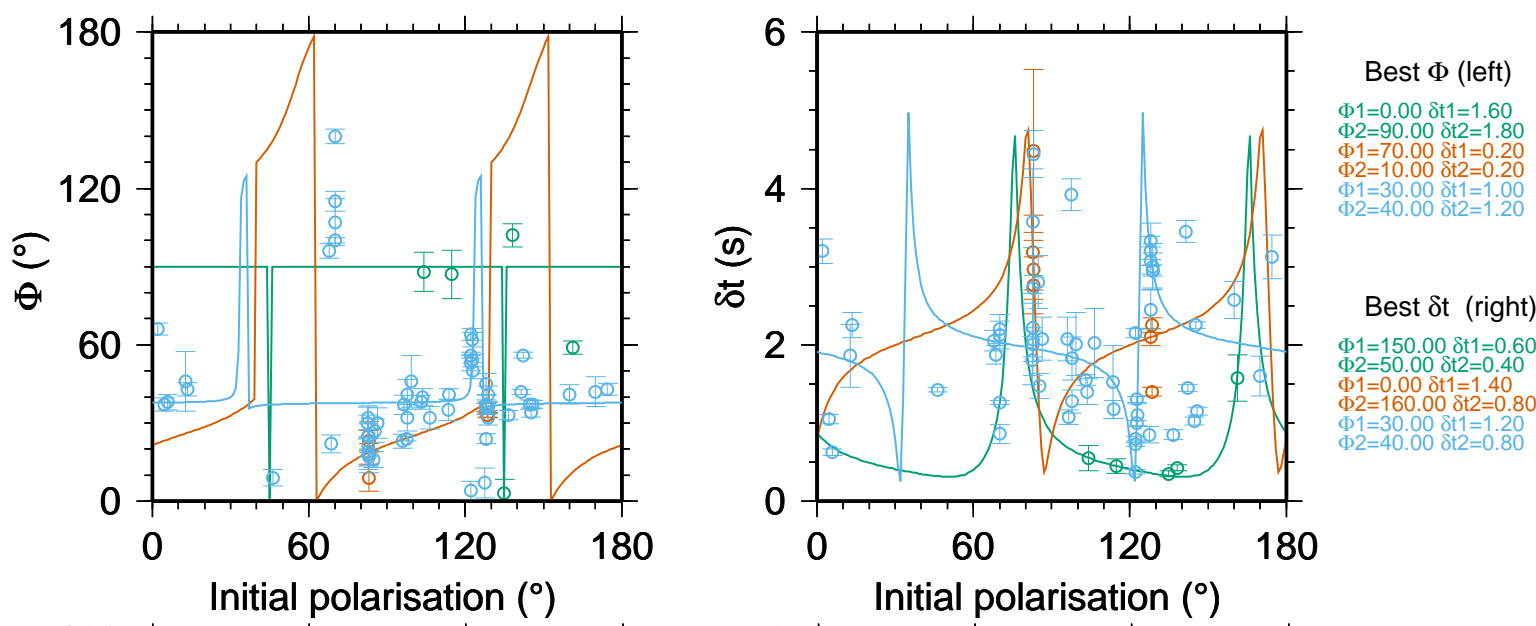

Best $\delta$ t (right)

$\Phi 1=150.00$ st $1=0.60$ $\Phi 2=50.00 \delta \mathrm{t} 2=0.40$ $\Phi 1=0.00 \delta \mathrm{t} 1=1.40$
$\Phi 2=160.00 \mathrm{st} 2=0.80$ $\Phi 2=160.00 \delta \mathrm{t} 2=0.80$
$\Phi 1=30.00 \delta \mathrm{t} 1=1.20$ $\Phi 2=40.008 \mathrm{t} 2=0.80$
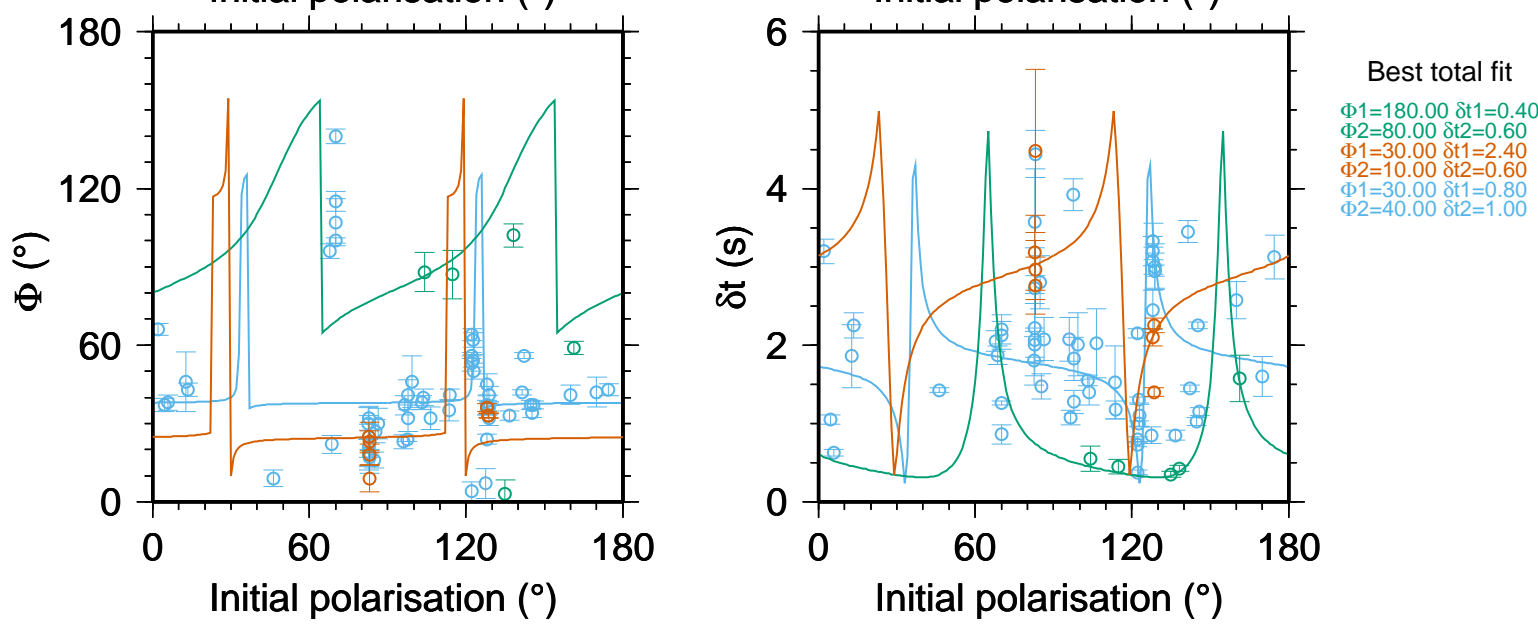

FIGURE G.4 Best-fit two-layer models for three anisotropic regions in North Island: Western North Island (green), CVR (orange) and southeastern North Island (blue). Coloured lines show the expected results for the best-fit two-layer model for teleseismic shear-wave splitting measurements in the three regions, shown as coloured circles. Top: Best-fit results for fast polarisation $\Phi$ only (left) and delay times $\delta$ t only (right). Bottom: Best total fit ( $\Phi$ and $\delta t$ ).

the SKS-phases in all three regions are comparable to results from the regional model including all phases. Differences between the three best-fit models are small. For ScS-phases, the best total fit and the best $\Phi$ fit model for WNI consists of two perpendicular layers. The best total fit and the best $\delta t$ fit models for the SENI region prefer that one of the layers has zero delay time. No ScS-phases were measured in the CVR; therefore, there is no such model for the CVR. Models for S-phases in the CVR vary widely. But the best total fit model gives the same fast polarisation of $30^{\circ}$ and $40^{\circ}$ as found before with the most general model for the complete data set. For the SENI model, results for the best $\delta t$ fit model and the best total fit model are similar. With 40 to $60^{\circ}$ the fast polarisations are also similar to the best overall model. There are no ScS-phase measurements for the CVR. 
Table G.1: Two-layer modelling results - horizontal symmetry. The index "1" denotes the first layer the wave passes through, e.g. the lower layer and the index "2" the second. The mean absolute error is the absolute error divided by the number of points to fit.

\begin{tabular}{|c|c|c|c|c|c|c|c|}
\hline Model & $\begin{array}{l}\text { Best } \\
\text { fit for }\end{array}$ & $\begin{array}{l}\Phi_{1} \\
\left({ }^{\circ}\right)\end{array}$ & $\begin{array}{l}\Phi_{2} \\
\left({ }^{\circ}\right)\end{array}$ & $\begin{array}{l}\delta t_{1} \\
\text { (s) }\end{array}$ & $\begin{array}{l}\delta t_{2} \\
\text { (s) }\end{array}$ & $\begin{array}{l}\text { Points } \\
\text { to fit }\end{array}$ & $\begin{array}{c}\text { Mean absolute } \\
\text { error }\end{array}$ \\
\hline all & $\overline{\Phi \Phi}$ & 30 & 40 & $\overline{1.0}$ & 1.2 & 69 & 16.9 \\
\hline all & $\delta t$ & 30 & 40 & 1.2 & 0.8 & 69 & 0.7 \\
\hline all & total & 30 & 40 & 0.8 & 1.0 & 69 & 55.3 \\
\hline all test & $\Phi$ & 30 & 40 & 1.0 & 1.2 & 61 & 10.4 \\
\hline all test & $\delta t$ & 30 & 40 & 1.2 & 0.8 & 61 & 0.7 \\
\hline all test & total & 30 & 40 & 0.8 & 1.0 & 61 & 42.1 \\
\hline CVR & $\Phi$ & 70 & 10 & 0.2 & 0.2 & 6 & 4.8 \\
\hline CVR & $\delta t$ & 0 & 160 & 1.4 & 0.8 & 6 & 0.3 \\
\hline CVR & total & 30 & 20 & 1.6 & 1.2 & 6 & 6.8 \\
\hline WNI & $\Phi$ & 180 & 90 & 0.2 & 1.4 & 6 & 14.0 \\
\hline WNI & $\delta t$ & 150 & 50 & 0.6 & 0.4 & 6 & 0.2 \\
\hline WNI & total & 180 & 90 & 0.2 & 1.2 & 6 & 12.7 \\
\hline CVR2 & $\Phi$ & 70 & 10 & 0.2 & 0.2 & 7 & 5.0 \\
\hline CVR2 & $\delta t$ & 0 & 160 & 1.4 & 0.8 & 7 & 0.4 \\
\hline CVR2 & total & 30 & 20 & 1.8 & 1.0 & 7 & 8.3 \\
\hline WNI2 & $\Phi$ & 90 & 180 & 1.4 & 0.4 & 5 & 10.2 \\
\hline WNI2 & $\delta t$ & 150 & 50 & 0.6 & 0.4 & 5 & - \\
\hline WNI2 & total & 180 & 90 & 0.2 & 1.2 & 5 & 8.4 \\
\hline SENI & $\Phi$ & 30 & 40 & 1.0 & 1.2 & 57 & 15.2 \\
\hline SENI & $\delta t$ & 30 & 40 & 1.2 & 0.8 & 57 & 0.6 \\
\hline SENI & total & 30 & 40 & 0.8 & 1.0 & 57 & 44.2 \\
\hline $\mathrm{S}$ & $\Phi$ & 110 & 20 & 0.2 & 0.2 & 16 & 13.7 \\
\hline$S$ & $\delta t$ & 60 & 50 & 0.6 & 2.0 & 16 & 0.4 \\
\hline$S$ & total & 50 & 40 & 0.2 & 2.0 & 16 & 23.9 \\
\hline SKS & $\Phi$ & 100 & 30 & 0.2 & 1.2 & 31 & 14.1 \\
\hline SKS & $\delta t$ & 80 & 160 & 2.0 & 0.2 & 31 & 0.7 \\
\hline SKS & total & 80 & 30 & 0.2 & 2.0 & 31 & 29.6 \\
\hline ScS & $\Phi$ & 30 & 130 & 0.4 & 1.6 & 22 & 15.9 \\
\hline $\mathrm{ScS}$ & $\delta t$ & 60 & 150 & 0.4 & 1.4 & 22 & 0.4 \\
\hline ScS & total & 130 & 40 & 0.4 & 1.2 & 22 & 24.9 \\
\hline CVRS & $\Phi$ & 30 & 40 & 0.8 & 0.2 & 2 & 1.5 \\
\hline CVRS & $\delta t$ & 110 & 160 & 1.8 & 1.6 & 2 & - \\
\hline CVRS & total & 120 & 10 & 1.6 & 0.8 & 2 & 1.0 \\
\hline CVRSKS & $\Phi$ & 170 & 0 & 1.0 & 1.2 & 4 & 5.3 \\
\hline CVRSKS & $\delta t$ & 180 & 170 & 0.8 & 1.6 & 4 & 0.5 \\
\hline CVRSKS & total & 10 & 20 & 1.4 & 2.0 & 4 & 4.3 \\
\hline CVRScS & - & - & - & - & - & 0 & - \\
\hline WNIS & $\Phi$ & 100 & 150 & 1.4 & 1.4 & 1 & - \\
\hline WNIS & $\delta t$ & 0 & 0 & 0.0 & 1.4 & 1 & - \\
\hline WNIS & total & 150 & 40 & 0.4 & 0.8 & 1 & - \\
\hline WNISKS & $\Phi$ & 50 & 60 & 0.6 & 1.8 & 1 & - \\
\hline
\end{tabular}


Table G.1: continued

\begin{tabular}{|c|c|c|c|c|c|c|c|}
\hline Model & $\begin{array}{c}\text { Best } \\
\text { fit for }\end{array}$ & $\begin{array}{c}\Phi_{\mathbf{1}} \\
\mathbf{(}^{\circ}\end{array}$ & $\begin{array}{c}\Phi_{\mathbf{2}} \\
\mathbf{(}^{\mathbf{}} \mathbf{c}\end{array}$ & $\begin{array}{c}\boldsymbol{\delta} \boldsymbol{t}_{\mathbf{1}} \\
\mathbf{( s )}\end{array}$ & $\begin{array}{c}\boldsymbol{\delta} \boldsymbol{t}_{\mathbf{2}} \\
\mathbf{( s )}\end{array}$ & $\begin{array}{c}\text { Points } \\
\text { to fit }\end{array}$ & $\begin{array}{c}\text { Mean absolute } \\
\text { error }\end{array}$ \\
\hline \hline WNISKS & $\delta t$ & 150 & 80 & 2.0 & 0.2 & 1 & - \\
WNISKS & total & 50 & 60 & 0.4 & 1.0 & 1 & - \\
WNIScS & $\Phi$ & 0 & 90 & 0.8 & 1.8 & 4 & 5 \\
WNIScS & $\delta t$ & 30 & 70 & 1.6 & 1.6 & 4 & - \\
WNIScS & total & 180 & 90 & 0.2 & 0.8 & 4 & 2.5 \\
\hline CVR2S & $\Phi$ & 30 & 100 & 2.0 & 0.2 & 3 & 1.0 \\
CVR2S & $\delta t$ & 70 & 160 & 0.4 & 2.0 & 3 & - \\
CVR2S & total & 30 & 40 & 1.2 & 0.4 & 3 & 3.0 \\
CVR2SKS & $\Phi$ & 170 & 0 & 1.0 & 1.2 & 4 & 5.3 \\
CVR2SKS & $\delta t$ & 180 & 170 & 0.8 & 1.6 & 4 & 0.5 \\
CVR2SKS & total & 10 & 20 & 1.4 & 2.0 & 4 & 4.3 \\
CVR2ScS & - & - & - & - & - & 0 & - \\
WNI2S & - & - & - & - & - & 0 & - \\
WNI2SKS & $\Phi$ & 50 & 60 & 0.6 & 1.8 & 1 & - \\
WNI2SKS & $\delta t$ & 150 & 80 & 2.0 & 0.2 & 1 & - \\
WNI2SKS & total & 50 & 60 & 0.4 & 1.0 & 1 & - \\
WNI2ScS & $\Phi$ & 0 & 90 & 0.8 & 1.8 & 4 & 5.0 \\
WNI2ScS & $\delta t$ & 30 & 70 & 1.6 & 1.6 & 4 & - \\
WNI2ScS & total & 180 & 90 & 0.2 & 0.8 & 4 & 2.5 \\
\hline SENIS & $\Phi$ & 110 & 20 & 0.2 & 0.2 & 13 & 15.9 \\
SENIS & $\delta t$ & 60 & 50 & 0.6 & 2.0 & 13 & 0.3 \\
SENIS & total & 50 & 40 & 1.4 & 1.0 & 13 & 21.1 \\
SENISKS & $\Phi$ & 50 & 30 & 0.6 & 2.0 & 26 & 13.8 \\
SENISKS & $\delta t$ & 30 & 40 & 0.4 & 1.6 & 26 & 0.6 \\
SENISKS & total & 0 & 30 & 0.0 & 2.0 & 26 & 25.9 \\
SENIScS & $\Phi$ & 30 & 130 & 0.4 & 1.6 & 18 & 8.0 \\
SENIScS & $\delta t$ & 0 & 0 & 0.0 & 1.2 & 18 & 0.4 \\
SENIScS & total & 0 & 40 & 0.0 & 1.2 & 18 & 16.8 \\
\hline
\end{tabular}

\section{G.2 Dipping symmetry axis}

In this section the modelling is extended in allowing for one more degree of freedom by introducing dipping symmetry axes. A single layer with dipping symmetry axis will exhibit symmetric (but not periodic) variations of splitting parameters with backazimuth and is the most general case of lateral variations for a hexagonal system [Silver \& Savage, 1994]. Hartog \& Schwartz [2000] introduce a method to perform a grid search over olivine a-axis orientations and path length. The maximum path length for each layer searched is $400 \mathrm{~km}$. Fast polarisations are searched from 0 to $180^{\circ}$-range and dips of the a-axis from -90 to $90^{\circ}$, measured upward from the horizontal (figure 3.2). The code solves the Christoffel equation for the propagation direction with a hexagonal elastic tensor derived from olivine plus an isotropic component [Hartog \& Schwartz, 2000]. As the code only allows the input of fixed incidence angles for the phases, they are fixed to averages of $22^{\circ}$ for S-, $10^{\circ}$ for SKS- and $7^{\circ}$ for 


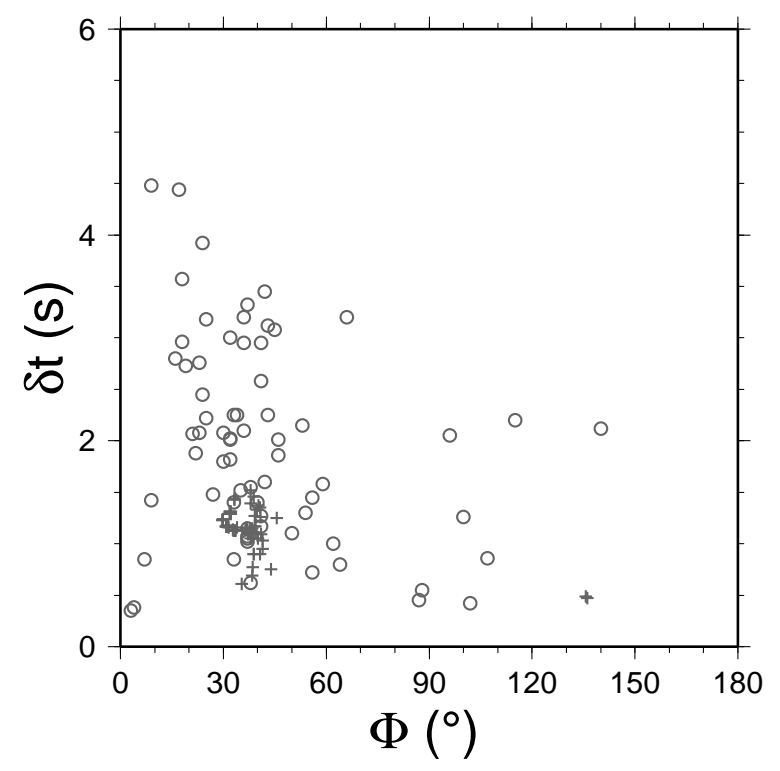

(a) Full data set

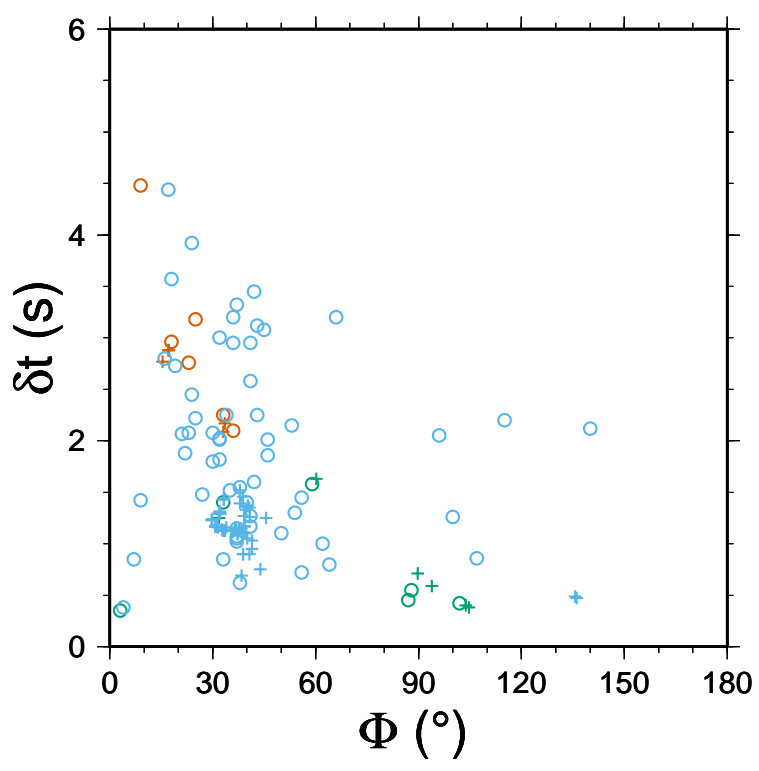

(b) Three anisotropic regions

FIGURE G.5 Results for two-layer modelling with dipping symmetry. Comparison of observed measurements (circles) with predicted measurements for the best solution of the two-layer dipping symmetry model (crosses) (a) for the full data set and (b) for the suggested three different regions: CVR (orange), WNI (green) and SENI (blue). For a perfect fit the crosses have to match the circles.

ScS-phases. The best-fit solution is found by comparing the observed and predicted splitting parameters and finding the minimum summed $\chi^{2}$ for both parameters. This method is combined with the two-layer grid search [Silver \& Savage, 1994; Marson-Pidgeon \& Savage, 2004], as described and used in the previous section. The minimum normalised total misfit for fast polarisation and delay time defines the best-fit model.

Analogously to the modelling with horizontal symmetry axis, the grid search is performed for the full data set and for the sub-data sets corresponding to the three different anisotropic regions, i.e. WNI, CVR and SENI. The best-fit two-layer model with dipping symmetry axis for the total data set, excluding null measurements, requires the fast axes in the two layers to be perpendicular. The upper layer is $40 \mathrm{~km}$ thick with a dip of $40^{\circ}$ and the lower layer is much thicker, $280 \mathrm{~km}$, with horizontal symmetry axis. Figure G.6(a) shows the fit of the measured data points (circles) to the predicted measurements from the best-fit model (crosses). Not many data points are matched well and especially the high delay times cannot be fitted with the suggested two-layer model.

The separation of the results into the three regions does not change the single layer solutions significantly (figure G.6(b)). The best solutions for SENI stay consistent with the solution for the full data set. For CVR, the best-fit solution requires long travel paths and steep dips. The top layer for WNI is consistent for both versions of the modelling, but the lower layer differs considerably. Note, that because of the almost perpendicular fast polarisations in model WNI, the dips of the layers are not subparallel. Again, the overall fit is 


\section{Lower layer}
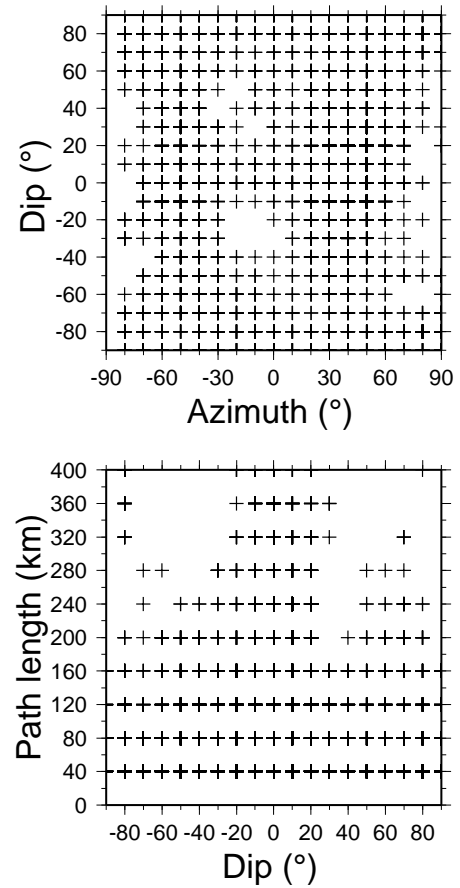

\section{Upper layer}
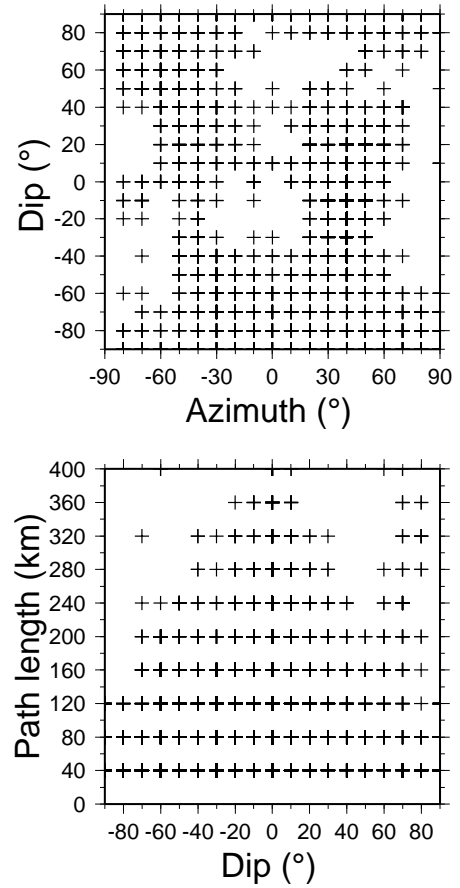

FIGURE G.6 95\% confidence region for the best-fit two-layer dipping symmetry axis model for the full data set (figure G.5). Crosses represent solution within the confidence region.

TABLE G.2 Two-layer modelling results - dipping symmetry. "Error" is the mean normalised error, i.e. the normalised error divided by the number of data points.

\begin{tabular}{|c|c|c|c|c|c|c|c|c|c|c|}
\hline \multirow[t]{2}{*}{ Model } & \multirow{2}{*}{$\begin{array}{l}\Phi_{1} \\
\left({ }^{\circ}\right)\end{array}$} & \multirow{2}{*}{$\begin{array}{c}\operatorname{dip}_{1} \\
\left.\mathbf{(}^{\circ}\right)\end{array}$} & \multirow{2}{*}{$\begin{array}{c}L_{1} \\
(\mathbf{k m})\end{array}$} & \multirow{2}{*}{$\begin{array}{l}\Phi_{2} \\
\left({ }^{\circ}\right)\end{array}$} & \multirow{2}{*}{$\begin{array}{c}\operatorname{dip}_{2} \\
\left({ }^{\circ}\right)\end{array}$} & \multirow{2}{*}{$\begin{array}{c}L_{2} \\
(\mathbf{k m})\end{array}$} & \multicolumn{3}{|c|}{ Error } & \multirow{2}{*}{$\begin{array}{l}\text { Data } \\
\text { points }\end{array}$} \\
\hline & & & & & & & $\bar{\Phi}$ & $\delta t$ & total & \\
\hline all & 40 & $\overline{0}$ & 280 & $\overline{130}$ & $\overline{-440}$ & 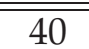 & 6.1 & 6.4 & $\bar{~} 12.5$ & "69 \\
\hline CVR & 20 & -30 & 360 & 150 & -80 & 360 & 1.2 & 0.5 & 1.8 & 6 \\
\hline WNI & 0 & -40 & 80 & 90 & 50 & 120 & 3.0 & 1.3 & 4.3 & 6 \\
\hline SENI & 40 & 0 & 280 & 130 & -40 & 40 & 6.4 & 6.6 & 13.0 & 57 \\
\hline CVR2 & 50 & -50 & 320 & 20 & -60 & 200 & 1.3 & 1.1 & 2.4 & 7 \\
\hline WNI2 & 170 & 0 & 160 & 80 & 40 & 120 & 2.9 & 0.9 & 3.9 & 5 \\
\hline SENI2 & 40 & 0 & 280 & 130 & -40 & 40 & 6.4 & 6.6 & 13.0 & 57 \\
\hline
\end{tabular}

poor and the models cannot match the largest delay time.

The poor fit of the two-layer model with dipping symmetry axis is stressed by the $95 \%$ confidence regions (figure G.6). The plots show the projection of the six-dimensional parameter space (fast polarisation, delay time and path length for the two layers) onto a two dimensional plane. The crosses mark models that lie within the $95 \%$ error region to the bestfit solution. The abundance of models in the $95 \%$ region illustrates that the best-fit model is poorly constrained. 


\section{APPENDIX H}

\section{Travel time residuals}

\section{H.1 Travel time residuals for fast and slow components}

As mentioned in chapter 9 , attempts were made to examine travel time residuals on the fast and slow component of the data. Two different methods are applied, both of which have some drawbacks. The fast polarisation varies slightly between stations and with incoming polarisation (i.e. backazimuth). This implies a separate rotation for each event. However, in this case travel time residuals can only be estimated for recordings that gave reliable splitting parameters in the shear-wave splitting analysis. Not every cross-correlation then delivers good results, so that the amount of measurements for this method is decimated largely (figure H.1). To surpass this problem, another method rotates all recording in the same, average fast polarisation. Two different average fast polarisations of $17^{\circ}$ and $45^{\circ}$ are chosen (figure H.2). This method allows an extended dataset to be analysed, but for individual measurements the average fast polarisation might deviate strongly from the real fast polarisation for the particular measurement.

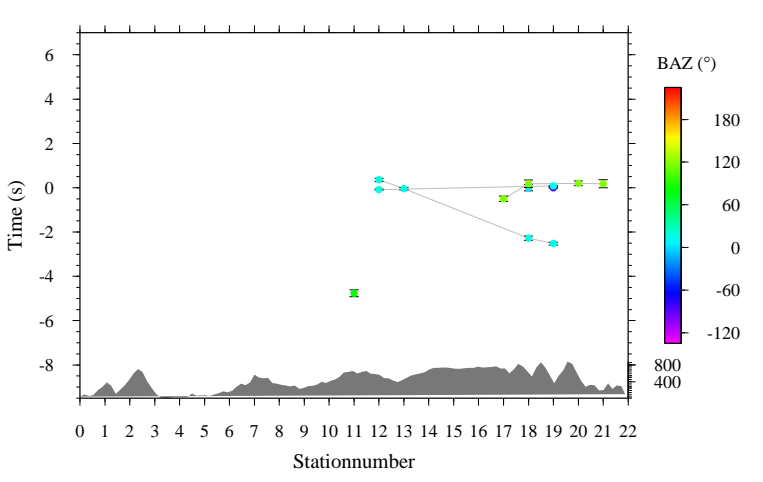

(a) Fast

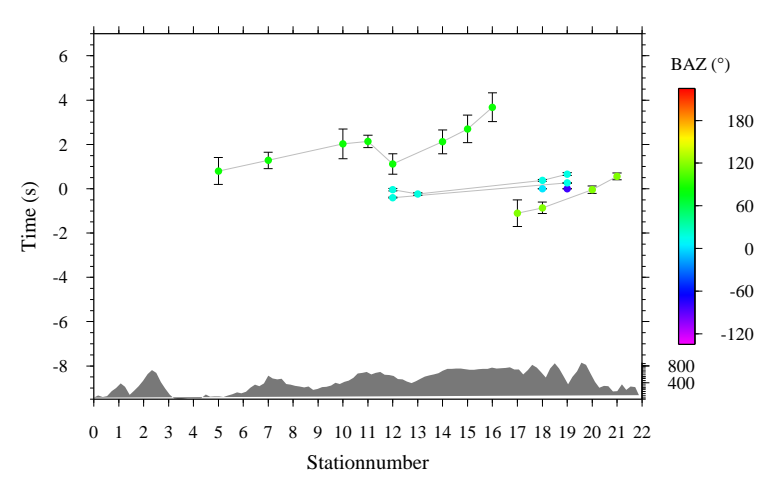

(b) Slow

FIGURE H.1 Travel time residuals on traces rotated into individual fast and slow polarisation 


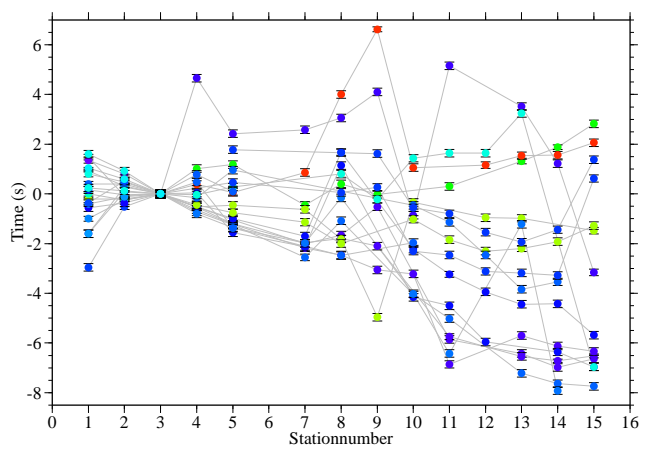

(a) Fast, $\Phi=17^{\circ}$

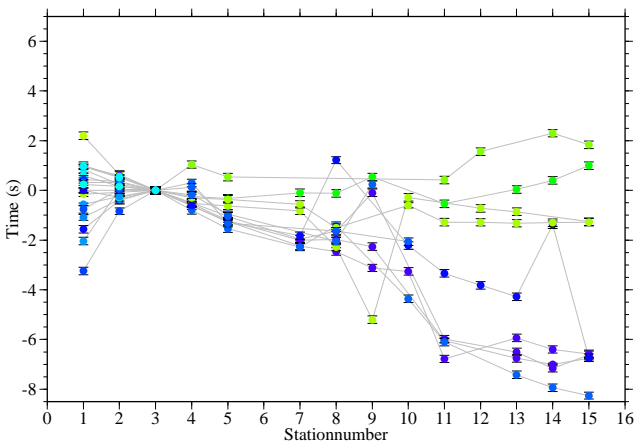

(c) Fast, $\Phi=45^{\circ}$

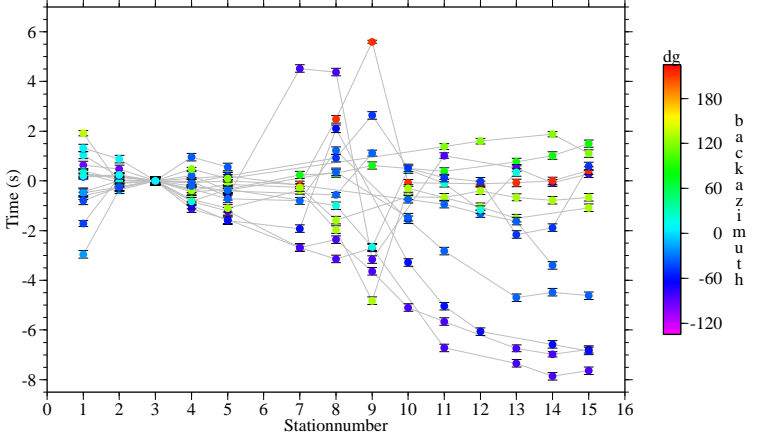

(b) Slow, $\Phi=107^{\circ}$

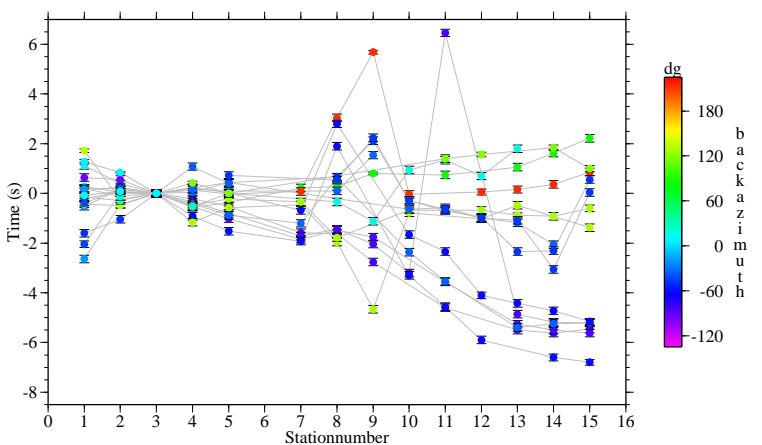

(d) Slow, $\Phi=135^{\circ}$

FIGURE H.2 Travel time residuals on traces rotated into two different sets of average fast and slow polarisations

\section{H.2 Travel time residuals due to crustal thickness variations}

Assuming a thinned crustal thickness of $h_{\min }$ and a thicker crustal thickness of $h_{\max }$, with a crustal velocity of $v_{c}$ and a mantle velocity of $v_{m}$ with $v_{c}<v_{m}$ the difference in travel time for the path trough the thicker and the thinner crust can be estimated as:

$$
\begin{aligned}
\Delta t & =\frac{h_{\max }}{v_{c} \cdot \cos i}-\left(\frac{h_{\min }}{v_{c} \cdot \cos i}+\frac{h_{\max }-h \min }{v_{m} \cdot \cos i}\right) \\
& =\frac{h_{\max }-h_{\min }}{v_{c} \cdot \cos i}-\frac{h_{\max }-h_{\min }}{v_{m} \cdot \cos i} \\
& =\frac{\Delta h}{\cos i}\left(\frac{1}{v_{c}}-\frac{1}{v_{m}}\right),
\end{aligned}
$$

where $i$ is the incidence angle measured from the vertical. 


\section{References}

Abramson, E. H., SlutSKy, L. J., BROWN, J. M., AND ZAUG, J. 1997. The elastic constants of San Carlos olivine to 17 GPa. Journal of Geophysical Research B: Solid Earth, 102(6), 1225312263.

ADAMS, B., WRIGHT, S., AND KUnZE, K. 1993. Orientation mapping: The emergence of a new microscopy. Metallurgical Transactions, A 24, 819-831.

ADAMS, R., AND FERRIS, B. 1976. A further earthquake at exceptional depth beneath New Zealand. New Zealand Journal of Geology and Geophysics, 19(2), 269-273.

Aharonov, E., Whitehead, J. A., Kelemen, P. B., And Spiegelman, M. 1995. Channeling instability of upwelling melt in the mantle. Journal of Geophysical Research, 100(B10), 20,433-20,450.

AlsinA, D., AND SNIEDER, R. 1995. Small-scale sublithosperic continental mantle deformation: constraints from SKS splitting observations. Geophysical Journal International, 123, 431-448.

Anderson, D. 1989. Theory of the Earth. Blackwell Scientific Publications.

Anderson, H., And WebB, T. 1994. New Zealand seismicity: Pattern revealed by the upgraded National Seismograph Network. New Zealand Journal of Geology and Geophysics, 37, 477-493.

Anderson, M., ZAndt, G., Triep, E., FOUCh, M., AND BeCK, S. 2004. Anisotropy and mantle flow in the Chile-Argentina subduction zone from shear wave splitting analysis. Geophysical Research Letters, 31(23), 1-4.

ANDERSON, O., AND ISAAK, D. 1995. Elastic constants of mantle minerals at high temperatures. Pages 64-97 of: AHRens, T. (ed), Mineral Physics and Crystallography: A handbook of physical constants, vol. 2. Washington, D.C.: AGU Reference Shelf.

ANDO, M., ISHIKAWA, Y., AND YAMAZAKI, F. 1983. Shear wave polarization anisotropy in the upper mantle beneath Honshu, Japan. Journal of Geophysical Research, 88, 5850-5864.

AnGlin, D. K., AND Fouch, M. J. 2005. Seismic anisotropy in the Izu-Bonin subduction system. Geophysical Research Letters, 32(9b), 1-4.

ANSELL, J., AND BANNISTER, S. 1996. Shallow morphology of the subducted Pacific plate along the Hikurangi margin, New Zealand. Physics of the Earth and Planetary Interiors, 93, $3-20$.

Arcay, D., Doin, M.-P., TRIC, E., Bousquet, R., AND DE CAPITANI, C. 2006. Overriding plate thinning in subduction zones: Localized convection induced by slab dehydration. Geochemistry Geophysics Geosystems, 7(doi:10.1029/2005GC001061), 1-26. 
Asimow, P., Hirschmann, M., Ghiorso, M., O'HARA, M., And Stolper, E. 1995. The effect of pressure-induced solid-solid phase transitions on decompression melting of the mantle. Geochimica et Cosmochimica Acta, 59(21), 4489-4506.

Asimow, P., HirschmanN, M., AND StOlPER, E. 2001. Calculation of peridotite partial melting from thermodynamic models of minerals and melts, IV. Adiabatic decompression and the composition and mean properties of mid-ocean ridge basalts. Journal of Petrology, 42(5), 963-998.

ASU. Seismic Anisotropy Database. http://geophysics.asu.edu/anisotropy/.

Audoine, E., Savage, M. K., AND Gledhill, K. 2000. Seismic anisotropy from local earthquakes in the transition region from a subduction to a strike-slip plate boundary, New Zealand. Journal of Geophysical Research, 105(B4), 8013-8033.

Audoine, E., SAVAGe, M. K., AND Gledhill, K. R. 2004. Anisotropic structure under a back-arc spreading region, the Taupo Volcanic Zone, New Zealand. Journal of Geophysical Research, 109(B11), 10.1029/2003JB002932.

Ayele, A., Stuart, G., And Kendall, J. M. 2004. Insights into rifting from shear wave splitting and receiver functions: An example from Ethiopia. Geophysical Journal International, 157(1), 354-362.

BABUŠKA, V. 1972. Elasticity and Anisotropy of Dunite and Bronzitite. Journal of Geophysical Research, 77(35), 6955-6965.

BABUŠKA, V., AND CARA, M. 1991. Seismic anisotropy in the Earth. Modern Approaches in Geophysics, vol. 10. Dordrecht: Kluwer Academic Publishers.

BabušKa, V., PlomerovÁ, J., Vecsey, L., Granet, M., And Achauer, U. 2002. Seismic anisotropy of the French Massif Central and predisposition of Cenozoic rifting and volcanism by Variscan suture hidden in the mantle lithosphere. Tectonics, 21(4), 11-1.

BACKUS, G. E. 1962. Long-wave elastic anisotropy produced by horizontal layering. Journal of Geophysical Research, 67(11), 4427-4440.

Bai, Q., Mackwell, S. J., AND Kohlstedt, D. L. 1991. High-Temperature Creep of Olivine Single Crystals 1. Mechanical Results for Buffered Samples. Journal of Geophysical Research, 96(B2), 2441-2463.

BANNISTER, S., BRYAN, C., AND BIBBY, H. 2004. Shear wave velocity variation across the Taupo Volcanic Zone, New Zealand, from receiver function inversion. Geophysical Journal International, 159, 291-310.

BAnnister, S., Reyners, M., Stuart, G., AND SAVAGe, M. 2007. Imaging the Hikurangi subduction zone, New Zealand, using teleseismic receiver functions: crustal fluids above the forearc mantle wedge. Geophysical Journal International, 169(2), 602-616.

BARRUOL, G., AND Kern, H. 1996. P and S waves velocities and shear wave splitting in the lower crustal/upper mantle transition (Ivrea Zone). Experimental and calculated data. Physics of the Earth and Planetary Interior, 95, 175-194.

BARRUOL, G., AND MAINPRICE, D. 1993. A quantitative evaluation of the contribution of crustal rocks to the shear-wave splitting of teleseismic SKS waves. Physics of the Earth and Planetary Interiors, 78(3-4), 281-300. 
BARTON, N. 2007. Rock quality, seismic velocity, attenuation and anisotropy. Taylor \& Francis/Balkema.

Bascou, J., Barruol, G., Vauchez, A., Mainprice, D., And Egydio-Silva, M. 2001. EBSD-measured lattice-preferred orientations and seismic properties of eclogites. Tectonophysics, 342(1-2), 61-80.

Bastow, I. D., Stuart, G. W., Kendall, J. M., AND Ebinger, C. J. 2005. Upper-mantle seismic structure in a region of incipient continental breakup: Northern Ethiopian rift. Geophysical Journal International, 162(2), 479-493.

BEAVEN, J., AND HAines, J. 2001. Contemporary horizontal velocity and strain rate fields of the Pacific-Australien plate boundary zone through New Zealand. Journal of Geophysical Research, 106(B1), 741-770.

Bebout, G. E. 2007. Metamorphic chemical geodynamics of subduction zones. Earth and Planetary Science Letters, 260(3-4), 373-393.

Becker, T. W., KellogG, J. B., EKström, G., And O'Connell, R. J. 2003. Comparison of azimuthal seismic anisotropy from surface waves and finite strain from global mantlecirculation models. Geophysical Journal International, 155(2), 696-714.

Behn, M. D., Silver, P. G., AND Conrad, C. P. 2004. Detection of upper mantle flow associated with the African Superplume. Earth and Planetary Science Letters, 224(3-4), 259274.

Behn, M. D., HiRTh, G., AND Kelemen, P. B. 2007. Trench-parallel anisotropy produced by foundering of arc lower crust. Science, 317, 108-111.

Ben-IsmaïL, W., BARruol, G., AND MAinPrice, D. 2001. The Kaapvaal craton seismic anisotropy: Petrophysical analyses of upper mantle kimberlite nodules. Geophysical Research Letters, 28(13), 2497-2500.

Best, A. I., SothCotT, J., AND MCCANN, C. 2007. A laboratory study of seismic velocity and attenuation anisotropy in near-surface sedimentary rocks. Geophysical Prospecting, 55(5), 609-625.

BILlen, M. I. 2008. Modeling the Dynamics of Subducting Slabs. Annual Review of Earth and Planetary Sciences, 36(1), 325-356.

BlackMAn, D. K., AND Kendall, J. M. 1997. Sensitivity of teleseismic body waves to mineral texture and melt in the mantle benaeth a mid-ocean ridge. Philosophical Transactions of the Royal Society of London, 355, 217-231.

Blackman, D., Kendall, J.-M., DAWson, P., Wenk, H.-R., BOYCE, D., AND Morgan, J. 1996. Teleseismic imaging of subaxial flow at mid-ocean ridges: Traveltime effects of anisotropic mineral texture in the mantle. Geophysical Journal International, 127(2), 415-426.

Bock, G., Kind, R., Rudloff, A., AND Asch, G. 1998. Shear wave anisotropy in the upper mantle beneath the Nazca plate in northern Chile. Journal of Geophysical Research B: Solid Earth, 103(10), 24333-24345.

BokelmanN, G. H. R., AND Silver, P. G. 2000. Mantle Variation within the Canadian Shield: Travel Times from the APT89 Portable Broadband Transect. Journal of Geophysical Research, 105, 579-605. 
Bolfan-Casanova, N. 2005. Water in the Earth's mantle. Mineralogical Magazine, 69(3), 229-257.

BOlton, H., AND MASTERS, G. 2001. Travel times of P and S from the global digital seismic networks: Implications for the relative variation of $\mathrm{P}$ and $\mathrm{S}$ velocity in the mantle. Journal of Geophysical Research, 106(B7), 13,527-13,540.

BOYD, O. S. 2006. An efficient Matlab script to calculate heterogeneous anisotropically elastic wave propagation in three dimensions. Computers $\mathcal{E}$ Geosciences, 32(2), 259-264.

Braun, M. G., And Kelemen, P. B. 2002. Dunite distribution in the Oman Ophiolite: Implications for melt flux through porous dunite conduits. Geochemistry Geophysics Geosystems, 3(11), 1-21.

BRAUn, M. G., AND SOHN, R. A. 2003. Melt migration in plume-ridge systems. Earth and Planetary Science Letters, 213(3-4), 417-430.

BRIGGS, R. M. 1986. Volcanic rocks of the Waikato region, western North Island, and some petrological and tectonic constraints on their origin. Pages 76-91 of: SMITH, I. E. M. (ed), Late Cenozoic Volcanism in New Zealand. Wellington: R. Soc. of New Zealand.

Brisbourne, A., Stuart, G., And Kendall, J. M. 1999. Anisotropic structure of the Hikurangi subduction zone, New Zealand-integrated interpretation of surface-wave and body-wave observations. Geophysical Journal International, 137(1), 214-230.

Brown, G., HAWKesworth, C., AND Wilson, C. (eds). 1992. Understanding the Earth. Cambridge University Press.

BUCK, W. R. 2004. Consequences of asthenospheric variability on continental rifting. Vol. Rheology and deforamtion of the lithosphere at continental margins. Columbia University Press. Chap. 1, pages 1-30.

ButTLES, J., AND Olson, P. 1998. A laboratory model of subduction zone anisotropy. Earth and Planetary Science Letters, 164(1-2), 245-262.

Cammarano, F., Goes, S., VACher, P., AND Giardini, D. 2003. Inferring upper-mantle temperatures from seismic velocities. Physics of the Earth and Planetary Interiors, 138(3-4), 197-222.

CANDE, S., AND STOCK, J. 2004. Pacific-Antarctic-Australia motion and the formation of the Macquarie Plate. Geophysical Journal International, 157, 399-414.

Carcione, J. J. M. (ed). 2007. Wave Fields in Real Media: Wave Propagation in Anisotropic, Anelastic, Porous and Electromagnetic Media. Elsevier Science; 2 edition.

Chai, M., BRown, J. M., AND SlutSKy, L. J. 1997. The elastic constants of an aluminous orthopyroxene to 12.5 GPa. Journal of Geophysical Research B: Solid Earth, 102(B7), 14779_ 14785.

CHAPMAN, M. 2003. Frequency-dependent anisotropy due to meso-scale fractures in the presence of equant porosity. Geophysical Prospecting, 51, 369-379.

ChAPMAN, M., ZATSEPIN, S., AND CRAMPIN, S. 2002. Derivation of a microstructural poroelastic model. Geophysical Journal International, 151(2), 427-451.

Chapman, M., Maultzsch, S., LiU, E., AND Li, X. Y. 2003. The effect of fluid saturation in an anisotropic multi-scale equant porosity model. Journal of Applied Geophysics, 54(3-4), 191-202. 
Chen, W. P., And ÖZAlaybey, S. 1998. Correlation between seismic anisotropy and Bouguer gravity anomalies in Tibet and its implications for lithospheric structures. Geophysical Journal International, 135(1), 93-101.

Chevrot, S., Favier, N., And Komatitsch, D. 2004. Shear wave splitting in threedimensional anisotropic media. Geophysical Journal International, 159(2), 711-720.

Christensen, N. 2004. Serpentinites, peridotites, and seismology. International Geology Review, 46, 795-816.

COCHRAN, E. 1999. SKS shear-wave splitting observations in the mantle beneath East Cape, New Zealand. Report. University of Leeds.

COLE, J. 1990. Structural control and origin of volcanism in the Taupo volcanic zone, New Zealand. Bulletin of Volcanology, 52(6), 445-459.

Collins, M. D., AND BRown, J. M. 1998. Elasticity of an upper mantle clinopyroxene. Physics and Chemistry of Minerals, 26(1), 7-13.

Connolly, J. 2005. Computation of phase equilibria by linear programming: A tool for geodynamic modeling and its application to subduction zone decarbonation. Earth and Planetary Science Letters, 236(1-2), 524-541.

Conrad, C. P., Behn, M. D., AND Silver, P. G. 2007. Global mantle flow and the development of seismic anisotropy: Differences between the oceanic and continental upper mantle. Journal of Geophysical Research, 112(B07317, doi:10.1029/2006JB004608).

CONRAD, C., AND Lithgow-BERTELlOni, C. 2002. How mantle slabs drive plate tectonics. Science, 298(5591), 207-209.

Cornwell, D., Maguire, P., Kendall, J. M., England, R., Mackenzie, G., AND GROUP, E. W. 2004. Travel-time residuals and shear-wave splitting across the main Ethiopian Rift. Page 47 of: The East African Rift System: Geodynamics, Resources and Environment, Addis Ababa.

CRAMPIN, S. 1993. A review of the effects of crack geometry on wave propagation through aligned cracks. Canadian Journal of Exploration Geophysics, 29(1), 3-17.

CRAMPIN, S. 1994. The fracture criticality of crustal rocks. Geophysical Journal International, 118, $428-438$.

CRAMPIN, S., AND CHASTIN, S. 2003. A review of shear wave splitting in the crack-critical crust. Geophysical Journal International, 155, 221-240.

CRAMPIN, S. 1984. An introduction to wave propagation in anisotropic media. Geophysical Journal International, 76(1), 17-28.

Currie, C. A., CAssidy, J. F., Hyndman, R. D., And Bostock, M. G. 2004. Shear wave anisotropy beneath the Cascadia subduction zone and western North America craton. Geophysical Journal International, 157(1), 341-353.

DAines, M. J., AND KohlstedT, D. 1997. Influence of deformation on melt topology in peridotites. Journal of Geophysical Research, 102(B5), 10,257-10,271.

DARBY, D. J., AND MEERTENS, C. M. 1995. Terrestrial and GPS measurements of deformation across the Taupo back arc and Hikurangi forearc regions in New Zealand. Journal of Geophysical Research, 100(B5), 8221-8232. 
DAubeCHIES, I. 1992. Ten Lectures on Wavelets (CBMS-NSF Regional Conference Series in Applied Mathematics). Society for Industrial and Applied Mathematics.

DAVIES, G. 1999. Dynamic Earth. Cambridge University Press, New York.

Davies, J. H., And Stevenson, D. J. 1992. Physical Model of Source Region of Subduction Zone Volcanics. Journal of Geophysical Research, 97(B2), 2037-2070.

DAVIES, J. C. 1986. Statistics and data analysis in geology. Jon Wiley and Sons, Inc.

DAVY, B., AND WOOD, R. 1994. Gravity and magnetic modelling of the Hikurangi Plateau. Marine Geology, 118(1-2), 139-151.

Demets, C., Gordon, R., Argus, D., and Stein, S. 1990. Current plate motions. Geophysical Journal International, 101(2), 425-478.

Dewandel, B., Boudier, F., Kern, H., Warsi, W., And Mainprice, D. 2003. Seismic wave velocity and anisotropy of serpentinized peridotite in the Oman ophiolite. Tectonophysics, 370(1-4), 77-94.

DingWelL, D. 2007. Properties of rock and minerals - Diffusion, viscosity, and flow of melt. In: Gerald Schubert, G. D. P. (ed), Treatise on Geophysics, vol. 2, Mineral Physics. Elsevier.

Doglioni, C., Carminati, E., Cuffaro, M., And Scrocca, D. 2007. Subduction kinematics and dynamic constraints. Earth-Science Reviews, 83(3-4), 125-175.

DUCLOS, M. 2005. Insights on the plate boundary deformation from seismic anisotropy in the New Zealand upper mantle. PhD thesis, Victoria University of Wellington.

Duclos, M., Savage, M., Tommasi, A., And Gledhill, K. 2005. Mantle tectonics beneath New Zealand inferred from SKS splitting an petrophysics. Geophysical Journal International, 162, 1-15.

DZIEWONSKI, A., AND ANDERSON, D. 1981. Preliminary reference Earth model. Physics of the Earth and Planetary Interiors, 25(4), 297-356.

Eberhart-Phillips, D., AND Henderson, M. C. 2004. Including anisotropy in 3-D velocity inversion and application to Marlborough, New Zealand. Geophysical Journal International, 156(2), 237-254.

Eberhart-Phillips, D., Reyners, M., Chadwick, M., And ChiU, J.-M. 2005. Crustal heterogeneity and subduction processes: 3-D vp, vp/vs and $\mathrm{Q}$ in the southern North Island, New Zealand. Geophysical Journal International, 162, 270-288.

ELKINS-TANTON, L., AND GROVE, T. 2003. Evidence for deep melting of hydrous metasomatized mantle: Pliocene high-potassiuim magmas from the Sierra Nevadas. Journal of Geophysical Research B: Solid Earth, 108(7), 1-19.

ENGDAhL, E. R., AND GubBins, D. 1987. Simultaneous Travel Time Inversion for Earthquake Location and Subduction Zone Structure in the Central Aleutian Islands. Journal of Geophysical Research, 92(B13), 13,855-13,862.

EngDahl, E., AND RitzWoller, M. 2001. Crust and upper mantle P- and S-wave delay times at Eurasian seismic stations. Physics of the Earth and Planetary Interiors, 123, 205-219. 
EsHelBy, J. 1957. The determination of the elastic field of an ellipsoidal inclusion, and related problems. Proceedings of the Royal Society of London. Series A, Mathematical and Physical Sciences, 241(1226), 376-396.

Estey, L. H., AND Douglas, B. J. 1986. Upper mantle anisotropy: a preliminary model. Journal of Geophysical Research, 91(B11), 11393-11406.

FACCENDA, M., Burlini, L., GerYA, T. V., AND MAINPRICE, D. 2008. Fault-induced seismic anisotropy by hydration in subducting oceanic plates. Nature, 455(7216), 1097-1100.

FAul, U. H., Fitz Gerald, J. D., AND JACKSON, I. 2004. Shear wave attenuation and dispersion in melt-bearing olivine polycrystals: 2. Microstructural interpretation and seismological implications. Journal of Geophysical Research, 109, B06202, doi:10.1029/2003JB002407.

FAUL, U. 1997. Permeability of partially molten upper mantle rocks from experiments and percolation theory. Journal of Geophysical Research B: Solid Earth, 102(B5), 10299-10311.

FAUL, U., TOOMEY, D., AND WAFF, H. 1994. Intergranular basaltic melt is distributed in thin, elongated inclusions. Geophysical Research Letters, 21, 29-32.

Fischer, K. M., AND WiENS, D. A. 1996. The depth distribution of mantle anisotropy beneath the Tonga subduction zone. Earth and Planetary Science Letters, 142(1-2), 253-260.

FischeR, K., AND YANG, X. 1994. Anisotropy in Kuril-Kamchatka subduction zone structure. Geophysical Research Letters, 21(1), 5-8.

Fischer, K., Fouch, M., Wiens, D., And Boettcher, M. 1998. Anisotropy and flow in Pacific subduction zone back-arcs. Pure and Applied Geophysics, 151, 463-475.

Fischer, K., LI, A., FORSYTH, D., AND HUNG, S.-H. 2005. Imaging three-dimensional anisotropy with broadband seismometer arrays. Pages 99-116 of: LEVANDER, A., AND NOLET, G. (eds), Seismic Earth: Array analysis of broadband seismograms. AGU Monograph, vol. 157.

Forsyth, J., Smith Lyttle, B., AND Morrison, B. 1972. Geological Map of New Zealand. Tech. rept. Institute of Geological \& Nuclear Sciences (GNS).

FOUCH, M. J., AND Fischer, K. M. 1996. Mantle anisotropy beneath northwest Pacific subduction zones. Journal of Geophysical Research B: Solid Earth, 101(7), 15987-16002.

FOUCH, M. J., AND FISCHER, K. M. 1998. Shear wave anisotropy in the Mariana subduction zone. Geophysical Research Letters, 25(8), 1221-1224.

Fouch, M. J., JAmes, D. E., VAnDeCAR, J. C., AND VAn Der Lee, S. 2004. Mantle seismic structure beneath the Kaapvaal and Zimbabwe Cratons. South African Journal of Geology, 107(1-2), 33-44.

Fowler, C. M. R. 2001. The solid earth. Cambridge University Press.

Funiciello, F., Moroni, M., Piromallo, C., Faccenna, C., Cenedese, A., And BUI, H. 2006. Mapping mantle flow during retreating subduction: Laboratory models analyzed by feature tracking. Journal of Geophysical Research, 111(B03402, doi:10.1029/2005JB003792).

Funnell, R., Chapman, D., Allis, R., And Armstrong, P. 1996. Thermal state of Taranaki Basin, New Zealand. Journal of Geophysical Research, 101, 25,197-25,215. 
FURUKAWA, Y. 1993. Magmatic processes under arcs and formation of the volcanic front. Journal of Geophysical Research, 98, 830 - 8319.

Gaboret, C., Forte, A. M., And Montagner, J. P. 2003. The unique dynamics of the Pacific hemisphere mantle and its signature on seismic anisotropy. Earth and Planetary Science Letters, 208(3-4), 219-233.

Gaetani, G. A., AND GROVE, T. L. 1998. The influence of water on melting of mantle peridotite. Contributions to Mineralogy and Petrology, 131(4), 323-346.

GALEA, P. 1993. Upper mantle anisotropy in the SW Pacific from earthquake travel-time analysis. Physics of the Earth and Planetary Interiors, 76(3-4), 229-239.

GeONet. 2001. The GeoNet Project - Monitoring geological hazards in New Zealand. Website: http://www.geonet.org.nz.

Gerya, T., Yuen, D., AND SEVRE, E. 2004. Dynamical causes for incipient magma chambers above slabs. Geology, 32, 89-92.

GILL, J. B. 1981. Orogenic andesites and plate tectonics. Springer Verlag.

GLedHILL, K., AND GubBINS, D. 1996. SKS splitting and the seismic anisotropy of the mantle beneath the Hikurangi subduction zone, New Zealand. Physics of the Earth and Planetary Interiors, 95, 227-236.

Gledhill, K. R., And StUART, G. 1996. Seismic anisotropy in the fore-arc region of the Hikurangi subduction zone, New Zealand. Physics of the Earth and Planetary Interiors, 95, 211-225.

GLEDHILL, K. 1991. Evidence for shallow and pervasive seismic anisotropy in the Wellington region, New Zealand. Journal of Geophysical Research, 96(B13), 21,503-21,516.

GÖK, R., Ni, J. F., West, M., SAndVOl, E., Wilson, D., Aster, R., BAldRidGe, W. S., Grand, S., GaO, W., Tillmann, F., And SEmken, S. 2003. Shear wave splitting and mantle flow beneath LA RISTRA. Geophysical Research Letters, 30(12), 16-1.

GrechKA, V. 2005. Penny-shaped fractures revisited. Studia Geophysica et Geodaetica, 49(3), 365-381.

GREVE, S., AND SAVAGE, M. 2007 (4-7 June). Modeling seismic anisotropy acorss a subduction zone: Central North Island, New Zealand. In: Subduction zone geodynamics conference, vol. 41.

Greve, S., SAVAGE, M., AND HofmAnN, S. 2008. Strong variations in seismic anisotropy across the Hikurangi subduction zone, North Island, New Zealand. Tectonophysics, 462(14), 7-21.

Grove, T. L., Chatterjee, N., Parman, S. W., And Medard, E. 2006. The influence of $\mathrm{H} 2 \mathrm{O}$ on mantle wedge melting. Earth and Planetary Science Letters, 249(1-2), 74-89.

Gueguen, Y., AND Poirier, A. 1980. Deformation of mantle rocks. Annual Review of Earth and Planetary Sciences, 2(8), 119-144.

Guilbert, J., Poupinet, G., AND MeI, J. 1996. A study of azimuthal P residuals and shearwave splitting across the Kunlun range (Northern Tibetian plateau). Physics of the Earth and Planetary Interiors, 95, 167-174. 
HAGER, B., AND O'CONNELL, R. 1981. A simple global model of plate dynamics and mantle convection. Journal of Geophysical Research, 86(B6), 4843-4867.

HAINES, A. J. 1979. Seismic wave velocities in the uppermost mantle beneath New Zealand. New Zealand Journal of Geology and Geophysics, 22(2), 245-257.

Hall, C. E., Fischer, K. M., Parmentier, E. M., and Blackman, D. K. 2000. The influence of plate motions on three-dimensional back arc mantle flow and shear wave splitting. Journal of Geophysical Research B: Solid Earth, 105(B12), 28009-28033.

HAMMOND, W., AND HuMPHREYS, E. 2000. Upper mantle seismic wave attenuation: Effects of realistic partial melt distribution. Journal of Geophysical Research, 105(B5), 10,98710,999 .

HARRISON, A., AND WHITE, R. 2004. Crustal structure of the Taupo Volcanic Zone, New Zealand: Stretching and igneous intrusion. Geophysical Research Letters, 31(L13615), doi:10.1029/2004GL019885.

HARRISON, A., AND WHITE, R. S. 2006. Lithospheric structure of an active backarc basin: The Taupo Volcanic Zone, New Zealand. Geophysical Journal International, 167(2), 968-990.

HARTOG, R., AND SCHWARTZ, S. Y. 2000. Subduction-induced strain in the upper mantle east of the Mendocino triple junction, California. Journal of Geophysical Research B: Solid Earth, 105(B4), 7909-7930.

HATHERTON, T. 1970. Upper mantle inhomogenity beneath New Zealand: surface manifestations. Journal of Geophysical Research, 75, $269-284$.

Hawkesworth, C. J., Turner, S. P., McDermott, F., Peate, D. W., and van CalSTEREN, P. 1997. U-Th Isotopes in Arc Magmas: Implications for Element Transfer from the Subducted Crust. Science, 276(5312), 551-555.

Heintz, M., And KennetT, B. L. N. 2005. Continental scale shear wave splitting analysis: Investigation of seismic anisotropy underneath the Australian continent. Earth and Planetary Science Letters, 236(1-2), 106-119.

HelBiG, K., AND THOMSEN, L. 2005. 75-plus years of anisotropy in exploration and reservoir seismics: A historical review of concepts and methods. Geophysics, 70(6), 9ND-23ND.

HeNrys, S. A., AND THE NIGHT WORKING GROUP. 2001. The North Island Geophysical Transect; an overview. N.Z, Geophysical Society Annual Symposium, Wairakei.

Henrys, S., Reyners, M., Pecher, I., Bannister, S., Nishimura, Y., And Maslen, G. 2006. Kinking of the subducting slab by escalator normal faulting beneath the North Island of New Zealand. Geology, 34(9), 777-780.

HESS, H. 1964. Seismic anisotropy of the uppermost mantle under oceans. Nature, 203, 629-631.

HiEsS, J., COLE, J. W., AND SPINKS, K. 2007. Influence of the crust and crustal structure on the location and composition of high-alumina basalts of the Taupo Volcanic Zone, New Zealand. New Zealand Journal of Geology and Geophysics, 50, 327-342.

Higdon, R. 1991. Absorbing boundary conditions for elastic waves. Geophysics, 56(2), 231-241.

HiLl, R. 1952. The elastic behavior of crystalline aggregates. Philosophical Transactions of the Royal Society of London, A65, 349-354. 
Hiramatsu, Y., Ando, M., TsukudA, T., AND OoidA, T. 1998. Three-dimensional image of the anisotropic bodies beneath central Honshu, Japan. Geophysical Journal International, 135(3), 801-816.

Hirschmann, M. M., Aubaud, C., And Withers, A. C. 2005. Storage capacity of H2O in nominally anhydrous minerals in the upper mantle. Earth and Planetary Science Letters, 236(1-2), 167-181.

HirschmanN, M., Asimow, P., Ghiorso, M., AND StOlPer, E. 1999. Calculation of Peridotite Partial Melting from Thermodynamic Models of Minerals and Melts. III. Controls on Isobaric Melt Production and the Effect of Water on Melt Production. Journal of Petrology, 40, 831-851.

HiRTH, G., AND KOHLSTEDT, D. 1996. Water in the oceanic upper mantle: Implications for rheology, melt extraction and the evolution of the lithosphere. Earth and Planetary Science Letters, 144(1-2), 93-108.

HofmanN, S. D. 2003. Seismic anisotropy in the crust and mantle: A study at the western edge of the Central Volcanic Region, New Zealand. Master's Thesis, Victoria University of Wellington, Wellington.

Holtzman, B. K., AND KendALl, J.-M. 2008. Organized melt, seismic anisotropy and plate boundary dynamics. in preparation.

Holtzman, B. K., Kohlstedt, D. L., Zimmerman, M. E., Hiraga, T., Hustoft, J., AND HeIDELBACH, F. 2003a. Melt segregation and strain partitioning: Implications for seismic anisotropy and mantle flow. Science, 301(5637), 1227-1230.

Holtzman, B. K., Kohlsted, D. L., And MorgAn, J. P. 2005. Viscous energy dissipation and strain partitioning in partially molten rocks. Journal of Petrology, 46(12), 2569-2592.

Holtzman, B. K., AND Kohlstedt, D. L. 2007. Stress-driven Melt Segregation and Strain Partitioning in Partially Molten Rocks: Effects of Stress and Strain. Journal of Petrology, 48(12), 2379-2406.

Holtzman, B., Groebner, N., Zimmerman, M., Ginsberg, S., And Kohlstedt, D. 2003b. Stress-driven melt segregation in partially molten rocks. Geochemistry Geophysics Geosystems, 4(5), 8607, doi:10.1029/2001GC000258.

Horspool, N. A., SAVAGE, M. K., AND BANnister, S. 2006. Implications for intraplate volcanism and back-arc deformation in northwestern New Zealand, from joint inversion of receiver functions and surface waves. Geophysical Journal International, 166(3), 14661483.

Hudson, J. A. 1980. Overall properties of a cracked solid. Mathematical Proiceedings of the Cambridge Philosophical Society, 88, 371-384.

HUDSON, J. 1981. Wave speeds and attenuation of elastic waves in material containing cracks. Geophysical Journal of the Royal Astronomical Society, 64, 133-150.

IRIS CONSORTIUM, U. S. G. S. 1993 (February). SEED - Standard for the exchange of earthquake data, Reference manual, SEED form version 2.3. Federation of digital seismographic network incorporated research institutions for seismology.

IsAAK, D. G., ANDERSON, O. L., AND COHEN, R. E. 1992. The relationship between shear and compressional velocities at high pressures: Reconciliation of seismic tomography and mineral physics. Geophysical Research Letters, 19(8), 741-744. 
ISAAK, D. 2001. Handbook of Elastic Properties of Solids, Liquids, and Gases. Vol. Volume III: Elastic Properties of Solids: Biological and Organic Materials, Earth and Marine Sciences. Academic Press. Chap. Elastic properties of minerals and planetary objects, pages 325376.

ISC. Automated Splitting Project. http://www.isc.ac.uk/SKS/index2.html.

ISMAÏL, W. B., AND MAINPRICE, D. 1998. An olivine fabric database: An overview of upper mantle fabrics and seismic anisotropy. Tectonophysics, 296(1-2), 145-157.

IWAMORI, H. 1998. Transportation of $\mathrm{H} 2 \mathrm{O}$ and melting in subduction zones. Earth and Planetary Science Letters, 160(1-2), 65-80.

JaCKSON, I., Fitz Gerald, J., FAul, U., AND TAN, B. 2002. Grain-size-sensitive seismic wave attenuation in polycrystalline olivine. Journal of Geophysical Research B: Solid Earth, 107(12), 1-16.

JACOBSEN, S. D. 2006. Effect of water on the equation of state of nominally anhydrous minerals. Reviews in Mineralogy and Geochemistry, 62(1), 321-342.

Jaeger, J. C., CoOK, N. G. W., And Zimmerman, R. W. 2007. Fundamentals of Rock Mechanics. Blackwell.

Jagoutz, O., Muntener, O., BurG, J.-P., Ulmer, P., And Jagoutz, E. 2006. Lower continental crust formation through focused flow in $\mathrm{km}$-scale melt conduits: The zoned ultramafic bodies of the Chilas Complex in the Kohistan island arc (NW Pakistan). Earth and Planetary Science Letters, 242(3-4), 320-342.

James, D. E., Fouch, M. J., VanDecar, J. C., Van Der Lee, S., and KaApVal. 2001. Tectospheric structure beneath southern Africa. Geophysical Research Letters, 28(13), 24852488.

Jousselin, D., AND MAINPRICE, D. 1998. Melt topology and seismic anisotropy in mantle peridotites of the Oman ophiolite. Earth and Planetary Science Letters, 164(3-4), 553-568.

JUNG, H., AND KARATO, S. I. 2001a. Effects of water on dynamically recrystallized grainsize of olivine. Journal of Structural Geology, 23(9), 1337-1344.

JunG, H., AND KaRATO, S. I. 2001b. Water-induced fabric transitions in olivine. Science, 293(5534), 1460-1463.

Jung, H., Katayama, I., JiAng, Z., Hiraga, T., And Karato, S. 2006. Effect of water and stress on the lattice-preferred orientation of olivine. Tectonophysics, 421(1-2), 1-22.

KAMINSKI, E., AND RiBE, N. M. 2001. A kinematic model for recrystallization and texture development in olivine polycrystals. Earth and Planetary Science Letters, 189(3-4), 253-267.

Kaneshima, S., AND Silver, P. G. 1995. Anisotropic loci in the mantle beneath central Peru. Physics of The Earth and Planetary Interiors, 88(3-4), 257-272.

KARATO, S. 1992. On the Lehmann discontinuity. Geophysical Research Letters, 19(22), 22552258.

KARATO, S. 2003. Mapping water content in the upper mantle. In: Inside the subduction factory. Geophysical Monograph, vol. 138. American Geophysical Union.

KARATO, S.-I. 1988. The role of recrystallization in the preferred orientation of olivine. Physics of The Earth and Planetary Interiors, 51(1-3), 107-122. 
KARATO, S.-I., AND JUNG, H. 1998. Water, partial melting and the origin of the seismic low velocity and high attenuation zone in the upper mantle. Earth and Planetary Science Letters, 157(3-4), 193-207.

Karato, S.-I., AND WU, P. 1993. Rheology of the Upper Mantle: A Synthesis. Science, 260(5109), 771-778.

KARATO, S.-I., JunG, H., KATAYAMA, I., AND SKEMER, P. 2008. Geodynamic Significance of Seismic Anisotropy of the Upper Mantle: New Insights from Laboratory Studies. Annual Review of Earth and Planetary Sciences, 36(1), 59-95.

KARKI, B. B., STIXRUDE, L., AND WENTZCOVITCH, R. M. 2001. High-pressure elastic properties of major materials of Earth's mantle from first principles. Reviews of Geophysics, 39(4), 507-534.

KATAyAmA, I., KARATO, S. I., AND JUNG, H. 2004. New type of olivine fabric from deformation experiments at modest water content and low stress. Geology, 32(12), 1045-1048.

KATAYAMA, I., AND KARATO, S.-I. 2006. Effect of temperature on the B- to C-type olivine fabric transition and implication for flow pattern in subduction zones. Physics of The Earth and Planetary Interiors, 157(1-2), 33-45.

Katz, R. F., Spiegelman, M., And Holtzman, B. 2006. The dynamics of melt and shear localization in partially molten aggregates. Nature, 442(7103), 676-679.

Kelemen, P. B., AND DiCK, H. J. B. 1995. Focused melt flow and localized deformation in the upper mantle: Juxtaposition of replacive dunite and ductile shear zones in the Josephine peridotite, SW Oregon. Journal of Geophysical Research, 100(B1), 423-438.

Kelemen, P., Hirth, G., Shimizu, N., Spiegelman, M., AND DiCK, H. 1997. A review of melt migration processes in the asthenospheric mantle beneath oceanic spreading centers. Philosophical Transactions of the Royal Society of London, A355, 283-318.

Kelemen, P., Braun, M., And Hirth, G. 2000. Spatial distribution of melt conduits in the mantle beneath oceanic spreading ridges: Observations from the Ingalls and Oman ophiolites. G-cubed, 1, 1999GC000012.

Kelley, K. A., Plank, T., Grove, T. L., Stolper, E. M., Newman, S., And Hauri, E. 2006. Mantle melting as a function of water content beneath back-arc basins. Journal of Geophysical Research, 111(B09208, doi:10.1029/2005JB003732).

Kelly, K. R., WARD, R. W., Treitel, S., AND Alford, R. M. 1976. Synthetic seismograms: a finite-difference approach. Geophysics, 41(1), 2-27.

Kendall, J. M. 1994. Teleseismic arrivals at a mid-ocean ridge: effects of mantle melt and anisotropy. Geophysical Research Letters, 21(4), 301-304.

Kendall, J. M. 2000. Seismic anisotropy in the boundary layers of the mantle. Pages 149175 of: Karato, S., Forte, A. M., Liebermann, R., Masters, G., and Stixrude, L. (eds), Earth's deep interior: Mineral Physics and Tomography from atomic to the global scale. Geophysical Monograph Series, vol. 117. Washington, D.C.: AGU.

Kendall, J.-M., AND Silver, P. 1996. Constraints from seismic anisotropy on the nature of the lowermost mantle. Nature, 381(6581), 409-412.

Kendall, J. M., Stuart, G. W., Bastow, I. D., Ebinger, C. J., And Keir, D. 2005. Magma-assisted rifting in Ethiopia. Nature, 433(7022), 146-148. 
Kendall, J.-M., Pilidou, S., Keir, D., Bastow, I., Stuart, G., And Ayele, A. 2006. Mantle upwellings, melt migration and magma assisted rifting in Africa: Insights from seismic anisotropy. Pages 57-74 of: Yirgu, G., Ebinger, C. J., And Maguire, P. K. H. (eds), Structure and evolution of the rift systems within the Afar volcanic province, Northeast Africa, vol. 259. Geol. Soc. London.

Kennett, B. L. N., Engdahl, E. R., And Buland, R. 1995. Constraints on seismic velocities in the Earth from traveltimes. Geophysical Journal International, 122(1), 108-124.

KePPleR, H. 1996. Constraints from partitioning experiments on the composition of subduction-zone fluids. Nature, 380(6571), 237-240.

KERN, H. 1993. P- and S-wave anisotropy and shear-wave splitting at pressure and temperature in possible mantle rocks and their relation to the rock fabric. Physics of The Earth and Planetary Interiors, 78(3-4), 245-256.

KINCAID, C., AND GRIfFITHS, R. W. 2003. Laboratory models of the thermal evolution of the mantle during rollback subduction. Nature, 425, 58-62.

KINCAID, C., AND SACKS, I. 1997. Thermal and dynamical evolution of the upper mantle in subduction zones. Journal of Geophysical Research B: Solid Earth, 102(6), 12295-12315.

KING, P. R. 2000. Tectonic reconstructions of New Zealand: 40 Ma to the Present. New Zealand Journal of Geology and Geophysics, 43, 611-638.

KING, S. D. 2001. Subduction zones: observations and geodynamic models. Physics of The Earth and Planetary Interiors, 127(1-4), 9-24.

Klosko, E., Wu, F., Anderson, H., Eberhart-Phillips, D., McEvilly, T., Audoine, E., SAVAGE, M., AND GLEDHILL, K. 1999. Upper mantle anisotropy in the New Zealand region. Geophysical Research Letters, 26(10), 1497-1500.

Kneller, E. A., VAn KeKen, P. E., Karato, S.-I., And PARK, J. 2005. B-type olivine fabric in the mantle wedge: Insights from high-resolution non-Newtonian subduction zone models. Earth and Planetary Science Letters, 237(3-4), 781-797.

Kneller, E. A., van Keken, P., Katayama, I., And Karato, S. 2007. Stress, strain, and B-type olivine fabric in the fore-arc mantle: Sensitivity tests using high-resolution steady-state subduction zone models. Journal of Geophysical Research, 112(B04406, doi:10.1029/2006JB004544), 1-17.

KNeller, E. A., LONG, M. D., AND VAn KEKEN, P. E. 2008. Olivine fabric transitions and shear wave anisotropy in the Ryukyu subduction system. Earth and Planetary Science Letters, 268(3-4), 268-282.

Kohlstedt, D. L., AND Zimmerman, M. E. 1996. Rheology of partially molten mantle rocks. Annual Review of Earth and Planetary Sciences, 24, 41-62.

KumazAWA, M. 1969. The elastic constants of single-crystal orthopyroxene. Journal of Geophysical Research, 74, 5973-5980.

KumAZAWA, M., AND ANDERSON, O. 1969. Elastic properties, pressure derivates, and temperature derivates of single-crystal olivine and single-crystal forsterite. Journal of Geophysical Research, 74, 5961-5972. 
LAMARChE, G., BARNeS, P., AND BulL, J. 2006. Faulting and extension rate over the last 20,000 years in the offshore Whakatane Graben, New Zealand continental shelf. Tectonics, 25(4), 1-24.

LAMB, S. 2006. Shear stresses on megathrusts: Implications for mountain building behind subduction zones. Journal of Geophysical Research, 111(B07401, doi:10.1029/2005JB003916).

LASSAK, T. M., FOUCH, M. J., HAll, C. E., AND KAMINSKI, E. 2006. Seismic characterization of mantle flow in subduction systems: Can we resolve a hydrated mantle wedge? Earth and Planetary Science Letters, 243(3-4), 632-649.

LeVin, V., Lerner-LAM, A., AND Menke, W. 1995. Anomalous mantle structure at the Proterozoic-Paleozoic boundary in northeastern US. Geophysical Research Letters, 22(2), 121-124.

Levin, V., MenKe, W., AND PARK, J. 1999. Shear wave splitting in the Appalachians and the Urals: A case for multilayered anisotropy. Journal of Geophysical Research B: Solid Earth, 104(B8), 17975.

LEVIN, V., OKAYA, D., AND PARK, J. 2006. Shear wave birefringence in wedge-shaped anisotropic regions. Geophysical Journal International, 168, 275-286.

Li, L., RAterron, P., Weidner, D., AND Chen, J. 2003. Olivine flow mechanisms at 8 GPa. Physics of the Earth and Planetary Interiors, 138, 113-129.

LiTTLE, A., AND JONES, A. 1998. Seven million years of strike-slip and related off-fault deformation, northeastern Marlborough fault system, South Island, New Zealand. Tectonics, 17(2), 285-302.

LiU, E., Chapman, M., ZHANG, Z., AND QUeEN, J. 2006. Frequency-dependent anisotropy: Effects of multiple fracture sets on shear-wave polarizations. Wave Motion, 44(1), 44-57.

LiU, E., Maultzsch, S., Chapman, M., Li, X. Y., Queen, J. H., And Zhang, Z. 2003. Frequency-dependent seismic anisotropy and its implication for estimating fracture size in low porosity reservoirs. The Leading Edge, 22(7), 662-665.

LiU, J., LiU, Q.-Y., GuO, B., YUEN, D. A., AND SONG, H.-Z. 2007. Small-scale convection in the upper mantle beneath the Chinese Tian Shan Mountains. Physics of The Earth and Planetary Interiors, 163(1-4), 1-4.

LiU, K., ZHANG, Z., HU, J., AND TeNG, J. 2001. Frequency band-dependence of S-wave splitting in China mainland and its implications. Science in China, 44(7), 659-665.

LIU, Z., AND BIRD, P. 2006. Two-dimensional and three-dimensional finite element modelling of mantle processes beneath central South Island, New Zealand. Geophysical Journal International, 165(3), 1003-1028.

LONG, M. D., AND VAN DER HILST, R. D. 2006. Shear wave splitting from local events beneath the Ryukyu arc: Trench-parallel anisotropy in the mantle wedge. Physics of The Earth and Planetary Interiors, 155(3-4), 300-312.

LONG, M. D., DE HoOp, M. V., AND VAN DER Hilst, R. D. 2008. Wave-equation shear wave splitting tomography. Geophysical Journal International, 172(1), 311-330.

LYNN, H. 2004. The winds of change: Anisotropic rocks - Their preferred direction of fluid flow and their associated seismic signatures - Part 1. The Leading Edge, 23(11), 1156-1162. 
MAINPRICE, D. 1990. A fortan program to calculate seismic anisotropy from the lattice preferred orientation of minerals. Computers \& Geosciences, 16(3), 385-393.

MAINPRICE, D. 1997. Modelling the anisotropic seismic properties of partially molten rocks found at mid-ocean ridges. Tectonophysics, 279(1-4), 161-179.

MAINPRICE, D. 2007. Seismic anisotropy of the deep Earth from a mineral and rock physics perspective. Pages 437-491 of: GerAld Schubert, G. D. P. (ed), Treatise in Geophysics, vol. 2, Mineral Physics. Elsevier.

MAINPRICE, D., BARRUOL, G., AND Ben IsMAÏL, W. 2000. The seismic anisotropy of the Earth mantle: From single crystal to polycrystal. In: KARATO, S., FORTE, A. M., LIEBERMANN, R., MAsters, G., AND StixRUde, L. (eds), Earth's Deep Interior: Mineral Physics and Tomography from the Atomic to the Global Scale, vol. 117. AGU Geophysical Monograph.

MainPrice, D., Tommasi, A., Couvy, H., Cordier, P., And Frost, D. J. 2005. Pressure sensitivity of olivine slip systems and seismic anisotropy of Earth's upper mantle. Nature, 433(7027), 731-733.

MAINPRICE, D., AND NiCOlas, A. 1989. Development of shape and lattice preferred orientations: application to the seismic anisotropy of the lower crust. Journal of Structural Geology, 11(1-2), 175-189.

MAINPRICE, D., AND SILVER, P. G. 1993. Interpretation of SKS-waves using samples from the subcontinental lithosphere. Physics of The Earth and Planetary Interiors, 78(3-4), 257-280.

MANNING, C. 1996. Effect of sediment on aqeous silica transport in subduction zones. Pages 277-284 of: BeBOUt, G. (ed), Subduction: Top to Bottom. Geophysical Monograph Series, vol. 96. AGU, Washington D.C.

MARSON-PidgeOn, K., AND SAVAGE, M. 1997. Frequency-dependent anisotropy in Wellington, New Zealand. Geophysical Research Letters, 24, 3297-3300.

Marson-Pidgeon, K., Savage, M. K., Gledhill, K., And Stuart, G. 1999. Seismic anisotropy beneath the lower half of the North Island, New Zealand. Journal of Geophysical Research B: Solid Earth, 104(B9), 20277-20286.

Marson-PidgeOn, K. A., And SAVAGE, M. K. 2004. Modeling shear-wave splitting at Wellington, New Zealand. Geophysical Journal International, 157, 853-864.

Martynov, V. G., Vernon, F. L., Kilb, D. L., And Roecker, S. W. 2004. Directional variations in travel-time residuals of teleseismic $P$ waves in the crust and mantle beneath northern Tien Shan. Bulletin of the Seismological Society of America, 94(2), 650-664.

Masters, G., LAske, G., Bolton, H., And DziewOnski, A. M. 2000. The relative behavior of shear velocity, bulk sound speed, and compressional velocity in the mantle: Implications for chemical and thermal structure. In: KARATO, S. (ed), Earth's deep structure, vol. 117. AGU Geophysical Monograph.

Matcham, I., Savage, M. K., And Gledhill, K. R. 2000. Distribution of seismic anisotropy in the subduction zone beneath the Wellington region, New Zealand. Geophysical Journal International, 140(2000), 1-10.

Maultzsch, S., Chapman, M., LiU, E., AND Li, X. 2003. Modelling frequency-dependent seismic anisotropy in fluid-saturated rock with aligned fractures: implication of fracture size estimation from anisotropy measurements. Geophysical Prospecting, 51, 381-392. 
MAVKO, G. 1980. Velocity and Attenuation in Partially Molten Rocks. Journal of Geophysical Research, 85(B10), 5173-5189.

MAVKO, G., AND NUR, A. 1975. Melt squirt in the asthenosphere. Journal of Geophysical Research, 80(11), 1444-1448.

MAVKo, G., MuKerJI, T., AND DVORKIN, J. 1998. The rock physics handbook : tools for seismic analysis in porous media. Cambridge : Cambridge University Press.

MCKENZIE, D. 1979. Finite deformation during fluid flow. Geophysical Journal of the Royal astronomical Society, 58, 689-715.

Mehl, L., Hacker, B., Hirth, G., And Kelemen, P. 2003. Arc-parallel flow within the mantle wedge: Evidence from the accreted Talkeetna arc, south central Alaska. Journal of Geophysical Research B: Solid Earth, 108(8), 1-18.

MizuKami, T., WALLiS, S. R., AND YAmamoto, J. 2004. Natural examples of olivine lattice preferred orientation patterns with a flow-normal a-axis maximum. Nature, 427(6973), $432-436$.

Montagner, J. P. 2002. Upper mantle low anisotropy channels below the Pacific Plate. Earth and Planetary Science Letters, 202(2), 263-274.

MoONEy, H. M. 1970. Upper mantle inhomogeneity beneath New Zealand: seismic evidence. Journal of Geophysical Research, 75, 285 - 309.

MoOres, E. M., AND Twiss, R. J. 1995. Tectonics. W. H. Freeman and Company.

Morlet, J., Arens, G., Fourgeau, E., AND Glard, D. 1982. Wave propagation and sampling theory-Part I: Complex signal and scattering in multilayered media. Geophysics, 47(2), 203-221.

Morley, A. M., Stuart, G. W., Kendall, J. M., And Reyners, M. 2006. Mantle wedge anisotropy in the Hikurangi subduction zone, central North Island, New Zealand. Geophysical Research Letters, 33(5).

Mortimer, N., AND PARKINSON, D. 1996. Hikurangi Plateau: A Cretaceous large igneous province in the southwest Pacific Ocean. Journal of Geophysical Research B: Solid Earth, 101(1), 687-696.

Musgrave, M. J. P. 1970. Crystal acoustics. San Francisco Holden-Day Inc.

NAIRN, I., AND COLE, J. 1981. Basalt dikes in the 1886 Tarawera Rift. New Zealand Journal of Geology \& Geophysics, 24(5-6), 585-592.

NAKAJIMA, J., AND HaSegaWA, A. 2004. Shear-wave polarization anisotropy and the subduction-induced flow in the mantle wedge of northeastern Japan. Earth and Planetary Science Letters, 225, 365-377.

NAKAJIMA, J., TAKeI, Y., AND HASEGAWA, A. 2005. Quantitative analysis of the inclined low-velocity zone in the mantle wedge of northeastern Japan: A systematic change of melt-filled pore shapes with depth and its implications for melt migration. Earth and Planetary Science Letters, 234(1-2), 59-70.

Nicol, A., Mazengarb, C., Chanier, F., Rait, G., Uruski, C., And Wallace, L. 2007. Tectonic evolution of the active Hikurangi subduction margin, New Zealand, since the Oligocene. Tectonics, 26(TC4002, doi:10.1029/2006TC002090). 
Nicolas, A. 1984. Principles of Rock Deformation. D. Reidel, Norwell.

Nicolas, A., AND Christensen, N. 1987. Composition, Structure and Dynamics of the Lithosphere-Asthenosphere System. Vol. 16. American Geophysical Union, Washington, DC. Chap. Formation of anisotropy in upper mantle peridotites - a review, pages 111-123.

Nicolas, A., Boudier, F., AND Boullier, A. M. 1973. Mechanisms of flow in naturally and experimentally deformed peridotites. American Journal of Sciences, 273(10), 853-876.

Nishimura, C., AND FORSYTH, D. 1989. The anisotropic structure of the upper mantle in the Pacific. Geophysical Journal, 96(2), 203-229.

NYE, J. 1985. Physical properties of crystals: Their representation by tensors and matrices. Oxford University Press, New York.

ODA, H., AND SHIMIZU, H. 1997. S wave splitting observed in southwest Japan. Tectonophysics, 270(1-2), 73.

OF MONTPellier, U. Shear-wave splitting data base. http://www.gm.univmontp2.fr/splitting/DB/.

ÖZAlaybeY, S., AND CHEN, W.-P. 1999. Frequency-dependent analysis of SKS/SKKS waveforms observed in Australia: evidence for null birefringence. Physics of the Earth and Planetary Interiors, 114, 197-210.

PANDEY, O. 1981. Terrestrial heat flow in the North Island of New Zealand. Journal of Volcanology and Geothermal Research, 10, 309-316.

PANNING, M., AND ROMANOWICZ, B. 2006. A three-dimensional radially anisotropic model of shear velocity in the whole mantle. Geophysical Journal International, 167(1), 361-379.

PARK, J., AND LeVIN, V. 2002. Geophysics: Seismic anisotropy: Tracing plate dynamics in the mantle. Science, 296(5567), 485-489.

PEACOCK, S. 2003. Thermal structure and metamorphic evolution of subducting slabs. Pages 7-22 of: UNION, A. G. (ed), Inside the subduction factory. Geophysical Monograph, vol. 138. AGU.

PEACOCK, S. 1996. Thermal and petrological structure of subduction zones. Pages 119-133 of: BeBOUt, G. (ed), Subduction: From top to bottom, vol. 96. AGU Geophysical Monograph.

PEACOCK, S., AND WANG, K. 1999. Seismic consequences of warm versus cool subduction metamorphism: Examples from southwest and northeast Japan. Science, 286(5441), 937939.

Pearce, J. A., And Peate, D. W. 1995. Tectonic Implications of the Composition of Volcanic arc Magmas. Annual Review of Earth and Planetary Sciences, 23(1), 251-285.

Pera, E., Mainprice, D., AND Burlini, L. 2003. Anisotropic seismic properties of the upper mantle beneath the Torre Alfina area (Northern Apennines, Central Italy). Tectonophysics, 370(1-4), 11-30.

Peyton, V. 2001. Mantle flow at a slab edge; seismic anisotropy in the Kamchatka region. Geophysical Research Letters, 28, 379-382. 
Peyton, V., Levin, V., Park, J., Brandon, M., Lees, J., GordeeV, E., And Ozerov, A. 2001. Mantle flow at a slab edge: Seismic anisotropy in the Kamchatka region. Geophysical Research Letters, 28(2), 379-382.

Plomerová, J., Arvidsson, R., Babuška, V., Granet, M., KulhaneK, O., Poupinet, G., AND SILENY, J. 2001. An array study of lithospheric structure across the Protogine zone, Varmland, south-central Sweden - signs of a paleocontinental collision. Tectonophysics, 332(1-2), 1-21.

Plomerová, J., BabušKA, V., Vecsey, L., KozlovskayA, E., AND RAitA, T. 2006. Proterozoic-Archean boundary in the mantle lithosphere of eastern Fennoscandia as seen by seismic anisotropy. Journal of Geodynamics, 41(4), 400-410.

POLET, J. 2007. A map of relative P wave delay times across southern California. Geochemistry Geophysics Geosystems, 8, Q10003, doi:10.1029/2007GC001626.

Polet, J., Silver, P. G., Beck, S., WAllace, T., ZANDT, G., Ruppert, S., Kind, R., AND Rudloff, A. 2000. Shear wave anisotropy beneath the Andes from the BANJO, SEDA, and PISCO experiments. Journal of Geophysical Research B: Solid Earth, 105(3), 6287-6304.

Poli, S., AND SCHMidt, M. W. 2002. Petrology of subducted slabs. Annual Review of Earth and Planetary Sciences, 30(1), 207-235.

PRASAD, C., AND BOCK, G. 1987. P-wave travel-times from the Tonga subduction zone to stations on the oceanic side of the Tonga-Kermadec trench. Geophysical Research Letters, 14(1), 9-12.

Pulford, A., AND STERN, T. 2004. Pliocene exhumation and landscape evolution of central North Island: The role of the upper mantle. Journal of Geophysical Research, 109.

Rathore, J. S., Fjaer, E., Holt, R. M., And Renlie, L. 1995. P- and S-wave anisotropy of a synthetic sandstone with controlled crack geometry. Geophysical Prospecting, 43(6), 711-728.

RAWLINSON, N., AND KENNETT, B. 2004. Rapid estimation of relative and absolute delay times across a network by adaptive stacking. Geophysical Journal International, 157(1), 332-340.

Reilly, W., Whiteford, C., AND DoOne, A. 1977. North Island: Gravity map of New Zealand. 1:1000000 Bouguer and isostatic anomalies. 1st ed. Department of Science and Industrial Research, Wellington, New Zealand.

Restivo, A., AND HelfFrich, G. 2006. Core-mantle boundary structure investigated using SKS and SKKS polarization anomalies. Geophysical Journal International, 165(1), 288-302.

REuss, A. 1929. Berechnung der Fliessgrenze von Mischkristallen auf Grund der Plastizitätsbedingung für Einkristalle. Zeitschrift für angewandte Mathematik und Mechanik, 9, 49-58.

REYNers, M., AND StUART, G. 2002 (June 2002). The central North Island passive seismic experiment. Science report 2002/11. Institute of Geological and Nuclear Science.

REYNers, M., RoBinsOn, R., AND MCGINTY, P. 1997. Plate coupling in the northern South Island and southernmost North Island, New Zealand, as illuminated by earthquake focal mechanisms. Journal of Geophysical Research B: Solid Earth, 102(B7), 15197-15210. 
Reyners, M., Eberhart-Phillips, D., Stuart, G., And Nishimura, Y. 2006. Imaging subduction from the trench to $300 \mathrm{~km}$ depth beneath the central North Island, New Zealand, with Vp and Vp/Vs. Geophysical Journal International, 165(2), 565-583.

Reyners, M., Eberhart-Phillips, D., AND StuART, G. 2007. The role of fluids in lowercrustal earthquakes near continental rifts. Nature, 446, 1075-1078.

Ribe, N. M. 1989. Seismic anisotropy and mantle flow. Journal of Geophysical Research, 94, $4213-4223$.

RingwoOD, A. 1975. Composition and structure of the Earth's mantle. McGraw-Hill, New York.

Rioul, O., AND Vetterli, M. 1991. Wavelets and signal processing. Signal Processing Magazine, IEEE, 8(4), 14-38.

Ritzwoller, M., SHapiro, N., AND ZHONG, S. 2004. Cooling history of the Pacific lithosphere. Earth Planet. Sci. Letts., 226, 69-84.

Robertson, G., AND WoOdhouse, J. 1996. Ratio of relative S to P velocity heterogeneity in the Lower Mantle. Journal of Geophysical Research, 101, 21,795.

RoBinsON, R. 1976. Relative teleseismic travel-time residuals, North Island, New Zealand, and their relation to upper-mantle structure. Tectonophysics, 31, T41-T48.

Rowland, J. V., AND SibSON, R. H. 2001. Extensional fault kinematics within the Taupo Volcanic Zone, New Zealand: soft-linked segmentation of a continental rift system. New Zealand Journal of Geology \& Geophysics, 44, 271-283.

RÜMPKER, G., AND SILVER, P. G. 1998. Apparent shear-wave splitting parameters in the presence of verticallly varying anisotropy. Geophysical Journal International, 135, 790-800.

RÜMPKER, G., TOMMASI, A., AND KENDALL, J. M. 1999. Numerical simulations of depthdependent anisotropy and frequency-dependent wave propagation effects. Journal of Geophysical Research, 104(B10), 23141.

RÜMPKer, G., RYBerG, T., BOCK, G., AND GROUP, D. S. 2003. Boundary-layer mantle flow under the Dead Sea transform fault inferred from seismic anisotropy. Nature, 425, 497-501.

Russo, R. M., AND SILVER, P. G. 1994. Trench-parallel flow beneath the Nazca Plate from seismic anisotropy. Science, 263(5150), 1105-1111.

SAlmon, M. L., SAVAGE, M., AND STERN, T. 2005. Seismic attenuation, temperature, H2O, mantle melting and rock uplift, central North Island, New Zealand. Eos Trans. AGU, 86(52), T13B-0478.

Salmon, M. L., Savage, M., And Stern, T. A. 2006. Geophysical Evidence for Convectively Removed Mantle and Lower Crust Behind a Continental Back-arc. Eos Trans. AGU, Fall Meet. Suppl., Abstract, T31C-0462.

SANDVOL, E., AND NI, J. 1997. Deep azimuthal seismic anisotropy in the southern Kurile and Japan subduction zones. Journal of Geophysical Research B: Solid Earth, 102(B5), 99119922.

SANDVOL, E., NI, J., KIND, R., AND ZHAO, W. 1997. Seismic anisotropy beneath the southern Himalayas-Tibet collision zone. Journal of Geophysical Research B: Solid Earth, 102(B8), $17813-17823$. 
SAVAGE, M. 1999. Seismic anisotropy and mantle deformation: What have we learned from shear wave splitting? Reviews of Geophysics, 37(1), 65-106.

Savage, M., ANd Silver, P. G. 1993. Mantle deformation and tectonics: constraints from seismic anisotropy in western United States. Physics of the Earth and Planetary Interiors, 78, 207-227.

SAVAGe, M., AND Silver, P. 1994. How thick is the continental asthenosphere? In: Abstracts of IASPEI 27th General Assembly, Wellington, New Zealand, W3.16.

SCHMELING, H. 1985. Numerical models on the influence of partial melt on elastic, anelastic and electric properties of rocks. Part I: elasticity and anelasticity. Physics of The Earth and Planetary Interiors, 41(1), 34-57.

SChMid, C., VAN DER LeE, S., AND GiARDini, D. 2004. Delay times and shear wave splitting in the Mediterranean region. Geophysical Journal International, 159(1), 275-290.

Schulte-Pelkum, V., And Blackman, D. K. 2003. A synthesis of seismic P and S anisotropy. Geophysical Journal International, 154(1), 166-178.

Seward, A. M., Smith, E. C., And Henderson, M. 2006. Behind the subduction zone: Seismic wave speeds in the upper mantle wedge beneath the central North Island, New Zealand. In: American Geophysical Union, Fall Meeting 2006, Abstract T23C-0507.

Seward, A. M., E., S., AND Henderson, M. 2008. Models of the upper mantle beneath the central North Island from sub-horizontal P-waves (Pn). in preparation.

SilenY, J., AND PlOMEROVÁ, J. 1996. Inversion of shear-wave splitting parameters to retrieve three-dimensional orientation of anisotropy in continental lithosphere. Physics of the Earth and Planetary Interiors, 95(3-4), 277-292.

Silver, P. G. 1996. Seismic anisotropy beneath the continents: Probing the depths of geology. Annual Review of Earth and Planetary Sciences, 24, 385-432.

Silver, P. G., AND CHAN, W. 1988. Implications for continental structure and evolution from seismic anisotropy. Nature, 335(6185), 34-39.

Silver, P. G., AND SAVAGE, M. K. 1994. The interpretation of shear-wave splitting parameters in the presence of two anisotropic layers. Geophysical Journal International, 119(3), 949-963.

Silver, P. G., Mainprice, D., Ismaïl, W. B., Tommasi, A., And Barroul, G. 1999. Mantle structural geology from seismic anisotropy. In: FEI, Y., BERTKA, C. M., AND Mysen, B. O. (eds), Mantle Petrology: Field Observations and high Pressure experimentations: a Tribute to Francis R. (Joe) Boyd, vol. Special Publication No.6. The Geochemical Society.

Silver, P., AND CHAN, W. 1991. Shear wave splitting and subcontinental mantle deformation. Journal of Geophysical Research, 96(B10), 16,429-16,454.

SimONS, F., AND VAN DER HILST, R. 2003. Seismic and mechanical anisotropy and the past and present deformation of the Australian lithosphere. Earth and Planetary Science Letters, $211(3-4), 271-286$.

SMith, E. G. C., Stern, T., AND ReYNers, M. 1989. Subduction and back-arc activity at the Hikurangi convergent margin, New Zealand. Pure and Applied Geophysics, 129(1-2), 203-231. 
Smith, G. P., Wiens, D. A., Fischer, K. M., Dorman, L. M., Webb, S. C., AND HildeBRAND, J. A. 2001. A complex pattern of mantle flow in the Lau backarc. Science, 292(5517), 713-716.

Smith, I. E. M., AND PRICE, R. C. 2006. The Tonga-Kermadec arc and Havre-Lau back-arc system: Their role in the development of tectonic and magmatic models for the western Pacific. Journal of Volcanology and Geothermal Research, 156(3-4), 315-331.

Spiegelman, M., Kelemen, P. B., And Aharonov, E. 2001. Causes and cosequences of flow organization during melt transport: The reaction infiltration instability in compactible media. Journal of Geophysical Research, 106(B2), 2061-2077.

STEIN, S., AND WYSESSION, M. E. 2003. An introduction to seismology, earthquakes, and earth structure. Blackwell.

SterN, R. 2002. Subduction zones. Reviews of Geophysics, 40(1012), doi:10.1029/2001RG000108.

STERN, T. 1985. A back-arc basin formed within continental lithosphere: the Central Volcanic region of New Zealand. Tectonophysics, 112, 385-409.

Stern, T., Smith, E., Davey, F., And Muirhead, K. 1987. Crustal and upper mantle structure of the northwestern North Island, New Zealand, from seismic refraction data. Geophysical Journal of the Royal astronomical Society, 91, 913-936.

Stern, T. A., Stratford, W. R., And SAlmon, M. L. 2006. Subduction evolution and mantle dynamics at a continental margin: Central North Island, New Zealand. Reviews of Geophysics, 44(RG4002, doi:10.1029/2005RG000171), 1-36.

STERN, T. 1987. Asymmetric back-arc spreading, heat flux and structure associated with the Central Volcanic Region of New Zealand. Earth and Planetary Science Letters, 85, 265-276.

Stern, T., AND DAVEY, F. 1987. A seismic investigation of the crustal and upper mantle structure within the Central Volcanic Region of New Zealand. New Zealand Journal of Geology and Geophysics, 30, 217-231.

Stern, T., AND DAVEY, F. 1989. Crustal structure and origin of basins formed behind the Hikurangi subduction zone, New Zealand. Pages 73-85 of: PRICE, R. (ed), Origin and Development of Sedimentary Basins and their Energy Sources. Geophysical Monograph Series, American Geophysical Union.

Stratford, W. R., AND Stern, T. A. 2004. Strong seismic reflections and melts in the mantle of a continental back-arc basin. Geophysical Research Letters, 31(6), 1-5.

StRATford, W. R., AND STERN, T. A. 2006. Crust and upper mantle structure of a continental backarc: central North Island, New Zealand. Geophysical Journal International, 166, 469-484.

TAKEI, Y. 2001. Stress-induced anisotropy of partially molten media inferred from experimental deformation of a simple binary system under acoustic monitoring. Journal of Geophysical Research B: Solid Earth, 106(B1), 567-588.

TAKEI, Y. 2002. Effect of pore geometry on VP/VS: From equilibrium geometry to crack. Journal of Geophysical Research B: Solid Earth, 107(2), 6-1-6-12. 
TAKEI, Y. 2005. Deformation-induced grain boundary wetting and its effects on the acoustic and rheological properties of partially molten rock analogue. Journal of Geophysical Research B: Solid Earth, 110(12), 1-24.

TANDON, G., AND WENG, G. 1983. Effect of aspect ratio of inclusions on the elastic properties of unidirectionally aligned composites. Polymer Composites, 5(4), 327-333.

TAPley, W., TUll, J., Miner, L., AND Goldstain, P. 1990. SAC Command reference manual. University of California.

TATsumi, Y., AND EGGINS, S. 1995. Subduction zone magmatism. Blackwell, Malden, Mass.

Teanby, N., Kendall, J. M., And van Der BaAn, M. 2004. Automation of shear-wave splitting measurements using cluster analysis. Bulletin of the Seismological Society of America, 94(2), 453-463.

Thomsen, L. 1986. Weak elastic anisotropy. Geophysics, 51(10), 1954-1966.

Thomsen, L. 1995. Elastic anisotropy due to aligned cracks in porous rock. Geophysical Prospecting, 43(6), 805-829.

Tilmann, F. J., Benz, H. M., Priestley, K. F., And OKubo, P. G. 2001. P-wave velocity structure of the uppermost mantle beneath Hawaii from traveltime tomography. Geophysical Journal International, 146(3), 594-606.

TOD, S., AND LIU, E. 2002. Frequency-dependent anisotropy due to fluid flow in bed limited cracks. Geophysical Research Letters, 29(15), 1-4.

Tommasi, A., Mainprice, D., CAnOva, G., And Chastel, Y. 2000. Viscoplastic selfconsistent and equilibrium-based modeling of olivine lattice preferred orientations: Implications for the upper mantle seismic anisotropy. Journal of Geophysical Research B: Solid Earth, 105(B4), 7893-7908.

TOMMASI, A. 1998. Forward modeling of the development of seismic anisotropy in the upper mantle. Earth and Planetary Science Letters, 160(1-2), 1-13.

Tommasi, A., Vauchez, A., Godard, M., AND Belley, F. 2006. Deformation and melt transport in a highly depleted peridotite massif from the Canadian Cordillera: Implications to seismic anisotropy above subduction zones. Earth and Planetary Science Letters, 252(3-4), 245-259.

TORIUMI, M. 1984. Preferred orientation of olivine in mantle-derived peridotites and stress in the lithosphere. Journal of Physics of the Earth, 32, 259-271.

Torrence, C., And COMPO, G. P. 1998. A practical guide to wavelet analysis. Bulletin of the American Meterological Society, 79, 61-78.

Turcotte, D., And Schubert, G. 2002. Geodynamics. Cambridge University Press.

Twiss, R., AND Moores, E. M. 1997. Structural geology. W. H. Freeman and Company, New York.

Ulmer, P. 2001. Partial melting in the mantle wedge - The role of $\mathrm{H} 2 \mathrm{O}$ in the genesis of mantle-derived 'arc-related' magmas. Physics of the Earth and Planetary Interiors, 127(1-4), 215-232.

Ulmer, P., AND TrommsdorfF, V. 1995. Serpentine stability to mantle depths and subduction-related magmatism. Science, 268(5212), 858-861. 
UYEDA, S. 1982. Subduction zones: an introduction to comparative subductologys. Tectonophysics, 81(3-4), 133-159.

VAN KEKEN, P. E. 2003. The structure and dynamics of the mantle wedge. Earth and Planetary Science Letters, 215, 323-338.

VANDECAR, J., AND CROSSON, R. 1990. Determination of teleseismic relative phase arrival times using multi-channel cross-correlation and least squares. Bulletin of the Seismological Society of America, 80(1), 150-169.

Vauchez, A., Maumus, J., TOMmasi, A., And Barruol, G. 2000. Upper mantle deformation and seismic anisotropy in continental rifts. Physics and Chemistry of the Earth, Part A: Solid Earth and Geodesy, 25(2), 111-117.

Vauchez, A., Dineur, F., AND Rudnick, R. 2005. Microstructure, texture and seismic anisotropy of the lithospheric mantle above a mantle plume: Insights from the Labait volcano xenoliths (Tanzania). Earth and Planetary Science Letters, 232(3-4), 295-314.

Vecsey, L., PlomerovÁ, J., Kozlovskaya, E., AND BABUŠKA, V. 2007. Shear wave splitting as a diagnostic of variable anisotropic structure of the upper mantle beneath central Fennoscandia. Tectonophysics, 438(1-4), 57-77.

VERMA, R. 1960. Elasticity of some high-density crystals. Journal of Geophysical Research, 65(2), 757-766.

Villamor, P., AND BERRYMAN, K. 2001. A late quaternary extension rate in the Taupo Volcanic Zone, New Zealand, derived from fault slip data. New Zealand Journal of Geology and Geophysics, 44(2), 243-269.

Vinnik, L. P., Makeyeva, L. I., MileV, A., And Usenko, A. Y. 1992. Global patterns of azimuthal anisotropy and deformations in the continental mantle. Geophysical Journal International, 111, 433-447.

VOIGT, W. 1928. Lehrbuch der Kristallphysik. Teubner-Verlag, Leipzig.

Volti, T., Gorbatov, A., Shiobara, H., Sugioka, H., Mochizuki, K., And Kaneda, Y. 2006. Shear-wave splitting in the Mariana trough-a relation between back-arc spreading and mantle flow? Earth and Planetary Science Letters, 244(3-4), 566-575.

WAlcotT, R. 1984. Reconstructions of the New Zealand region for the Neogene. Palaeogeography, Palaeoclimatology, Palaeoecology, 46(1-3), 217-231.

WALCOTT, R. 1987. Geodetic strain and the deformational history of the North Island of New Zealand during the late Cainozoic. Philosophical Transactions of the Royal Society of London, A 321, 163-181.

WALcotT, R. 1998. Modes of oblique compression: Late Cenozoic tectonics of the South Island of New Zealand. Reviews of Geophysics, 36(1), 1-26.

WAllace, L. M., BeAVAN, J., MCCAFFrey, R., AND DARby, D. 2004. Subduction zone coupling and tectonic block rotations in the North Island, New Zealand. Journal of Geophysical Research B: Solid Earth, 109(12), 1-21.

WANG, J., Sinogeikin, S. V., InOue, T., AND BASS, J. D. 2003. Elastic properties of hydrous ringwoodite. American Mineralogist, 88(10), 1608-1611. 
WebB, T., AND ANDERSON, H. 1998. Focal mechanisms of large earthquakes in the North Island of New Zealand: slip partitioning at an oblique active margin. Geophysical Journal International, 134(1), 40-86.

WERNER, U., AND SHAPIRO, S. 1999. Frequency-dependent shear-wave splitting in thinly layered media with intrinsic anisotropy. Geophysics, 64(2), 604-608.

WESSEL, P., AND SMITH, W. H. F. 1995. New version of the generic mapping tools released. Eos Trans. AGU, 76, 329.

WiENS, D., AND SMith, G. 2003. Seismological constraints on structure and flow patterns within the mantle wedge. Pages 59-81 of: EILER, J. (ed), Inside the subduction factory. Geophysical Monograph, vol. 138. AGU.

Wiens, D. A., Kelley, K. A., AND PlanK, T. 2006. Mantle temperature variations beneath back-arc spreading centers inferred from seismology, petrology, and bathymetry. Earth and Planetary Science Letters, 248(1-2), 16-27.

Wiens, D. A., Conder, J. A., AND Faul, U. H. 2008. The Seismic Structure and Dynamics of the Mantle Wedge. Annual Review of Earth and Planetary Sciences, 36(1), 421-455.

Wilson, C. J. N., Houghton, B. F., McWilliams, M. O., Lanphere, M. A., Weaver, S. D., AND BRIGGS, R. M. 1995. Volcanic and structural evolution of Taupo Volcanic Zone, New Zealand: a review. Journal of Volcanology and Geothermal Research, 68(1-3), 1-28.

WinTERSTEIN, D. 1990. Velocity anisotropy terminology for geophysicists. Geophysics, 55(8), 1070-1088.

WinTERSTEIN, D. 1992. How shear-wave properties relate to rock fractures; simple cases. The Leading Edge, 11(9), 21-28.

WRIGHT, I., AND WALCOTT, R. 1986. Large tectonic rotation of part of New Zealand in the last 5 Ma. Earth E Planetary Science Letters, 80(3-4), 348-352.

WÜstefeld, A., And BoKelmann, G. 2007. Null Detection in Shear-Wave Splitting Measurements. Bulletin of the Seismological Society of America, 97(4), 1204-1211.

WÜstefeld, A., Bokelmann, G., Zaroli, C., AND BARRuol, G. 2008. SplitLab: A shearwave splitting environment in Matlab. Computers E Geosciences, 34(5), 515-528.

WyLLIE, P. J. 1992. Experimental petrology: Earth materials science. Chap. 4, pages 65-87 of: G. BROWN, C. H., AND Wilson, C. (eds), Understanding the Earth. Cambridge University Press.

ZHANG, S., AND KARATO, S.-I. 1995. Lattice preferred orientation of olivine aggregates deformed in simple shear. Nature, 375(6534), 774-777.

Zimmerman, M. E., Zhang, S., Kohlstedt, D. L., AND KARATO, S. I. 1999. Melt distribution in mantle rocks deformed in shear. Geophysical Research Letters, 26(10), 1505-1508. 